\title{
Abstracts of the 7th International Symposium on Pediatric Pain
}

\author{
June 25-29, 2006, \\ Vancouver, British Columbia
}

\section{PLENARY 1 - PAIN IN CHILD HEALTH}

\subsection{1}

\section{PAIN IN CHILDHOOD HIV/AIDS: THE CHALLENGES}

\section{R Albertyn}

Department of Paediatric Surgery, University of Cape Town, Cape Town, South Africa

Little is published on pain and related comfort issues in infants born HIV+, in contrast to the large body of literature available on the presence of pain in HIV+ adults. Infant HIV is perceived to be predominantly a third world problem, posing a unique set of challenges and management problems. The exact number of HIV+ children/infants in Sub Saharan Africa is not known. An estimated 25.4 million people in this region were HIV+ (2004), with $60 \%$ of child deaths in Africa attributed to HIV/AIDS.

The pain management and assessment of infant HIV is challenging due to: a lack of knowledge and specific training, lack of resources and staff shortages, and pain management not being seen as a treatment priority. Pain assessment is complicated by, amongst others, the lack of a specific method to measure pain and treatment efficacy as HIV+ infants respond to pain differently when compared to other sick infants.

Retrospective data analysis on 128 infant HIV deaths at the Red Cross Hospital during 2002-2004 showed that pain and discomfort are related to respiratory related infections, sepsis and failure to thrive in addition to gastrointestinal and neurological infections. Pain management was found to be inadequate due to the inability to measure pain, fear of opioids and lack of knowledge. This led to the development of the Paediatric HIV (PEDHIV) Scale and the Touch Visual Pain (TVP) Scale.

The TVP Scale was developed to measure pain and treatment efficacy in HIV+ infants. This scale includes a physical examination of the infant as well as observations. In addition, the PEDHIV Scale was developed to document the development of the disease in the infant.

Collaboration and the exchange of knowledge and information between first and third world health practitioners is vital for the future management of pain and discomfort associated with infant HIV/AIDS.

\subsection{2}

\section{DEVELOPMENTAL PERSPECTIVES ON CHRONIC ABDOMINAL PAIN \\ $\underline{\text { L Walker }}$}

Vanderbilt University Medical Center, Nashville, Tennessee, USA

This presentation integrates developmental and biopsychosocial perspectives in a conceptual model of the etiology, course, and outcomes of pediatric chronic abdominal pain (CAP). The utility of the model will be illustrated with examples from experimental studies in which we induced visceral discomfort in the laboratory in CAP patients and well controls using a symptom provocation test that we have developed and validated. According to our conceptual model, biopsychosoical risk factors may interact with precipitating events to upset the homeostasis of the brain-gut axis, resulting in acute abdominal pain that is a potential stressor for the child.
The impact of this pain stressor on children's daily functioning is shaped by children's appraisal and coping with the pain. Children who appraise their pain as a challenge are likely to respond with determination and accommodative coping strategies that maintain their daily lives with minimal interruption. This style of coping allows children to continue to achieve developmental milestones and thereby acquire resources that protect them in future acute episodes of pain and other stressors. These children are rarely seen for CAP in tertiary care centers.

In contrast, children who appraise their pain as a serious, uncontrollable threat tend to respond with fear and passive avoidant coping strategies in an effort to escape pain. This style of coping interferes with children's abilities to engage in activities necessary to achieve normal developmental milestones (eg, social and academic competence) and ultimately causes them to fall further behind their peers in their ability to handle both pain and other stressors. In this maladaptive trajectory, acute pain episodes evolve into a chronic pain syndrome that influences all aspects of the youth's functioning, creates further stress, and is associated with high levels of health service utilization.

Children's social ecology also plays an important role in their response to pain. Family members, peers, teachers, and health care providers may respond to children's pain behavior in ways that foster threat appraisals and passive coping associated with a maladaptive trajectory or, in contrast, foster challenge appraisals and accommodative coping associated with an adaptive trajectory.

We recently tested components of this model in a laboratory setting. Our results demonstrate how family stress, child competence, and parent attention to pain complaints interact to influence children's vulnerability to visceral discomfort.

\subsection{3}

PAIN MANAGEMENT IN PEDIATRIC PALLIATIVE CARE WITH SPECIAL EMPHASIS ON CANCER

\section{G Ljungman}

Pediatric Hematology/Oncology, Children's University Hospital, Uppsala, Sweden

Pain is the symptom most feared by young children with cancer (Lansky et al, 1989; Enskär et al, 1997). Despite significant advances in pain management over the recent decades, numerous studies have shown that pain is still common in pediatric oncology (Miser et al, 1987a; Miser et al, 1987b; McGrath et al, 1990a; Elliott et al, 1991; Ljungman et al, 1996; Ljungman et al, 1999; Collins et al, 2000; Collins et al, 2002). Pain is the second most prevalent symptom (49.1\%) next to lack of energy (49.7\%), and it is the second most distressing symptom (39.1\%) next to feeling sad (39.5\%) (Collins et al, 2000).

Children with cancer experience pain as a result of treatment (eg, sideeffects of chemotherapy), procedures, the cancer itself, and other etiologies. Treatment- and procedure-related pain dominate in the pediatric population (Miser et al, 1987a; Miser et al, 1987b; McGrath et al, 1990a; Elliott et al, 1991; Ljungman et al, 1996; Ljungman et al, 1999; Collins et al, 2000; Collins et al, 2002), while a preponderance of tumourrelated pain is found among adult patients (Coyle et al, 1990; Rawal et al, 
1993; Zech et al, 1995). This difference may partly be ascribed to the aggressive, multimodality therapy regimens generally used in pediatric oncology. Furthermore, childhood malignancies show a high initial rate of response to treatment, resulting in a rapid reduction and often disappearance, of tumour-related pain. Finally, children with unresponsive or relapsing, refractory cancer often deteriorate rapidly (Miser et al, 1987a). The major treatment-related problems are mucositis, neuropathic pain from the legs and abdomen, and inflammatory pain caused by infections. The main procedure-related problems are lumbar punctures, bone marrow aspirations and biopsies, port starts, iv sampling and cannulation, and finger sticks. There are still many obstacles to an acceptable pain situation for children with cancer.

\section{WORKSHOP 1A - ANALGESIC DRUGS: EFFICACY AND SAFETY IN INFANTS AND CHILDREN? \\ Chair: Tim Oberlander}

\subsection{1 \\ ANALGESIC DRUGS: EFFICACY AND SAFETY IN INFANTS AND CHILDREN \\ $\underline{K I S ~ A n a n d ~}^{1}$; IV Aranda ${ }^{2}$; R Carbajal $^{3}$ \\ ${ }^{1}$ Pediatrics, Anesthesiology, Pharmacology \& Neurobiology, UAMS \\ College of Medicine; Pain Neurobiology Laboratory, Arkansas \\ Children's Hospital Research Institute, Little Rock, Arkansas; \\ 2Pediatrics, Pharmacology, Pharmaceutical Sciences, Wayne State Univeristy School of Medicine, Children's Hospital of Michigan, Detroit, Michigan, USA; ${ }^{3}$ National Center of Resources to Fight Pain, Armand Trousseau Children's Hospital, Assistance Publique- Hôpitaux de Paris, Paris, France}

BACKGROUND: Practical difficulties and lack of a gold standard for pain assessment in infants and children have led to limited data on analgesic efficacy, whereas very large prospective studies and/or postmarketing surveys are needed for defining analgesic safety in this population.

PURPOSE: The workshop will explore the gaps in our knowledge regarding the efficacy and safety of commonly used analgesics in newborns, infants, and children. Preliminary strategies for closing these gaps will be outlined through active audience participation.

METHODS: Clinical studies examining therapeutic options for pain management for procedural, postoperative, recurrent or chronic pain will be identified by searching the PubMed (1980-2006) database, the Cochrane Controlled Trials Register, and personal files. The English language literature will be reviewed, excluding case reports and small case series.

RESULTS: Speakers and workshop participants will identify research priorities for examining the efficacy and safety of analgesic drugs in infants and children. Relative merits of various therapies will be discussed and evaluated, based on the strength of the evidence reported. Special populations will be defined that may have greater vulnerability to analgesic side effects or lack of efficacy. Novel outcomes or biomarkers for analgesic efficacy or safety will be identified. Interaction with nonpharmacological or complementary approaches will be explored. Obstacles for research or translation into clinical practice will be characterized and preliminary solutions proposed.

CONCLUSIONS: This workshop will prioritize the most important areas requiring research into the safety and efficacy of analgesic drugs in infants and children. Recommendations from this workshop will be submitted to research funding agencies and regulatory bodies in the United States, Canada, and other countries.

\section{WORKSHOP 1B - INTERNATIONAL ISSUES \\ IN CHILDREN'S PAIN: CHALLENGES IN THREE COUNTRIES \\ Chair: Allen Finley}

\subsection{1 \\ PREVALENCE OF PAIN AND BARRIERS TO CARE IN MEXICO AND LATIN AMERICA}

\section{MA Flores Muñoz}

Department of Pediatrics and Pediatric Pain Clinic, Mexico Géneral Hospital, México City, México

In Latin America, demographic indicators show that the percentage of the population aged $5-14$ years in 2000 was $21 \%$ compared with $12.5 \%$ in Europe. Bacterial infections parasites, viruses, cancer, chronic diseases and violence affect childhood. In the big cities the children of the street (niños de la calle) are born and live in suffering and pain. The prevalence of recurrent pain in Mexican school children is $24 \%$, of these, $70 \%$ persist with pain. Poverty, hunger, and political conditions interfere with the spread of medical knowledge and access to care.

Despite improvements in the detection and management of pain around the world there is a lack of knowledge about pediatric pain among pediatricians in Latin America. Mexican pediatricians were surveyed on pediatric pain during 1993 and this was repeated in 2004 with Latin American pediatricians from Ecuador, Argentina, Venezuela, República Dominicana, Colombia and Brazil. There were no significant differences. In 1993, 40\% of pediatricians disagreed with the use of opioids in children. In 2004, $42.5 \%$ disagreed. In 1993, $80 \%$ did not know a method of pain measurement and assessment in children; in 2004, 42.5\% still do not know.

\subsection{2}

\section{CHALLENGES AND OPPORTUNITIES OF PEDIATRIC PAIN IN THAILAND}

\section{W Krisanaprakornkit}

Department of Anesthesiology \& Clinical Epidemiology Unit, Faculty of Medicine, Khon Kaen University, Khon Kaen, Thailand

The reasons for undertreatment of pain in children of Thailand are mostly similar to others, but some specific issues create different challenges to those in Western countries, such as the difference in ethnicity and culture, and the lack of drugs, equipment, and knowledge of the health care team. In Southeast Asia, perception and meaning of pain may be different from western countries. Assessment of pain should be appropriate to lifestyle and culture, and pain treatment must be individualized for the particular situation.

In Thailand, there have been attempts to improve pain management in pediatric patients despite the limited resources. Many pain assessment tools, both behavioural and self-report measures have been validated in Thai children and are routinely used in some settings. These include NIPP for newborn, CHEOPS, FLACC, and Objective pain scales for preschool children and VAS, NRS, color and face scale for school age children. Many hospitals have developed Acute Pain Services that provide adult and pediatric care. Management systems suitable for each hospital have been set up to support patient care, including preprinted analgesic orders, regular monitoring, and effective consulting systems. Nonpharmacological pain management, such as relaxation, imagination and biofeedback, are now introduced as complementary therapies.

Thailand as a developing country has limited access to analgesic drugs as well as to pain specialists for pediatric patients. Staff education, training, support, and research must be emphasized to improve patient care and break down barriers to effective pain management. Right now, palliative care is included in our medical curriculum. Hopefully, pediatric pain would be more recognized and pediatric pain management in Thailand would be better. 
2.202.3

\section{PEDIATRIC PAIN IN INDIA: ISSUES AND CHALLENGES}

\section{DK Pawar}

Department of Anaesthesiology, All India Institute of Medical

Sciences, New Delhi, India

The health care system in our country is not child friendly in spite of the fact that $35 \%$ of our population comes from the pediatric age group. There are very few children's hospitals. There is a gross discrepancy in the standard of care available around the country. The metropolis might have five star facilities but the facilities are inadequate in small towns and rural areas where $80 \%$ of the population lives. When health care in general is in such a state, pediatric pain is a grossly neglected area.

Why it is so?

Lack of resources due to Different Priorities: In a developing nation priorities are given to infrastructure development like building of roads, ports, industry, generating electricity. Very little money is left for health care, where priorities are given to communicable and preventable disease, decreasing the infant and maternal mortality, eradicating diseases like tuberculosis and leprosy, and immunization. So the financial resources available for specialized care are limited.

Lack of awareness: Even the caregivers are not convinced of the hazards of not providing perioperative pain relief.

Fear of respiratory depression and addiction leads to avoiding narcotics even in severely painful conditions.

Lack of trained \& skilled manpower \& lack of infrastructure.

Nonavailability of drugs: India produces $80 \%$ of morphine used by the pharmaceutical industry all over the world, but it is not freely available. Physicians and pharmacists are not keen to take the trouble of keeping a cheap drug like morphine, as they have to take a license and keep an account.

What could be done?

Creating awareness among the surgeons, nurses and anaesthetists! Making people understand the benefits of providing pain relief. Helping them overcome the fear of narcotics. Helping them to understand the optimal utilization of locally available drugs and equipment. Helping them learn the skills to provide regional blocks, which provide excellent intraoperative and postoperative pain relief.

For the last 15 years, a few of us are trying to achieve these goals by conducting regular workshops, updates, and CMEs including live demonstration of skills to do blocks. So far we have covered almost all the states in northern, eastern and western India and in a small way helped to improve the situation.

\begin{tabular}{c}
\hline WORKSHOP 1C - MEASUREMENT OF PAIN \\
AS AN OUTCOME IN CLINICAL TRIALS: THE \\
PED-IMMPACT RECOMMENDATIONS \\
Chair: Patrick McGrath
\end{tabular}

\subsection{3}

MEASUREMENT OF PAIN AS AN OUTCOME IN CLINICAL TRIALS: THE PED-IMMPACT RECOMMENDATIONS

P McGrath ${ }^{1,5}$, L Stinson ${ }^{2,4}$, C von Baeyer ${ }^{3}$

1Departments of Psychology, Pediatrics and Psychiatry, Dalhousie University, Halifax, Nova Scotia; ${ }^{2}$ Faculty of Nursing, University of Toronto, Ontario; ${ }^{3}$ Departments of Psychology and Pediatrics, University of Saskatchewan, Saskatoon, Saskatchewan; ${ }^{4}$ Hospital for Sick Children, Toronto, Ontario; ${ }^{5}$ IWK Health Centre, Halifax, Nova Scotia

The mission of the Initiative on Methods, Measurement and Pain Assessment in Clinical Trials (IMMPACT) is to develop consensus recommendations for improving the design, execution and interpretation of clinical trials of treatments for pain. The Ped-IMMPACT project, to be summarized in this workshop, assembled experts in 2005 for a consensus conference on outcome measures related to pain for pediatric clinical trials for children and adolescents aged 3 to 18 years. Two technical reports were

subsequently commissioned, one covering self-report measures and the other covering observational or behavioral measures. Each report evaluated existing measures of pain in light of published evidence of reliability and validity, acceptability, responsiveness to events that increase or decrease pain, burden on the clinician, and other features. The review of self-report discussed the process of estimating one's own pain in relation to cognitive development, as well as the issue of distinguishing pain intensity from affective and evaluative aspects of pain. The review of observational measures addressed such issues as identification and weighing of reliable behavioral indices for different types of pain (procedural, post-operative, disease-related, recurrent). Both reports concluded with recommendations as to the pain measurement tools that are best supported by currently available evidence. The review process and the recommendations for specific tools will be outlined. The Ped-IMMPACT process is expected to contribute to the consistency and quality of future pediatric clinical trials including pharmacological and behavioral interventions for pain.

\subsection{3 \\ SYSTEMATIC REVIEW OF SELF-REPORT PAIN INTENSITY MEASURES FOR USE IN CLINICAL TRIALS IN CHILDREN AND ADOLESCENTS}

\section{IStinson}

\section{The Hospital for Sick Children, Toronto, Ontario}

A systematic review of the psychometric properties, interpretability and feasibility of self-report pain intensity measures for children and adolescents for use in clinical trials evaluating pain treatments was commissioned by the Ped-IMMPACT working group. Databases were searched for self-report measures of single-item ratings of pain intensity for children ages 3 to 18 years of age. A total of 34 single-item self-report measures were found. The measures' psychometric properties, interpretability and feasibility were evaluated independently by two investigators according to a set of psychometric criteria. Six single-item measures met the a priori criteria and were included in the final analysis. While these six scales were determined as psychometrically sound and show evidence of responsivity; they had varying degrees of interpretability and feasibility. No single scale was found to be optimal for use with all types of pain or across the developmental age span. Specific recommendations regarding the most psychometrically sound and feasible measures based on age/developmental level and type of pain will be discussed. Future avenues for research to strengthen the measurement of pain in clinical trials with children will be outlined.

\section{WORKSHOP 1D - COMMUNICATIVE DIMENSIONS OF PAIN IN CHILDREN: EXPRESSIVE AND RECEPTIVE FEATURES Chair: Kenneth Craig}

\subsection{1 \\ CAREGIVER JUDGEMENTS OF PAIN IN INFANTS THROUGHOUT THE FIRST YEAR OF LIFE \\ R Nader}

The University of British Columbia, Vancouver, British Columbia

For over two decades, researchers have studied the expression of pain in young infants to unlock the nature of this powerful experience early in life, with these studies resulting in the discrediting of numerous myths about infant pain (eg, infants are insensitive to pain). This work has great relevance for caregivers of preverbal infants who often are asked to assess the presence or absence and severity of pain an infant may be experiencing. This can be a challenging task, as they must extract information specific to pain from apparent generalized distress reactions, substantial variability in response among children, and similarities in response to noxious and nonnoxious aversive events, among other influences on their judgments. The presentation will illustrate how parent perceptions and assessment of pain change with the development of the infant and explore the relationship between parental assessments and behavioural indices of infant pain. The sociocommunications model of pain will be reviewed and used as a 
framework to present data from a study examining caregiver perceptions and assessments of pain in 160 infants in the first year of life. Data exploring the relationship between parental assessments and behavioural indices of infant pain will also be presented. Discussion will include an examination of various behavioural modalities infants use in the first year of life to express pain including facial activity, body movement and cry. Additionally, there will be discussions about the cues rated most important by caregivers when making judgments about pain in infants and how those subjective ratings of cue importance relate to the degree with which behavioural displays of infants actually predict caregiver ratings of pain.

\subsection{2 INTERPERSONAL DIMENSIONS OF PAIN CATASTROPHIZING IN CHILDREN}

\section{T Vervoort}

\section{Ghent University, Ghent, Belgium}

Dr Tine Vervoort will discuss the role of specific child factors affecting pain expression in the child, parental judgement of their child's pain and parental behaviour. In particular, specific attention will be paid to the communicative function of pain catastrophizing in children. This speaker will present data from both questionnaire studies and experimental studies with parents of both healthy school children and children who are suffering from pain. Discussion of the results will focus on the influence of pain catastrophizing and related child perceptions on the verbal and nonverbal expression of pain in children and on the effect child pain catastrophizing and related pain expressions have upon parental sensitivity and associated (behavioural) responses for their child's pain.

\subsection{3}

\section{PARENTAL SENSITIVITY TO PAIN CUES ACROSS}

\section{CHILDHOOD}

R Pillai Riddell ${ }^{1}$, R Nader ${ }^{2}$, T Vervoort ${ }^{3}$, K Craig $^{2}$

${ }^{1}$ York University/The Hospital for Sick Children, Toronto, Ontario; ${ }^{2}$ Unversity of British Columbia, Vancouver, British Columbia;

\section{${ }^{3}$ Ghent University, Belgium}

BACKGROUND: Because of the biological connectedness and commitments that parental guardianship engenders, parents play a unique role in the caregiving of the child in pain. Parents most often approach pediatric pain assessment with little to no formal medical training, relying heavily on personal, familial and cultural experiences. Given the nature of the parentchild relationship and the amount of time a parent spends with their own child, it would be expected that parents would have an in-depth understanding of the cues that their child would use to communicate pain. However, it has been suggested that parental sensitivity to their child's pain is strongly related to factors aside from overt child signalling such as parental beliefs about pain, parental stressors and parental psychopathology.

PURPOSE/METHODS: The purpose of this presentation is to review current research exploring parental sensitivity to pain cues from infancy to adolescence. Learning about potential sources of insensitivity within child-caregiver interaction is crucial to understanding how different factors lead to the mismanagement of pediatric pain.

Factors that influence parents' decisions about their child's pain levels will be discussed across the different age groups. To highlight current work focused on maternal sensitivity to her child's pain cues, new research contrasting the ability of infant variables (eg, global measures of infant facial response, body movement and cry) and maternal variables (eg, current psychological functioning, acculturative stress, perceived social support) to predict maternal pain judgments will be presented. This predictive model of subjective pain judgment will then be contrasted against a model predicting infant pain reactivity using a well-regarded, objective measure of infant pain (Neonatal Facial Coding System).

\section{WORKSHOP 1E - MORE THAN A BAD NIGHT'S SLEEP: CONNECTIONS BETWEEN PAIN AND SLEEP IN CHILDREN AND ADOLESCENTS}

\author{
Chair: Christine Chambers
}

\subsection{5 \\ MORE THAN A BAD NIGHT'S SLEEP: PAIN AND SLEEP IN CHILDREN \\ $\underline{C}$ Chambers $^{1}$, T Palermo $^{2}$, O Bruni ${ }^{3}, \underline{\text { O Ipsiroglu }}^{4}$ \\ ${ }^{1}$ Dalhousie University and IWK Health Centre, Halifax, Nova Scotia; ${ }^{2}$ Oregon Health \& Science University, Portland, Oregon, USA; ${ }^{3}$ University of Rome "La Sapienza", Rome, Italy; ${ }^{4} \mathrm{BC}$ Children's Hospital, Vancouver, British Columbia}

Our understanding of the roles of many biological, psychological, and social factors in childhood pain has grown tremendously in recent years. The relationship between one factor, disrupted sleep, and pediatric pain has, however, received relatively little attention from either researchers or clinicians. Reports that over half of children with pain-related conditions have difficulty sleeping have led researchers to consider the possible links between sleep and pain in children. There are a number of possible pathways of influence, including the obvious possibility that pain interferes with the quality and quantity of children's sleep, as well as the possibility that insufficient sleep contributes to increased daytime sensitivity to pain. Recent reviews of adult human and animal studies have concluded that there is reasonable evidence that deprivation or disturbance of sleep enhances pain sensitivity and can cause pain. Both the physiology of children and their most common pain conditions differ from those of adults, so parallel studies in pediatric populations need to be conducted, and are largely lacking. The purpose of this workshop is to provide a brief introduction to sleep in the context of pediatric pain, to review studies conducted on pediatric pain and sleep to date, to outline directions for future research, and to highlight sleep as a potential intervention target for children with chronic pain.

The workshop panel consists of an interdisciplinary, international panel of experts. Dr Chambers will begin the workshop by providing a brief overview of normal and abnormal sleep in children and adolescents and summarizing what is known about the relationship between sleep and pain from the relevant adult and animal literature. She will also present data documenting the impact of a sleep restriction protocol on healthy adolescents' self-reported and behavioural responses to experimentally induced cold pressor pain.

Dr Palermo will review data on subjective sleep patterns and sleep disturbances reported by adolescents with different forms of chronic pain and preliminary data on use of actigraphy as an objective assessment of sleep in this population. She will also present data demonstrating the adverse impact of sleep disturbances on daily functioning and quality of life in adolescents with chronic pain conditions. In addition, Dr Palermo will present longitudinal data examining changes in pain symptoms and sleep disturbances over time in adolescents receiving treatment for chronic pain and will present a conceptual model for understanding the bidirectional effects of pain and sleep.

Dr Bruni will use pediatric headache as an example of the complex ways that sleep and pain can interact. For example, sleep and headache are interrelated in many different ways: sleep can be a relieving factor or trigger factor for headache; sleep disorders can cause headache; headache patients are more prone to suffer from specific sleep disorders; and different forms of headache can be temporal sleep-related or sleep-stage linked. Dr Bruni will present data showing that children with migraine and tension-type headache have a higher prevalence of disorders of initiating and maintaining sleep, sleep breathing disorders, restless sleep, and daytime sleepiness. He will also present data documenting the temporal relations between sleep and pain among children with migraine. Data examining the relationship between headache and sleep quality in a large non-clinical sample of children and adolescents will also be reviewed. Finally, Dr Bruni will present the results of an intervention study employ- 
ing sleep hygiene among a group of children with migraine to determine the impact on duration, frequency, and severity of migraine attacks.

\subsection{2 \\ SLEEP DISTURBANCES IN CLINICAL POPULATIONS OF CHILDREN AND ADOLESCENTS WITH CHRONIC PAIN \\ T Palermo}

Department of Anesthesiology and Peri-Operative Medicine and Psychiatry, Oregon Health \& Science University, Oregon, USA

Disturbed sleep is a common complaint among children and adolescents with pain conditions. There is emerging evidence that these children and adolescents experience difficulty falling asleep, frequent night awakenings, early morning awakening, and excessive daytime sleepiness. Research using subjective and objective measures of sleep in children with chronic pain will be discussed. In particular, Dr Palermo will present data demonstrating the adverse impact of subjectively reported sleep disturbances on daily functioning and quality of life in adolescents with chronic pain conditions. Dr Palermo will also present preliminary data on the use of actigraphy as an objective assessment of sleep in adolescents with chronic pain, and will discuss advantages and disadvantages of this methodology. Last, Dr Palermo will highlight the importance of considering the etiology of sleep complaints using insomnia as an example. A conceptual model for understanding the bidirectional effects of pain and sleep will be discussed.

\section{RAPID COMMUNICATION WORKSHOP 1A - PHARMACOGENOMICS OF ANALGESIC DRUGS: SENSE OR NON-SENSE? \\ Chair: Dick Tibboel}

\subsection{1 \\ PHARMACOGENOMICS OF ANALGESIC DRUGS: SENSE OR NON-SENSE?}

D Tibboel ${ }^{1}$; S Anand ${ }^{2}$; L Van den Anker $^{3}$; B Anderson $^{4}$

${ }^{1}$ Erasmus MC - Sophia Children's Hospital, Department of Pediatric Surgery, Rotterdam, Netherlands; ${ }^{2}$ Arkansas Children's Hospital, Little Rock, Arkansas; ${ }^{3}$ Childrens National Medical Center, Washington, District of Columbia, USA; ${ }^{4}$ Oakland Children's Hospital, Oakland, New Zealand

BACKGROUND: Pharmaceutical agents are one of the most commonly identified causes of adverse events, resulting insignificant patient morbidity, mortality and excess medical costs. Critically ill children receive on average 10 different drugs during their stay at the pediatric intensive care unit, more than half prescribed unlicensed. Unlicensed drugs may be particularly unsafe, considering the lack of research done in these drugs. One possible cause of adverse drug reactions is genetic variation in how individuals metabolize drugs. The Human Genome Project herald new information to individualize drug therapy, called pharmacogenomics. In fact, pharmacogenomics may be one of the most immediate clinical applications of the Human Genome Project. Drugs frequently cited in adverse drug reaction studies, many of which are prescribed to critically ill children, are more often metabolized by drug metabolizing enzymes known to exhibit a genetic polymorphism in comparison to randomly selected drugs (59\% vs $7-22 \%$ ). Sedatives and analgesics are frequently prescribed to critically ill children and validated even in the smallest babies to evaluate their effect (pharmacodynamics). For these drugs, genetic polymorphisms have been found, explaining part of the variability in drug handling and effect. However, pharmacogenetic tests are currently performed rarely in clinical practice. When genetic variability is found in drug handling or effect, the ultimate question is 'does it matter?'. Currently, it is difficult to answer this question for drugs used in critically ill children as randomized control trials comparing genotype-based individualized drug therapy versus 'one size fits all' therapy are lacking. Due to the large number of patients needed for such trials, it is unlikely that these studies can be performed in this population. However, a list of criteria can be used to determine if pharmacogenetic testing may add in optimizing drug therapy. First, allelic frequency and
DOP . DO NOT COPY

prevalence of therapy failure or drug toxicity and the use of the drug in the patient population should all be high enough to warrant genetic testing. Attention should be paid to the prevalence of the polymorphic alleles in the patient population being treated since prevalence varies significantly among ethnic groups. Also, consequences of therapeutic failure or toxicity must be clinically relevant or lead to significant costs. Second, sufficient evidence exists to link the variant allele to clinical response. Third, the DNA assay is easy, fast and relatively inexpensive. Fourth, clinicians are able to interpret the results and use the information. If genetic variability appears a significant problem, alternative drugs may be considered, adverse events may be monitored more closely, or genotyping can be considered. Is such an approach already difficult for adults, as other factors like co-medication and disease state also contribute to interindividual variation in drug response, in children, also maturational processes impact drug handling and response. Hence, data from adults can not be extrapolated to critically ill children. Therefore, in order to reduce therapy failure or drug toxicity related to pharmacogenetic variation, an evaluation as described above needs to be carried out with regards to the prescription of analgesia in this population.

\subsection{2}

\section{PHARMACOGENETICS TO IMPROVE THE USE OF ANALGESIC DRUGS}

\section{IN van den Anker}

Pediatrics, Pharmacology \& Physiology, Children's National

Medical Center, Washington, District of Columbia, USA, and Pediatric Clinical Pharmacology, Erasmus MC - Sophia Children's Hospital, Rotterdam, the Netherlands

BACKGROUND: Developmental changes and pharmacogenetics are the main determinants of age-associated differences in pharmacokinetics (PK) and metabolism during human development. Recent developments have underlined the impact of pharmacogenetics of receptors and signal transducing pathways on the pharmacodynamics (PD) of pharmacological pain treatment.

PURPOSE: This talk will summarize clinically relevant mutations in drug metabolizing enzymes, transporters, and receptors to underscore the importance of incorporating pharmacogenetics into clinical trials of frequently used medicines for pain relief.

METHODS: The importance of pharmacogenetics for the PK and/or PD of morphine, ibuprofen, codeine and tramadol will be discussed using adult data from the literature and data generated in neonates and infants (Rotterdam, Louvain).

RESULTS: In neonates, pharmacogenetics ( $\mu$-opioid receptor, COMT) are of importance especially for the PD of morphine, whereas for codeine/tramadol (CYP2D6) already early in life it impacts PK. Polymorphisms in CYP2C8 and CYP2C9 might explain the relation with adverse drug events if using ibuprofen. There is a clear relation between development and the impact of pharmacogenetic background.

CONCLUSIONS: For the majority of medicines used for pain relief there is a lack of information on PK, PD and the role of genetics. Large clinical trials are needed to study efficacy and safety of frequently used analgesics, but these trials should incorporate the measurement of metabolites, renal function and genetic background to further optimize the use of these medicines in children of different ages. Ultimately this will lead to personalized medicines even in the youngest of our patients.

\subsection{3 PHARMACOGENOMICS - UNCERTAINTIES AND RELEVANCE B] Anderson Department of Anaesthesiology, University of Auckland, New Zealand}

Single nucleotide polymorphism (SNP) exist in children and contribute to adverse drug reactions (1), receptor response (2) and clearance variability $(3,4)$ but their impact, apart from a few well cited examples (eg, codeine and CYP2D6 [5]), remains uncertain. The benefits of gene testing above therapeutic drug monitoring (TDM) with Bayesian forecasting remains unproven $(6,7)$. 

Size, age and body composition are the three main covariates contributing
clearance variability of major analgesic drugs in children $(8,9)$. SNP plays little role in the metabolism of sedatives and analgesics prescribed in the smallest babies because the phenotype may have limited function in the first few years of life (10-12). An SNP is only important if it contributes $>50 \%$ metabolism, has an active metabolite, a steep dose-response relationship and a narrow therapeutic index. Few drugs fulfill these criteria. Most drug responses involve a large number of proteins regulated by multiple genes. Genotype does not equate with phenotype, environment concomitant therapy and disease have impact, and allele prevalence varies among ethnic groups. There are, for example over 60 CYP types identified. Only 6 have major influence and of these only 2D6 and 2C19 are relevant. Seeking an alternative to codeine in children removes impact from the most pertinent clinical SNP.

DNA assay may be easy, fast and relatively inexpensive, but its significance is questionable. Ethical considerations may preclude testing. TDM with Bayesian forecasting can individualize therapy, identify fast and slow metabolizers and correct for disease and other drug therapy. Many drugs used for analgesia are titrated to effect. Even nortriptyline (CYP2D6, $50 \mathrm{x}$ concentration variability) is monitored by TDM, effect and side effects. Pharmacogenomics in children will have some future relevance, but simpler issues such as clearance maturation data, pediatric formulations and palatability issues should dominate current thinking.

\section{REFERENCES}

1. Phillips KA, Veenstra DL, Oren E, Lee JK, Sadee W. Potential role of pharmacogenomics in reducing adverse drug reactions: A systematic review. JAMA 2001;286:2270-9.

2. Stamer UM, Bayerer B, Stuber F. Genetics and variability in opioid response. Eur J Pain 2005;9:101-4.

3. Fishbain DA, Fishbain D, Lewis J, et al. Genetic testing for enzymes of drug metabolism: Does it have clinical utility for pain medicine at the present time? A structured review. Pain Med 2004;5:81-93.

4. Flores CM, Mogil JS. The pharmacogenetics of analgesia: Toward a genetically-based approach to pain management. Pharmacogenomics 2001;2:177-94.

5. Fagerlund TH, Braaten O. No pain relief from codeine...? An introduction to pharmacogenomics. Acta Anaesthesiol Scand 2001;45:140-9.

6. Ensom MH, Chang TK, Patel P. Pharmacogenetics: The therapeutic drug monitoring of the future? Clin Pharmacokinet 2001;40:783-802.

7. Johnson JA. Drug target pharmacogenomics: An overview. Am J Pharmacogenomics 2001;1:271-81.

8. Bouwmeester NJ, Anderson BJ, Tibboel D, Holford NH. Developmental pharmacokinetics of morphine and its metabolites in neonates, infants and young children. $\mathrm{Br} \mathrm{J}$ Anaesth 2004;92:208-17.

9. Anderson BJ, van Lingen RA, Hansen TG, Lin YC, Holford NH. Acetaminophen developmental pharmacokinetics in premature neonates and infants: A pooled population analysis. Anesthesiology 2002;96:1336-45.

10. Allegaert K, Anderson BJ, Verbesselt R, et al. Tramadol disposition in the very young: An attempt to assess in vivo cytochrome P-450 2D6 activity. Br J Anaesth 2005;95:231-9.

11. Pariente-Khayat A, Rey E, Gendrel D, et al. Isoniazid acetylation metabolic ratio during maturation in children. Clin Pharmacol Ther 1997;62:377-83.

12. Kearns GL, Robinson PK, Wilson JT, et al. Cisapride disposition in neonates and infants: In vivo reflection of cytochrome P450 3A4 ontogeny. Clin Pharmacol Ther 2003;74:312-25.
RAPID COMMUNICATION WORKSHOP 1B ASSESSMENT OF PEDIATRIC PAIN: METHODOLOGICAL CONSIDERATIONS

\author{
Chair: Ignasi Clemente
}

2.402.1 QUALITATIVE METHODOLOGIES IN PEDIATRIC PAIN
ASSESSMENT: HOW THE DEPTH AND SUBTLETY OF
DETAIL CAN BE USED TO IDENTIFY LESS PERCEPTIBLE
FORMS OF PAIN AND SUFFRING

\section{$\underline{\text { Clemente }}$}

UCLA Center for the Study and Treatment of Pain, Pediatric Pain

Research Program, Department of Pediatrics, David Geffen School of Medicine at UCLA, Los Angeles, California, USA

As IASP's definition of pain underscores, "pain is always subjective." Thus, no one (researcher, clinician, or parent) can have a direct and exact access to a child's own subjective experience of pain and suffering. The subtle and nuanced observations obtained through qualitative methodologies (direct, participant, and deferred observation, as well as interviews) have helped researchers to (1) uncover unspoken or tacitly expressed forms of pain and suffering that otherwise might go undetected and therefore untreated, and to (2) account for different types of unexplained pain.

This talk, based on extensive fieldwork among pediatric cancer patients in Barcelona, Catalonia (Spain), explores one such instance: the significant amount of distress associated with the receipt of uncertainty and bad news by pediatric cancer patients. Such distress is observable in the question-answer exchanges during which pediatric cancer patients ask delicate questions on sensitive topics, and clinicians struggle between (a) full disclosure of bad or uncertain news and (b) partial withholding of information to spare the patients unnecessary anxiety and suffering. However, because patients interpret the clinicians' partial withholding of information as concealment of only bad and uncertain news, my analysis calls into question the effectiveness of partial disclosure as a communicative strategy to alleviate patient distress. Adolescent patients, in particular, frequently interpret partial disclosure as a smokescreen for bad news; and they show their distress in multiple tacit ways, such as repetitive questioning. A longitudinal and qualitative microanalysis of videotaped interactions and interviews with oncologists, patients, and parents thus reveals multiple tacitly expressed forms of pervasive distress and suffering, which are potential sources of intense physical and emotional suffering throughout the course of the illness, if left untreated or unaddressed. Implications for the integration of qualitative methodologies and findings in clinical research and work will be put forward and discussed.

\subsection{2}

\section{WHY CAN'T WE AGREE? THE DIVERGENCE OF SELF-REPORT, PARENT REPORT, AND STAFF REPORT OF PEDIATRIC PAIN}

$\underline{\text { LL Cohen }}$

Department of Psychology, Georgia State University, Atlanta, Georgia, USA

The simplest method of gauging children's pain is simply to ask them about it. In fact, self-report has been described as the gold standard of pediatric pain assessment. Given that parents often are required to advocate for their children's medical needs, and medical staff make the majority of decisions regarding diagnoses and treatment, their perspectives are important to consider too. As such, a number of measures have been developed to tap the perception of pain from the pediatric patient, the parent, and the medical staff. However, research has demonstrated that these individuals do not agree on what the child is experiencing and these measures are often not correlated. It is important to explore this phenomenon to determine: a) the strengths and weaknesses of child, parent, and staff rating scales; b) the reasons that the parties do not agree; and c) what information is revealed in these divergent views. A thorough examination of patient selfreports, parent ratings, and staff report of child pain will likely have 
clinical and research implications. This talk will explore different types of self-report, parent report, and staff report of pediatric pain, present data comparing the different perspectives from clinical trials, suggest interpretations for these findings, and discuss the implications of these issues.

\subsection{3}

\section{OBSERVATIONAL BEHAVIOUR ASSESSMENT: PARENT, STAFF, AND PEDIATRIC PATIENT INTERACTIONS \\ CM McMurtry}

Dalhousie University, Halifax, Nova Scotia

Observational measures of discrete behaviour by parents, staff, and children can provide a wealth of information unavailable through self-report ratings. For example, not only have parents been found to be inaccurate at reporting their behaviour during medical procedures, but parental behaviour also has a significant impact on a child's level of distress. In fact, maternal behaviour has been found to account for over half of the variance in child distress behaviour during immunization. But not all parent and medical staff behaviours are created equal: some caregiver behaviours are associated with child coping while others are linked with child distress. The creation of evidence-based treatment guidelines that reduce child distress and facilitate child coping during medical procedures requires a thorough understanding of the behaviours of and interactions between parents, staff, and pediatric patients. In her talk, Ms McMurtry will explore common observational measures and highlight important and unexpected findings these measures have produced. In addition, both the advantages and challenges of using observational scales will be discussed. Reassurance, which has been found to be the most common type of childdirected speech during medical procedures, will be one example used to illustrate the complexities involved in observational assessment of behaviour.

\section{RAPID COMMUNICATION WORKSHOP 1C - EXPERIMENTAL PAIN RESEARCH IN CHILDREN: RELIABILITY, VALIDITY, AND CLINICAL UTILITY \\ Chair: Jeroen Peters}

\subsection{1}

EXPERIMENTAL PAIN RESEARCH IN CHILDREN: RELIABILITY, VALIDITY, AND CLINICAL UTILITY IWB Peters $^{1,}{ }^{\text {CL von Baeyer }}{ }^{2}$

${ }^{1}$ Departments of Pediatric Surgery and Pediatric Anesthesiology, Erasmus MC - Sophia Children's Hospital, Rotterdam, Netherlands, ${ }^{2}$ Departments of Psychology and Pediatrics, University of Saskatchewan, Saskatoon, Saskatchewan BACKGROUND: Studies using experimental pain have provided relevant information about pain mechanisms, efficacy of (non) pharmacological interventions relieving pain, and the psychological variables affecting the pain experience. These type of studies, however, mainly concern adults.

PURPOSE: To learn about the principles and methodology of human experimental pain (models), their relevance for clinical practice, and the problems arising when conducting such tests in children.

METHODS: Thermal somatosensory testing and the cold pressor task are two techniques to induce experimental pain. In the first, a thermode is placed on the subject's skin. This thermode can induce thermal (pain) stimuli. Subjects are asked to respond to these stimuli either by pushing a button to stop the test or by reporting the level of perceived pain intensity. The second technique involves placing a hand or forearm in cold water, a stimulus that produces a slowly mounting pain of mild to moderate intensity and is terminated by voluntary withdrawal of the limb. Reliability, validity and feasibility of both techniques have been demonstrated in children. Methodological differences in the temperature, apparatus, and procedures may, however, contribute to conflicting results. In this presentation, the methodology of both techniques will be explained and guidelines for consistent administration will be provided.

\subsection{2}

ASSESSMENT OF CORTICAL POTENTIALS TO PAINFUL

STIMULI

\section{Hermann}

Department of Clinical and Cognitive Neuroscience, RuprechtKarls-Universität Heidelberg, Central Institute of Mental Health Mannheim, Germany

BACKGROUND: The measurement of pain-related brain responses has provided important insights into pain mechanisms and the development and maintenance of pain memories at the neuronal level. Yet, pain-related brain responses have rarely been studied in children even though they may help to elucidate developmental aspects of pain processing and may circumvent the problems associated with subjective-verbal pain report in children.

PURPOSE: One prerequisite for studying pain-induced brain responses using for example cortical evoked potentials or functional neuroimaging is the availability of a pain stimulus that can reliably be adjusted to the individual pain sensitivity and can be administered repeatedly with precise timing. In children, aspects such as the suitability of and familiarity with a pain stimulus as well as ethical considerations impose certain limitations on the availability of such stimuli.

METHODS AND RESULTS: In this workshop, the development and validation of an experimental protocol to obtain somatosensory evoked potentials in response to painful and nonpainful stimuli will be reported. A particular focus will lie on the choice of stimulation method and the comparison between children's and adults' responses using the same experimental design. In addition, the utility of the assessment of cortical evoked potentials to pain-related stimuli will be demonstrated based on data obtained in children suffering from migraine and recurrent abdominal pain.

\section{RAPID COMMUNICATION WORKSOP 1D - ANALGESICS AND ANALGESIC FAILURE IN THE CHILD WITH A COMPLEX DEVELOPMENTAL DISABILITY}

Chair: Tim Oberlander

\subsection{4}

\section{ANALGESICS AND ANALGESIC FAILURE IN THE CHILD} WITH A COMPLEX DEVELOPMENTAL DISABILITY

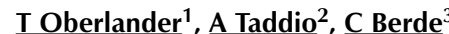

${ }^{1}$ Division of Developmental Pediatrics, Complex Pain Service, BC Children's Hospital, British Columbia; ${ }^{2}$ Scientist, Department of Pharmacy and Population Health Sciences, The Hospital for Sick Children, Toronto, Ontario; ${ }^{3}$ Pediatrics and Anesthesia, Division of Pain Medicine, Children's Hospital, Boston, Massachusetts, USA OVERVIEW: Cognitive, social and communication limitations associated with a complex developmental disability pose substantial challenges to managing pain in a child with a congenital (cerebral palsy) or acquired neurological injury (spinal cord injury). Even when appropriate pharmacological strategies are used, pain management is further complicated by the combination of the conditions inherent to the neural injury (ie, spasticity) or multiple medications used to manage the sequelae of the neurological impairment (ie, dislocated joints, gastrointestinal reflux).

Effective pain management requires identification of sources of pain, understanding of mechanisms that generate or mediate pain, a knowledge of pharmacological, enzymatic, genetic (pharmacogenetic and pharmacogenomic) variables as well as the neurodevelopmental condition itself. Medications needed to manage seizures, gastrointestinal reflux, infections, or comorbid psychiatric conditions can all have important and potentially serious implications for effective pharmacological approaches to pain management. Drug interactions can occur, leading to both toxicity or reduced availability (ie, increased drug metabolism) further contributing to significant morbidity and additional pain and suffering. Typical routes for drug administration are frequently not available and novel approaches 
need to be considered that include intravenous, topical and intrathecal techniques. Moreover, difficulties managing dislocated hips, spasticity or movement disorders that frequently accompany static or evolving neurological injury further compounds pain and suffering. Thus an understanding of key contextual, disease-related, biological and pharmacological variables is required to limit unwanted side effects and optimize pain management.

In this workshop we will use a case of a 10-year-old with choreoathetoid cerebral palsy, hip pain and multiple medications required for comorbid psychiatric and gastrointestinal disorders, to develop approaches to pain management. This will include review of the neurobiology of pain in the presence of a neurological impairment, implication of polypharmacy and appreciation of the interface between spasticity and pain. This workshop is intended for the health care professional working with children with a variety of developmental disabilities and will build on existing knowledge of child development, disabilities and pain management. General principles and approaches to pain management discussed in this workshop should be applicable across a variety of child health contexts.

This workshop will use a case-based approach to engage active participation to achieve the following objectives:

1. Understand neural impairment and pain: Discuss relationships between neurological impairment and pain in children with complex developmental disabilities (acquired and congenital) and the implications for successful pain management.

2. Apply pharmacological principles: Discuss analgesic pharmacology and factors that limit or promote successful pain management, including drug metabolism, pharmacogenetic factors, the impact of intercurrent illness (ie, infection), and drug-drug interactions. Understand the consequences of polypharmacy and implications this may have for successful pain management.

3. Develop clinical decision making approach: Applying a knowledge of the neurological conditions associated with complex developmental disabilities and analgesic options, develop approaches to clinical decision making that leads to management of combinations of pain, muscle spasticity and neurological conditions that compound pain in this context.

1. Tim Oberlander: Setting the scene: Analgesic failure in a child with a disability

- Why would our approach to pain management be different in a child with a neurological impairment?

- Review of basic neurological, pharmacological and contextual variables

2. Anna Taddio: Analgesic Pharmacology

- Basic principles of analgesic pharmacology

- Drug metabolism, pharmacogenetics and drug interactions associated with analgesic failure

3. Chuck Berde: Approaches to Clinical Decision Making

- Use of "n of 1" trial strategies

- Innovative therapies for spasticity and pain management: intrathecal therapy, Botox and other interventions.

- Postoperative pain management: What is different and what works managing postoperative pain in children with neurological impairments.

\section{RAPID COMMUNICATION WORKSHOP 2A - INFANTS IN PAIN AND THEIR CAREGIVERS: ADVANCING AND INTEGRATING RESEARCH AND PRACTICE \\ Chair: Rebecca Pillai-Riddell}

\subsection{1 \\ THEORETICAL CHALLENGES: CONCEPTUALIZING THE CAREGIVER AND INFANT IN PAIN \\ R Pillai Riddell}

York University/The Hospital for Sick Children, Toronto, Ontario

BACKGROUND: Infancy is marked by a profound sensitivity to noxious stimuli, more limited abilities to manage distress (both physically and cognitively), and a greater vulnerability to the long-term consequences of unrelieved pain. The adoption of a biopsychosocial model of infant pain requires an understanding of the infant that goes beyond stimulus intensity and into the psychosocial context in which an infant experiences pain. Owing to their complete dependence on their caregivers, an understanding of the dyadic context of the infant and caregiver is crucial. PURPOSE AND METHODS: A strong theoretical foundation is an important starting point, from which to understand infants in pain and their caregivers. While theoretical groundwork has been laid that postulates the reciprocal influences between an infant and a caregiver, specific work that explores the actual mechanisms for this influence is sparse. To expand the theoretical basis in which to explore infant pain, a brief introduction to classic developmental theory on behavioural control systems (attachment and caregiving) and its application to infant pain will be provided. New research that examines maternal and infant variables as predictors of infant pain reactivity will be presented and interpreted from an attachment perspective.

\subsection{2 \\ MEASUREMENT CHALLENGES: MEASURING THE CAREGIVER AND INFANT IN PAIN \\ LL Cohen}

Department of Psychology, Georgia State University, Atlanta, Georgia, USA

Infant pain has long been ignored, both in the clinical and research domains. Fortunately, a burgeoning body of research and clinical attention has begun to address this inequity. Accurate and reliable assessment is the foundation for all clinical and research efforts in infant pain. However, quantifying the nebulous phenomenon of infant pain poses a number of challenges. This talk will include an overview of the difficulties in assessing infant pain. Subsequently, a description of several of the measures of infant acute pain will be highlighted. Particular attention will be focused on behavioural and multidimensional scales. In addition to examining infant pain, evaluations of parent and medical staff behaviour during infants' painful medical events can reveal those adult behaviours that might help decrease infant anxiety and pain and those that might not be helpful. Measures of adults' behaviour during infants' painful procedures will be discussed along with findings from this literature. In addition, major challenges around quantifying and examining parent, nurse, and infant behaviour, and areas of future inquiry in this line of study will be detailed.

\subsection{3 DISSEMINATION CHALLENGES: TRANSLATING RESEARCH INTO PRACTICE FOR HOW TO CARE FOR THE INFANT IN PAIN}

\section{B Stevens}

\section{University of Toronto, Toronto, Ontario}

There has been exponential growth in infant pain research over the past two decades; however, the gap between research and best pain management practices remains wide. Although substantial research evidence has accumulated, and should compel health care providers to deliver optimal pain management, acute pain management practices in the pediatric clinical setting are rarely based on evidence and continue to be suboptimal. The challenge of adequately managing pain in infants has largely been attributed to individual knowledge deficiencies of health care providers. Attributing the persistent and suboptimal pediatric pain management practices to knowledge deficits, appears to be an oversimplification of a more complex issue. Rather than considering the persistence of inadequate acute pain management as a knowledge deficiency problem or the result of inadequate quantity or quality of research evidence, the core issue may be a failure to use knowledge effectively in practice. Therefore, the challenge is one of knowledge translation, not knowledge generation. Strategies to enhance knowledge translation must reflect the complexity of implementing evidence into the clinical setting through changes in health professional behaviour taking individual and contextual determinants of implementing evidence into practice, into account.

A brief review of an informative model of research utilization (Promoting 
Action on Research Implementation in Health Services - PARIHS)
(Kitson et al, 1998; Rycroft-Malone, 2004), will highlight the challenges of translating research on pain into practice. Specifically, attention will focus on the interplay between the level of evidence (eg, research, clinical experience, patient experience and local contextual information), context (eg, organizational culture) and facilitation (eg, interventions). Moreover, attention will be paid to the challenges associated with each key element of the model and the practical application of the model into clinical practice with infants. Examples of interactive knowledge translation strategies such as Evidence-based Practice In Change (EPIC) will be explored.

\section{RAPID WORKSHOP 2B - TOPICAL ANESTHETICS FOR PEDIATRIC PROCEDURES: NEW FRONTIERS \\ Chair: William Zempsky}

\subsection{2 \\ TOPICAL ANESTHETICS FOR PEDIATRIC PROCEDURES: NEW FRONTIERS}

WT Zempsky

Pain Relief Program, Connnecticut Children's Medical Center, Hartford, Connnecticut, USA

The ideal topical anesthetic for dermal procedures would be rapidly effective, inexpensive, with minimal local effects and negligible systemic absorption. Presently available topical anesthetics such as EMLA and LMX4 require 30 to 60 minutes to provide adequate anesthesia.

The stratum corneum is the primary barrier to the efficient delivery of anesthetics transdermally. Several new technologies have been developed which enable local anesthetics to penetrate the stratum corneum safely but more efficiently. These include iontophoresis, heat-augmented delivery, ultrasound-assisted delivery, and pressure-assisted delivery of local anesthetics. The mechanisms of these technologies, clinical efficacy and future potential will be discussed.

\begin{tabular}{c}
\hline RAPID COMMUNICATION WORKSHOP 2C - \\
PAIN ASSESSMENT IN CHILDREN: BEYOND \\
PAIN INTENSITY \\
Chair: Eufemia Jacob
\end{tabular}

\subsection{3}

\section{ASSESSMENT IN CHILDREN: BEYOND PAIN INTENSITY}

E Jacob $^{1}$, M Crandall ${ }^{2}$, LStinson $^{3}$, A Jordan $^{4}$

${ }^{1}$ Hematology/Oncology, Baylor College of Medicine, Houston, Texas; 'University of California Davis, Children's Hospital, Sacramento, California, USA; ${ }^{3}$ Chronic Pain Program, The Hospital for Sick Children, Toronto, Ontario; ${ }^{4}$ University of Bath \& Royal National Hospital for Rheumatic Diseases, Bath, United Kingdom

Pediatric pain measurement has progressed substantially over the last decade. A plethora of pain measures have been developed and validated for use with children and adolescents. The unique developmental needs across this wide age spectrum necessitates the use of different methods of pain assessment. The approaches to measuring pain in children include: self-report, observational behavioural and physiological. Given the definition of pain adopted by the International Association of Pain as an unpleasant sensory and emotional experience, it is most desirable to obtain and rely on self-report measures of pain when possible. Despite this definition, single-item ratings of pain intensity have been the most commonly used measures in pain research and clinical settings in children and adolescents. However, pain is a complex multidimensional experience comprising qualitative and affective components, in addition to pain intensity. Measures of pain location, quality, affect and pain's interference with aspects of everyday life might improve the evaluation and treatment of pain, especially in recurrent and chronic conditions.

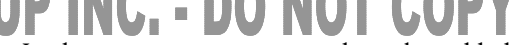

In this session, we examine the value added of self-report composite measures that tap into the multiple dimensions of the pain experience. The objectives of this workshop are to: (1) describe research on the use of a body outline diagram, pain quality word descriptors and a measure of pain interference in children with recurrent and chronic pains; (2) discuss the advantages and disadvantages of these measures in clinical practice and research; and (3) discuss further avenues of research in these areas to improve the assessment of pain in children and adolescents.

\subsection{1}

ASSESSMENT OF PAIN LOCATION USING BODY OUTLINES E Jacob

Hematology/Oncology, Baylor College of Medicine, Houston,

Texas, USA

Ms Jacob will examine how the body outline diagram has been used in the assessment of pain in children with sickle cell disease and Spanish speaking children with cancer.

The Adolescent Pediatric Pain Tool (APPT) is a multidimensional pain instrument for assessment of location, intensity, and quality. Children and adolescents ( 5 to 19 years) with sickle cell disease were asked to complete the APPT during hospitalization for acute painful episodes. Chest pain clearly delineated the sternal area and demonstrated that chest pain did not always imply lung (acute chest syndrome) or heart (myocardial infarction) involvement. Dark and light shadings represented variations in intensity that were not reflected in a numerical pain rating scale. While pain intensity ratings did not show a significant decrease throughout hospitalization, the total amount of surface area marked on the body outline diagram showed a significant decrease by about $50 \%$. Therefore, ratings of pain intensity may remain moderately high, but the pain location surface area and the spatial distribution of the pain may change dramatically.

Children with cancer may have difficulty quantifying their pain using numerical rating scales, but they are able to communicate the extensiveness of their pain by marking the body outline diagram. In Spanish speaking children with cancer $(n=25 ; 8$ to 12 years) pain was unrecognized and untreated, because clinicians were predominantly English speaking and were not able to assess pain in patients who spoke Spanish. These children however, were able to use the body outlines to communicate the location and extensiveness of their pain. The marked body outline diagram represents a universal language and can be used to encourage non-English speaking patients, or culturally diverse patients to communicate the location, spatial distribution and extensivenesss of their pain.

\subsection{2 \\ PAIN QUALITY AS DESCRIBED BY ADOLESCENTS AFTER TRAUMATIC BLUNT INJURY \\ $M$ Crandall \\ University of California Davis, Children's Hospital, Sacramento, California, USA}

Margie Crandall advocates for the use of a multidimensional assessment tool in children with complex pain. She will describe the use of a word list depicting pain quality to assess the pain experience of adolescents after a traumatic blunt injury. Her research shows that children were able to talk about the different dimensions of their pain experience using word descriptors that prompted them in talking about the pain.

The word descriptor list of the Adolescent Pediatric Pain Tool contains 67 words representing sensory, affective, evaluative and temporal dimensions of pain. In this qualitative study, adolescents (11 to 17 years) were asked to describe their experience after an acute blunt traumatic injury (BTI) during hospitalization. They circled the words that described the quality of their current pain and added words that were not on the list. They were encouraged to talk more about the words they selected to describe the quality of their pain and their experiences at the scene of injury, in the emergency department, and during hospitalization. Although similar words were selected, the words represented different meanings and different cumulative present pain experiences for each adolescent. Multiple aspects of their BTI experience were revealed and reflected the variations in the quality of pain specific to injury, procedural sites, pain 
with mobility, health status and reactions to injury, and subsequent procedures. Adolescents reported a therapeutic benefit to describing the quality of their pain. They were able to describe the individual meaning of their traumatic events. The use of the word list facilitates clinicians understanding of adolescents pain experiences, etiologies of pain, influencing factors and psychological distress associated with BTI. The quality word descriptors (affective, sensory, evaluative, temporal) may be useful in guiding the development and implementation of individualized nonpharmacological, psychosocial, cognitive behavioural, and selfinitiated pain alleviating strategies. Further evidence supporting the clinical usefulness of the word list to assess adolescents' individual and changing meanings of pain reflecting the effectiveness of therapeutic interventions is needed.

\subsection{3 \\ E-OUCH ELECTRONIC PAIN DIARY: MEASURING PAIN INTENSITY, UNPLEASANTNESS AND INTERFERENCE IN CHILDREN WITH ARTHRITIS}

\section{Stinson}

Chronic Pain Program, The Hospital for Sick Children, Toronto, Ontario

Jennifer Stinson's doctoral research focused on the development and psychometric testing of a multidimensional electronic diary for the assessment of chronic pain in adolescents.

The e-Ouch electronic pain diary is an innovative instrument for measuring the multiple dimensions of pain. The aspects of the pain experience include: (1) sensory (eg, intensity, quality, and location of pain sensations), (2) affective (eg, emotional effects using word descriptors and unpleasantness using visual analogue or "generic faces" scales), and (3) cognitive (eg, perception of pain's interference with daily functioning and overall quality of life) dimensions. Adolescents (9 to 12 years) with arthritis completed three daily pain ratings (upon waking, after school and before bed) using the e-Ouch electronic pain diary. They reported mild to moderate pain intensity as well as mild levels of pain unpleasantness. Pain interfered with activities of daily living, and with sleep, mood, walking, relationships with friends/family, and enjoyment of life. The participants, on average, reported mild stiffness, moderate levels of fatigue, and two painful joints (range 0-5) per diary entry, with the most commonly affected joints being the knees. RMANOVA was conducted to determine if there were significant differences in e-Ouch pain indices across the three points in time (morning, afternoon, and evening). There were no significant group $\times$ time effects.

All participants were very satisfied using the e-Ouch diary. The e-Ouch electronic pain diary may be crucial for: (1) understanding the day-to-day variability of pain and its impact on health-related quality of life, (2) selecting and evaluating the effectiveness of a broad range of painrelieving interventions, (3) identifying the underlying mechanisms of action of pain-relieving treatments, and (4) examining the correlates of pain and pain-related disability in adolescents.

\section{RAPID COMMUNICATION WORKSHOP 2D - PREDICTORS OF CHILD RESPONSE TO A PAINFUL MEDICAL PROCEDURE WHEN DISTRACTION IS PROVIDED BY A PARENT Chair: Kristen Hanrahan}

\subsection{1 CORTISOL RESPONSIVITY AND PARENT DISTRACTION DURING A MEDICAL PROCEDURE}

\section{K Hanrahan, AM McCarthy, D Schutte}

University of lowa, Iowa, USA

Each year, more than 4 million children in the United States require medical procedures. Distraction is effective in reducing pain and distress in these children and can be provided by parents. However, distraction does not work for some children, and some parents are not effective distraction coaches. Clinicians need to be able to predict children's individual responses to parent distraction during a painful procedure to minimize distress, plan interventions, and allocate resources. A multisite research project was conducted to identify factors that predict which children benefit from parent distraction coaching during a painful procedure. An experimental design tests an intervention teaching parents to use distraction coaching vs routine care. Participants were 542 children, 4-10 years of age undergoing an intravenous (IV) catheter placement. One measure of child response, salivary cortisol, was included to further our understanding of children's physiological response to stressful, painful stimuli. Salivary samples were collected by a standardized procedure using a salivary stimulant and the passive drool method. Extraneous variables, such as exogenous steroids, were identified and controlled. Two samples from each child were collected in clinic (on the day of the IV insertion) and two baseline samples collected at home are compared. Results include normal salivary cortisol values obtained on the baseline, nonprocedural day. The response pattern is similar to the circadian pattern in adults, decreasing from early morning to mid-afternoon. Group median and individual change scores of cortisol levels pre- and postprocedure, and between the day of the procedure and the baseline day will be compared. Cortisol responsivity will be used to identify predictors of child response to an IV insertion and to evaluate the effectiveness of the distraction intervention. Biological measures, such as salivary cortisol, can provide an important contribution to understanding distress response and identifying the appropriate interventions.

\subsection{2}

\section{GENETIC PREDICTORS OF CHILD RESPONSE}

\section{K Hanrahan, AM McCarthy, D Schutte}

\section{University of lowa, lowa, USA}

Children undergoing venipuncture demonstrate variable levels of pain and distress, despite the use of topical anesthetics. A multisite research project was conducted to identify factors that predict which children benefit from distraction coaching by parents during a painful procedure. An experimental design tests an intervention teaching parent to use distraction coaching vs routine care. Subjects were 542 children, 4-10 years of age undergoing an intravenous (IV) catheter placement. The integration of molecular genetics approaches into this project provides a unique and valuable opportunity to examine the role of genes with complex and variable behavioural phenotypes, such as pain and distress. For the molecular genetic aim of this project, a systematic review of the literature was undertaken to identify and prioritize a pool of candidate genes for pain and distress. Cheek swabs for DNA extraction were collected from the children and available from the biological parents. DNA from children and their parents are genotyped, using Taqman and standard PCR techniques, for one single nucleotide polymorphism in each of the 14 candidate genes. Analysis is underway to examine the relationship between genotype and individual measures of child response (eg, child self-report of pain) as well as the CDS. This workshop will present findings from Transmission Disequilibrium Test (TDT) analysis, a family-based Chi-square approach to determine the extent of transmission distortion from parent to children of high and low distress phenotypes. Results of additional group comparisons between genetic variants and child outcomes will also be reported. The identification of genetic factors that influence pain response will contribute to more robust predictive models, guiding the provision of more specified, tailored, and effective pharmacological and biobehavioural interventions.

\subsection{3 IDENTIFYING PREDICTORS OF CHILD RESPONSES TO A PROCEDURE WHEN PARENT DISTRACTION IS PROVIDED}

\section{K Hanrahan, AM McCarthy, D Schutte} University of lowa, lowa, USA

The experimental study described, Predicting Children's Responses to Distraction from Pain, was carried out in the outpatient clinics of three Midwest children's hospitals. Participants included 540 children, 4 to 10 years of age, having an intravenous (IV) catheter insertion, and one parent self-designated to provide support, randomly assigned to a control group (routine care) or an intervention group (instructions for 
distraction coaching). Standardized instruments were used to collect data on child characteristics (anxiety VAS, Pediatric Behaviour Scale, Dimensions of Temperament, Child Behaviour Style Scale, Child/Parent Coping Questions, Severity of Illness Scale, genotype), parent characteristics (Parenting Dimensions Inventory, State-Trait Anxiety Scale), and procedural variables. Child outcome measures include behavioural coding of child distress from videotapes of the IV insertions (Observational Scale of Behavioural Distress - Revised), salivary cortisol, child report of pain (Oucher Scale), and parent report of child distress.

Factors that predict child outcome response (child characteristics, parent characteristics, and procedural variables) will be analyzed for the entire group, for children who are not provided distraction coaching, and for children provided an adequate level of distraction coaching by their parent. The outcome measures will be tested separately and in combination, including a composite distress score (CDS) of the four outcome measures. In preliminary analyses of the first 200 subjects, the CDS (excluding cortisol) was calculated using a principal components analysis. The mean and standard deviation of the CDS z scores were used to rank the first 200 subjects into high, medium and low distress groups. Significant predictors were child state anxiety $(\mathrm{P}=0.001)$, parent state and trait anxiety ( $\mathrm{P}=0.035$ and 0.026 , respectively), and the child's history of distress during procedures $(\mathrm{P}=0.002)$. An inconsistent parenting style approached significance $(\mathrm{P}=0.062)$. Regression analyses will be used to further develop predictive models of distress with results from the 540 subjects.

\section{RAPID COMMUNICATION WORKSHOP 2E - PUTTING A NEW FACE ON FACE SCALES Chair: Carl von Baeyer}

\subsection{5}

PUTTING A NEW FACE ON FACES SCALES

CL von Baeyer ${ }^{1}$, IE Beyer ${ }^{2}$, C Chambers ${ }^{3}$

${ }^{1}$ Departments of Psychology and Pediatrics, University of Saskatchewan, Saskatoon, Saskatchewan; ${ }^{2}$ School of Nursing, University of Missouri - Kansas City, Kansas City, Missouri, USA; ${ }^{3}$ Departments of Pediatrics and Psychology, Dalhousie University, Halifax, Nova Scotia

Faces scales offer major advantages over numerical, visual analogue, and verbal rating scales for self-report of pain intensity, especially for children 4 to 8 years of age. Since the first faces scales were created in the 1980s, much has been learned about their psychometric properties. Numerous faces scales have been created, leading to questions about their comparability and utility. The presenters will consider and debate the relative advantages and disadvantages of existing faces scales and provide suggestions on future directions for research and dissemination of knowledge, including the following issues.

\subsection{1}

\section{FACES SCALES: INTERPRETATION, VALIDATION AND DISSEMINATION}

\section{CL von Baeyer}

Departments of Psychology and Pediatrics, University of Saskatchewan, Saskatoon, Saskatchewan

(12) Interpretation of scores, and minimum clinically significant difference (MCSD) in pain. (13) Use and understanding of the $0-10$ common metric. (14) Nature of data required to demonstrate validity and responsivity. (15) Screening for the child's ability to understand and complete the self-report task. (16) World wide dissemination of adequate tools: ease and economy of reproduction and distribution

The objectives of the workshop are to enable participants to make informed decisions as to choice, administration, and interpretation of faces scales, and to discuss directions for future development of this approach to pain assessment.

\subsection{2}

FACE SCALES: FORMAT AND ADAPTATION TO DIVERSE

\section{CULTURES}

\section{IE Beyer}

School of Nursing, University of Missouri - Kansas City, Kansas City, Missouri, USA

(1) Faces in comparison with other types of scales. (2) Use of cartoon versus photographic faces. (3) Vertical versus horizontal presentation. (4) Size of presentation to the child (wallet card up to poster size). (5) Use of colour in reproduction of the scale. (6) Adaptation of faces and instructions to different ethnic groups and cultures.

\subsection{3}

\section{FACES SCALES: ANCHORS, INSTRUCTIONS, AND CLINICAL UTILITY}

\section{CT Chambers}

Departments of Pediatrics and Psychology, Dalhousie University, Halifax, Nova Scotia

(7) Top and bottom pictorial anchors (smiling, neutral, and crying faces). (8) Top and bottom verbal anchors. (9) Verbal instructions for administration. (10) Role of adult and child preference (which may depend on cuteness rather than suitability for measurement of pain). (11) Utility of faces scales in clinical practice.

\section{PLENARY 2 - PHARMACOLOGY: WHAT'S NEXT?}

\subsection{1 NEUROPATHIC MECHANISMS IN PEDIATRIC PAIN $M$ Fitzgerald Department of Anatomy \& Developmental Biology, University College London, London, United Kingdom}

Neuropathic pain occurs in children but the incidence is lower than in adults. Considerable postnatal development occurs within nociceptive pathways, marked by anatomical, pharmacological and physiological 'finetuning' over the first postnatal weeks (Fitzgerald, 2005b; Fitzgerald, 2005a) and central mechanisms that underlie neuropathic pain in the adult are unlikely to be fully functional in the juvenile animal. Our recent study of two models of peripheral nerve injury revealed that mechanical allodynia, a characteristic sign of peripheral nerve injury-induced neuropathic pain did not develop if the injury was performed before three weeks of age. Importantly, pain symptoms were never detected in these rats even when they reached the age at which injury would normally evoke mechanical allodynia (Howard et al, 2005).

The lecture will discuss recent research on the central mechanisms underlying neuropathic pain and how these may develop in infants and children. Two mechanisms will be discussed in detail (i) the role of neuronal inhibitory processing in neuropathic pain circuits (Baccei and Fitzgerald, 2004; Bremner et al, 2006; Hathway et al, 2006) and (ii) the proliferation and activation of spinal microglia in the pathogenesis of neuropathic pain following peripheral nerve injury (Moss et al, 2006).

\section{REFERENCES}

Baccei ML, Fitzgerald M (2004) J Neurosci 24:4749-4757.

Bremner L, Fitzgerald M, Baccei ML (2006) J Neurophysiol (in press). Fitzgerald M (2005) Nat Rev Neurosci 6:507-520.

Hathway G, Harrop E, Baccei M, Walker S, Moss A, Fitzgerald M (2006) Eur J Neurosci 23:112-118.

Howard RF, Walker SM, Mota PM, Fitzgerald M (2005) Pain 115:382-389.

Moss, A, Beggs S, Hathway GJ, Salter MW, Fitzgerald M (submitted). 
2.601.2

\section{NON-STEROIDAL ANTI-INFLAMMATORY DRUGS: A PHARMACOKINETIC-PHARMACODYNAMIC WASTELAND IN KIDS}

\section{BJ Anderson}

Anaesthesiology, University of Auckland, Auckland, New Zealand The NSAIDs are commonly used in children for antipyresis, antiinflammatory and antinoceceptive effects. There are few pharmacodynamic (PD) studies investigating NSAID analgesia in children. In contrast, pharmacokinetic (PK) knowledge is rapidly expanding. NSAID adult clearances are reached within the first year of life. Volume of distribution is increased in the neonatal period. PK parameter estimate variability is large, partly attributable to covariate effects of age, size and pharmacogenomics. Ibuprofen, for example, is metabolized by the CYP 2C9 and 2C8 subfamily, and functional polymorphism of the gene coding for CYP2C9 has been described. Stereoselectivity also exists; R- and S-ibuprofen halflives were about 10 hours and 25.5 hours in premature neonates.

Dosing is tempered by concerns about toxicity. Renal function is reduced $20 \%$ after NSAID use for PDA closure in premature neonates, and there is no increased frequency of intraventricular haemorrhage or change in cerebral blood volume, change in cerebral blood flow, or tissue oxygenation index. NSAID exacerbated respiratory disease (ERD) is more a disorder of adults but exacerbations in children and teenagers have been reported. ERD is a concern in 1 in 3 teenagers with severe asthma and coexistent nasal disease. Bleeding time is usually slightly increased, but it remains within normal limits in children with normal coagulation systems. Ketorolac has been used to treat pain after congenital heart surgery. A Cochrane review has established that even after tonsillectomy, NSAIDs did not cause any increase in bleeding that required a return to theatre in children. There was significantly less nausea and vomiting with NSAIDs compared to alternative analgesics, suggesting their benefits outweigh their negative aspects.

The future of COX-2 selective inhibitors in children is uncertain following reports of atherothrombosis in adults. Future benefits may be derived from nitric oxide releasing NSAIDs that have increased potency and reduced side effects.

\section{REFERENCES}

1. Litalien C, Jacqz-Aigrain E. Risks and benefits of nonsteroidal anti-inflammatory drugs in children: a comparison with paracetamol. Paediatr Drugs 2001;11:817-58.

2. Anderson BJ. Comparing the efficacy of paracetamol and NSAIDs in children. Pediatr Anaesth 2004;14:201-17.

3. Van Overmeire B, Touw D, Schepens PJ, et al. Ibuprofen pharmacokinetics in preterm infants with patent ductus arteriosus. Clin Pharmacol Ther 2001;70:336-43.

4. Smyth JM, Collier PS, Darwish M, et al. Intravenous indomethacin in preterm infants with symptomatic patent ductus arteriosus. A population pharmacokinetic study. Br J Clin Pharmacol 2004;58:249-58.

5. Garcia-Martin E, Martinez C, Tabares B, et al. Interindividual variability in ibuprofen pharmacokinetics is related to interaction of cytochrome P450 2C8 and 2C9 amino acid polymorphisms. Clin Pharmacol Ther 2004;76:119-27.

6. Gregoire N, Gualano V, Geneteau A, et al. Population pharmacokinetics of ibuprofen enantiomers in very premature neonates. J Clin Pharmacol 2004;44:1114-24.

7. Gaitan G, Herrero JF. Subanalgesic doses of dexketoprofen and HCT-2037 (nitrodexketoprofen) enhance fentanyl antinociception in monoarthritic rats. Pharmacol Biochem Behav 2005;80:327-32.

8. Palmer GM. A teenager with severe asthma exacerbation following ibuprofen. Anaesth Intensive Care 2005;33:261-5

9. Lesko SM. The safety of ibuprofen suspension in children. Int J Clinical Practice. Supplement. 2003:50-3.

10. Allegaert K, Cossey V, Debeer A, et al. The impact of ibuprofen on renal clearance in preterm infants is independent of the gestational age. Pediatr Nephrol 2005;20:740-3.

11. Allegaert K, Anderson BJ, Cossey V, et al. Limited predictability of amikacin clearance in extreme premature neonates at birth. Br J Clin Pharmacol 2005;61:39-48.

12. Gupta A, Daggett C, Drant S, et al. Prospective randomized trial of ketorolac after congenital heart surgery. J Cardiothorac Vasc Anesth 2004;18:454-7.

13. Ment LR, Vohr BR, Makuch RW, et al. Prevention of intraventricular hemorrhage by indomethacin in male preterm infants. J Pediatr 2004;145:832-4.

14. Cardwell M, Siviter G, Smith A. Non-steroidal anti-inflammatory drugs and perioperative bleeding in paediatric tonsillectomy. Cochrane Database Syst Rev 2005:CD003591.

15. Naulaers G, Delanghe G, Allegaert K, et al. Ibuprofen and cerebral oxygenation and circulation. Arch Dis Child Fetal Neonatal Ed 2005;90:F75-6.

16. Sheeran PW, Rose JB, Fazi LM, et al. Rofecoxib administration to paediatric patients undergoing adenotonsillectomy. Paediatr Anaesth 2004;14:579-83.

17. Joshi W, Connelly NR, Reuben SS, et al. An evaluation of the safety and efficacy of administering rofecoxib for postoperative pain management. Anesth Analg 2003;97:35-8.

18. Jones SF, Power I. Editorial I: Postoperative NSAIDs and COX-2 inhibitors: cardiovascular risks and benefits. $\mathrm{Br} J$ Anaesth 2005; 95:281-4.

19. Krotz F, Schiele TM, Klauss V, et al. Selective COX-2 inhibitors and risk of myocardial infarction. J Vascular Res 2005;42:312-24

20. Levin RI. Theriac found? Nitric oxide-aspirin and the search for the universal cure. J Am Coll Cardiol 2004;44:642-3.

\subsection{3}

\section{THE NATURE AND NURTURE OF PAIN}

JS Mogil

Department of Psychology and Centre for Research on Pain, McGill University, Montreal, Quebec

Genetic approaches attempt to explain variability in biological systems, and pain is associated with much variability, including in the propensity to develop painful pathologies. Genetic linkage mapping efforts in mice and targeted genetic association studies in humans are beginning to identify those genes underlying the considerable variability noted in these traits. In our laboratory, we have recently uncovered a number of genes associated with variability in the response to thermal and inflammatory nociception. These efforts may lead to novel clinical treatments for pain and/or facilitate the patient-centred, individualized treatment of pain using current pharmaceuticals. In addition, we are now paying greater attention to the identification of laboratory environmental factors that affect pain behaviour in the mouse. We have very recently observed modulation of pain in the mouse by purely social factors. These data can be interpreted as providing evidence for the existence of empathy for pain in this mammalian species.

WORKSHOP 2A - PEDIATRIC PALLIATIVE CARE - THE CHILD'S PERSPECTIVE

Chair: Lonnie Zeltzer

\subsection{1 \\ PEDIATRIC PALLIATIVE CARE RESEARCH FROM THE PERSPECTIVE OF THE CHILD: HOW CAN WE SUCCEED? \\ Wolfe \\ Pediatric Palliative Care, Children's Hospital Boston and the Dana-Farber Cancer Institute, Boston, Massachusetts, USA}

INTRODUCTION: Palliative care strives to maximize the physical, emotional and spiritual needs of children with life-threatening illnesses and their families. High-quality palliative care is now an expected standard, and research in this field has been established as a priority. To date, much of the research has been retrospective and from the perspective of parents and providers.

OBJECTIVE: To assess the feasibility of conducting a randomized controlled supportive care trial (RCT) in children with advanced cancer. 
METHODS: Using a randomized design we are piloting an intervention, feeding back symptom and quality of life (QOL) data collected with a computerized survey, the Pediatric Quality of Life and Evaluation of Symptoms Technology (PediQUEST), as a component of the routine care of children with advanced cancer. Children 5 years old and above are asked to complete PediQUEST on their own at most once a week for the 3 to 9 month evaluation period. Parents of children 2 to 4 years old or of children who are unwilling to complete PediQUEST, complete PediQUEST on the child's behalf. Among children randomized to the intervention arm, we provide immediate provider and family feedback about symptom and QOL scores and generate electronic mail notifications alerting providers to high levels of distress. To assess feasibility, all subjects approached for enrollment are invited to complete a one-time "Consent survey". All enrolled subjects are also asked to complete a "Participation survey" after completing the study or dropping-out, to explore determinants of attrition.

RESULTS: We will present preliminary data regarding the feasibility of conducting this RCT, including proportion of eligible patients referred to the study by oncologists, recruitment and attrition rates, child versus parent completion rates, as well as data from consent and attrition surveys. CONCLUSIONS: Findings will inform the development of future RCTs among children with advanced illness.

\subsection{2 \\ UNDERSTANDING COMMUNICATION ABOUT SYMPTOMS IN CHILDREN WITH LIFE-THREATENING ILLNESSES: A QUANTITATIVE AND QUALITATIVE APPROACH TOWARD EXAMINING THE CHILD'S PERSPECTIVE}

\section{$\underline{\text { E Evan }}$}

UCLA Pediatric Pain Program, David Geffen School of Medicine at UCLA, Los Angeles, California, USA

INTRODUCTION: Pediatric palliative care aims to improve the quality of life of children with life-limiting conditions and their families through the prevention and relief of suffering. Information regarding the symptoms experienced by the child as well as the child's overall quality of life is predominantly obtained from the perspective of the primary caregiver(s) and/or the health care team, thus it is important that these proxy reporters have an accurate understanding of what symptoms the child is actually experiencing. OBJECTIVE: To better understand the nature of the symptoms experienced by children and young adults with critical illnesses through child and caregiver self-report methods using a quantitative and qualitative approach. METHODS: A multihospital site pediatric symptom communication study was conducted with children with life-threatening illnesses and their primary caregivers. Twenty-three children between $7-22$ years old with a life-limiting condition estimated to have a $<20 \%$ chance of survival beyond 3 years and their primary caregivers were administered quantitative measures regarding the child's symptom experience. Participants were then interviewed about the child's symptoms and overall illness experience utilizing a structured qualitative narrative format.

RESULTS: A discussion of the preliminary results derived from both quantitative and qualitative approaches utilized within the study design will take place, highlighting the unique contributions of each method. Empirical findings regarding factors affecting symptom control and management using case examples to highlight child and caregiver experiences in facing a child's life-limiting illness will then be presented. CONCLUSIONS: The study's findings regarding the child's symptom experience will be utilized to suggest future recommendations for pediatric palliative care.

\subsection{3 \\ AWARENESS OF CHILD'S IMPENDING DEATH TO CANCER: A NATIONWIDE FOLLOW-UP OF BEREAVED PARENTS}

U Kreicbergs

Dana Farber Cancer Institute/Children's Hospital Boston, Boston, Massachusetts and Karolinska Institutet, Stockholm, Sweden RESEARCH QUESTION: To support parents with seriously ill children in a long-term perspective we need to know if awareness of child's impending death may facilitate their long-term well being. Does early awareness in parents of their child's impending death facilitate psychological recovery? We investigated how and when parents gain intellectual awareness and emotional preparedness for their child's impending death. DESIGN: A population-based study was conducted using a mailed survey to a national-wide sample of bereaved parents in Sweden.

DATA COLLECTION: 449 parents (80\%) who had lost a child due to malignancy 4 to 9 years earlier answered an anonymous questionnaire. Questions included the duration of time before the child's death that they had been intellectually and emotionally aware of the child's death.

ANALYSIS METHODS: We assessed the proportion of parents reporting intellectual awareness and emotional preparedness over the course of the disease. Potential predictors of gaining late emotional preparedness and intellectual awareness were assessed in terms of relative risks (RR).

RESULTS: 191 parents out of 419 (46\%) reported not having observed their child to be aware of imminent death. Overall, 195 parents (45\%) reported late emotional preparedness ranging from a few hours or even less: 110 of 247 mothers (45\%) and 85 of 186 fathers (46\%). Mothers not informed about the fatal condition in their child were at increased risk of late emotional preparedness (RR 1.5, 95\% CI 1.1-2.1) as well as intellectual awareness (RR 3.5, 95\% CI 2.3-5.5) compared with informed mothers. Corresponding figures for fathers were 1.4 (95\% CI 1.0-2.1) and 2.9 (95\% CI 1.8-4.5).

IMPLICATIONS: Pediatric oncology health care staff have a central role in promoting intellectual awareness and emotional preparedness for the child and the parents' by making a clear transition to palliative care and providing accurate information on the child's condition.

\section{WORKSHOP 2B - TIES THAT BIND: MATERNAL ANALGESIA FOR PROCEDURAL PAIN IN NEONATES \\ Chair: Celeste Johnston}

\subsection{2}

\section{THE TIES THAT BIND: MATERNAL ANALGESIA FOR} PROCEDURAL PAIN IN NEONATES

\section{Johnston $^{1}$, R Carbajal $^{2}$, E Blass ${ }^{3}$}

${ }^{1}$ School of Nursing and Centre for the Study of Pain, McGill University, Montréal, Québec; ${ }^{2}$ Centre National de Ressources de lutte contre la Douleur, Hôpital Armand Trousseau, Paris, France; ${ }^{3}$ Department of Psychology, University of Massachusetts, Amherst, Massachusetts, USA

Maternal caring is comprised of several components, one important one being to provide comfort to and protect the infant from stress. Neonates, especially preterm neonates, who undergo painful procedures for diagnostic or therapeutic purposes, have typically been removed from the comfort potential of their mothers. This workshop will present studies on the efficacy of two maternal comfort interventions, kangaroo care and breastfeeding, and as well present some ideas to explain the mechanism underlying their effect.

\subsection{1}

\section{KANGAROO CARE}

\section{Johnston}

School of Nursing and Centre for the Study of Pain, McGill University, Montreal, Quebec

Kangaroo Care (KC) was initially incorporated into neonatal intensive 
care in the developing world as an alternative to incubators to maintain body heat. Serendipitous findings indicated that it might also serve as a comfort mechanism. The initial study using $\mathrm{KC}$ for pain control was with full term neonates (1), and subsequently two other studies have demonstrated its efficacy for preterm neonates (2,3). A more recent study with infants less than 32 weeks gestational age will be presented which also demonstrates the efficacy of $\mathrm{KC}$ in very premature neonates, the most significant finding being a reduction in time to recovery of more than one minute. A study exploring the effect of adding mother's voice, rocking, and singing which found no added value to usual $\mathrm{KC}$ will be presented. It is likely that it is the swaddling, warmth, and familiarity of the mother that contributes to the efficacy of $\mathrm{KC}$ for pain, although this requires further exploration.

\section{REFERENCES}

1. Gray L, Watt L, Blass EM. Skin-to-skin contact is analgesic in healthy newborns. Pediatrics 2000 Jan;105:e14.

2. Johnston CC, Stevens B, Pinelli J, et al. Kangaroo care is effective in diminishing pain response in preterm neonates. Arch Pediatr Adolesc Med 2003;157:1084-8.

3. Ludington-Hoe SM, Hosseini R, Torowizc DL. Skin-to-skin contact (Kangaroo Care) analgesia for preterm infant heelstick. AACN Clin Issues 2005;16:373-87.

\subsection{2}

\section{BREASTFEEDING ANALGESIA}

\section{R Carbajal}

Centre National de Ressources de lutte contre la Douleur, Hôpital Armand Trousseau, Paris, France

Breastfeeding maintained throughout a procedure has been shown to be a potent analgesic to relieve procedural pain in term neonates. Two studies on breastfeeding analgesia will be presented. First, a randomized controlled trial carried out in full term infants who were held and breastfed by their mothers during heel lance and blood collection procedures showed crying and grimacing reductions by $91 \%$ and $84 \%$, respectively, compared with control infants receiving the standard hospital care of being swaddled in their bassinets levels during the blood collection (1). Heart rate was also substantially reduced by breastfeeding. The second study was a prospective randomized controlled trial that included term newborns undergoing venipunctures (2). It showed that breastfeeding is extremely efficacious for the prevention of procedural pain in term neonates. The analgesic efficacy of breastfeeding was similar to that obtained with sweet solutions plus non-nutritive sucking.

Breastfeeding antinociceptive mechanisms are probably complex and the different components of breastfeeding, such as touch, temperature, odor or milk taste may all play a role. Further research is required in this area. Additional research is necessary to further delineate these underlying mechanisms.

\section{REFERENCES}

1. Gray L, Miller L W, Philipp B L, Blass E M. Breastfeeding is analgesic in healthy newborns. Pediatrics 2002;109:590-3.

2. Carbajal R, Veerapen S, Couderc S, Jugie M, Ville Y. Analgesic effect of breast feeding in term neonates: Randomised controlled trial. BMJ 2003;326:13.

\subsection{3}

\section{UNDERLYING MECHANISMS OF MATERNAL COMFORT}

\section{E Blass}

Department of Psychology, University of Massachusetts, Amherst, Massachusetts, USA

Taste

Taste and flavours effectively alleviate distress and reduce or block pain in preterms and neonates. They include sucrose, glucose, fat and milk. Animal studies have demonstrated that pain blockade is at the level of the spinal cord.

Taste- and flavour-induced analgesia's are opioid-mediated, although the exact mechanisms have not been sufficiently documented. Opioid mediation is confined to taste and flavour. It does not extend to contact or nonnutritive suckling-induced analgesia's. This may also be true in humans because infants born to women who used methadone during pregnancy, were not quieted by sucrose, but were when sucking a pacifier or through holding. The comforting system undergoes a transformation such that by 1 month infants have to be in eye contact with the person delivering the fluid for crying to be alleviated. By 3 mo, sucrose is not effective, either against crying or pain.

Underlying Mechanisms of Maternal Comfort

The system is extremely well conserved phylogenetically because all of the findings obtained in rat studies have been replicated in detail in human studies. One can conceptualize two interacting dynamics. One is the need to conserve energy. Direct metabolism studies have demonstrated that crying reduction causes a $13 \%$ savings in energy lost. This is likely garnered by all tissues, the brain especially. The other factor is cognitive/behavioural. In time, pain and discomfort must be recognized and appropriate, specific behaviours must be recruited to either avoid or escape them. This may help account for the diminution of sweet taste as an analgesic.

\section{WORKSHOP 2C - THE CONUNDRUM OF PCA BY PROXY}

Chair: Steven Weisman

\subsection{3}

THE CONUNDRUM OF PCA BY PROXY

$\underline{M}$ Czarnecki $^{1}, \underline{S \text { Weisman }}^{1,2},{\underline{C} \text { Berde }^{3}}^{3}$

${ }^{1}$ Children's Hospital of Wisconsin; ${ }^{2}$ Medical College of Wisconsin,

Milwaukee, Wisconsin; ${ }^{3}$ Children's Hospital and Harvard Medical School, Boston, Massachusetts, USA

Pro:

Children in pain are entitled to the safest, most effective method of pain control possible. Conventional patient-controlled analgesia (PCA) allows patients to manage pain by administering small doses of an intravenous opioid, with or without a background (basal) infusion. Patient Controlled Analgesia (PCA) has been shown to be safe and effective for managing pediatric pain, and superior when compared to other methods of parenteral analgesic administration. Children of any age or developmental level should have access to this method of pain control, if at all possible. PCA by proxy or parent-nurse-controlled analgesia allows a parent or nurse to titrate small doses of opioids safely and in a timely manner. Many of the advantages (timely, effective pain management, the ability to premedicate prior to activities) afforded to the conventional (older and developmentally normal) PCA users can be reaped by the recipients of PCA by proxy. With proper dosing, education, and monitoring, PCA by proxy can be used in nearly all children unable to use a conventional PCA. Support will be shared by reviewing current literature, as well as results of a retrospective study recently conducted at $\mathrm{CHW}$.

Con:

In December of 2004, the Joint Commission for the Accreditation of Hospitals Organization (JCAHO) issued a sentinel event alert cautioning health care providers PCA by proxy. Specifically, JCAHO stated that "infants and small children are not appropriate candidates" for PCA by proxy. While there are advantages to parents or nurses being in charge of a PCA for those children who are unable to operate it themselves, there are certainly risks as well.

Some clinicians take the middle position that PCA by proxy by nurses is relatively safe in most settings, but that PCA by proxy by parents should be restricted to opioid-tolerant patients in palliative care.

Dr Berde will discuss the risks of PCA by proxy, and, for discussion purposes, will take the position that PCA by proxy should be used only in relatively limited circumstances. 
WORKSHOP 2D - ADULT INFLUENCES ON

CHILDREN'S PAIN AND DISTRESS: EXPANDING THE PARADIGM

Chair: Ronald Blount

\subsection{4}

ADULT INFLUENCES ON CHILDREN'S PAIN AND DISTRESS: EXPANDING THE PARADIGM

R Blount ${ }^{1}$, S Williams ${ }^{2}$, L Walker ${ }^{3}, \underline{Z ~ K a i n}^{4}$, A Caldwell-Andrews ${ }^{4}$, M Weinberg ${ }^{4}$, L Mayes ${ }^{4}$, K Devine ${ }^{1}$, P Cheng $^{5}$, L Seri ${ }^{1}$

${ }^{1}$ University of Georgia, Athens, Georgia; ${ }^{2}$ Vanderbilt University; ${ }^{3}$ Vanderbilt University Medical Center, Nashville, Tennessee; ${ }^{4}$ Yale University School of Medicine, New Haven, Connecticut; ${ }^{5}$ Duke University Medical Center, Durham, North Carolina, USA

Parents and medical staff can have beneficial or detrimental effects on children during acute painful medical treatments. Adults promote children's coping by distracting or coaching them to use coping behaviors. Conversely, reassurance, apologies, empathy, criticism, and giving control to children seem to increase child distress and pain. These assessment findings have yielded empirically derived treatment protocols.

This symposium demonstrates significant extensions of the adult-child behavioral interaction paradigm to other pediatric populations and medical procedures. Researchers in the areas of functional gastrointestinal disorders (FGID), anesthesiology, and acute procedural pain provide findings from their laboratories.

The goals of this workshop are fourfold:

1. Participants will learn how parental behavior might increase or decrease chronic/episodic pain using the example of children who experience FGID.

2. An example of evidence-based practice will demonstrate how parental behavior may be utilized to decrease children's distress before anesthesia induction.

3. Parent-staff-infant behavioral interactions during immunizations will reveal specific coping-promoting and probable distress-promoting adult behaviors.

4. By providing multiple examples of the potent influence of parent-child behavioral interactions on children's pain and coping, participants will be encouraged and better equipped to utilize this model in their own pediatric settings.

There will be opportunities for questions and discussion.

\subsection{1}

\section{THE INFLUENCE OF PARENTAL ATTENTION VERSUS} DISTRACTION ON CHILDREN'S SYMPTOM COMPLAINTS

\section{S Williams, $\underline{L}$ Walker}

Division of Adolescent Medicine and Behavioral Science,

Vanderbilt University Medical Center, Nashville, Tennessee, USA

Solicitous behaviour by significant others may reinforce symptoms and disability associated with illness. In the pediatric literature, children with functional gastrointestinal disorders (FGIDs) have been shown to perceive more solicitous parent behaviour. However, observational studies of the impact of parents' behaviour on children's pain would provide a stronger test of this social learning principle. This study used a water load symptom provocation task (WL-SPT) to manipulate abdominal discomfort in the laboratory to assess the impact of parent attention versus distraction on children's symptom complaints.

Participants were patients with FGID pain and healthy children ages 8-16 years. Mild abdominal discomfort was induced using the WL-SPT (drinking water until full). Parents were trained to Attend (focusing on body sensations, reassurance) or Distract (talk about something else), or received No Training. Parents interacted with their children for 5 minutes following WL-SPT. Conversations were coded for the frequency of children's verbal symptom complaints. Self-report GI symptoms were obtained before and after the interaction.

Results indicated that FGID patients made more complaints than well children, more complaints were made in the Attention than Distraction condition, and females made more complaints than males. Female pain patients in the Attention condition made the most symptom complaints. The same pattern of results was found for self-reported GI symptoms. In summary, attentive and solicitous parent behaviour amplified the distress experienced by children with recurrent pain, and especially so for females. Conversely, distraction successfully reduced pain patients' and well children's symptom complaints. These results have implications for designing interventions to decrease discomfort associated with FGIDs. Acknowledgements: Supported by R01 HD23264.

\subsection{2 BEHAVIORAL TRAINING PROGRAM SIGNIFICANTLY DECREASES CHILDREN'S PREOPERATIVE ANXIETY}

\section{$\underline{Z}$ Kain, A Caldwell-Andrews, M Weinberg, L Mayes, R Blount}

Controversy exists regarding whether parents' presence during induction of anesthesia (PPIA) can effectively calm children. To date, the PPIA literature has focused primarily on parents' presence or absence rather than on what parents do in the operating room. We developed a training program based in part on findings from CAMPIS-related literature suggesting that certain types of parent behaviors are more calming than others.

Before surgery, children were randomized to standard of care, premedication (midazolam 20-40 minutes before surgery) or intervention conditions. Intervention consisted of an informational video, parent guided exposure/shaping exercises, coached distraction, supportive telephone contact, and a short preparation before surgery.

Children's state anxiety (mYPAS) was measured in the holding area, entrance to operating room, and upon introduction of anesthesia mask. Parents reported their own anxiety (STAI). Children were videotaped for later coding using the P-CAMPIS, a modification of the CAMPIS for the perioperative environment.

Group differences were found in children's anxiety over time: At Holding Area: Children's state anxiety was lower in the intervention group than in the premedication or control groups, and premedication and control groups showed similar anxiety levels; At Operating Room Entrance and Mask Introduction: The intervention and premedication groups had similar levels of anxiety; both lower than the control group. Parents' state anxiety was lower in the intervention group. Finally, P-CAMPIS analysis indicated there were different behaviors exhibited by parents in the intervention versus the other groups.

This behavioral treatment lowered children's anxiety as successfully as premedication during induction of anesthesia and also enabled children to arrive at the hospital less anxious.

\subsection{3}

\section{THE IMPACT OF ADULT BEHAVIOURS AND VOCALIZATIONS ON INFANT DISTRESS DURING IMMUNIZATION}

\section{K Devine, R Blount, P Cheng, L Seri, L Simons}

\section{University of Georgia, Athens, Georgia, USA}

This study examined the impact of parents' and nurses' behaviours on infant distress following immunization injections. Forty-eight infants (aged 2-15 months) received immunization injections at a health department. The occurrence of specific adult and infant behaviours and vocalizations were coded in five-second intervals using the Child-Adult Medical Procedure Interaction Scale-Infant Version (CAMPIS-IV), a scale designed specifically for use with infants and accompanying adults. Similar to the results from studies with children, adult behaviours that focused the infant's attention away from distress, such as distracting with an object/toy and talking to the child about nonprocedural topics, were associated with lower levels of crying. Other behaviours that are typically classified as soothing, such as holding the infant facing inward against the parent's chest and the infant sucking on a pacifier or bottle, were also associated with lower levels of cry. Consistent with findings from observational studies with preschoolers, reassuring, empathizing, and apologizing to the infant were not associated with lower levels of distress. In fact, these behaviours tended to be associated with higher levels of distress.

Sequential analyses were conducted to examine the level of cry that 
preceded specific adult behaviours and the impact of those behaviours on subsequent cry levels. A pattern of results similar to the first set of analyses was obtained. Together, these results suggest specific adult behaviours that may reduce infant distress following painful injections.

\section{PLENARY 3 - OFF-LABEL PRESCRIPTIONS FOR INFANTS AND CHILDREN}

\subsection{1 CHILDHOOD ANALGESICS: GETTING OFF-LABEL DRUGS ON LABEL}

KJS Anand

Pediatrics, Anesthesiology, Pharmacology \& Neurobiology, UAMS

College of Medicine; Pain Neurobiology Laboratory, Arkansas

Children's Hospital Research Institute, Little Rock, Arkansas, USA

BACKGROUND: Historically, most drugs have not been tested in infants or children before release for marketing. Recent developments in pediatric pharmacology suggest that adult drug testing data do not apply to pediatric patients.

PURPOSE: This talk will summarize initiatives of the US Food and Drug Administration (FDA) and other national agencies to enhance the study of newer (on patent) and older therapeutic agents (off patent) in pediatric age groups.

METHODS: Official documents issued by the FDA, NIH, CIHR and other agencies, summaries of legislative acts passed by the US Congress, expert testimonies in the public domain, and published statements from multidisciplinary expert focus groups were reviewed.

RESULTS: Specific procedures for obtaining drug labelling information have been developed that apply to on-patent and off-patent drugs. Regulatory agencies mandate certain elements of study design to use the results of clinical investigation for changing the drug label. At present, many analgesics have not been approved for the pediatric indications in their current and routine usage. Few analgesics are listed among the high priority list of drugs developed and published annually by the FDA. Research funding may be available for studying off-patent drugs whereas active collaborations between academia and the pharmaceutical industry will fund the study of on-patent analgesic agents for newborns, infants and children.

CONCLUSIONS: Most drugs used for analgesia in pediatric patients still lack dosing, efficacy, and safety studies. National agencies in the US and other countries are developing programs to close the gap between labelling data and routine clinical usage. Pediatric pain specialists can help to prioritize drug studies and develop strategies to overcome the obstacles for conducting drug trials in children.

\subsection{2 \\ PEDIATRIC ANALGESICS: WHY ADULT DATA IS INSUFFICIENT FOR REGULATORY AUTHORITIES \\ B] Anderson}

Anaesthesiology, University of Auckland, Auckland, New Zealand

BACKGROUND: Size, growth, maturation, ethical issues, disease spectrum and the potential for future harm are factors distinguishing pediatric from adult patients. These factors render the use of adult pharmacology data unsuitable for extrapolation to children.

PURPOSE: This talk will summarize pediatric pharmacokinetic/pharmacodynamic differences and their importance for labeling new and old analgesic drugs.

METHODS: The variability in drug responses among individuals determines the limits of safety and efficacy for its clinical use. Population modeling methods provide advantages for paediatric studies because they can be used to analyse sparse data, sampling times are not crucial (can be fitted around clinical procedures) and individuals with missing data may still be included. Explanatory covariates in these models may explain the predictable part of the between-individual variability.

RESULTS: Growth and development can be investigated using size and age as covariates. Problems attributable to co-linearity can be approached using size as the first covariate. Size standardisation is achieved using allometric scaling, a mechanistic approach that has a strong theoretical and empirical basis. Age is used to describe maturation of clearance. The quantitative models used to describe the maturation of pharmacokinetic processes (clearance, volume, absorption, bioavailability) vary depending on the span of the ages under investigation. Measures of response are not always straight-forward, vary with age and can be more difficult to quantify in children. Explanatory variables may include receptor type, number and function. Formulations continue not to be child friendly, creating difficulties in administration and reducing drug effect. Simulation using parameter estimates and their variability can be used to investigate large numbers of children, many more than is possible using real children for a fraction of the cost; an advantage for clinical trial development.

CONCLUSIONS: Mainly as a result of government driven incentives, pediatric population modeling has expanded greatly in the past decade and is used routinely during drug development and investigation. Clinical trials of analgesic drugs in children will continue to benefit from the use of these methods. Mandated needs for pediatric data by the FDA will inform their clinical use and thereby improve analgesic safety and efficacy.

PLENARY 4 - LONG-TERM CONSEQUENCES

\subsection{1 COMPLEX LONG-TERM EFFECTS OF INFANT PAIN R Eckstein Grunau}

Department of Pediatrics, University of British Columbia, Michael Smith Foundation for Health Research, Vancouver, British Columbia

Medically-related pain in infancy has a range of effects, depending on multiple factors including the environmental context of the child. In infants born very preterm, repeated pain during hospitalization in the neonatal intensive care unit (NICU) is an increasing concern since recent data suggest effects are more widespread than changes to pain systems. Infants born very preterm are vulnerable to neurodevelopmental problems in attention, executive function, cognition and behaviour, leading to a substantial burden of suffering in school and family life. Little is known about the etiology and mechanisms leading to these problems, however, multiple lines of evidence implicates early pain-related stress as one potential contributing factor. Due to the immaturity of physiological and behavioural regulatory systems, and vulnerability of the developing brain, extended or repeated pain in infants born very preterm likely have more widespread effects not seen in more mature children. In human preterm infants, invasive procedures have spin-off effects whereby the infant becomes hypersentive to a continuum of standard nursing caregiving, in addition to skin-breaking events normally associated with pain, leading to high "allostatic load" of overall stress. Repeated activation of nociceptive and tactile systems during neonatal intensive care may directly or indirectly impact the developing hypothalamic-pituitary-adrenal-axis, and the developing brain. There is recent evidence that cortisol levels are high in infants born extremely preterm, months after NICU discharge, which has implications for long-term cognition, learning, memory and health. Objectives are to discuss relationships of early pain in the NICU and/or surgery on later pain sensitivity, to address evidence for altered stress systems (cortisol, behaviour) in former preterm infants and children in relation to neonatal pain, to describe potential for impact on the brain, and to examine the extent to which environmental factors, such as mother stress and interactive behaviours, may ameliorate effects of neonatal pain.

\subsection{2}

\section{CHILDHOOD PAIN GROWN UP}

M Hotopf

Institute of Psychiatry, King's College London, London, United Kigdom

There are relatively few long term follow up studies of children with chronic pain. This presentation will review some of these, focussing particularly on two British birth cohorts, the Medical Research Council 
National Survey of Health and Development (1946) and the National Child Development Study (1958). Data from these studies indicates that children with persistent unexplained abdominal pain and headaches go on to experience more physical symptoms in adult life, and more anxiety and depression, than do healthy children. Further, the available data suggests that children with chronic pain problems experience worse health outcomes than many children with better defined severe physical diseases. The implications of these findings and the relationship between childhood pain symptoms and adult medically unexplained symptoms and syndromes will be discussed.

\section{WORKSHOP $3 \mathrm{~A}$ - SCHOOL AVOIDANCE AMONG COMPLEX PEDIATRIC CHRONIC PAIN PATIENTS: IDENTIFICATION AND MULTISYSTEMIC TREATMENT \\ Chair: Renee Ladwig}

\subsection{1}

\section{SCHOOL AVOIDANCE AMONG COMPLEX PEDIATRIC CHRONIC PAIN PATIENTS: IDENTIFICATION AND MULTISYSTEMIC TREATMENT}

$\underline{\text { K Khan }}^{1}$; $\underline{\text { Ladwig }}^{2}$; $\underline{\text { S Weisman }}{ }^{1}$

${ }^{1}$ Medical College of Wisconsin/Children's Hospital of Wisconsin;

${ }^{2}$ Children's Hospital of Wisconsin, Milwaukee, Wisconsin, USA

The goal of this workshop is to provide an overview of the interface between chronic pain and school avoidance, and intervention options that flow from an explanatory model. School avoidance can dramatically complicate the medical presentation of patients seeking treatment for chronic pain, as symptom severity is often greater than would be expected, which presents a challenge to providers. School avoidance dynamics can often play a primary role in the development and exacerbation of pain symptoms, level of functional disability, and response to treatment. Review of clinical material from our Chronic Pain Centre indicates that $38 \%$ of cases were classified by consensus as meeting school avoidance criteria. Comparing the school avoidance families to other families seen in the clinic, it is clear that there are numerous and systematic differences between the groups. Given the concerning maladaptive outcomes associated with school avoidance behaviour, and the documented high rate of school absenteeism within the chronic pain population, it is imperative that children prone to school avoidance behaviour be identified and that empirically validated treatments are available. It is our experience that addressing school avoidance dramatically impacts the clinical presentation and treatment outcomes of chronic pain problems. This workshop will provide clinicians with an understanding of how to assess the unique variables and vulnerabilities of patients presenting with chronic pain and school avoidance. Our clinical experience indicates that key factors are present and can be used to identify the presence of school avoidance behaviours. Cognitive behavioural interventions within a collaborative family health model will be presented as the preferred treatment modality. Specific case examples will be used to illustrate these assessment and intervention strategies.

\section{WORKSHOP 3B - RELAXATION, IMAGERY AND VIRTUAL REALITY TECHNIQUES TO CONTROL PROCEDURAL PAIN AND FEAR Chair: Bernie Whitaker}

\subsection{1 \\ VIRTUAL REALITY AND ANIMATED MOVIE DISTRACTION FOR PROCEDURAL PAIN}

\section{BS Lange}

University of South Australia, Adelaide, Australia

Distraction techniques aim to draw attention away from thoughts associated with medical procedures and focus the limited available capacity for attention to a nonprocedural-related task. Distraction techniques are available in many forms, for example, blowing bubbles, conversation, books, movies and computer games. Typically the techniques range from passive to active interventions. Virtual Reality (VR) is considered to be an active technique. It consists of a head mounted display through which a virtual environment / reality can be viewed and interacted with by the child. Virtual reality distraction was first investigated in 2000 by Hoffman and colleagues at the University of Washington. The immersive and interactive features of VR are the basis upon which the tool is thought to be an effective distraction technique. Virtual Reality distraction has been found to be effective in children undergoing procedures that frequently cause pain and distress such as dressing changes, physiotherapy for burns and postoperative physical therapy. Results are, however, mixed in larger samples of children undergoing more routine medical procedures such as venipuncture. This presentation will demonstrate the features of VR systems and discuss findings of previous research involving VR distraction for pediatric medical procedures. The clinical application and practical aspects of VR distraction will be discussed and the findings of a study comparing the use of VR with watching an animated movie in children undergoing venipuncture and wound care procedures will be presented.

\subsection{2}

\section{COGNITIVE BEHAVIOUR THERAPY IN THE MANAGEMENT OF PROCEDURE-RELATED PEDIATRIC PAIN}

\section{Lioss:}

School of Psychology, University of Southampton, United Kingdom

Cognitive-behaviour therapy (CBT) is an evidence-based intervention in the management of procedure-related pain and distress in children. This talk will first explore the fundamental principles underlying its use, then research studies and particularly randomized controlled trials that have evaluated the approach will be reviewed and finally there will be a description of the techniques that are widely used in actual clinical practice. The use of CBT in pediatric pain management will be contextualized in the light of developmental personality and coping theories.

\subsection{3}

\section{RECONSTRUING PROCEDURAL FEAR AND PAIN REALITIES: FROM DISTRACTOR TO DISTRACTION AND BEYOND}

\section{BH Whitaker}

\section{University of Ballarat, Victoria, Australia}

Distraction has been used informally for centuries to help children cope with pain. Increasingly, over the past 25 years, health professionals have employed a variety of distractors with varying efficacy in both research and practice to reduce fear and pain in children. However, what is it about a particular distractor that elevates it to the level of effective distraction? This presentation will explore the concept of distraction from the child's perspective by drawing upon a Personal Construct (Kelly, 1955) view of how the child construes his or her reality. The notion of a distractor versus distraction will be discussed before moving on to the realm of more complex reality reconstructions that can be achieved through imagery. Conceptually, between distraction and imagery lies relaxation, a technique that is useful in helping a child gain control over procedural fear. Indeed, sometimes in the heat of the moment when best attempts at distraction or imagery prove to be ineffective, simply resorting to focused deep breathing can help the distressed child regain a sense of control. In this presentation, delegates will have an opportunity to engage in a simple deep breathing and progressive muscle relaxation technique that can be used with children who experience procedural fear and pain. The presentation will conclude with a short video illustrating the plasticity of the procedural pain reality when a child undergoing venepuncture is given an opportunity, through imagery, to engage in his or her own reality reconstruction.

\section{REFERENCES}

1. Kelly GA (1955). The psychology of personal constructs. New York: Norton. 


WORKSHOP 3C - PERCEPTUAL SENSITIVITY
AND AVOIDANCE OF SENSATION AS THE
FOCUS OF TREATMENT IN PEDIATRIC
CHRONIC PAIN - TOWARDS A NEW
APPROACH TO TREATMENT IN CHRONIC
DEBILITATING PAIN
Chair: Gunnar Olsson

\subsection{1}

AFFECT REGULATION AND PAIN SENSITIVITY: THE INTERACTION OF MATERNAL AND CHILD EMOTIONS $\underline{\text { S Franks }}$

Department of Pediatric Anaesthesia and Pain Management, Royal Children's Hospital, Victoria, Australia

BACKGROUND: Research in pediatric chronic pain outlines a multitude of factors that can contribute to distress and disability associated with pain. However there is a paucity of research investigating emotional regulation and pain sensitivity in young people with persistent pain.

PURPOSE: To investigate the (1) influence of maternal emotional regulation on children's anxiety, depression, somatization and function, (2) interaction between maternal emotional distress and children's painsensitive temperament and emotional regulation, and how these factors influence children's anxiety, depression, somatization and function.

METHODS: Sixty-two children and adolescents referred to the Children's Pain Management Clinic at the Royal Children's Hospital and their mothers participated in the study. Mothers were interviewed separately using the semi-structured Meta-Emotion Interview (1999) regarding their awareness, acceptance, and emotional regulation of their own and their child's emotions, specifically, sadness, anger, and fear. In addition, mothers and children completed self-report questionnaires. Maternal emotional distress was assessed using a composite variable defined as the sum of the mean of scores on the Pediatric Inventory for Parents, Family Emotional Involvement and Criticism Scale, and Interpersonal Reactivity Index. Path modeling, which enables the estimation of relationships between observed variables, was used to test the proposed relationships. Ackowledgements: Dr George Chalkiadis, Royal Children's Hospital; Dr Marie Joyce, Australian Catholic University.

\subsection{2}

"THIRD WAVE" BEHAVIOURAL AND COGNITIVE THERAPIES FOR CHRONIC PAIN: BRIDGING PHILOSOPHICAL AND DEVELOPMENTAL DIVIDES

\section{LM McCracken}

Royal National Hospital for Rheumatic Diseases; University of Bath, Bath, United Kingdom

Cognitive and behavioural treatment approaches outside of pain management are evolving. This evolution involves new understandings of cognition, human suffering, and behaviour change, and an emphasis on clinical methods incorporating processes such as acceptance, mindfulness, values, and self. As these approaches are designed for multiproblem patients who show significant avoidance, have a history of treatment failure, and may demonstrate social complexities, they appear to be particularly applicable to complexly disabled chronic pain sufferers. The general developments within behavioural and cognitive therapies are referred to as "third wave" to note their place as an extension of earlier operant and cognitivebehavioural approaches. Within chronic pain management they have been recently referred to as contextual cognitive behavioural therapy (CCBT) to emphasize their incorporation of a pragmatic, functional, and nonmechanistic model of behaviour. As is often the case, applications of these innovative methods for adults have led the way of their applications for young people. During the workshop Dr McCracken will present the model and methods of CCBT in relation to currently established cognitive behavioural approaches, review the state of evidence of "third wave" approaches, and discuss the most recent research and clinical develop- ments of CCBT for young people. These include the development of assessment instrument development and modification of such methods as mindfulness training.

\subsection{3 \\ EXPOSURE AND ACCEPTANCE IN THE REHABILITATION OF CHILDREN AND ADOLESCENTS WITH CHRONIC DEBILITATING PAIN SYNDROMES}

RK Wicksell, GL Olsson

Pain Treatment Service, Astrid Lindgren Children's Hospital,

Karolinska University Hospital, Stockholm; Department of

Psychology, Uppsala University, Uppsala, Sweden

In developing a rehabilitation program for young people with chronic pain, it was hypothesized that avoidance of pain-related stimuli is central to disability and generates an increase in the patient's vulnerability to pain-related stimuli. Therefore, cognitive behaviour therapy (CBT), and particularly exposure, was considered the core intervention. Recent developments within CBT, such as Acceptance and Commitment Therapy (ACT), suggest an approach that, rather than focusing on alleviation of pain and distress by using control-oriented techniques, promotes acceptance of negative private events as a mean to facilitate exposure toward activities that are meaningful although possibly painful. To date, conceptual and empirical advances in acceptance-based behavioural interventions such as ACT have shown promising results in a broad range of clinical disorders, including chronic pain. This contextual form of behaviour therapy seems particularly promising for patients presenting with therapy resistant conditions, such as chronic pain, for which untreated symptoms represent major barriers to an active and meaningful life. In a randomized control trial, we compared an exposure-based behaviour therapy emphasizing acceptance to a multidisciplinary approach including amitriptyline for children and adolescents with chronic idiopathic pain. Preliminary results show significant differences with regards to functional disability, quality of life and pain in favour of the ACT approach. Data from this and from two different pilot studies will be presented, discussing relevant findings as well as clinical difficulties.

\begin{tabular}{c}
\hline WORKSHOP 3D - MIXED METHODS AT \\
WORK: CHALLENGES AND OPPORTUNITIES \\
IN AN INTEGRATIVE QUANTITATIVE- \\
QUALITATIVE STUDY OF PEDIATRIC PAIN \\
Chair: Lonnie Zeltzer
\end{tabular}

\subsection{1}

CASE STUDY 1: A POSTERIORI INDEPENDENT ANALYSES: DISCREPANCIES BETWEEN PARENTS' RATING OF DAUGHTERS' SOMATIC SYMPTOMS AND USUAL PAIN VERSUS PARENTS' RATING OF SONS' SOMATIC SYMPTOMS AND USUAL PAIN

I Clemente, J Tsao, M Meldrum, L Zeltzer

UCLA Center for the Study and Treatment of Pain, Pediatric Pain Research Program, Department of Pediatrics, David Geffen School of Medicine at UCLA, Los Angeles, California, USA

This is the first of three case studies, emerging from a mixed-methods project investigating the experience of children with recurrent pain and the biopsychosocial pain treatment model used at the UCLA Pediatric Pain Clinic. These three case studies present methods and challenges of integrating the project's three components: a quantitative study utilizing self-reported written measures; an oral history project employing videotaped in-depth semi-structured interviews with parents and patients; and a sociological study using videotaped naturally occurring interactions between clinicians, parents, and patients.

A first quantitative study examined sex differences in parent and child ratings of the children's somatic symp- 
toms and usual pain. We found sex differences in the relationship between parent ratings of a broad range of somatic symptoms, including faintness, gastrointestinal problems, headaches, and chest pain, and children's own ratings. Parent ratings of their daughters' somatic symptoms were significantly correlated with daughters' own somatic symptom $(r(82)=0.66, P<0.001)$ and usual pain ratings $(r(84)=0.23, P>0.05)$. For boys, parent ratings of their sons' somatic symptoms were significantly correlated with sons' own somatic symptom ratings $(r(31)=0.87, P<0.001)$. However, parent ratings of sons' usual pain were not correlated with sons' usual pain ratings $(r(33)=0.28$, NS). In our qualitative analysis of families' answers to clinicians' questions, we found that, whereas parents and daughters verbally disagreed while conveying information to the clinicians, sons often remained silent, letting their parents talk and contributing little to symptom presentation. Silence may not be an indication of greater attunement between parents and sons, but of deeper disagreement. Sons may feel they will not be heard or understood, while daughters feel more able to express their personal experiences of pain and somatic symptoms. Additional data from separate oral history interviews with parents and patients will help elucidate this discrepancy.

\subsection{2 \\ CASE STUDY 2: FROM QUANTITATIVE FINDINGS TO QUALITATIVE ANSWERS: TREATMENT EXPECTATIONS IN PEDIATRIC CHRONIC PAIN PATIENTS AND THEIR PARENTS}

L Tsao, M Nutkiewicz, L Zeltzer

UCLA Pediatric Pain Program, Department of Pediatrics, David Geffen School of Medicine at UCLA, Los Angeles, California, USA

Patient expectations regarding treatment, particularly for complementary and alternative medicine (CAM) interventions, have important implications for treatment adherence, attrition and clinical outcome. There has been little systematic research regarding treatment expectations for CAM and conventional medicine approaches for pediatric chronic pain problems. Quantitative methods were initially used to examine ratings of the expected benefits of CAM (ie, hypnosis, massage, acupuncture, yoga, and relaxation) and conventional medicine (ie, medications, surgery) interventions in 45 children ( 32 girls; mean age $=13.8$ years \pm 2.5$)$ and parents (39 mothers) presenting for treatment at a specialty clinic for chronic pediatric pain. Respondents rated the expected benefit of each approach on a $1-5$ Likert scale: $1=$ not at all (helpful), $2=$ a little, $3=$ some, $4=$ a lot, $5=$ completely (helpful). Notably, children rated the overall expected benefits of CAM and conventional treatments to be fairly low $(\mathrm{M}=2.2 \pm 0.6)$ with parents' expectations only somewhat more positive $(M=2.6 \pm 0.7)$. Qualitative methods were then used to shed light on these quantitative findings. Responses to questions about patient-doctor communication, collected from oral history interviews of the children prior to their first clinic appointment, were examined. Textual analysis revealed that children reported initial confidence in doctors' ability to help, but that, over time, they sensed that physicians were using trial-and-error, "approaches" to treat their pain. The children concluded that doctors have no special insights into, or efficacious remedies for, their pain. These qualitative findings suggested that children's disillusionment with physicians' unsystematic approaches to chronic pain were a salient factor in lowered treatment expectations. Thus, a probe of children's experiences via qualitative methods, revealed possible explanations for the results of the quantitative study. Additional examples are discussed.
4.205.3

CASE STUDY 3: FROM QUALITATIVE FINDINGS TO POTENTIALLY GENERALIZED FINDINGS: FROM THE "HOW?" TO THE “HOW MANY AND HOW OFTEN?"

M Nutkiewicz ${ }^{1}$, J Tsao ${ }^{2}$, M Meldrum

${ }^{1}$ Program for Torture Victims; ${ }^{2}$ UCLA Pediatric Pain Program, Department of Pediatrics, David Geffen School of Medicine at UCLA, Los Angeles, California, USA

In our sample of children with chronic pain ( $\mathrm{n}=114,81$ female), parent report of children's functioning, as measured by the Child Health Questionnaire was significantly worse than in a sample of healthy children $(\mathrm{n}=225 ; 111 \mathrm{female})$. Thus, children with chronic pain had significantly worse scores for self-esteem $(\mathrm{t}(337)=11.09)$, well-being $(\mathrm{t}(337)=12.69)$, as well as overall physical $(\mathrm{t}(337)=23.83)$ and psychosocial functioning $(t(337)=3.89)$ (all $\left.P^{\prime}<<0.001\right)$. This finding was not surprising in the light of earlier studies, but was not fully explained by the quantitative scores. Such feelings of psychosocial distress could have multiple etiologies, and could be the results of recurrent pain or pre-existing feelings which act to reinforce pain behaviour. A qualitative analysis of the oral history data revealed two consistent factors: a sense of isolation from "normal" children and a frustration with not having their pain taken seriously, both of which caused considerable distress for the children. These statements were characteristic: "No one knows what it's like for me." "They don't ask it, they just assume that I'm faking it. It bugs the hell out of me. I'm not going to try to get them to believe me. I don't need to. Whether people agree with me or not, it's actually happening, then it doesn't matter. If it's happening, it's happening." "She's over-exaggerating, she's milking it for all it's worth. It's really not true, and it really upsets me when I hear that, because I'm doing the best I can.” The significance of the children's isolation and frustration in contributing to psychosocial distress as possible targets for assessment and intervention are discussed.

\section{WORKSHOP 3E - COPING AND CHILDREN'S PAIN: FUTURE DIRECTIONS FOR THEORY, RESEARCH AND PRACTICE Chair: Tiina Piira}

\subsection{1 \\ COPING AND CHILDREN'S PAIN: FUTURE DIRECTIONS FOR THEORY, RESEARCH AND PRACTICE \\ I Piira $^{1}$, R Blount ${ }^{2}$, L Goubert ${ }^{3}$ \\ ${ }^{1}$ Sydney Children's Hospital and University of New South Wales, Sydney, Australia; ${ }^{2}$ University of Georgia, Athens, Georgia, USA; ${ }^{3}$ Ghent University, Ghent, Belgium}

When faced with pain, children cope in the best way they can with the resources available to them. Coping strategies may be utilized to regulate emotions, cognitions, behaviour, physiology and the social and physical environment in response to a stressor such as pain. With increasing cognitive sophistication, children have access to a broader repertoire of coping strategies. Their coping efforts are motivated by the outcomes they wish to attain, which may differ from the outcomes that health professionals wish to attain. For example, a child may want to avoid or escape from the treatment room and may engage in coping behaviours motivated by this goal, whereas health professionals may be motivated to carry out a necessary medical procedure.

Researchers from 3 continents will highlight some of the key issues, challenges and advances in the field of coping and children's pain, as well as providing some of the latest research from their own labs.

This workshop will outline the evidence on the efficacy of methods to promote optimal coping in children across a variety of acute and chronic/recurrent pain contexts. The important role that parental coping has on children's pain outcomes will also be reviewed. Clinical and theoretical implications will be considered, with opportunities for questions and discussion. 
The primary aims of this workshop:

1. Participants will gain a better understanding about cognitivedevelopmental considerations in children's coping.

2. Examples of evidence-based, age-appropriate interventions to facilitate children's coping in acute and chronic pain contexts will be presented.

3. Participants will learn about a new measure of parental catastrophizing about their child's pain.

4. The role of parental catastrophizing and parental behaviour will be considered in terms of how this impacts on child pain outcomes.

5. Areas of promise in terms of advancing theory, research, and clinical practice in this field will be identified.

\subsection{1 \\ COGNITIVE-DEVELOPMENT AND CHILDREN'S COPING WITH PAIN}

T Piira

Sydney Children's Hospital and University of New South Wales, Sydney, Australia

From infancy, individuals are capable of some regulation of physiological arousal, behaviour and emotions. In infancy, coping responses are typically automatic responses, not mediated by conscious planning. However, with increasing cognitive sophistication, children have a greater range of coping responses available to them.

A child's coping is influenced by their cognitive and behavioural capacities for regulation of the self, as well as regulation of their physical and social environment. Coping efforts are mediated by the emergence of intentionality, representational thinking, language, metacognition, and the capacity for delay.

Piira will review the latest literature on the development of attentional processes and will apply this to the context of pain management, and specifically to the use of attention-based coping strategies such as distraction.

There will be a detailed presentation of recent experimental evidence, using the cold pressor pain task, concerning the conditions that affect the efficacy of specific coping strategies for children of different ages. Data will also be presented on how the efficacy of a distraction-based coping strategy is affected by the provision of preparatory sensory information.

\subsection{2}

\section{CHILDREN'S COPING WITH CHRONIC / RECURRENT PAIN}

\section{R Blount}

University of Georgia, Athens, Georgia, USA

Inflammatory bowel disease (IBD) includes a group of chronic diseases, the most common of which are ulcerative colitis and Crohn's disease. Adolescents with IBD share symptoms of abdominal pain, diarrhea, urgency to defecate, intestinal or rectal bleeding, and weight loss. They also report higher levels of psychological problems and lower self-esteem than healthy controls. In spite of these difficulties, there are few psychologically based intervention studies that have addressed their needs. A diathesis-stress conceptualization guided the design of this treatment investigation. Pain and disease symptoms were viewed as physical diatheses that could adversely influence adolescent and parental adjustment. Poorer adolescent and parental adjustment, likewise, were seen as potential stressors that could worsen the adolescents' pain and other symptoms.

The treatment consisted of a 10-week skills-based, cognitive behaviourally oriented, coping and communication skills intervention for improving psychological adjustment and decreasing pain. Parents and girls participated concurrently in separate groups and together. Training components consisted of problem-solving, cognitive coping skills, relaxation, pain management, parental limit-setting, and communication skills. In-session practice, feedback, and homework were used. A pre-post design with 5-month follow-up was incorporated.

Pre-post and pre-follow-up benefits were noted on the Abdominal Pain Index; Functional Disability Inventory; the Family, Social, and Communication subscales of the Coping Health Inventory for Parents; and on adolescents' reports of self-esteem and their sense of inadequacy on the Behavioural Assessment Scale for Children. This investigation supports the efficacy of the intervention. It is likely the treatment would prove

beneficial with other groups. A briefer intervention is being developed.

\subsection{3 \\ PARENTAL CATASTROPHIC THINKING ABOUT THEIR CHILD'S PAIN AND THE RELATIONSHIP WITH PARENTAL DISTRESS, PARENTING BEHAVIOUR AND CHILD DISABILITY}

\section{Goubert}

Ghent University, Belgium

Responding negatively toward pain as if it were a sign of an impending health catastrophe has been shown to be of significant importance in models developed to explain pain complaint and pain-related disability. Pain catastrophizing has helped explain adjustment to chronic pain in numerous adult studies, and has more recently been investigated with children and adolescents in chronic pain.

The study of catastrophic thinking about pain has, however, been studied from a predominantly intra-individual perspective, perhaps at the expense of an understanding of inter-individual and wider social influences. People do not behave in a social vacuum but are highly influenced by social factors. Parental factors are likely to be of particular importance in our understanding of childrens' pain and disability. In particular, we need to know more about the extent to which parents catastrophize about their child's pain, and the impact catastrophizing about one's child may have on child pain behaviour.

This presentation will discuss the development and validation of a recently developed measure of parental catastrophizing about their child's pain (PCS-P) in a sample of parents of school children $(n=205)$ and a sample of children with chronic pain $(n=107)$. The contribution of parental catastrophizing about their child's pain in explaining (a) parental distress, depression and anxiety, (b) parenting behaviour, and (c) child's disability will also be discussed.

\begin{tabular}{c}
\hline RAPID COMMUNICATION WORKSHOP 3A- \\
THE DEVELOPMENT AND IMPLEMENTATION \\
OF (EVIDENCE-BASED) GUIDELINES ON \\
PAIN IN CHILDREN \\
Chair: Richard van Lingen
\end{tabular}

\subsection{1}

\section{THE DEVELOPMENT AND IMPLEMENTATION OF (EVIDENCE BASED) GUIDELINES ON PAIN IN CHILDREN}

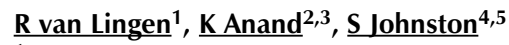

1Princess Amalia Department of Paediatrics, Division of Neonatology, Isala Clinics, Zwolle, Netherlands; ${ }^{2}$ Arkansas Children's Hospital; ${ }^{3}$ University of Arkansas for Medical Sciences, Little Rock, Arkansas, USA; ${ }^{4}$ The Children's Hospital at Westmead, Sydney, Australia; ${ }^{5}$ Groote Schuur and Red Cross Children's Hospitals, Cape Town, South Africa

EDUCATIONAL OBJECTIVES: Participants will obtain an understanding of which pitfalls have been found during the process of formulating the questions (patient and/or problem, intervention, comparison and (clinical) outcome), during the search for literature in Pubmed, Embase and other databases. Which studies can be used in developing the guidelines (level of evidence) and what has already been worked out and does not need a new search, but only updating. Participants will learn and discuss what are the guidelines that can be implemented and what kind of implementation offers the best results in keeping the guidelines up to date and effective in the daily practice, both in developed and developing countries.

BACKGROUND AND SUMMARY OF SESSION: In most countries a scarce amount of guidelines for children is available. Mostly individual hospitals or organizations like pain groups do have some sort of guidelines, but there is often no consensus. Evidence based guidelines are now emerging slowly, during the last few years, but although improvements in knowledge and attitudes have been made, there is little evidence that these 
guidelines result in improvements in clinical practice.

Funding for development of (evidence based) guidelines has been found from governments, companies or by other grants. In several countries committees and local research groups are in the process of developing and implementing the guidelines on pain assessment and therapy. This will be done both top down as bottom up. To avoid that workers in other countries have to do the same work all over again, the experiences, pitfalls and results in three different countries (in the United States, South Africa and the Netherlands) will be discussed.

The intended audience will be all participants working with children: nurses, nurse practitioners, psychologists and doctors.

\subsection{1}

\section{DUTCH EVIDENCE-BASED NATION WIDE GUIDELINES} FOR PAIN ASSESSMENT AND MANAGEMENT IN CHILDREN

\section{$\underline{R}$ van Lingen}

Princess Amalia Department of Paediatrics, Division of Neonatology, Isala Clinics, Zwolle, The Netherlands, on behalf of the Working Party for the development of guidelines for pain in children

In the past 3 years, representatives of pediatric, anesthesiological, surgical, psychological, pain and nursing societies have performed an EvidenceBased search to develop guidelines for pain assessment and management in children (0-18 years) in hospitals and outpatient clinics in the Netherlands. Primary questions in the domains of pain assessment, pain in mentally disabled children, (non-) pharmacological pain management, organization of management/assessment, and long-term consequences of pain were formulated. The literature was searched systematically for the different subsets and assessed for level of evidence and summarized in levels of conclusions, based on the analysis of the literature.

Searches of Medline (1980-2005), Cinahl, Psychinfo, The Cochrane Library, and references from review articles were performed, combining Mesh terms with free text words. Articles were identified in English, Dutch, German, French, Spanish and Italian.

For the different areas as assessment, pharmacological and nonpharmacological therapy in procedural pain, acute and chronic pain recommendations (guidelines, algorithms and dosing schemes) were made after counselling the soundboard group. In areas of pain management where evidence was weak, the committee gave expert opinion recommendations. In these area's of pain in children where no evidence-based data were found, we strive to stimulate research together with professionals of other pain societies abroad.

We have now started the implementation by publishing the results on the webs of the participating societies, by making a hard copy available, by organizing a national symposium and by local workshops in all hospitals for all those involved in the care of children.

Funding: Order of Dutch Medical Specialists

\subsection{2}

\section{EVIDENCE BASED GUIDELINES FOR MANAGEMENT OF} PAIN IN NEONATES: REPORT OF THE NIH/FDA NEONATAL PAIN CONTROL GROUP

\section{KAnand}

Recent advances in neurobiology and clinical medicine have established that preterm and term newborns experience acute, repetitive, and prolonged pain. Although analgesia is beneficial for their short term clinical stability and long term outcomes, pain control measures are adopted infrequently, because of unresolved scientific issues and old practice patterns. A Neonatal Pain Control Group, established by the FDA and NICHD as part of the Newborn Drug Development Initiative, addressed specific issues related to 1) management of pain associated with invasive procedures; 2) provision of sedation and analgesia during mechanical ventilation; and 3) mitigation of pain and stress responses during and after surgery in the newborn. Crosscutting themes addressed within each area of management included: 1) clinical trial designs; 2) drug prioritization; 3) ethical constraints; 4) gaps in our knowledge; and 5) future research needs. This speaker will summarize their discussions and deliberations, and pro- vide novel study designs for neonatal pain research.

\subsection{3 \\ EVIDENCE BASED GUIDELINES FOR CHILDREN: POSSIBILITIES IN A DEVELOPING COUNTRY}

\section{S Johnston}

The adoption of guidelines based on sound theory and evidence is the ideal we all strive towards. This may be more difficult to achieve in the $3 \mathrm{rd}$ world environment where we are constrained not only by financial and staffing limitations but also by the challenges of educating both our professional colleagues and the public.

This presentation will focus firstly on the different patient population groups prevalent in our developing society HIV, tuberculosis, malaria, burn injuries, trauma and interpersonal violence and the different problems they pose. South Africa is in the unfortunate position of having the largest HIV positive population in the world and thus far very little work has been done in the management of acute and chronic pain syndromes and associated illnesses in children with AIDS. Secondly it will look at the possible means of achieving better facilities and patient care, instituting appropriate pain management guidelines and distributing this knowledge to our medical colleagues.

\section{RAPID COMMUNICATION WORKSHOP 3B - THE PEDIATRIC ONCOLOGY PATIENT: FROM START TO FINISH \\ Chair: Kenneth Goldschneider}

\subsection{2}

\section{THE PEDIATRIC ONCOLOGY PATIENT: FROM START TO FINISH}

S Black, K Goldschneider, $\underline{M}$ Meyer, $\underline{N}$ Weidner

Division of Pain Management, Cincinnati Children's Hospital Medical Center, Cincinnati, Ohio, USA

Pediatric oncology care presents many opportunities for anesthesia pain services to provide a full spectrum of pain and symptom management. Some are obvious, such as providing patient-controlled analgesia and epidural analgesia to children undergoing surgery for tumour resection. Other activities, such as providing sedation for bone marrow biopsies and lumbar punctures may seem minor, but have significant impact upon the children and their parents. Despite improved outcomes for many pediatric malignancies, palliative care remains an essential component of pediatric oncology. Therefore, anesthesia pain services that care for oncology patients need to take the larger view of care. For instance, the "minor" procedures often require sedation or general anesthesia, can create scheduling issues for oncologists, families and pain/anesthesia personnel. Repeated trips to the operating room can generate fear in the children, and having a new physician each time prevents a feeling of continuity. Prolonged NPO times are disruptive to nutritional needs, and are unpleasant for the children. Anesthesia pain services that establish procedure rooms and recovery areas in or near to the oncology clinic can solve some of these issues, and thereby improve patient/family satisfaction. Reimbursement for the anesthetics and PACU care can be a positive revenue stream for anesthesia pain services that often "run in the red". The view of palliative care is changing; no longer a sign of "giving up," it is a discipline to be integrated into the overall patient care. This workshop approaches the pediatric oncology patient along a time continuum from diagnosis through treatment, palliation and death. We will discuss the integration of procedural and palliative pain care into the ongoing care map of the pediatric oncology patient, and discuss some of the issues in establishing these services. Topics will include establishing partnerships with the oncology service and hospital at large, family and patient needs, and pain and symptom management. 
RAPID COMMUNICATION WORKSHOP 3C FAMILY-CENTERED APPROACHES TO HELP PARENTS CARE FOR THEIR CHILD IN PAIN Chair: Beth Ely

\subsection{1 \\ IMPLEMENTATION OF A PARENT PAIN BROCHURE IN A CHILDREN'S HOSPITAL}

E Ely $^{1,2}$, E Kennedy ${ }^{2}$, H Lavella ${ }^{2}$

${ }^{1}$ Drexel University College of Medicine; ${ }^{2}$ St Christopher's Hospital for Children, Philadelphia, USA

We will discuss the creation and implementation of a parent-nurse partnership to improve pediatric pain management in an acute care pediatric hospital. Using a multidisciplinary approach, we created a parent pain brochure to distribute to all parents of hospitalized children. The brochure, written in both English and Spanish, highlighted pediatric pain assessment strategies, parent and health care provider responsibilities, and pain management interventions, both pharmacological and cognitive behavioural. Data on parent satisfaction with their child's pain management, nurses' knowledge of pediatric pain management and nurses' documentation of pain assessment were assessed using standardized tools. Focus groups were held with staff 8-9 months after the introduction of the brochure to evaluate both the process and brochure content.

Parent satisfaction as measured by a posthospital survey instrument showed improvement after the implementation of this educational effort. Overall scores for pain management the year previous to this effort were $69 \%$. Following implementation scores increased to $85 \%$ over a 6 -month period. In addition, outcome data includes knowledge gains for pediatric nursing staff and improved documentation and attention to pain problems for children hospitalized on this unit. Focus group data from 25 staff nurses in 6 groups indicated their satisfaction with the brochure content. More specifically, staff commented that having written materials provided them with the opportunity to discuss this content with patients and facilitated questions. Educational methods and strategies for continuing this effort over a 12 to 18 month period will also be presented.

\subsection{2}

\section{PARENTAL PAIN ASSESSMENT AND MANAGEMENT} PRACTICES AT HOME FOLLOWING AN INJURY

\section{R Yaffa Zisk}

Yale University School of Nursing and Yale University School of Medicine, New Haven, Connecticut, USA

PURPOSE: The majority of children's pain is assessed and managed by their parents, but there is meager literature regarding these practices. Parents are aware of their child's usual behaviour patterns and are thought to base their detection and management of the child's pain on changes in those patterns. Fractured limbs or clavicles due to accidental injury exemplify a common childhood pain experience where much of the pain assessment and management is carried out at home. Primarily this study examined the influence of parental perception of children's acute pain behaviours on acute pain management practices.

METHODS: This study employed a prospective design to explore parents' perception of their child's pain and management practices (pharmacological and nonpharmacological) at home following treatment in the emergency department for a fractured limb or clavicle. The participants included 50 parents / legal guardians of children aged 5-10 years. Analysis of the data included descriptive statistics, correlations, odds ratios, nonparametric tests, and regressions.

RESULTS: The results demonstrated that: parents provide their children with very few doses of analgesia, although most children experienced significant pain during the first two days; parents are attuned to many changes in their children's behaviour and base management decisions on them; while most children received analgesia based on exhibiting active, loud behaviours (eg, crying) ( $\mathrm{r}=0.63)$, children exhibited quiet behaviours more frequently than active ones ( $59.4 \%$ vs $31.2 \%$ ); and parents utilize many nonpharmacological pain alleviation methods which they report as being as helpful or more helpful than analgesia.

CONCLUSIONS AND IMPLICATIONS: The findings demonstrate that parents may still need additional assistance assessing and managing their children's pain at home. Especially for unplanned pain events, parents may benefit from interventions that assist them in recognizing cues children exhibit when in pain, and from comprehensive instructions in how to manage pain in an age-appropriate manner.

\subsection{3 \\ IMPLEMENTATION OF A PARENT-MEDIATED HOME PAIN MANAGEMENT PROTOCOL FOR YOUNG CHILDREN WITH SICKLE CELL DISEASE}

B Ely, N Mba, P O'Neal, C Coleman

Marian Anderson Comprehensive Sickle Cell Center at

St Christopher's Hospital for Children \& Drexel University College of Medicine, Philadelphia, Pennsylvania, USA

We will describe the implementation of a parent-mediated Home Pain Management Protocol (HPMP) for parents of young children with sickle cell disease (SCD). The HPMP consists of a series of 4 educational sessions (workbook format) developed for parents of young children (ages 5-8 yrs) with SCD. The sessions include information about causes of sickle pain, child pain coping strategies, sickle pain assessment, and pharmacological and cognitive behavioural and physical sickle pain management strategies. Age-appropriate learning activities for the children with SCD (ages 5-8 yrs) parallel parent content. Educational strategies used during the sessions are both didactic and interactive. Each session lasts for 60-90 minutes.

A pre-post-test design with parent/guardians acting as their own control is being used. Independent variables of child coping, parent assistance with coping, and parent knowledge are measured at the start of the sessions, directly after the 4 sessions are completed, and then at 6 months and 12 months after session completion. Eight mothers (23-48 yrs, M=34 yrs; all high school graduates, $50 \%$ with some college) of children primarily homozygous SS diagnosis $(87.5 \%)$ and male $(87.5 \%)$ have completed the protocol sessions with ongoing enrollment.

Data from the 8 mothers who have completed include a statistically significant improvement in knowledge of SCD $(\mathrm{P}<0.003)$ and a trend toward parent support of active pain coping skill development for their children. Subscale scores of the parent version of the Pain Coping Questionnaire (PCQ) pre- to postsessions indicate increased use of approach subscale items $(\mathrm{P}<0.014)$ such as information seeking. No significant differences were seen for the distraction and emotion-focused avoidance subscales. Process outcomes evaluated through the use of focus group interviews with parents held after the 4 sessions provide data yielded mothers' positive response to educational materials provided. Satisfaction with the session format and materials was also discussed.

Funding: Comprehensive Sickle Cell Grant P60-HL-70585

\begin{tabular}{c}
\hline RAPID COMMUNICATION WORKSHOP 3D - \\
HYPNOSIS IN CHILDREN \\
Chair: Lonnie Zeltzer
\end{tabular}

\subsection{1 \\ BASIC SCIENCE REGARDING HYPNOSIS AND ITS USE IN CHILDREN WITH CHRONIC PAIN}

C Wood

Pain Unit, Robert Debré Hospital, Paris, France

Recent neuroimaging studies have made us understand the importance of the modulation of the pain sensation by cognitive, affective and motivational processes.

Hypnosis-induced analgesia is an example of how cognitive processes may influence the pain sensation.

This aspect will be developed during the workshop as well as the use of different hypnotic techniques for children: 
- Conversational hypnosis: in many circumstances while in hospital, children are already in a hypnotic trance. Conversational techniques are fundamental to avoid anticipation of pain or in an extreme way, posttraumatic stress disorders.

- Visual imagery and Entonnox: using hypnotic techniques with Entonox, enables the child, during a procedure, to focalize on something else and go through the procedure in a better way

- Hypnotic techniques for certain pain syndromes: hypnosis has been used for phantom limb pain, back pains, headaches and many other symptoms. Examples will be given.

\subsection{2 \\ HYPNOSIS USED WITHIN HOSPITAL AND FAMILY SYSTEMS}

\section{$\underline{\text { L Kuttner }}$}

Pediatric Department, University of British Columbia, Vancouver, British Columbia

The language of hypnosis is often oriented towards change and future potentials. Children within families and in hospital for treatment are sensitive and highly responsive to how the information about impending procedures, pain and anxiety are framed and communicated. We will deconstruct traditional and limiting patterns of communicating that occur within these systems and provide alternative therapeutic hypnotic messages that allow children to feel empowered, and parents and hospital staff to feel more competent in treating and relieving children's pain and distress.

a) When a child is in pain and distress within a family, entrenched dysfunctional attitudes and behaviours interfere with positive coping. We will briefly explore the use of hypnotic reframing to facilitate change within that dynamic system.

b) Within a pediatric hospital a two-day training program that has been run in over 25 hospitals in North America and Europe using hypnosis to improve pain and procedural pain management will be discussed. This pain management seminar was designed to provide a model of care and create a consistent climate in which staff know the hypnotic techniques and language that promotes confidence in children and enables pain and anxiety to be well addressed and competently managed and relieved.

\subsection{3}

\section{HYPNOSIS: AN EVIDENCE-BASED INTERVENTION IN PEDIATRIC PROCEDURE-RELATED PAIN MANAGEMENT \\ C Liossi}

School of Psychology, University of Southampton, United Kingdom

Psychological approaches to symptom management are among the oldest and are an intrinsic part of medical practice in every culture. Suggestive therapy is probably the oldest of all therapeutic methods and hypnosis under various names has been used for as long as records have been kept. Despite the fact that research on clinical hypnosis with children is still in an early stage of development and the child hypnosis literature is predominantly composed of anecdotal case histories and uncontrolled research studies, one of the best documented uses of hypnosis is in the treatment of pediatric pain where hypnosis has achieved status as an evidencebased/empirically supported intervention. Hypnosis has also been included as one of the psychological interventions recommended by the World Health Organization (WHO) for the management of pediatric procedurerelated cancer pain. In this talk, the current literature and methodogical issues regarding the use of hypnosis in pediatric pain management will be discussed. First the research evidence for the efficacy of hypnosis in the control of acute pediatric pain will be reviewed; following, methodological issues pertinent to the conduct of clinical studies with pediatric populations will be briefly explored; and finally an attempt will be made to summarize and evaluate the existing literature and make suggestions for future studies.

\section{RAPID COMMUNICATION WORKSHOP 3E - ADVANCED QUANTITATIVE METHODS IN PEDIATRIC PAIN RESEARCH \\ Chair: Qian Lu}

\subsection{1 \\ STRUCTURAL EQUATION MODELING IN PEDIATRIC PAIN RESEARCH \\ Q Lu}

University of California, Los Angeles, Los Angeles, California, USA.

This presentation is designed to help participants develop skills in defining and testing plausible structural equation models. Structure equation modeling (SEM) has proven to be one of the most successful methodologies in social and behavioural sciences for specifying and evaluating causal hypotheses with both survey and experimental data. SEM can be used to specify and test hypothesized causal effects relating many sets of variables, while controlling for errors of measurement and other irrelevant sources of variance. Complex research questions often involve the analysis of moderators and mediators. We define moderators and mediators and give an example of a model in pediatric pain research where SEM was utilized to answer a series of questions involving moderators and mediators. In this example, SEM was used to model how parents' anxiety sensitivity influenced laboratory pain responses in children via characteristics of children (ie, mediator), and whether the mechanism differed between boys and girls (ie, sex as a moderator). Through these examples, confirmatory factor analysis (CFA), path analysis, and multiple group comparison techniques are introduced. We will discuss issues facing pain researchers concerning why and when to use SEM techniques, and how to introduce and describe these methods in manuscripts and publications

\subsection{2 INTRODUCTION TO EQS 6.1 FOR WINDOWS E Wu}

Department of Psychology, University of California, Los Angeles, Los Angeles, California, USA.

EQS is known to be one of the most intuitive and comprehensive Structural Equation Modeling (SEM) software available. Although EQS becomes very popular in behavioural medicine, it has not been widely used in pediatric pain research because of researchers' unfamiliarity with EQS software. As part of the workshop of "Advanced Quantitative Methods in Pediatric Pain Research", this session is intended to be a "how to" session using EQS 6.1 for Windows as the demonstration tool.

Some basic concepts such as latent variables will be introduced. The distinctions among various models such as path models, confirmatory factor models, structural equations models, structural mean models, multi-sample models, multi-level models, and mixture models will be illustrated. There are many useful features in EQS such as case selections, transformations, data plotting, and general statistics for data screening. In particular, EQS provides advance missing data diagnosis tools including EM missing data imputation methodology.

EQS has a built-in Diagrammer which allows user to create a SEM model by drawing the conceptual model on the screen. The Diagrammer will create a reusable command file and analytical outcomes are fetched back to the Diagrammer for presentation. We will also discuss and illustrate what to do when data is not normal. This is a very concise hands-on session for Pediatric Pain researchers to use EQS as their research tool. A demo version will be distributed in the workshop so that every attendee will have a chance to experience this wonderful analysis tool. 
4.404.3

LONGITUDINAL ANALYSIS OF PAIN OUTCOMES IN

CHILDREN

\section{T Palermo}

Department of Anesthesiology and Peri-Operative Medicine and Psychiatry, Oregon Health \& Science University

Longitudinal analysis affords many opportunities to answer questions about the changes that occur in children's pain and factors that may predict children's pain outcomes at a later point in time. These questions are relevant both in descriptive longitudinal research as well as in intervention or treatment studies. Dr Palermo will review general considerations in longitudinal data analysis. She will describe multilevel modelling as one method of longitudinal analysis and will present advantages of this approach. An example of multilevel modelling will be presented in which depressive symptoms were tested as a generalized risk factor of the longitudinal course of pain and pain-associated disability in children with chronic disease-related pain. The discussion of longitudinal analysis will conclude with several examples of post hoc probing of moderation and mediation effects. Useful resources for better understanding advanced quantitative methods will be reviewed.

\section{RAPID COMMUNICATION WORKSHOP 4A - PAIN AND SYMPTOM MANAGEMENT IN PEDIATRIC PALLIATIVE CARE Chair: Gustaf Ljungman}

\subsection{1}

\section{PAIN MANAGEMENT IN PEDIATRIC PALLIATIVE CARE WITH SPECIAL EMPHASIS ON CANCER}

\section{G Ljungman}

Pediatric Hematology and Oncology Program, Children's

University Hospital in Uppsala, Sweden

Pediatric Palliative Care comprises the care of children with incurable diseases irrespective of diagnosis, generally with an expectation of reduced survival. The goals of this care are to achieve the best possible quality of life, to reduce discomfort and suffering, to help the child lead an active and normal life as possible at end of life and neither to add, nor to reduce, the number of days of life. Pain management is central in the care of children in palliative care. In a study from Boston it was shown that $89 \%$ of children with cancer, suffered a lot or a great deal from at least one symptom in their last month of life, most commonly pain, fatigue, or dyspnea.

Treatment was successful in only $27 \%$ for pain and $16 \%$ for dyspnea. This part of the workshop will review the current literature and address pain management issues in pediatric palliative care with special emphasis on cancer, and some case scenarios will be discussed.

\subsection{2 \\ INTRACTABLE PAIN AND TERMINAL SEDATION IN CHILDREN \\ Jl Collins}

Pain and Palliative Care Service, Children's Hospital at Westmead, Sydney, Australia

One of the greatest fears of parents for their children receiving palliative care is related to the adequate treatment of pain. One of the major concerns of clinicians caring for children receiving palliative care is related to their ability to manage symptoms adequately, especially in the setting of intractability. Sedation is a recognized therapeutic modality, in the setting of terminal care, when all treatment options for pain relief have been exhausted and refractory pain is managed by reducing conscious awareness. This latter modality of treatment raises questions of ethical concern.

This component of the workshop will discuss the epidemiology of intractable pain in childhood and the range of therapeutic options for children with refractory pain, and the evolving therapeutic options. The pediatric literature will be reviewed. The valid role of terminal sedation will be discussed in this context and an algorithm for its use will be introduced. Case scenarios illustrating issues of ethical concern and the principle of double effect will be discussed.

\subsection{3}

\section{MANAGING PEDIATRIC (NON-PAIN) SYMPTOMS AT THE END OF LIFE}

S Friedrichsdorf, G Ljungman, J Collins

Pain \& Palliative Care Department, Children's Hospitals and

Clinics of Minnesota, Minneapolis, Minnesota, USA

In the United States alone more than 6,500 children die each year due to a life-limiting disease. Data shows the majority of those children suffer from distressing symptoms during their last weeks of life. The provision of current state-of-the-art symptom control is paramount for any health professional working with dying children, but treatment guidelines are sparse. If physical symptoms are not adequately treated it is difficult for children and their families to negotiate the domains of psychological and spiritual care.

This part of the workshop reviews current literature, guidelines and clinical experience regarding the management of integrative and pharmacological therapies of distressing symptoms during the end-of-life period of children and teens, such as anorexia, dyspnea, fatigue, noisy breathing/death rattle, seizures, nausea, and vomiting. Several myths and misconceptions have led to inadequate symptom control in children with a terminal disease. A dying child is often highly symptomatic, and providing professional integrative and pharmacological symptom relief is one of the precincts of pediatric palliative care.

\begin{tabular}{c}
\hline RAPID COMMUNICATION WORKSHOP 4B - \\
NITROUS OXIDE FOR PROCEDURAL PAIN \\
MANAGEMENT IN CHILDREN \\
Chair: Elizabeth Bruce
\end{tabular}

\subsection{1}

\section{SELF-ADMINISTERED AND CONTINUOUS FLOW ENTONOX FOR THE MANAGEMENT OF CHEST DRAIN REMOVAL PAIN}

E Bruce, RF Howard, LS Franck

Pain Control Service, Great Ormond Street Hospital For Children, London, United Kingdom

INTRODUCTION: Morphine is commonly used for chest drain removal pain, although a few studies in adults suggest that inhalation agents may be effective for this procedure (Bruce et al, 2006). Little is known about chest drain removal pain or its management in children. This paper provides a brief overview of three studies which examine the characteristics and management of chest drain removal pain in children. The implications of the findings for future practice and research are also discussed.

METHODS: Study 1 examined the prevalence and clinical characteristics of pain and analgesic practices in 135 nonventilated children aged 1 week to 18 years having chest drains removed. Study 2 was an observation study to determine the efficacy and safety of self-administered Entonox (50\% nitrous oxide and oxygen) for chest drain removal pain in 30 children aged 7-18 years. Study 3 was a pilot randomized controlled trial comparing intravenous morphine and continuous flow Entonox for chest drain removal pain in 14 children aged 3.5 months to 2.75 years.

RESULTS: In study 1, the prevalence of moderate to severe pain during chest drain removal was $76 \%$. Morphine was commonly given preprocedure, but the dose varied considerably. In study 2 , children experienced a significant increase in pain during the procedure compared with preprocedure pain at rest, despite receiving Entonox, morphine and/or diclofenac. However, procedure pain was no worse than preprocedure pain during movement or deep breathing. A few minor side effects occurred, which resolved spontaneously. In study 3, no differences were found in pain between the two treatment groups. Children experienced moderate to severe pain during the procedure, despite receiving Entonox or morphine. CONCLUSIONS: Morphine or Entonox alone are unlikely to provide 
adequate analgesia for chest drain removal pain in children. More research is needed to determine the most effective interventions for this procedure.

\section{REFERENCES}

Bruce E, Franck L, Howard R. The efficacy of morphine and Entonox analgesia during chest drain removal in children. Pediatric Anesthesia 2006;16:203-8.

Bruce E, Howard R, Franck L. Chest drain removal pain and its management: A literature review. Journal of Clinical Nursing 2006;15:145-54.

\subsection{2 \\ SAFE ADMINISTRATION AND DELIVERY OF NITROUS OXIDE FOR PROCEDURAL PAIN MANAGEMENT IN CHILDREN}

\section{S Dowden, $\mathrm{F}$ Babl}

Department Anaesthesia \& Pain Management, Royal Children's Hospital, Melbourne, Australia

Nitrous oxide is a useful addition to the pharmaceutical options for managing procedural pain in children. The short onset and offset, tolerability and safety margins combine to make it an ideal drug. It is essential that staff administering nitrous oxide are suitably trained to select patients appropriately and administer the drug safely. This paper will discuss experience with administration of nitrous oxide for procedural sedation in a tertiary children's hospital. The program for staff training and audit process will be discussed in detail. Our experience to date demonstrates that high quality care can be delivered if a suitable education structure is put in place. Pitfalls and opportunities will be discussed for those setting up a similar program.

A pediatric sedation credentialing program was implemented in the Emergency Departments of a tertiary pediatric hospital and a community hospital. Pre-post audit showed significant changes in staff behaviour following the introduction of the credentialing program. Rates of documentation of informed consent, evidence of performance of preprocedure risk assessment and recording of appropriate vital signs all improved significantly. This model of staff education is being adapted for the staff in general ward areas of the tertiary hospital. A previous attempt to implement a hospital-wide program failed, however the new program is being developed using the Emergency Department experience and further resources have been put in place to optimize its success. Appropriately trained staff are vital to minimize adverse effects and optimize the efficacy and safety of nitrous oxide delivery.

\section{REFERENCES}

Babl FE, Puspitadewi A, Barnett P, Oakley E, Spicer M. Preprocedural fasting state and adverse events in children receiving nitrous oxide for procedural sedation and analgesia. Pediatr Emerg Care 2005;21:736-43.

Babl FE, et al. Scope for improvement: Hospital wide sedation practices at a children's hospital. (In press) Arch Dis Child, 2006.

\section{RAPID COMMUNICATION WORKSHOP 4C - ASSESSING FUNCTIONALITY IN PEDIATRIC CHRONIC PAIN - A CLOSER LOOK Chair: Deirdre Logan}

\subsection{3}

\section{ASSESSING FUNCTION IN PEDIATRIC CHRONIC PAIN SYNDROMES - A CLOSER LOOK}

$\underline{\text { D Logan }}^{1}$, C Eccleston $^{2}$, T Palermo $^{3}$

1Children's Hospital Boston, Harvard University, Boston, Massachusetts, USA; ${ }^{2}$ University of Bath, Bath, United Kingdom; ${ }^{3}$ Department of Anesthesiology and Peri-Operative Medicine and Psychiatry, Oregon Health \& Science University, Portland, Oregon, USA This symposium describes the importance of refining our assessment and understanding of functional disability secondary to pediatric chronic pain

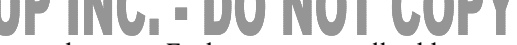

syndromes. Each presenter will address a relevant theme, including (1) defining normative function, (2) describing developmental changes in functional disability, and (3) identifying an approach to micro-level assessment of a single domain of function using the example of school functioning. Professor Eccleston will present a conceptual model of how to understand normal adolescent function, focusing on physical and social function. Data will be presented on the developmental context of normal function, and how adolescents with chronic pain perceive their own development in comparison with peers. Dr Palermo will review literature on age differences in functional disability and will present data on the selfperception of activity limitations across school age and adolescence, highlighting the importance of examining functional disability at different developmental time points. Using an example from a sample of pediatric patients with recurrent headache, Dr Palermo will present data highlighting age-dependent relationships among pain, depressive symptoms and functional disability in children and adolescents. Approaches and study designs necessary for examining age and developmental changes in the functional impact of chronic pain on children and adolescents will be reviewed. Dr Logan will review the literature that conceptualizes school functioning beyond simple absence rates. Data will be presented from an ongoing treatment study, one aim of which is to refine methods of assessing school functioning, incorporating reports from adolescents, parents, teachers, and school administrators. This data will provide preliminary evidence for using such methods to obtain a more detailed understanding of the relation between pain conditions and school functioning. Future directions will be discussed by each presenter to identify approaches through which functional impairment can be assessed to provide a more comprehensive, developmental view of functionality in youth with chronic pain.

\subsection{1}

\section{ASSESSING FUNCTION IN PEDIATRIC CHRONIC PAIN SYNDROMES - A CLOSER LOOK}

D Logan ${ }^{1}$, C Eccleston, T Palermo

1Children's Hospital Boston, Harvard University, Massachusetts,

\section{USA}

This symposium describes the importance of refining our assessment and understanding of functional disability secondary to pediatric chronic pain syndromes. Each presenter will address a relevant theme, including (1) defining normative function, (2) describing developmental changes in functional disability, and (3) identifying an approach to microlevel assessment of a single domain of function using the example of school functioning. Prof Eccleston will present a conceptual model of how to understand normal adolescent function, focusing on physical and social function. Data will be presented on the developmental context of normal function, and how adolescents with chronic pain perceive their own development in comparison with peers. Dr Palermo will review literature on age differences in functional disability and will present data on the self-perception of activity limitations across school age and adolescence, highlighting the importance of examining functional disability at different developmental time points. Using an example from a sample of pediatric patients with recurrent headache, Dr Palermo will present data highlighting agedependent relationships among pain, depressive symptoms and functional disability in children and adolescents. Approaches and study designs necessary for examining age and developmental changes in the functional impact of chronic pain on children and adolescents will be reviewed. Dr Logan will review the literature that conceptualizes school functioning beyond simple absence rates. Data will be presented from an ongoing treatment study, one aim of which is to refine methods of assessing school functioning, incorporating reports from adolescents, parents, teachers, and school administrators. This data will provide preliminary evidence for using such methods to obtain a more detailed understanding of the relation between pain conditions and school functioning. Future directions will be discussed by each presenter to identify approaches through which functional impairment can be assessed to provide a more comprehensive, developmental view of functionality in youth with chronic pain. 
4.403.2

\section{ASSESSING FUNCTION IN PEDIATRIC CHRONIC PAIN \\ SYNDROMES - A CLOSER LOOK}

\section{ADOLESCENT DEVELOPMENT AND CHRONIC PAIN}

\section{Eccleston}

University of Bath, Bath, United Kingdom

Many chronic pain intervention studies aim to return adolescents to 'normal' everyday function, despite pain. However, it is rarely established what normal adolescent activities are. In addition, where normal activities are assumed it is often unclear how one should understand adaptation or change to engagement in normal activity. Prof Eccleston will present a conceptual model of how to understand normal adolescent function, focusing on physical and social function. Data will be presented on the developmental context of normal function, and how adolescents with chronic pain perceive their own development in comparison with their peers. Predictive data will be analyzed on what determines self-perception of developmental stage in teenagers. Finally, a conceptual model will be developed on how to understand the effects of chronic pain on normal everyday function.

\subsection{3 \\ AGE AND DEVELOPMENTAL CHANGES IN FUNCTIONAL DISABILITY DUE TO CHRONIC PAIN}

\section{T Palermo}

Department of Anesthesiology and Peri-Operative Medicine and Psychiatry, Oregon Health \& Science University, Oregon, USA

Age has been identified as a potentially important factor affecting the prevalence, severity, and functional impact of chronic pain on children and adolescents. There is preliminary evidence that increasing child age is associated with more decrements in functioning and reduced quality of life in children and adolescents with chronic pain. Dr Palermo will review the literature on age differences in functional disability and will present data on the self-perception of activity limitations in school age children and adolescents. Dr Palermo will also discuss the importance of examining the role of functional disability at different developmental time points. Using an example from a sample of pediatric patients with recurrent headache, Dr Palermo will present data highlighting age-dependent relationships among pain, depression and functional disability in children and adolescents. In particular, findings that functional disability served as a mediator of the relationship between headache pain and depression for children more strongly than for adolescents will be discussed. Dr Palermo will discuss factors that develop with age including self-concept and coping abilities that may play a role in how children and adolescents are impacted by pain. Finally, Dr Palermo will review approaches and study designs necessary for examining age and developmental changes in the impact of chronic pain on children and adolescents.

\section{RAPID COMMUNICATION WORKSHOP 4D - PSYCHOSOCIAL CORRELATES OF HEADACHE IN CHILDREN$$
\text { Chair: Manju Mehta }
$$

\subsection{5}

\section{PSYCHOLOGICAL CORRELATES OF HEADACHE IN CHILDREN}

\section{Mehta, R Sagar, M Mongia}

Department of Psychiatry, All India Institute of Medical Sciences,

\section{New Delhi, India}

Pain is a multidimensional phenomenon that encompasses biological, psychological and social domains. It is not surprising to witness incidences of pain in pediatric population. In fact there has been a recent upsurge in reports of headache in children in the developing world. The prevalence rates of headache in children range from $1 \%$ to $25 \%$ world wide. Headache has been underestimated and undertreated in this section of the society.
Headache itself is a painful and often disabling feature. The most common types of headache-tension type headache (TTH) and migraine cause substantial level of disability in the sufferers. Prior research in this area has proven the role of psychosocial factors in the causation of headache in pediatric population. The cultural nuances in causation and maintenance of headache and peculiarities of headache in our population will be discussed.

\subsection{1}

\section{PSYCHOLOGICAL STRATEGIES IN THE MANAGEMENT OF HEADACHE}

\section{Mehta, R Sagar, M Mongia}

Department of Psychiatry, All India Institute of Medical Sciences, New Delhi, India

As psychological correlates contribute to the manifestation of headache, the treatment needs to be addressed to modify the psychological factors. There are many reports and WHO bulletins advocating psychological management of headache. In developing countries, the patients' cultural conditioning often leads to questioning of the application and efficacy of psychological treatments. We have studied the efficacy of cognitive behaviour therapy and play therapy in the management of headache. Comprehensive assessment of etiological and maintaining factors is necessary to understand. In our population, problems related to academic tasks, high achievement and poor social skills are often seen in headache patients. 77 children with tension-type headache and migraine in the age range of $8-15$ years, both genders were treated by cognitive behavioural methods. Pre-and post-treatment assessments were made on both objective and subjective measures. Patients were treated individually and were trained to use strategies such as relaxation therapy, cognitive restructuring, problem solving and study skills. Family members were involved as cotherapists. All the patients had reduction in frequency, intensity and duration of headache. The need for medication was decreased. Certain family and individual psychosocial factors facilitated the outcome.

\subsection{2}

\section{PSYCHIATRIC ASPECTS OF HEADACHE}

M Mehta, R Sagar, M Mongia

Department of Psychiatry, All India Institute of Medical Sciences, New Delhi, India

The relationship between headache and psychopathology has frequently been discussed clinically. Headache as a psychosomatic symptom may be present in depression in childhood and adolescence. Cognitive processes play an important role in the causation of headache. Any episode of depression that occurs in the period before the onset of pain is liable to have psychological residues, which predisposes an individual to become helpless and depressed. Depressive cognitions precede the ideas of helplessness. These ideas are, in turn, facilitated by pain. This intricate cycle will be discussed in detail. In our study, out of the 50 cases studied $56 \%$ had one or more type of psychopathology. Depression was the most common psychopathology. It was twice more common in males than females. Hyperkinesis was characteristically present in males only. Primary diagnoses in these children were made according to ICD 10. These included persistent somatoform pain disorder (72\%), depression( $16 \%)$, emotional disorder, dissociative disorder and conduct disorder in $6 \%, 4 \%$ and $2 \%$ respectively. Depression was 7 times more in males than females. Statistically significant association was seen between depression and headache.

\subsection{3}

\section{ROLE OF STRESSFUL LIFE EVENTS IN HEADACHE}

M Mehta, R Sagar, $\underline{M}$ Mongia

Department of Psychiatry, All India Institute of Medical Sciences, New Delhi, India

Headache problems in children often result due to stress factors. Stress in daily life may also maintain this problem. Various stressful life events ranging from academic stress to stress in interpersonal relationships to presence of chronic illness in the family may lead to decreased use of coping 
resources, hence, leading to stress and headache. Poor frustration tolerance and inability to cope with stress may also manifest in the form of psychiatric disorders such as anxiety and depression. Headache may then be a component of these disorders. Familial ways of dealing with stress and the role of family factors in etiology of headache in children have been seen to play a role in causation and maintenance of headache. Three studies to assess the role of stress in headache were conducted in our department. In the first study, 2000 children in the age range of 8-14 years, both from government and public schools, were assessed for stress and coping strategies using standardized psychological scales. In this study, stress was clinically manifested through somatic and psychological complaints. $27 \%$ of public school children and $25.5 \%$ of government school children had mild stress. $10.2 \%$ of public and $6.7 \%$ of government school children had moderate to severe stress. Around $49 \%$ children had headache, others complained of stomach ache, abdominal pain, nausea, vomiting. etc. Daily stresses were experienced by both the groups. Indian patients prefer somatic language to express inner psychological distress. In the second study, nearly, $50 \%$ of the adolescents studied reported somatic symptoms such as headache, body ache, and weakness as their chief complaints. In the third study, out of the 50 children and adolescents studied, $48 \%$ had headaches alone and if combined with other somatic complaints it was present in $80 \%$ of the cases. Males outnumbered females.

\section{RAPID COMMUNICATION WORKSHOP 4E - STANDARDIZING THE ASSESSMENT OF LONG-TERM ALTERATIONS IN PAIN PROCESSING AND DEVELOPMENT}

\author{
Chair: Dick Tibboel
}

\subsection{1 \\ LONG-TERM EVALUATION STUDIES OF NEONATAL PAIN EXPOSURE}

RHIA Schouw, JBW Peters, D Tibboel

Department of Pediatric Surgery, Erasmus MC - Sophia Children's Hospital, Rotterdam, The Netherlands

Evaluation of the consequences of infant pain is an important concern, especially in premature neonates who are exposed to prolonged and repeated pain during their hospitalization. One frequent used method to evaluate long-term effects is a retrospective study. Historical cohorts are used and evaluated. The methodological pitfalls compared to prospective follow-up studies will therefore be addressed. Moreover, which outcome data is of most importance?

The few studies that have been evaluating long-term pain threshold alterations use a variety of methods. Which pain stimuli are most suited to induce and evaluate experimental pain? The same question will be answered for clinical pain. Moreover, not every method is suited for each age group. Problems of evaluating pain thresholds in specific groups of children will therefore be discussed. To answer specific burning questions concerning pain sensitivity alterations, some methods are better than others. Until now there is no consensus in the used algorithms. Should we study pain thresholds and suprapain thresholds, or must we focus on habituation and sensitization instead. We will address the methodological pitfalls of the various pain assessment algorithms and how they can be resolved.

\subsection{2 \\ BEHAVIOURAL ALTERATIONS FOLLOWING NEONATAL PAIN EXPOSURE}

RHJA Schouw, IBW Peters, D Tibboel

Department of Pediatric Surgery, Erasmus MC - Sophia Children's Hospital, Rotterdam, The Netherlands

Since the pain experience is multifactorial, not only the development of the pain system will be altered, but the long-term social emotional functioning might as well be affected. Questions like "which methods are suited to assess long-term social emotional function in children and which psychological factors are of most importance?" "Could neonatal pain expo-
DAP. DONOT COPY

sure alter children's behaviour?" "How should these factors be evaluated?" "What are the pitfalls of possible psychological confounders in long-term evaluation studies?" will all be addressed. Moreover parental factors, ie, stress, anxiety, depression, etc, might influence children's pain sensitivity and their coping with pain. What is the importance of parental psychosocial factors and how should they be assessed and interpreted? Lastly, in a round table discussion we hopefully come closer to consensus on future standardization of long-term evaluation of neonatal pain experiences.

\section{PLENARY 5 - ISSUES IN PEDIATRIC PAIN}

\subsection{1 .1}

\section{FAMILIES AND PEDIATRIC PAIN: BACK TO THE FUTURE}

\section{CT Chambers}

Departments of Pediatrics and Psychology, Dalhousie University and IWK Health Centre, Halifax, Nova Scotia

The family plays a critical role in caring for children in pain. Research on the role of the family in children's acute and chronic pain has grown exponentially in recent years. Despite advances in our understanding of the role of the family in pediatric pain, research in the area has been stalled by lack of an underlying theoretical framework for understanding family factors in relation to pain. This presentation will summarize recent research findings regarding the role of the family in pediatric pain (with an emphasis on studies published since the last International Symposium on Pediatric Pain in 2003) and will describe a comprehensive framework developed by Palermo and Chambers (2005) for conceptualizing the role of the family in pediatric pain. To provide direction on how research on families and pain could be better integrated in the future, ways in which this framework can be used to guide future research on families and pediatric pain will be discussed.

\subsection{1 .2}

\section{PAIN ISSUES IN CHILDREN OF THE DEVELOPING WORLD} AT Bösenberg

Department of Anaesthesia, University of Cape Town, South Africa

Evidence-based medicine, defined as the "the conscientious, explicit and judicious use of current best evidence in making decisions about the care of individual patients", has little relevance for children uncertain of their next meal let alone dose of analgesia. The purpose of this lecture will be to outline issues related to pain management of children in the developing world based on my own experience and those of colleagues who have worked in a diversity of developing countries.

Since the widespread acceptance that children do actually feel pain, there has been dramatic improvement in acute and chronic pain management in children, particularly those in the developed world. The realization that pain prevention and treatment can reduce both morbidity and mortality has led to a search for better analgesic methods. Dedicated acute pain services in many pediatric institutions have helped develop a systematic approach to patient assessment, treatment and quality assurance. Effective pain management is now the expectation in the developed world.

Unfortunately pain management of children in a first world environment is vastly different to those available to practitioners working with limited resources and inadequately trained personnel in the developing world. Attempting to apply similar standards is fraught with difficulty. Literacy, malnutrition, poor cognitive development, differing copying strategies, pharmacogenetic, cultural and language differences all add to the complexity of the problem.

Children of the developing world are faced with vastly different problems. These range from limited access to health care, different disease spectrum (HIV, malaria, sickle cell disease) and delays in diagnosis. Children are victims of poverty, malnutrition, violence (war, trauma, abuse), and their attitude towards pain, and tolerance thereof, is different.

Simple pain management strategies may produce the most benefit with the least risk. More complex techniques, that offer the most benefit, require a minimum standard of monitoring and regular reassessment to allow indi- 
vidualized titration of analgesia. But these are seldom available to children of the developing world. The final choice of analgesia, unfortunately, is dictated by economic pressures or by the facilities available rather than what would be considered best for the child. Nonetheless it is morally, ethically and physiologically beneficial to provide children with effective analgesia despite the immense inequalities that exist in our world.

\subsection{3}

\section{ISSUES IN NEONATAL PAIN}

\section{B Stevens}

Faculties of Nursing and Medicine, University of Toronto; The Hospital for Sick Children, Toronto, Ontario

Important strides have been made in our understanding of pain mechanisms, the immediate and short-term consequences of pain, the proliferation of pain assessment measures and the safe and effective pain management strategies, particularly for acute neonatal pain. We have witnessed important discoveries related to the spinal, central and behavioural mechanisms of pain during early human development, a clearer understanding of the consequences of pain to infants born very prematurely and adaptations that transpire over time, pain indicators for acute procedural and post-operative pain and the inclusion of physiologic biomarkers of pain and stress, and determining the safety and efficacy of a variety of pharmacological, physical, environmental and behavioral interventions both alone and in combination. Furthermore, international recognition and efforts by professional, national and local organizations to develop pain management guidelines, based on systematic reviews or meta-analyses, have had a considerable impact on pain assessment and management in neonates. However, despite these advances, pain remains under-recognized and under-managed for many neonates and infants in a variety of clinical and community settings.

Attention to: (a) the developmental capacity for experiencing pain (including the fetus); (b) assessing persistent pain due to inflammatory, visceral, or central sources, (c) validation of unique indicators for pain assessment in vulnerable infant populations (extremely low birth weight, critically ill, neurologically impaired and end-of-life); (d) novel approaches to pain management (pharmacologic, behavioural, physical, complementary and alternative) and factors that determine analgesic effectiveness; (e) environmental and contextual factors that affect pain perception and response in neonates; (f) parental involvement in neonatal pain management, (f) infant pain assessment and management in developing countries; and $(\mathrm{h})$ issues that deal with health policy and economics related to neonatal pain will provide a starting point for those professionals interested in improving their knowledge, communication, critical analyses and decision making with regard to pain in neonates. Our ultimate goal is to prevent, minimize or ameliorate pain and its consequences for all infants.

\section{WORKSHOP 4A - PSYCHOSOCIAL COMPONENTS OF ADOLESCENT PAIN AND DISABILITY \\ Chair: Jan Passchier}

\subsection{1 \\ PSYCHOSOCIAL COMPONENTS OF ADOLESCENT PAIN AND DISABILITY \\ J Hatchette'; J Guite'; B Larsson ${ }^{3}$; L Passchier ${ }^{4}$; D Logan ${ }^{5}$ ${ }^{1}$ IWK Health Centre, Halifax, Nova Scotia; ${ }^{2}$ The Children's Hospital of Philadelphia, Philadelphia, Pennsylvania, USA; ${ }^{3}$ Norwegian University of Science and Technology, Trondheim, Norway; ${ }^{4}$ Erasmus Medical Centre, Rotterdam, The Netherlands; ${ }^{5}$ Children's Hospital Boston and Harvard University Medical School, Boston, Massachusetts, USA}

Recurrent pain is one of the most commonly reported health complaints in adolescence. Although the prevalence of stomach pain decreases during puberty, the incidence of back, limb and head pain increases throughout adolescence. Chronic pain is also frequently reported among this age group.

The social context of children and adolescents' pain experiences has a powerful impact on how pain responses and disability are learned. The socialization of children's pain begins quite early and both professional opinion and psychological theory indicate that the family is instrumental in influencing children's pain experiences. For example, parent and caregiver responses to children's everyday pain (ie pain from minor cuts and falls), as well as repeated exposure to family members' pain episodes, provide children with valuable information about social display rules, appropriate pain responses and coping strategies. By the time children reach adolescence they have acquired numerous learning experiences that have shaped how they will cope with pain. Chronic pain is often debilitating for children and adolescents, putting them at greater risk for psychosocial, psychiatric and functional problems. For example, both parental divorce and low peer contact have been associated with frequency of pain complaints; both children and adolescents with recurrent abdominal pain or headaches suffer higher levels of anxiety and depressive symptoms; adolescents with migraine or tension-type headaches report greater school absence. How young adolescents choose to resolve or cope with chronic pain is a function of many factors, but perhaps most influential is the family. Two psychological mechanisms, widely explored and supported in the pain literature, account for much of the impact of the family on children's pain behavior. These are social modeling and parental reinforcement. Multiple exposures to pain incidents provide children with opportunities for the modeling and reinforcement of familial and cultural beliefs and attitudes about how to experience and cope with trauma, disease and pain.

Modeling and reinforcement are clearly evidenced in the pain literature. Children and adolescents with unexplained chronic pain report more pain models than those with explained pain and the aggregation of pain complaints within the family underscores the impact of physical proximity and shared environment on pain expression and disability. Parental modeling and reinforcement of illness behaviors clearly influence both the development and maintenance of frequent somatic complaints in children who are otherwise healthy. Mothers in particular seem to be an influential source of children's pain behaviors.

Peers may also play an important role in how adolescents learn about pain responses and disability. Perceptions of the cause and severity of pain have been shown to be influential in how children respond to peers' pain. For example, from a very young age, children understand the difference between symptoms in the presence or absence of organic disease, perceive symptoms with organic cause as more severe, are less likely to relieve peers of responsibility when physical complaints are perceived to be without organic cause and demonstrate gender-role expectations for peers' pain expression and disability. However, how the perceptions of others' pain and disability might mediate one's own pain responses and disability is unclear.

Despite the evidence for social modeling and reinforcement in pain responses and disability, how these psychosocial mechanisms relate to adolescents' pain experiences, comorbidity of pain and the psychiatric dimensions related to pain remain poorly understood. By adolescence, young people begin the developmental, cognitive, psychological and social shift toward autonomy. Less time is spent with parents and peers increasingly become the primary source of information about a range of attitudes and behaviors, many relating to health. Peers also become an essential source for approval when health-related decisions are being made. During this transition from childhood to adulthood, adolescents begin to make independent decisions about participation in school, leisure activities and work and thus begin to directly mediate their own disability. What the literature lacks to date is an understanding of how parent-adolescent and peeradolescent relationships impact the adolescents' experiences of pain and accompanying disability and psychiatric morbidity.

To this end, the proposed workshop aims to explore the unique psychosocial and psychiatric aspects of the adolescents' pain experience. The specific focus of this workshop will be to examine the social influence that parents and peers have in adolescents' pain expression, disability and psychiatric symptoms. Existing literature will be briefly reviewed and completed research (both qualitative and quantitative) as well as research presently being undertaken will be presented to provide a broader understanding of 
the psychosocial factors impacting pain and disability among adolescents. Topics to be covered will include:

1. An examination of the role of parents in encouraging illness behavior and functional disability in adolescents with chronic pain via discussion of parent and adolescent psychological symptoms as predictors.

2. An examination of the parent-adolescent relationship as a predictor of parental encouragement of illness behavior and functional disability via parental perceptions of adolescent distress.

3. An examination of overall parenting stress, spousal relationship stress and general life stress as predictors of parental encouragement of illness behavior and adolescent functional disability.

4. An examination of how direct observation of a peer's pain experience and multiple peer pain models function as potential sources of social modeling for adolescents' pain responses.

5. An examination of the extent to which peer likeability mediates pain expression among adolescents.

6. An examination of the extent to which gender role expectations impact adolescents' pain responses and disability.

7. An examination of peer and parental factors on psychiatric symptoms related to recurrent and chronic pain.

8. An examination of the relationship of pain comorbidity with psychiatric disorders, such as anxiety and depression, among adolescents.

9. An examination of the directionality of recurrent pain complaints and associated psychiatric disorders.

\subsection{1}

\section{THE ADOLESCENT WITH PAIN AND THE SOCIAL ENVIRONMENT: HOW BAD IS GOOD?}

\section{J Passchier, JAM Hunfeld}

Department of Medical Psychology and Psychotherapy, NIHES Erasmus University Medical Center Rotterdam, the Netherlands BACKGROUND: According to Fordyce, paying attention and being nice in response to manifestations of pain by a chronic pain patient is a bad strategy because it reinforces the pain behaviour and leads to more frequent pain manifestations. This is shown to be maladaptive and, therefore, in case of chronicity pain should be ignored and healthy behaviour rewarded instead. However, the learning model, though successfully applied in severe chronic patients in pain clinics, is not necessarily indiscriminately useful for chronic pain in children and adolescents from a general population.

PURPOSE: To demonstrate the influence of parents on adolescents with chronic pain and vice-versa.

METHODS: We will give several examples from our cohort and intervention research that indicate negative consequences of a diminished attention of parents to the (chronic) pain of their child.

RESULTS: More attention of parents and peer to this child in a pain-free state was even associated with more pain. Also the quality of life of the child with chronic pain was positively related to the extent to which pain behaviour was 'rewarded' by the parents or the peers. It looks as if parents and peer usually ignore the chronic pain of the adolescent instead of being overconcerned about it. This interpretation is in line with our interviews with these adolescents: they state that they avoided to speak about their pain problems because of a perceived lack of understanding by others. It is also in conformity with our finding that from the dimensions of the Impact on Family Scale, the mastery of the mother with her child's pain showed most problems. Therefore, we performed an evaluation of a cognitive behaviour program for adolescents with chronic pain with two modules that were devoted to educate parents how to cope with that pain. A preliminary finding was that the functioning at home of the juvenile patients was improved after the training in comparison with a waiting-list control group. When interviewed the parents expressed the usefulness of the sessions and that they felt helped to support their child better.

RECOMMENDATION: We recommend to focus more on the benificial role that parents and peers can have in the management of juvenile chronic pain.

\subsection{2}

PSYCHOLOGICAL SYMPTOMS, ADOLESCENT-PARENT

RELATIONSHIPS, AND PARENTING STRESS IN THE CONTEXT OF ADOLESCENT CHRONIC PAIN

\section{JW Guite, D Logan}

Children's Hospital Boston, Harvard University, Boston,

Massachusetts, USA

Adolescents with chronic pain conditions are at greater risk for psychosocial and functional problems. Parents of adolescents with chronic pain have been found to experience elevated levels of pain, somatic symptoms and emotional distress. Parental modelling and the extent to which parents reinforce or encourage pain and illness behaviours for the adolescent have been associated with the adolescent's experience of pain and disability. However, the complex relationships among adolescent and parent psychological symptoms, parent-adolescent relationships, and parenting stress as they relate to an adolescents' experience of pain and accompanying disability remain poorly understood.

This portion of the workshop will briefly review relevant literature pertaining to this topic and will present original research that explores adolescent and parent psychological symptoms, the parent-adolescent relationship, and parenting stress as predictors of parental encouragement of pain behaviour and adolescent functional disability among adolescents with nondisease-specific chronic pain conditions. The discussion will more specifically focus around:

1. An examination of adolescent and parent self-reported psychological symptoms as predictors of parental encouragement of pain behaviour and functional disability in adolescents with chronic pain.

2. An examination of parental perceptions of adolescent distress and the parent-adolescent relationship as predictors of parental encouragement of pain behaviour and adolescent functional disability.

An examination of the parents' experience of stress associated with parenting an adolescent with chronic pain, spousal relationship stress and general life stress as predictors of parental encouragement of pain behaviour and adolescent functional disability.

\begin{tabular}{c}
\hline WORKSHOP 4B - OPIOID AND \\
BENZODIAZEPINE WITHDRAWAL: \\
A CHALLENGING SIDE EFFECT OF PAIN \\
CONTROL IN THE CRITICAL CARE SETTING \\
Chair: Linda Franck \\
\hline
\end{tabular}

5.202

OPIOID AND BENZODIAZEPINE WITHDRAWAL: ADVERSE EFFECTS OF PAIN CONTROL IN CRITICAL CARE

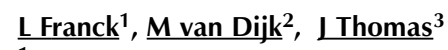

${ }^{1}$ Institute of Child Health and Great Ormond Street Hospital, London, United Kingdom; ${ }^{2}$ Department of Pediatric Surgery, Sophia Children's Hospital, Rotterdam, The Netherlands; ${ }^{3}$ Red Cross War Memorial Children's Hospital, Cape Town, South Africa Opioids and benzodiazepines are commonly used in the paediatric intensive care setting to relieve pain and anxiety and to blunt physiological stress responses to invasive therapies. Unfortunately, the use of these drugs for more than a few days may lead to tolerance and physical dependence. Withdrawal symptoms, including central nervous system hyperirritability, autonomic nervous system dysregulation, gastrointestinal dysfunction respiratory distress and abnormal motor movements, have been documented in critically ill infants and children for over 20 years. Iatrogenic withdrawal symptoms, caused by too rapid tapering off or abrupt discontinuation of opioids or benzodiazepines complicates medical treatment and may prolong recovery and hospital stay. Despite awareness of the problems, there little evidence to guide treatment decisions or consensus on methods to evaluate new therapies.

This workshop consists of three presentations, which will provide an overview of the state of research and clinical practice regarding the assessment of withdrawal in different neonatal and paediatric intensive 
care settings, present new findings relevant to clinical practice, and stimulate discussion of the challenges, barriers and opportunities for optimal prevention and management.

DISCUSSION: The workshop speakers and participants will engage in open discussion on the issues brought forth in the presentations. The following questions are suggested as a further stimulus for debate:

1. What are the most important characteristics of a withdrawal assessment in children receiving long term analgesia and sedation?

2. Is the evidence sufficient to propose that all tapering and discontinuation of long term opioids and/or benzodiazepines be based on standardised assessment?

3. What are the biggest challenges in changing intensive care unit practices in relation to analgesia and sedation?

\subsection{1}

\section{VALIDITY, RELIABILITY AND MAJOR CONFOUNDERS IN} WITHDRAWAL ASSESSMENT

\section{Franck}

UCL Institute Of Child Health, Great Ormond Street Hospital, London, United Kingdom

This presentation will briefly review the historical development of withdrawal symptom assessment in infants exposed to opioids in utero and discuss the current evidence base for opioid and benzodiazepine withdrawal in infants and children receiving intensive care and long-term analgesia and sedation. Assessment tools and procedures will be compared and validity and reliability concerns will be highlighted. Major confounding factors and sources of bias in the development and evaluation of withdrawal assessment will be examined, including the lack of specificity for some of the symptoms, variation in treatment, and biases related to serial assessment procedures. Attitudinal and organizational factors will also be considered. Methods for addressing these obstacles will be suggested and a new model for research on withdrawal assessment will be proposed. Recent data from a randomized trial of an intervention to improve pediatric intensive care unit sedation management for children with acute respiratory failure will be used to illustrate more robust methods for establishing validity and reliability of withdrawal assessment tools.

\subsection{2 .2 \\ WITHDRAWAL SYMPTOMS AFTER ECMO TREATMENT: EXPERIENCES IN DAILY PRACTICE \\ M van Dijk, D Tibboel}

Department of Pediatric Surgery, Erasmus MC - Sophia Children's Hospital, Rotterdam, The Netherlands

BACKGROUND: Our PICUs admit a great many patients whose conditions require prolonged administration of opioids and benzodiazepines. Among these patients are those treated with extracorporeal membrane oxygenation (ECMO). Withdrawal is a major concern in this patient group.

PURPOSE: We aimed at assessing incidences of withdrawal symptoms in relation to cumulative opioids and benzodiazepines treatment. In addition we sought to collect parental information on treatment of withdrawal in clinical practice.

METHODS: We present a tripartite approach. First, we report on a retrospective evaluation of amounts of methadone and clonidine administered to patients after ECMO. Second, we present findings from a prospective study in ECMO and other PICU patients over six months. For 79 patients who received continuous morphine and/or midazolam for more than five days, we completed checklists on withdrawal symptoms twice a day. We focused on symptoms related to the central and autonomous nervous systems, and gastrointestinal tract. Third, we will describe two case studies of neonates with CHD requiring ECMO. Both showed serious withdrawal symptoms after indispensable use of clonidine and methadone, and were placed on a weaning protocol. In consultation with the parents a weaning schedule of methadone was introduced after discharge. We interviewed the parents on their experiences to get an insight into the parents' perspective as well, and report on the problems in daily practice.
5.202.3

ASSESSMENT ISSUES IN PATIENTS WITH LONG TERM PAIN IN RESOURCE LIMITED SETTINGS

\section{IThomas}

This presentation will describe challenges in withdrawal assessment in children with serious burn injuries who require analgesia and sedatives for a considerable time. However, in a developing country with scarce resources, these drugs may often be discontinued too abruptly. In addition to unreliable access to medication, there are also problems with adequate training of clinical staff and the provision of expert consultation. Cases will be used to illustrate the local and governmental issues that affect assessment and treatment of withdrawal. Prospective and retrospective data will be presented from standardised scoring of withdrawal symptoms in children with greater than $40 \%$ total body surface area burns to highlight the challenges.

\begin{tabular}{c}
\hline WORKSHOP $4 \mathrm{C}$ - PARENTS OF CHILDREN \\
IN PAIN: THE INTERRELATIONSHIP OF \\
PARENTAL STRESS, PARENTAL COPING AND \\
CHILD PAIN \\
Chair: Christopher Eccleston \\
\hline
\end{tabular}

\subsection{1}

THE IMPORTANCE OF PARENTAL EMPATHY IN PEDIATRIC PAIN

$\underline{\text { L Goubert }}$

University of Ghent, Ghent, Belgium

In this presentation the role of parental empathy and parental distress in pediatric pain will be explored. It is proposed that distress and empathy have an evolutionary basis and value, in that they instigate tendencies to help (irrespective of its instrumental value) and increase feelings of interpersonal connection. The concept of parental empathy will be discussed in relation to a number of theoretical models, including the Perception Action Model (PAM; Preston \& de Waal, 2002). A new theoretical model of empathy applied to pain will be proposed that takes into account the cognitive, affective and behavioural dimensions of empathy (Goubert et al, 2005). Further, data will be presented investigating the role of parental empathic responses in both populations of healthy children and children with chronic pain. The results of these studies will be discussed in relation to existing theories and how such understanding can contribute to our understanding of the roles parents play in the treatment of acute and chronic pediatric pain.

\subsection{2}

PEDIATRICS, PAINFUL PROCEDURES, AND PARENTS: REASSURING INTERACTIONS BETWEEN CHILDREN IN PAIN AND THEIR CAREGIVERS?

\section{McMurtry}

Dalhousie University, Halifax, Nova Scotia

This talk will review major research findings on the different ways in which parents respond to their child when he/she is undergoing a painful procedure. Specific attention will be devoted to exploring parental reassurance and its counterintuitive relationship with child distress in acute pain situations (eg, immunization). Potential mechanisms of the relationship between reassurance and child distress will be explored. The speaker will present a detailed description of parental reassurance including a categorization of potential subtypes [eg, signal of procedure completion ("it's all done") versus permitting emotional expression ("it's okay to cry")]. In addition, two linguistic qualities (speech function and tone) of spontaneous parental reassurance will be explored. The implications of the research findings will be discussed with respect to improving both child and parent outcomes. Ms McMurtry will make recommendations regarding the adaptive value of specific parental behaviours in the presence of children undergoing a painful procedure. Future research directions will also be discussed. 


\subsection{3 THE PARENTAL EXPERIENCE OF PEDIATRIC CHRONIC PAIN}

\section{A Jordan}

University of Bath, Royal National Hospital for Rheumatic Diseases, United Kingdom

Existing literature has identified the widespread deleterious impact of pediatric chronic pain on the everyday lives of children and adolescents. Studies have shown that young people with chronic pain report impairments in numerous areas of their lives including physical, emotional, social and familial functioning. Recent research has focused on the experience of pediatric pain within a wider family context (Chambers, 2003), with studies examining the experience of parents as primary caregivers.

Parenting a child or adolescent with chronic pain has both an extensive and significant impact on parental life. Research findings have demonstrated that parents of adolescents with chronic pain report elevated levels of emotional distress and impairments in social functioning (Hunfeld et al, 2001; Eccleston, Crombez, Scotford, Clinch \& Connell, 2004). Additional studies have identified that parenting a child or adolescent with chronic pain imposes restrictions on parental career progression and family finances (Sleed, Eccleston, Beecham, Knapp \& Jordan, 2005; Akobeng, Suresh-Babu \& Thomas, 1999). Furthermore, parents report alterations in their parenting style as a result of caring for a young person with chronic pain (Jordan, Eccleston \& Osborn, in press).

The aims of this session are threefold. The first aim is to provide a comprehensive overview of existing literature regarding the extensive impact of adolescent chronic pain on parental life and the wider impact on the family. Secondly, this session proposes to explore the nature of the association between parental and child outcomes in pediatric chronic pain. Finally, this session intends to provide a discussion of the implications of existing research findings with respect to improving outcomes for children and adolescents with chronic pain and their parents.

\section{WORKSHOP 4D - WHY SOCIAL PAIN HURTS: THE ROLE OF NEURAL, LINGUISTIC AND PSYCHOLOGICAL FACTORS Chair: Allen Finley}

\subsection{4}

WHY SOCIAL PAIN HURTS: THE ROLE OF NEURAL, LINGUISTIC AND PSYCHOLOGICAL FACTORS

\section{Piira $^{1}$, M-C Grégoire ${ }^{2}$, I Clemente ${ }^{3}$}

${ }^{1}$ Sydney Children's Hospital and University of New South Wales, Sydney, Australia; ${ }^{2}$ IWK Health Centre, Dalhousie University, Halifax, Nova Scotia; ${ }^{3}$ UCLA Center for the Study and Treatment of Pain, Pediatric Pain Research Program, Department of Pediatrics, D Geffen School of Medicine at UCLA, Los Angeles, California, USA Social pain may be defined as the pain experienced upon social injury when relationships are threatened, damaged or lost. Not only are similar expressions used to describe the experience of social pain and physical pain (eg, "my heart aches", "it pains me to..."), but there is increasing evidence that the similarities go further, with the possibility of shared central neural circuitry (Eisenberger et al, 2003, 2004).

This workshop will provide an overview of some of the recent work from the neuroimaging literature, as well as insights from the fields of linguistics, anthropology, and psychology.

The chairman and presenters in this session are from diverse professional backgrounds, including anesthesiology, pediatrics and psychology. Together with a multidisciplinary audience, this workshop will explore the complex inter-relationships between social pain and physical pain.

The aims of this workshop are as follows:

1) To review and discuss the similarities between social and physical pain at the physiological level, both in animal and humans.

2) To explore, using interaction with the participants and the results of a

recent survey, the linguistic overlap between expressions used to describe physical and social pain.

3) To present the recent theories explaining why social pain hurts and the animal and human responses to social pain and exclusion, focusing on children when data are available.

4) To identify specific situations of social pain in children, like bullying and neglect, and areas where more research is needed.

The chairman will facilitate an interactive dialogue between the presenters and the participants throughout the workshop, including a question period.

\section{REFERENCES}

Eisenberger NI, et al. Science 2003;302:290-2.

Eisenberger NI, Lieberman MD. TRENDS in Cognitive Sciences, 2004;8:294-300.

Enfield NJ, Wierzbicka A. Pragmatics and Cognition, 2002;10:1-25.

MacDonald G, Leary MR. Psychological Bulletin, 2005;131:202-23.

MacDonald G, Kingsbury R, Shaw S. Adding insult to injury: Social pain theory and response to social exclusion. In: Williams KD, Forgas JP, von Hippel W, eds, The social outcast: Ostracism, social exclusion, rejection and bullying. New York: Psychology Press, 2005.

\subsection{1 \\ SOCIAL PAIN AND PHYSICAL PAIN: A LINGUISTIC IDIOSYNCRASY OR SHARED NEURAL CIRCUITRY?}

T Piira ${ }^{1}$, M-C Grégoire ${ }^{2}$, I Clemente

'Sydney Children's Hospital and University of New South Wales, Sydney, Australia; ${ }^{2}$ IWK Health Centre, Dalhousie University, Halifax, Nova Scotia

Linguists have recognized that through different processes of meaning extension, such as metaphor, speakers often talk about their emotional experiences as involving their internal organs and external body parts (Enfield \& Wierzbicka, 2002). The results of a survey conducted by Piira, Grégoire and Clemente, assessing expressions of social pain in over 25 languages, will be presented. Respondents were requested for expressions of social pain that used physical terminology. Expressions were found in every language surveyed, "Broken Heart" being the most frequent one. Over the last decade there have been important advances in neuroimaging technology, resulting in further evidence of the close link between social and physical pain. Functional MRI techniques have demonstrated similarities in central neural circuitry involvement in the processing of both social and physical pain. The anterior cingulate cortex (ACC), the right ventral prefrontal cortex (RVPFC) and periaqueductal gray (PAG) have been identified as the most important structures. Physiological neuroendocrine systems, especially the opioid and the oxytocin systems, have also been shown to respond in a similar way to situations of physical and social pain in animals.

Progress in imaging and physiology is explaining what was thought to be a simple English language idiosyncrasy: being broken-hearted might mean much more to the brain than a simple twist of vocabulary, or the use of a metaphor. The presence of social pain expression using physical terminology in languages around the world is not a simple coincidence. Those similarities will be reviewed and discussed with workshop participants.

\subsection{4 .2}

\section{SOCIAL PAIN THEORY AND WHAT WE CAN LEARN FROM THE HURT OF SOCIAL EXCLUSION}

\section{Piira ${ }^{1}$, M-C Grégoire ${ }^{2}$}

${ }^{1}$ Sydney Children's Hospital and University of New South Wales, Sydney, Australia; ${ }^{2}$ IWK Health Centre, Dalhousie University, Halifax, Nova Scotia

Rejection and social exclusion are aversive social forces, typically directed at individuals who differ from the norm (esp, with regard to high aggressiveness or social withdrawal). It has been argued that such social forces serve to deter deviance from group norms, and may be experienced by the individual in a way that is analogous to a child learning that hot stoves should be avoided. 


\section{Abstracts}

According to an evolutionary framework, painful feelings have come to be associated with cues that are predictive of social exclusion experiences because evolutionary pressures resulted in social inclusion being critical to survival (MacDonald \& Leary, 2005). The social pain theory holds that the pain system has two important mechanisms that help in the response to threats of exclusion: (i) pain promotes a quick reaction in response to threat, and (ii) pain serves as a source of punishment to teach organisms to avoid threatening stimuli. Evidence from nonhuman animals as well as human children will be considered.

The association between the experience of rejection and pain sensitivity will be reviewed for human and nonhuman animals. There is some evidence to suggest that social threat may result in analgesic responses (MacDonald, Kingsbury \& Shaw, 2005). Results may be interpreted in terms of fight or flight responses.

Human facial, behavioural and physiological responses to social pain and physical pain will be discussed, and consideration given to how these responses impact on others within the social context.

Although a lot of the research in this field has been with adults, issues regarding social exclusion and bullying for children will be considered. Areas for further research with children will be identified.

\section{YOUNG INVESTIGATOR AWARD ABSTRACT}

\subsection{1}

\section{A PAIN-FREE WORLD FOR CHILDREN: ONE TRIAL AT A}

\section{TIME}

\section{A Taddio}

Scientist and Clinical Pharmacist, The Hospital for Sick Children, and Assistant Professor, Faculty of Pharmacy, University of Toronto, Toronto, Ontario

The last twenty years has seen a surge in research on procedural pain in infants and children. This has been due to many factors, including: the development of new analgesics, the growing reliability in our ability to assess pain, and the corresponding concerns for the effects of untreated pain. This presentation will focus on clinical trials performed by our centre in infants and children undergoing procedural pain, the gains we have made as a result of these studies and the lessons we have learned. 


\section{Biology, Neurobiology, Physiology, Anatomy}

\section{A1}

\section{LONG-TERM ALTERATIONS IN PAIN SENSITIVITY 8 YEARS FOLLOWING NEONATAL SURGERY}

$\underline{\text { R Schouw }}^{1}$, H Duivenvoorden ${ }^{1}$, D Tibboel ${ }^{1}$, K Anand $^{2}$, J Peters ${ }^{1}$

'Erasmus MC - Sophia Children's Hospital, Rotterdam,

The Netherlands; ${ }^{2}$ University of Arkansas for Medical Sciences, Little Rock, Arkansas, USA

OBJECTIVE: The aim of this study was to assess whether children who underwent major surgery within the first 3 months of life were more sensitive to pain later in life in comparison with age- and gender-matched controls. DESIGN: A prospective case-control study was conducted. After undergoing major surgery within the first three months of life, 58 infants were followed up to the age of 6-11 years (mean age 7.8 years). After this period, children's detection thresholds for warmth and cold as well as their pain thresholds for heat and cold were assessed using Quantitative Sensory testing. A thermode $(3 \times 3 \mathrm{~cm})$ was subsequently attached to the thenar eminence of the hand, the ipsilateral and contralateral side of the wound. At every location, the temperature of thermode was steadily increased/decreased at a rate of $1^{\circ} \mathrm{C} / \mathrm{sec}$. When the children perceived a particular sensation they were instructed to press a button to terminate the test. Findings were compared with age- and gender-matched controls. RESULTS: Former patients were significantly less sensitive to cold and warmth perception at the ipsilateral side of the wound $(\mathrm{P}<0.05)$, but not at the thenar eminence or the contralateral side of the wound. Controversely, former patients were significantly more sensitive to heat and cold pain both at the ipsilateral side as well as the contralateral side of the wound $(\mathrm{P}<0.05$ for heat and $\mathrm{P}<0.01$ for cold); pain thresholds on the thenar eminence did not differ with that of the controls.

CONCLUSION: Surgery during early life, in spite of adequate analgesia, leads to an altered sensitivity in the dermatome of injury, which may last for up to 11 years after the initial surgical tissue damage. These long-term effects of early pain exposure may result from a combination of peripheral/spinal and supraspinal changes.

\section{A2}

\section{LONG-TERM CONSEQUENCES OF EARLY INFANT INJURY MORE PRONOUNCED IN FORMER PRETERM BORNS}

R Schouw $^{1}$, H Duivenvoorden ${ }^{1}$, D Tibboel ${ }^{1}$, K Anand $^{2}$, J Peters ${ }^{1}$

${ }^{1}$ Erasmus MC - Sophia Children's Hospital, Rotterdam,

The Netherlands; ${ }^{2}$ University of Arkansas for Medical Sciences,

Little Rock, Arkansas, USA

AIM: Repeated exposure to pain stimuli on the short-term induces sensitization in preterm-born neonates, whereas in term-born neonates it leads to habituation. On the long-term prolonged hypersensitivity develops in both groups, extending beyond the period associated with complete healing of the initial tissue injury. It is not clear whether these long-term effects are more pronounced in the preterm-born neonates. This study, therefore, was conducted to investigate whether long-term consequences of neonatal pain exposure upon somatosensory processing differ between prematureborn and term born-neonates.

DESIGN: A prospective case-control study was conducted. After undergoing artificial ventilation during the first weeks of life, 53 children were followed up to 6-11 years (mean age 8.6 years). After this period, children's detection thresholds for warmth and cold as well as their pain thresholds were assessed using Quantitative Sensory testing. A thermode $(3 \times 3 \mathrm{~cm})$ was subsequently attached to the thenar eminence of the hand and the heel. At both locations, the temperature of thermode was steadily increased/decreased at a rate of $1^{\circ} \mathrm{C} / \mathrm{sec}$. When a specified sensation was first perceived, the children were instructed to press a button to terminate the test. Findings were compared with age- and gender-matched controls. RESULTS: Former patients were significantly less sensitive to cold and warmth perception on the heel $(\mathrm{P}<0.05)$, but not on the hand. Conversely, former patients were significantly more sensitive to heat and cold pain on the heel $(\mathrm{P}<0.05)$; pain thresholds on the hand did not differ with controls. Preterm-born neonates were more hypo- and hypersensitive than term-born patients.

CONCLUSION: Eight years following neonatal Intensive Care Unit admission, alterations in pain processing at the area of previous tissue damage are still measurable. In accordance to experimental findings in animals, these long-term consequences are more prominent in pretermborn neonates, confirming the existence of a developmental window.

\section{A3 QUANTITATIVE SENSORY TESTING IN CHILDREN WITH CHRONIC PAIN}

P McGrath ${ }^{1,2}$, S Brown ${ }^{3}$, A Mincic ${ }^{4}$, A Lisoway ${ }^{3}$, C Kogan ${ }^{3}$, T Williams $^{3}$, K Davis ${ }^{4}$

${ }^{1}$ IWK Health Centre, Halifax, Nova Scotia; ${ }^{2}$ The University of Toronto; ${ }^{3}$ The Hospital for Sick Children; ${ }^{4}$ Toronto Western Research Institute, Toronto, Ontario

OBJECTIVE: Little is known concerning the neurophysiological abnormalities underlying pediatric chronic pain. To gain insight into the function of tactile and thermal signalling pathways we have characterized sensory deficits in children with chronic pain.

METHODS: Quantitative Sensory Testing (QST) was conducted on 19 children diagnosed with chronic pain (primarily Complex Regional Pain Syndrome - Type 1, CRPS-1, and Pain and Associated Disability Syndrome, PADS; $10 \mathrm{~m}$, 9f; mean age $13.9 \mathrm{yrs})$ and a pain-free comparison cohort of 37 healthy controls (18m, 19f; mean age $15.3 \mathrm{yrs})$. QST included determinations of detection and pain thresholds (method of limits) to tactile and thermal stimuli and suprathreshold thermal testing to establish stimulus-response curves. Stimuli were applied to the volar surface of the forearms, and additionally for pain patients at the painful and homologous contralateral body sites (data on painful sites not reported here). Numerical rating scales were used to assess pain intensity.

RESULTS: Analyses of variance indicated that there were no significant differences in tactile and thermal thresholds between the patient and healthy control group. Similarly, there were no significant differences by sex although the mean cold pain threshold for girls was slightly lower than that for boys. Mean pain thresholds were $291.7 \mathrm{mN}$ (SD 167) for tactile stimulation, $40.4^{\circ} \mathrm{C}(\mathrm{SD} 4.2)$ for heat pain, and $18.5^{\circ} \mathrm{C}$ (SD 10.3) for cold pain. Consistent with data in adults, S-R curves were best described by power functions. Interestingly, pain ratings tended to be higher in the chronic pain patient group than the healthy cohort.

CONCLUSION: QST is a feasible clinical tool for use with children and adolescents. Preliminary analyses indicate that children with CRPS-1 and PADS do not have generalized sensory threshold abnormalities, although there may be subtle abnormalities in suprathreshold pain perception. These data highlight the importance of suprathreshold pain testing.

\section{Neonatal}

\section{B1}

KANGAROO CARE FOR PROCEDURAL PAIN MANAGEMENT IN VERY PREMATURE NEONATES 28-32 WEEKS GESTATIONAL AGE

C Johnston ${ }^{1}$, F Filion ${ }^{1,2}$, L Bell ${ }^{3}$, M Campbell-Yeo ${ }^{1,4}$, K McNaughton ${ }^{1}$, C Walker ${ }^{1}$, A Nuyt ${ }^{2}$, A Finley ${ }^{4,5}$, C Goulet ${ }^{2}$ ${ }^{1}$ McGill University; ${ }^{2}$ University of Montréal, Montréal; ${ }^{3}$ University of Sherbrooke, Sherbrooke, Québec; ${ }^{4}$ IWK Children's Hospital; ${ }^{5}$ Dalhousie University, Halifax, Nova Scotia

AIM: Earlier studies have demonstrated the efficacy of maternal kangaroo care to decrease pain response and facilitate recovery from the frequent and invasive clinical procedure of heel lance for blood sampling in both full term and preterm neonates. One proposed mechanism for the efficacy of $\mathrm{KC}$ was endogenous opiate release during the skin-to-skin contact with the mother. Given that the endogenous opiate system is immature before 32 weeks, it was questionable whether $\mathrm{KC}$ with infants younger than 
32 weeks would diminish pain response. This study was therefore undertaken to determine if maternal kangaroo care could be efficacious in infants less than 32 weeks gestational age for the common painful procedure of heel lance.

METHODS: Infants born between the ages of 28-32 weeks gestational age and less than two weeks of life were studied in a randomized crossover trial to compare pain response and time to recovery following a heel lance. The Premature Infant Pain Profile, a composite measure of behavioural indicators of pain (facial actions) and physiological indicators of pain (heart rate, oxygen saturation) is the primary outcome.

RESULTS: A sample of 52 babies is sought and as of October 8, 2005, 48 babies have been recruited. Preliminary results on 14 infants show significant decrease in the behavioural indicator of pain, facial actions, by $20 \%$, a significant decrease in time to recover $(33 \%)$ and a nonsignificant trend in the physiological indicators favouring $\mathrm{KC}$.

CONCLUSIONS: Even premature neonates born before the last trimester of gestation may benefit from the analgesic effects of maternal kangaroo care.

\section{B2}

\section{ENHANCING KANGAROO CARE EFFICACY FOR MANAGEMENT OF PROCEDURAL PAIN IN PRETERM NEONATES 32-36 WEEKS GESTATIONAL AGE THROUGH MULTIPLE SENSORY MODALITIES}

C Johnston ${ }^{1}$, F Filion ${ }^{1,2}$, M Campbell-Yeo ${ }^{1,4}$, L Bell ${ }^{3}$,

K McNaughton ${ }^{1}$, C Walker ${ }^{1}$, A Nuyt ${ }^{2}$, C Coulet $^{2}$, A Finley ${ }^{4,5}$

${ }^{1}$ McGill University; ${ }^{2}$ University of Montréal, Montréal;

${ }^{3}$ University of Sherbrooke, Sherbrooke, Québec; ${ }^{4}$ IWK Childrens

Hospital; ${ }^{5}$ Dalhousie University, Halifax, Nova Scotia;

AIM: Earlier studies have demonstrated the efficacy of maternal kangaroo care to decrease pain response and facilitate recovery from the frequent and invasive clinical procedure of heel lance for blood sampling in both full term and preterm neonates. It is thought that tactile stimulation is the primary modality in which kangaroo care works. The purpose of this study is to determine if the addition of vestibular stimulation (rocking), auditory stimulation (talking, singing), and gustatory stimulation (sucking) would enhance the efficacy of kangaroo care.

METHODS: Infants born between the ages of 32-36 weeks gestational age and less than two weeks of life were studied in a randomized crossover trial to compare pain response and time to recovery following a heel lance. The Premature Infant Pain Profile, a composite measure of behavioural indicators of pain (facial actions) and physiological indicators of pain (heart rate, oxygen saturation) is the primary outcome.

RESULTS: A required sample of 64 babies has been recruited. Preliminary results on 21 infants shows no differences between usual KC and enhanced $\mathrm{KC}$.

CONCLUSIONS: The efficacy of kangaroo care in preterm neonates may be predominantly through touch; additional stimulation by the mother may not provide additional benefit.

\section{B3}

\section{EFFICACY AND SAFETY OF CONTINUOUS INFUSION OF KETOROLAC IN NEONATES AND INFANTS}

\section{$\underline{\text { D Galante, }} \underline{\mathrm{G} \text { Pellico }}$}

Department of Anesthesia and Intensive Care, University Hospital "Ospedali Riuniti", Foggia, Italy

BACKGROUND: Ketorolac tromethamine is a nonsteroidal antiinflammatory analgesic (NSAID). The mode of action is through inhibition of prostaglandin synthesis. Ketorolac may reduce opioid use and prevent opioid-associated side effects that are frequent in neonates and infants. The aim of our study was to evaluate its safety and analgesic efficacy in this age group.

METHODS: 16 infants ASA I, aged 15 days-6 months undergoing genitourinary, abdominal and thoracic surgery received a continuous infusion of ketorolac $0.05 \mathrm{mg} / \mathrm{kg} / \mathrm{h}$ plus ranitidine $(30 \mathrm{mcg} / \mathrm{kg} / \mathrm{h}$ within the first month of life, $200 \mathrm{mcg} / \mathrm{kg} / \mathrm{h}$ for $1-6$ months age group) for 48 hours by elastomeric pump at $2 \mathrm{~mL} /$ hour for postoperative pain management.
Neonates and infants also received intravenous ketorolac $1 \mathrm{mg} / \mathrm{kg}$ as preemptive analgesia. Induction was followed by balanced anesthesia with sevoflurane, Air/O2 mixture, cisatracurium, fentanyl or continuous infusion of remifentanil. The beginning of ketorolac infusion was performed 30 minutes before the end of the operation. Heart rate, systolic and diastolic arterial blood pressure, SpO2, RR, ETCO2 were monitored. Full blood tests, including coagulation tests, were performed to $24 \mathrm{~h}$ and $48 \mathrm{~h}$ after ketorolac administration. Postoperative analgesia was evaluated with Neonatal Infant Pain Scale (NIPS).

RESULTS: All patients in our study had adequate analgesia and did not require additional drugs. Heart rate, ECG, systolic and diastolic arterial blood pressure, $\mathrm{SpO} 2, \mathrm{RR}$ and urinary output were all within the normal ranges. No respiratory depression was reported. Monitoring of hematological parameters showed no significant changes. There was no bleeding from nasogastric tubes, surgical wounds and blood withdrawal sites. None of the patients complained of postoperative nausea or vomiting.

DISCUSSION: A few studies indicate that ketorolac may increase bleeding more so than other NSAIDs, but the evidence is conflicting. Severe adverse effects of NSAIDs in children are very rare. Literature is scarce with regard to their use in neonates and infants. Ketorolac is approved by the Food and Drug Administration for parenteral administration and is a therapeutic alternative to opioids. Opioids are frequently associated with respiratory depression, paralytic ileus and urinary retention. The ketorolac dosage used in our study is correlated with the higher volume of distribution in neonates.

CONCLUSIONS: Continuous infusion of ketorolac is a valid alternative to opioids for pain therapy and can be used to treat pain without an increased risk of bleeding complications. In association with regional anesthesia (eg, lumbar/thoracic epidural anesthesia or caudal block) may constitute a safe and efficacy technique for postoperative pain therapy in neonates and infants.

\section{REFERENCES}

Papacci P, De Francisci G, Iacobucci T, et al. Use of intravenous ketorolac in the neonate and premature infant. Paediatr Anaesth 2004;14:487-92.

Chauhan RD, Idom CB, Noe HN. Safety of ketorolac in the pediatric population after ureteroneocystostomy. J Urol 2001;166:1873-5.

Moreno M, Castejon FJ, Palacio MA. Patient-controlled analgesia with ketorolac in pediatric surgery. J Physiol Biochem 2000;56:209-16.

Buck ML. Clinical experience with ketorolac in children. Ann Pharmacother 1994;28:1009-13.

\section{B4}

\section{SUCROSE REDUCES PAIN DURING INTRAMUSCULAR VITAMIN K INJECTION IN NEWBORNS}

R Smith ${ }^{1}$, V Shah ${ }^{2}$, J Katz ${ }^{3}$, E Darra ${ }^{1}$, A Taddio ${ }^{1}$

${ }^{1}$ Department of Population Health Sciences, The Hospital for Sick Children; ${ }^{2}$ Department of Paediatrics, Mount Sinai Hospital; ${ }^{3}$ Department of Psychology, York University, Toronto, Ontario

BACKGROUND: Sucrose reduces pain in young infants undergoing tissue-damaging cutaneous procedures such as venipuncture and heel lance. Studies of the effectiveness of sucrose analgesia for intramuscular injection of vitamin $\mathrm{K}$ in the newborn, however, are lacking. Since all newborns undergo vitamin $\mathrm{K}$ injection, demonstrating the effectiveness of sucrose analgesia has implications for the management of pain for the entire neonatal population.

OBJECTIVE: To determine the analgesic effectiveness of sucrose for intramuscular injection of vitamin $\mathrm{K}$ in newborns.

DESIGN/METHODS: Double-blind randomized controlled trial in healthy full term neonates. Neonates received either sucrose or water on the tongue two minutes prior to vitamin $\mathrm{K}$ injection. Pain was assessed from videotapes of participating infants using facial grimacing (FG; brow bulge, eyes squeezed shut and nasolabial furrow) and cry duration (CD). RESULTS: One hundred and nineteen infants participated. There were no significant differences in infant characteristics between groups; mean (SD) gestational age and birthweight was 39 weeks (1 week) and $3.4 \mathrm{~kg}$ 
$(0.4 \mathrm{~kg})$, respectively for both the sucrose and water groups. Repeated measures ANCOVA revealed a statistically significant main effect of treatment group for facial grimacing $(\mathrm{P}=0.018)$ and for cry duration $(\mathrm{P}=0.028)$, a significant main effect of procedure phase for both FG and CD (both $\mathrm{P}<0.001$ ), and no significant group $\times$ phase interaction for either FG or CD. Overall, the sucrose group showed $19 \%$ less facial grimacing and a $28 \%$ reduction in crying time than the water group.

CONCLUSIONS: Sucrose reduced pain during intramuscular injection of vitamin $\mathrm{K}$ in newborn infants and is recommended for routine use in clinical practice.

\section{B5} NEONATAL FACIAL CODING SCALE SCORES AND
SPECTRAL CHARACTERISTICS OF INFANT CRYING
DURING NEWBORN CIRCUMCISION

V Tutag Lehr ${ }^{1}$, P Zeskind ${ }^{2}$, J Ofenstein ${ }^{1}$, J Aranda ${ }^{1}$

${ }^{1}$ Clinical Pharmacology and Pediatric Pharmacology Research Unit Network, Children's Hospital of Michigan, Wayne State University, Detroit, Michigan; ${ }^{2}$ Department of Pediatrics, Carolinas Medical Center, Charlotte, North Carolina, USA

OBJECTIVE: To determine the relation between Neonatal Facial Coding System (NFCS) scores and measures of infant crying during newborn circumcision.

METHODS: Relations among measures of infant crying and facial activity were tested during the lysis procedure of circumcision in 44 healthy term males ( $<3$ days of age) using three analgesic interventions. Video and audio recordings of the entirety of lysis provided the basis for coding facial activity for NFCS scores and spectral analysis of cry sounds for measures of crying, respectively. Pearson product moment correlations, principal component factor analyses, and stepwise linear regressions examined relations between NFCS scores and measures of infant crying.

RESULTS: Significant correlations were found between higher NFCS scores and higher amplitudes of crying at the Peak fundamental frequency, Dominant Frequency and overall cry sample $(\mathrm{P}<0.01)$ and a lower Peak fundamental frequency $(\mathrm{P}<0.01)$. Factor analysis showed three significant orthogonal dimensions underlying measures of infant crying accounting for $42.3 \%, 17.8 \%$ and $14.6 \%$ of the variance, respectively. Regression analysis showed that the factor describing all three factors accounted for significant and separate portions of the variance $(\mathrm{P}<0.001)$ with the best prediction being the factor comprised by measures describing the amplitude and rapidity of the cry sound. Significant predictors of NFCS scores were amplitude and rapidity of cry sound $(\mathrm{P}<0.002)$, greater turbulence and louder sounds at higher frequencies $(\mathrm{P}<0.002)$, and frequency characteristics $(\mathrm{P}<0.007)$.

CONCLUSION: This study provides some of the first known evidence linking specific measures of infant crying with an independent, validated measure of pain.

\section{B6}

NEONATAL PAIN, STRESS AND COGNITIVE DEVELOPMENT IN PRETERM INFANTS

$\underline{M ~ T u}^{1,2}$, Grunau $^{1,2}$, J Petrie-Thomas ${ }^{2}$, J Weinberg ${ }^{2,3}$,

M Whitfield ${ }^{1,2}$

${ }^{1}$ Centre for Community Child Health Research; ${ }^{2}$ Pediatrics;

${ }^{3}$ Cellular and Physiological Sciences, University of British

Columbia, Vancouver, British Columbia

BACKGROUND: In infants born at very low gestational age (VLGA $<32 \mathrm{wks}$ ), stress of repeated procedures in the neonatal intensive care unit (NICU), may contribute to altered neurobehaviour. Moreover, maternal caregiving factors may either compound or ameliorate outcomes.

OBJECTIVE: To assess whether maternal factors (stress, interactive behaviour) buffer relationships between cumulative neonatal pain, infant basal cortisol, and neurodevelopment (as indexed by focused visual attention) at 8 mo corrected age (CA).

METHODS: 83 VLGA (42 boys, 41 girls; mean GA 29.4, sd 2.7 wks) recruited in the level III NICU, and 42 full term ( 24 boys, 18 girls; mean GA 40.1 sd 1.0) infants, from the normal newborn nursery,
Children's Women's Health Centre of BC were seen at 8 mo CA. Variables were: neonatal pain exposure (number of skin breaking procedures from birth to term, from medical chart review); maternal stress (Parenting Stress Index); video scoring of both maternal interactive behaviour (during mother-infant free play) and infant focused attention (during toy exploration); and infant basal salivary cortisol.

RESULTS: VLGA infants had 17 to 441 skin breaking procedures. Using hierarchical regression, higher neonatal pain exposure (adjusted for SNAP-II and iv morphine) was related to poorer infant attention $(\mathrm{P}=0.017)$. However, higher maternal interaction behaviour partially buffered this adverse effect as long as mothers reported low stress $(\mathrm{P}=0.024)$. In infants exposed to a combination of high maternal stress and high interactive behaviours, higher infant basal cortisol was related to poorer focused attention $(\mathrm{P}=0.040)$.

CONCLUSIONS: High maternal stress appears to compound the adverse effects of neonatal pain exposure and high basal cortisol on focused attention in preterm infants. We are currently addressing these questions at 18 months.

Support: NICHD (HD39783) and Human Early Learning Partnership (HELP, Ministry of Children and Family Development, Government of British Columbia)

\section{B7}

\section{LOCAL ANESTHESIA VIA SUBCUTANEOUS CATHETER AFTER THORACOTOMY IN PREMATURE INFANTS} M Bartocci ${ }^{1}$, S Lundeberg ${ }^{2}$

${ }^{1}$ Neonatal Intensive Care Unit; ${ }^{2}$ Acute Pain Treatment Service, Astrid Lindgren Children's Hospital, Karolinska University Hospital, Stockholm, Sweden

BACKGROUND: All interventions requiring thoracotomy such as ductus ligation or esophageal repair, are characterized by a postoperative period where the control of pain is of utmost importance. This type of operation is mainly performed during the neonatal period and often in premature newborn infants (ie, when the brain is under vulnerable development). Opioids are the most common kind of analgesics used in the postoperative phase in newborn infants. Recent studies have shown both the detrimental effects that pain itself and the potential harmful consequences that morphine may have on brain development especially in preterm newborn. Besides, opioid treatment is often accompanied by side effects such as respiratory depression which prolongs the ventilator treatment postoperatively. Thus it urges to find new alternative treatments that allow pain relief as well as lesser use of opioids.

The main aim of the study was to assess if a local anesthetic such as L-bupivacaine in repeated doses by a wound catheter may reduce the need of morphine and accelerate the weaning from the ventilator.

METHODS: We retrospectively analyzed data of 21 newborns infants (group A 10 infants, group B 11 patients) who underwent thoracotomy after ductus ligation or repair of esophageal atresia. Theese operations require a similar surgical approach. At the end of the operation (group B) one or two subcutaneous catheters (Portex ${ }^{\circledR}$ epidural catheters with two side holes) were inserted under the fascia muscularis before skin closure. The catheters were filled with med L-bupivacaine (Chirocaine ${ }^{\circledR}$ $1.25 \mathrm{mg} / \mathrm{mL}$ ) and a dose of $2 \mathrm{mg} / \mathrm{kg}$ was infused subcutaneously 4 times daily. Morphine infusion at a starting dose of $10 \mathrm{microg} / \mathrm{kg} / \mathrm{h}$ and paracetamol (Perfalgan ${ }^{\circledR} 7.5 \mathrm{mg} / \mathrm{kg} 4$ times daily) were administered iv in both groups $(A \& B)$. Pain assessment was performed with Astrid Lindgren Children's Hospital Pain Scale on a regular basis (minimum every 3 hour). The morphine infusion was increased or decreased accordingly.

RESULTS: 10 children were treated according to the standard protocol (morphine + paracetamol, group A) and 11 infants were additionally treated with local anesthesia (L-bupivacaine, group B). Mean weight on the operating day was $1181 \mathrm{~g}$ and mean postnatal age was 29.6 weeks. The wound catheters were maintained in place for a mean period of 4 days. No side effects have been reported. The hemodynamic parameters (HR, BP and $\mathrm{FiO} 2$ ) did not show any significant difference. The need for morphine infusion was significantly shorter in group B (1.8 vs 4.5 days). Total morphine consumption was significantly lower in group B (274 vs $641 \mathrm{microg} / \mathrm{kg}$ ) 
CONCLUSION: We have safely used a local anesthetic drug such as L-bupivacaine for postoperative pain treatment after thoracotomy in premature neonates. In our study, despite the limited study population, we have shown that this promising approach might reduce the need of opioids in the postoperative phase and the side effects of the drug.

\section{B8}

\section{RANDOMIZED CONTROLLED TRIAL OF AMETHOCAINE GEL FOR INTRAMUSCULAR INJECTION IN TERM NEONATES}

V Shah $^{1,3}$, A Taddio ${ }^{2,3}$, L Noble', P Shah ${ }^{1,3}$, R Hancock ${ }^{2}$, A Ohlsson 1,3

${ }^{1}$ Mount Sinai Hospital; ${ }^{2}$ Hospital for Sick Children;

${ }^{3}$ University of Toronto, Toronto, Ontario

BACKGROUND: Most neonates are subjected to injection of vitamin K intramuscularly after birth. Even though it is a painful procedure, pain relieving interventions are not routinely administered. To date, there have been no studies that have evaluated analgesics for this procedure.

OBJECTIVE: To assess the analgesic effectiveness of amethocaine gel for intramuscular injection of vitamin $\mathrm{K}$ in term neonates.

DESIGN/METHODS: We performed a double-blind randomized controlled trial of amethocaine gel $(1 \mathrm{~g})$ compared to placebo in term neonates prior to intramuscular injection of vitamin K. Neonates were videotaped during the injection and for 20 seconds after the injection (recovery). Pain was assessed using percent facial grimacing (brow bulge, eye squeeze and nasolabial fold) and percent time spent crying. Local reactions were recorded. To demonstrate a $20 \%$ reduction in the facial grimacing pain scores during injection between groups (beta $=0.8$, alpha $=0.05), 98$ neonates needed to be enrolled.

RESULTS: One hundred and ten neonates were enrolled of whom 3 were excluded as the injection was given prior to videotaping. The mean (SD) gestational age was 39.3 weeks (1.1) vs $39.2(1.0),(P=0.55)$, and birth weight was $3543 \mathrm{~g}(406)$ vs $3405 \mathrm{~g}(352),(\mathrm{P}=0.06)$, for amethocaine and placebo groups respectively. The mean (SD) percent facial grimacing score during the injection and recovery phases were $69 \%(34 \%)$ vs $76 \%(34 \%)$, $(\mathrm{P}=0.3)$ and $79 \%(24 \%)$ vs $76 \%(29 \%),(\mathrm{P}=0.6)$, for amethocaine and placebo groups respectively. The percent time spent crying during the injection and recovery phases were $54 \%(35 \%)$ vs $62 \%(38 \%),(P=0.26)$, and $63 \%(33 \%)$ vs $70 \%(34 \%),(\mathrm{P}=0.32)$, for the amethocaine and placebo groups respectively. Transient local skin reactions (mild redness or blanching) were seen in $16 \%$ of neonates who received amethocaine compared to $7 \%$ who received placebo ( $\mathrm{P}=0.36$ ).

CONCLUSIONS: Amethocaine gel was ineffective in reducing pain associated with intramuscular injection of vitamin $\mathrm{K}$ in term neonates. No serious adverse events were observed. Amethocaine cannot be recommended for analgesia for intramuscular injection of vitamin $\mathrm{K}$ in neonates. Study funded by: Striving for Excellence Fund, Mount Sinai Hospital

\section{B9}

\section{PAIN MANAGEMENT PRACTICES DURING MINOR PAINFUL PROCEDURES IN A COHORT OF INFANTS WITH A PROLONGED LENGTH OF STAY}

D Harrison $^{1,2,3,4}$, P Loughnan ${ }^{2,3}$, E Manias ${ }^{1}$, L Johnston ${ }^{1,3,4}$ ${ }^{1}$ School of Nursing; ${ }^{2}$ Department of Paediatrics, The University of Melbourne; ${ }^{3}$ Department of Neonatology, Royal Children's Hospital; ${ }^{4}$ Critical Care \& Neurosciences, Murdoch Childrens Research Institute, Melbourne, Victoria, Australia

AIM: To ascertain pain management practices during minor painful procedures in a cohort of infants admitted to a NICU with an expected length of stay $>28$ days.

METHODS: A prospective longitudinal observational study undertaken in the Neonatal Unit, Royal Children's Hospital. Melbourne. Data relating to minor painful procedures and analgesia and sedation administration were collected on a daily basis.

RESULTS: Fifty-five infants met the inclusion criteria. Preliminary analysis of results for 40 infants, 16 female and 24 male, with a mean length of stay of 59 (SD 28) days is presented. Mean gestational age on admission was 35 weeks (SD 5). Six infants (15\%) died prior to discharge. A total of 2618 minor procedures were recorded for the 40 infants during the course of their hospitalization. The majority were heel lances (HL) with a total of 1880 HLs recorded, $72 \%$ of all minor procedures.

There were 381 venepuncture/intravenous line insertion procedures recorded ( $14 \%$ of all minor procedures). There were 84 intramuscular or subcutaneous injections, 68 arterial line insertions/arterial stabs and 34 eye examinations recorded. There were 157 'other' minor procedures recorded, of which 54 were skin-breaking procedures.

The majority of all procedures were successfully completed during the first attempt.

Of the 2618 procedures in total documented, oral sucrose use was recorded during 1488 (57\%) of these procedures. Of the remaining 1130 procedures in which sucrose was not documented, some form of opioid analgesia was given within the previous 24 hours for 749 of these procedures $(66 \%)$. Therefore there were 381 procedures $(14 \%)$ in which either no sucrose or no other analgesia had been given in the previous 24 hours.

CONCLUSIONS: This cohort of infants with a prolonged hospitalization was exposed to a large number of minor skin-breaking procedures over the course of their hospitalization. Although some form of analgesia was used for the majority of procedures, $14 \%$ were performed with no analgesia.

\section{B10}

\section{ANALGESIC AND SEDATIVE ADMINISTRATION IN A COHORT OF INFANTS WITH A PROLONGED LENGTH OF STAY}

D Harrison ${ }^{1,2,3,4}$, P Loughnan ${ }^{2,3}$, E Manias ${ }^{1}$, L Johnston 1,3,4

${ }^{1}$ School of Nursing, ${ }^{2}$ Department of Paediatrics, The University of Melbourne; 'Department of Neonatology, Royal Children's Hospital; ${ }^{4}$ Critical Care \& Neurosciences, Murdoch Childrens Research Institute, Melbourne, Victoria, Australia

AIM: To ascertain analgesic and sedative administration in a cohort of infants with an expected length of stay in a NICU of $>28$ days.

METHODS: A prospective longitudinal observational study.

SETTING AND PARTICIPANTS: A cohort of infants admitted to the Neonatal Unit, Royal Children's Hospital, Melbourne with a predicted length of stay $>28$ days.

Data relating to both minor and major painful procedures, analgesic and sedative administration and ventilation status were collected on a daily basis.

RESULTS: 55 infants met the inclusion criteria. Preliminary analysis of results for 40 infants, 16 female and 24 male, with a mean length of stay of 59 (SD 28) days is presented. Mean gestational age (GA) on admission was 35 weeks (SD 5). Six infants (15\%) died prior to discharge. Thirty-five infants (87\%) required assisted ventilation and all 40 infants underwent surgery during their hospitalization.

During their hospitalization, 35 of the 40 infants $(87 \%)$ received morphine. For the five infants who received no morphine, all were administered codeine. Thirty-two infants (80\%) received sedatives; either oral chloral hydrate or intravenous midazolam.

Only two infants in this cohort did not receive any paracetamol during the course of their hospitalization. For the remaining 38 infants, paracetamol was administered on $32 \%$ of hospital days. All 40 infants were administered sucrose for procedural-related pain during their hospitalization.

Although 30 of the 40 infants had pain scores documented at some stage during their hospitalization, the frequency of documentation was extremely low, with pain scores being documented on only 133 hospital days in total (5\%).

CONCLUSIONS: Analgesic and sedative use in this cohort of infants with a length of stay $>28$ days, and complex health needs is substantial. All infants received opioid analgesics, and 38 of the 40 infants were administered paracetamol during the hospitalization. Despite the extensive use of analgesics, pain assessment scores were infrequently documented. 


\section{B11}

\section{PAIN AND DISTRESS RESPONSES IN PRETERM NEONATES UNDER REPEATED DOSES OF SUCROSE SCHEDULE FOR PAIN RELIEF}

$\underline{M}$ Linhares, C Gaspardo, C Miyase, J Chimello, V Klein, T Cugler, F Martinez

Faculdade de Medicina de Ribeirão Preto da Universidade de São Paulo, Ribeirão Preto, Brazil

OBJECTIVE: To examine the efficacy of repeated doses of oral sucrose for pain relief during painful procedures in NICU through assessing pain and distress responses of preterm neonates.

METHOD: The randomized double blind study included 33 preterm very low birth-weight neonates; allocated into two groups: GS - Sucrose Group $(\mathrm{n}=17)$ and GC - Control Group $(\mathrm{n}=16)$. The GS received oral sucrose $(25 \% ; 0.5 \mathrm{~mL} / \mathrm{Kg}$ ) and the GC received sterile water, both groups $2 \mathrm{~min}$ before each invasive painful procedure during three days. The neonates' responses of pain and distress were assessed during blood collection in four consecutive days. In the 1st assessment, the neonates of both groups received no solution before the painful procedures in NICU. In the next three assessments (2nd, 3rd, 4th) the GS were under repeated doses of sucrose schedule and the GC were receiving water. Each assessment was divided into five phases: Baseline (BL), Preparation for Puncture (PP) Puncture (P), Recovery with handling (RH), and Recovery (R). The facial actions (NFCS), heart rate, and behavioural state (sleep-awake) were assessed, using cut-off scores of painful and distress responses. The neonates' medical charts were reviewed.

RESULTS: The GS showed significantly fewer neonates with facial actions signs of pain (NFCS > 3) than GC in P (2nd) and PP (3rd). The GS showed significantly fewer neonates with awake state than GC in $P$ (2nd, 4th). The GS showed significantly fewer neonates crying during PP (2nd), P (2nd, 4th) and RH (3rd). There was no difference between groups in heart rate measure; in both groups, at least $50 \%$ of the neonates showed heart rate $<160$ in $\mathrm{P}$ and $\mathrm{RH}$ (2nd, 3rd, 4th). No clinical adverse effects were found in neonates' health status.

CONCLUSIONS: The efficacy of sucrose was maintained for pain relief and regulation of behavioural responses, but not for physiological ones, in preterm neonates during painful procedures in NICU.

Acknowledgements: Supported by National Council of Research (CNPq) and Foundation for Research Support of São Paulo (FAPESP).

\section{B12}

\section{DIFFERENCES IN THE ASSESSMENT OF THE INTENSITY OF PROCEDURAL PAIN IN NICUS}

E Cignacco $^{1}$, J Hamers ${ }^{2}$, L Stoffel ${ }^{3}$, N Schütz ${ }^{4}$, R van Lingen ${ }^{5}$, L Zimmermann ${ }^{6}$, M Nelle $^{3}$

${ }^{1}$ Women's Clinic, University Hospital Insel, Bern, Switzerland; ${ }^{2}$ University of Maastricht, Section Nursing Science, Maastricht, The Netherlands; ${ }^{3}$ Children's Clinic, University Hospital Insel, Bern; ${ }^{4}$ Children's University Hospital, Zürich, Switzerland; ${ }^{5}$ Isala Clinics, Princess Amalia Department of Paediatrics Division of Neonatology, Zwolle; ${ }^{6}$ University of Maastricht, Neonatology, Department of Paediatrics, Maastricht, The Netherlands

BACKGROUND: Despite an increased awareness among clinicians regarding pain management in a NICU, pain associated with routine procedures is often not adequately managed. Barriers to pain management are related to nurses' and physicians' knowledge and experience and the challenges of collaborative decision-making. Due to a probably different perception of the pain intensity of procedures, this decision process may be often conflicting.

AIMS: To gain an insight into nurses' and physicians' assessment of pain intensity of routine procedures.

METHODS: A query with 431 neonatal health care providers from 4 tertiary level NICUS has been performed for the subjective pain assessment of 27 different procedures. Each procedure was assessed on a $10 \mathrm{~cm}$ Visual Analogue Scale (cut-off for painful procedures 4) under the assumption that there was no analgesia given.

RESULTS: Using MANOVA a significant difference between nurses' and physicians' pain intensity ratings was found $(\mathrm{P}<0.05)$. Nurses are assessing procedures as more painful. The mean VAS ratings for the insertion of $a$ thoracal drain (mean physicians 8.00/nurses 9.09), intubation (mean physicians 7.04/nurses 8.25) and extubation (mean physicians 3.24/nurses 4.68) were significantly different $(\mathrm{P}<0.01)$; the same holds true for "lumbar punction" (mean physicians 6.31/nurses 7.86), bladder punction (mean physicians 5.71/nurses 7.32), eye control (mean physicians 5.47/ nurses 6.93), insertion of an intravenous or intraarterial line (mean physicians 5.51/nurses 6.47) $(\mathrm{P}<0.05)$. Furthermore, there was an overall difference in ratings for the 4 participating NICUS (overall mean pain score: 4.59 C 5.23; $\mathrm{P}<0.01$ ) with the highest ratings for the NICU in Bern. CONCLUSIONS: Nurses rated all the procedures as more painful than physicians. This could be an explanation for the controversial decisionmaking regarding pain relieving interventions. The regular continuing education in pain management of the whole staff in the NICU in Bern could explain the differences in pain ratings between the NICUs.

\section{B13}

\section{AN INTERDISCIPLINARY QUALITY IMPROVEMENT APPROACH TO PAIN MANAGEMENT IN THE NEONATAL ICU} B Cofer, B Carter, N Kraft, D Hodge, K Nelson, T Luckett, S Hays, J Deshpande Vanderbilt Children's Hospital, Nashville, Tennessee, USA

BACKGROUND: Pain management in the neonatal intensive care unit (NICU) remains inadequate despite advocacy for improvement.

PURPOSE: To study the impact of an interdisciplinary team approach to improving postoperative pain management in a large tertiary NICU.

METHODS: The study followed the Plan, Do, Study, Act model for improvement (PDSA). We identified a need to improve postoperative pain management in the NICU. We predicted that implementing a plan to educate staff and standardize management would result in better assessment and treatment of pain. A real-time chart audit was conducted to collect data on a convenience sample of 50 postoperative cases in the NICU. The team met weekly to review cases and consider ideas for improving pain management. Following the initial audits of care, the team devised a postoperative pain management guideline utilizing pre-emptive pain management as a continuum from intraoperative anesthesia/analgesia. Recommendations included continuous intravenous fentanyl infusion, "prn" boluses of fentanyl for breakthrough pain, scheduled acetaminophen, and regular pain assessment and reassessment using the N-PASS scale.

RESULTS: Following the initial 50 cases, 2 more sets of 50 charts were reviewed at 6-month intervals as the team continued to target education and standardization. The project has produced a number of clinically useful tools. Improvement has been noted in a number of areas including documentation, use of acetominophen, and a decrease in the use of anxiolytics. Resistance to change has been overcome as improvement has been sustained for 2 years.

CONCLUSIONS: We conclude that a collaborative interdisciplinary team approach results in better postoperative pain management. The consistent use of the PDSA model has yielded desirable and lasting results. This experience is part of an ongoing quality improvement process within the Children's Hospital with generalizability to a number of other clinical settings. * Supported by a grant from the Mayday Fund.

\section{B14}

\section{REPEATED ADMINISTRATION OF SUCROSE REDUCES VENIPUNCTURE PAIN IN NEWBORN INFANTS OF DIABETIC MOTHERS (IDM) AND NORMAL INFANTS}

A Taddio $^{1}$, V Shah ${ }^{2}$, B Stevens ${ }^{1}$, A Ohlsson ${ }^{2}$, J Beyene ${ }^{1}$, J Katz ${ }^{3}$ ${ }^{1}$ The Hospital for Sick Children; ${ }^{2}$ Mount Sinai Hospital;

${ }^{3}$ York University, Toronto, Ontario

BACKGROUND: Sucrose reduces pain in full term neonates when administered as a single dose prior to painful cutaneous procedures. Its effectiveness when used repeatedly, however, has not been documented. Moreover, the effects of sucrose on blood glucose levels are unknown. This 
is particularly important for infants of diabetic mothers (IDM), who undergo multiple painful procedures for monitoring of blood glucose levels. Since all newborns undergo at least two painful procedures, documentation of the sustained effectiveness and safety of repeated administration of sucrose is clinically important.

OBJECTIVE: To determine the analgesic effectiveness and safety of sucrose during the first day of life in infants born to healthy mothers and IDM.

DESIGN/METHODS: Double-blind randomized controlled trial. Healthy neonates within two strata (normal infants and IDM) were randomly assigned to one of two groups (sucrose or water) for all painful procedures in the first day of life. Pain was assessed during venipuncture for the newborn screening test using facial grimacing (brow bulge, eyes squeezed shut, nasolabial furrow). Blood glucose levels were monitored on 3 occasions in the IDM group.

RESULTS: 120 normal infants and 120 IDM were enrolled in the study. The overall mean (SD) gestational age and birthweight was 39 weeks (1 week) and $3.4 \mathrm{~kg}(0.5 \mathrm{~kg})$, with no differences between groups. Repeated measures ANCOVA for facial grimacing revealed a statistically significant main effect of group $(\mathrm{P}<0.001)$, procedure phase $(\mathrm{P}<0.001)$, but not infant strata $(P=0.62)$. All interaction terms were nonsignificant. Overall, the sucrose group showed $65 \%$ less pain than the water group. Blood glucose levels for IDM did not differ between groups ( $\mathrm{P}=0.28$ ). CONCLUSIONS: When used repeatedly during the first day of life, sucrose maintained analgesic effectiveness during venipuncture for the newborn screening test in IDM and normal infants and did not significantly alter IDM blood glucose levels.

\section{B15}

INFANTS AT RISK FOR NEUROLOGICAL IMPAIRMENT: CAN THEY TELL US MORE ABOUT THEIR PAIN THAN CLINICIANS AND PAIN EXPERTS?

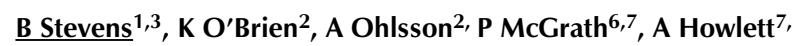
A Dupuis $^{3}$, J Beyene ${ }^{4}$, S Gibbins ${ }^{5}$, L Breau ${ }^{8}$, L Franck ${ }^{9}$

${ }^{1}$ Faculty of Nursing and Medicine, University of Toronto; ${ }^{2}$ Department of Pediatrics, Mount Sinai Hospital, ${ }^{3}$ Population Health Sciences Research Institute; ${ }^{4}$ Statistics in Medicine Unit Research Institute, The Hospital for Sick Children (SickKids); ${ }^{5}$ Interdisciplinary Practice Research and Evidence-Based Practice, Sunnybrook \& Women's College Health Sciences Centre, Toronto, Ontario; ${ }^{6}$ Departments of Psychology and ${ }^{7}$ Pediatrics, Dalhousie University; ${ }^{8}$ Pediatric Pain Service, IWK Health Centre, Halifax, Nova Scotia; ${ }^{9}$ Centre for Nursing and Allied Health Professions Research, Great Ormond Street Hospital for Children, London, United Kingdom

BACKGROUND: Neonates at risk for neurological impairment (NI) are vulnerable to pain and its consequences; however, there have been no validated or clinically useful measures of assessing pain in these infants. OBJECTIVE: To compare physiological and behavioural indicators of procedural pain responses in infants at risk for NI with nurse and expert opinions of indicator importance.

METHODS: A prospective cohort study was undertaken utilizing 3 sets of data: (a) 149 neonates (GA $>25-40$ weeks) at high $(n=54)$, moderate $(n=45)$ and low $(n=50)$ risk for NI from 3 tertiary level NICUs, (b) a representative sample of 95 nurses from the same 3 NICUs (c) 14 international pain experts. Thirteen behavioural and physiological indicators from the infant, nurse and expert data were analyzed.

RESULTS: The 4 variables with the highest accuracy for discriminating 'pain' and 'no pain' across infant NI risk groups were all behavioural: brow bulge $(77-83 \%$ accuracy), eye squeeze $(75-84 \%)$, nasolabial furrow (79-81\%), and total facial expression (78-83\%). Correlations between nurse ratings and infant accuracy scores ranged from moderate to none (mild risk group $\mathrm{r}=0.52, \mathrm{P}=0.07$; moderate $\mathrm{r}=0.43, \mathrm{P}=0.15$; high $\mathrm{r}=-0.12$, $\mathrm{P}=0.69$ ). Experts indicated a better understanding of the importance of the pain variables, with high to moderate correlations between expert ratings and infant accuracy scores (mild risk group, $\mathrm{r}=0.91, \mathrm{P}<0.001$; moderate, $\mathrm{r}=0.85, \mathrm{P}<0.001$; high $\mathrm{r}=0.64, \mathrm{P}=0.019$ ). Both nurses and experts predicted reduced importance of pain indicators in the highest NI risk group.
CONCLUSIONS: Facial actions were the most important variables used to identify pain among infants of all NI risk groups by nurses and experts. Nurses ratings were less associated with these infant pain responses than ratings of experts indicating the need for increased knowledge of pain responses by bedside nurses.

\section{B16}

RESPONSES TO A PAINFUL VERSUS NONPAINFUL PROCEDURE IN INFANTS AT RISK FOR NEUROLOGICAL IMPAIRMENT: PRELIMINARY RESULTS

B Stevens $^{1}$, J Beyene ${ }^{2}$, S Gibbins ${ }^{3}$, A Ohlsson ${ }^{4}$, P McGrath ${ }^{5,6}$, A Howlett $^{6}$, A Finley ${ }^{5,7}$, C Camfield ${ }^{8}$, L Franck ${ }^{9}$, C Johnston ${ }^{10}$ ${ }^{1}$ Faculty of Nursing and Medicine, University of Toronto; Population Health Sciences, Research Institute; ${ }^{2}$ Statistics in Medicine Unit - Research Institute, The Hospital for Sick Children (SickKids), ${ }^{3}$ Interdisciplinary Practice Research and Evidence-Based Practice, Sunnybrook \& Women's College Health Science's Centre, ${ }^{4}$ Department of Pediatrics, Mount Sinai Hospital, Toronto, Ontario; ${ }^{5}$ Departments of Psychology and ${ }^{6}$ Pediatrics, ${ }^{7}$ Departments of Anesthesiology, Dalhousie University; ${ }^{8}$ Departments of Psychology and Pediatrics, Division of Neurology, Pediatric Pain Service, IWK Health Centre, Halifax, Nova Scotia; ${ }^{9}$ Centre for Nursing and Allied Health Professions Research, Great Ormond Street Hospital for Children, London, United Kingdom; ${ }^{10}$ Department of Nursing, McGill University, Montreal, Québec

BACKGROUND: Infants at risk for neurological impairment (NI) have been systematically excluded from research studies; therefore, we do not know if their pain expression differs from infants who are not at risk for NI. OBJECTIVE: To compare infants at high, moderate and low risk for NI pain responses to (a) a diaper change (nonpainful procedure) and (b) a routinely ordered heel lance (painful procedure).

METHODS: Infant physiological (heart rate, oxygen saturation) and behavioural (facial action and cry) pain responses were collected during the pre-established nonpainful and painful procedures. 74 neonates $(\mathrm{GA}>25-40$ weeks; $<2$ weeks PNA) at high $(\mathrm{n}=23)$, moderate $(\mathrm{n}=21)$ and low $(n=30)$ risk for NI were recruited from 3 tertiary level NICUs in Canada and observed during the nonpainful and painful procedures.

RESULTS: RM ANOVA comparing within group (ie, type of procedure) and between group(ie, risk for NI) differences and controlling for procedure order revealed no significant differences between risk groups or type of procedure in the changes from baseline for total facial actions (includes 9 facial actions using the NFCS), and oxygen saturation. Significant changes from baseline occurred after the heel lance versus diaper change for minimum $(F(1,71)=9.16, \quad P=0.003)$, maximum $(F(1,71)=10.99$, $\mathrm{P}=0.001)$ and mean heart rate $(\mathrm{F}(1,71)=10.85, \mathrm{P}=0.001)$. Infants in the moderate risk group demonstrated the highest minimum fundamental frequency for cry $(\mathrm{F}(2,70)=4.06, \mathrm{P}=0.02)$, with no differences found between the diaper and heel lance procedures.

CONCLUSIONS: Risk for NI and type of procedure did not consistently influence infant pain responses. Future research will need to consider factors such as severity of illness and prematurity which may influence the infant's ability to react to procedures that may be both painful and stressful.

\section{B17}

\section{MEASUREMENT OF POSTOPERATIVE AND PROCEDURAL PAIN IN CRITICALLY ILL INFANTS AFTER CARDIAC SURGERY: A COMPARISON STUDY}

$\underline{\text { L Franck }}^{1,2}$, J Peters ${ }^{2}$, D Ridout ${ }^{1}$, R Howard ${ }^{2}$, A Aynsley-Green ${ }^{3}$ ${ }^{1}$ Institute of Child Health; ${ }^{2}$ Great Ormond Street Hospital; ${ }^{3}$ Office of the Children's Commissioner, London, United Kingdom BACKGROUND: Valid and reliable measures for assessing pain in critically ill infants remain elusive and controversy continues as to the utility of behaviour versus physiological indicators. This study compared a range of potential pain indicators in critically ill infants after cardiac surgery at regular intervals during the postoperative period and before and after painful and stressful procedures. 
METHODS: This prospective cohort study used a repeated measures
single sample design. Four observational pain scales (CHIPP, COMFORT, CRIES, and PIPP), cardiorespiratory parameters (HR, HRV, MBP), cortisol, and morphine and its metabolites were obtained for 81 full term newborn infants undergoing major cardiac surgery in the first month of life. Measures were obtained at 6 hourly intervals for the first 48 hours following surgery and before and after stressful (routine nursing caregiving) and painful (chest drain removal) procedures.

RESULTS: Our preliminary analyses have shown good associations between one of the 4 observational pain assessment scales (COMFORT and the cardiorespiratory (HRV), hormonal (cortisol) indices and also with morphine blood levels. The COMFORT scale also showed better discrimination between responses to painful and stressful procedures. We also found moderate associations between observational pain scores and morphine doses administered for chest drain removal. Further multivariate analysis is underway. Random effects modelling is being used to investigate the effects of, for example, analgesia level, time, state (asleep, awake, crying) and procedure in relation to the type of measure. We will also explore the possibility of lag effects.

CONCLUSIONS: Simultaneous measurement of multiple pain and stress indices in the same infants over time provides insight into the responses of critically ill infants during painful and stressful procedures and provides evidence for selecting measures for clinical practice.

\section{B18}

\section{NEURAXIAL ANALGESIA AND RESPIRATORY FUNCTION IN PRETERM INFANTS}

\section{B McClain, M Middleton}

Yale University School of Medicine, New Haven, Connecticut, USA INTRODUCTION: The use of continuous epidural analgesia in neonates is limited, in part, due to fear of respiratory depression. Spinal anesthesia does not avoid CNS or respiratory depression. Studies show that $\mathrm{FiO} 2$ is a reliable indicator of ventilatory function. We investigated the impact of continuous epidural infusions vs spinal anesthesia on the need for ventilatory support in preterm infants.

METHODS: 16 neonates (34.3-2.49 weeks gestation) underwent combined general-regional anesthesia for thoracic or abdominal procedures. 10 received epidural anesthesia which included a loading dose of $0.25 \%$ bupivacaine during surgery. Postoperatively in the epidural group, 5 neonates received fentanyl $1 \mathrm{mcg} / \mathrm{mL}$ in $0.063 \%$ bupivacaine and 5 received hydromorphone $3 \mathrm{mcg} / \mathrm{mL}$ in $0.063 \%$ bupivacaine. All epidural patients received general anesthesia. 6 received single shot intrathecal anesthesia with tetracaine at $0.8 \mathrm{mg} / \mathrm{kg}$ and no supplemental sedation was used.

RESULTS: 5 of 10 infants receiving epidural anesthesia were extubated in the operating room while the remaining epidural patients were extubated within 24 hours. Supplemental oxygen was discontinued within 1 hour of extubation. 8 of the epidural patients were on room air. One patient, gestational age 34 weeks, required ongoing oxygen supplementation at $\mathrm{FiO} 2=0.30$ and was reintubated. 1 of the 6 spinal anesthesia patients required intubation after a high spinal and on chart review, had been extubated only 24 hours prior to surgery. Neonates undergoing spinal anesthesia needed systemic analgesics in the 72 -hour postoperative course.

CONCLUSION:

- The success of avoiding intubation is independent of the type of neuraxial anesthesia.

- A perioperative requirement of $\mathrm{FiO} 2=0.30$ may be a predictor of forthcoming respiratory failure.

- Preterm infants with low indices for respiratory failure may receive continuous epidural analgesia with minimal compromise.
B19

LONG-TERM EFFECTS OF NEONATAL PAIN EXPOSURE ON ACUTE PAIN RESPONSE IN 19 YEAR OLD ADOLESCENTS

T Boelen-Van Der Loo, T Lundqvist

Emma Children's Hospital, Amsterdam Medical Centre, University of Amsterdam, The Netherlands and Uppsala University Children's Hospital, Uppsala, Sweden

OBJECTIVE: To analyze how untreated neonatal pain exposure in preterm neonates influences their acute pain response as 19 year old adolescents.

METHODS: The Cold Pressor Test (CPT) and Visual Analogue Scale (VAS) were used to test Pain THreshold (PTH), Pain TOlerance (PTO) and Pain Intensity (PI) in a group of 19 year olds from the Dutch Project On Preterm and Small for Gestational Age cohort ( $n=567)$. The cohort was divided in two groups, one with an estimated greater neonatal pain exposure $(A n=312)$ and the other with an estimated lesser neonatal pain exposure $(B \mathrm{n}=255)$, and statistically analyzed.

RESULTS: Males $(\mathrm{n}=260)$ demonstrated significantly higher PTH, PTO and lower VAS than females $(n=307)(P<0.005)$. (A)-males $(n=166)$ had higher PTO and lower VAS scores than (B)-males ( $n=94)$ but nonsignificant. Females displayed no such differences (A female $n=146$, B $n=161)$. Small for Gestational Age (SGA) (A)-males $(n=44)$ had significantly higher PTO and lower VAS scores $(\mathrm{P}<0.0034)$ than SGA (B)-males $(n=49)$. AGA females $(n=187)$ had significantly greater PTO than SGA females $(n=120)(P<0.0001)$. AGA males admitted to a hospital more than once had higher PTO than those admitted only once $(\mathrm{P}<0.046)$. In a similar comparison SGA males, AGA and SGA females showed no difference in PTO, PTH or VAS.

CONCLUSION: This study confirms gender difference in acute pain response and pain perception. Females had lower PTH and PTO than their male counterparts. Whilst male PTO increases with the quantity of stress/pain exposure over time, female PTO appears to be unyielding to measurable changes in acute pain response after birth.

\section{B20}

\section{BREASTFEEDING REDUCES PAIN-RELATED STRESS RESPONSES DURING BLOOD COLLECTION FOR THE PKU TEST ON FULL-TERM NEWBORNS}

$\underline{\text { A Moraes Leite }}{ }^{1}$, C Silvan Scochi ${ }^{1}$, M Martins Linhares ${ }^{1}$, J Lander ${ }^{2}$, L Nascimento ${ }^{1}$

${ }^{1}$ University of São Paulo at Ribeirão Preto, College of Nursing, São Paulo, Brazil; ${ }^{2}$ Faculty of Nursing at the University of Alberta, Edmonton, Alberta

The aim of this study was to evaluate the effect of breastfeeding on full term newborns' pain by PKU test. The sample of this randomized clinical trial consisted of 60 full term newborns: one experimental group $(n=31)$, who were placed on the mother's lap and breastfed 5 minutes before and during the entire procedure, and a control group $(n=29)$ who were not breastfed and placed on the mother's lap during the procedure. Ethical approval was obtained and the newborns' parents signed the free and informed consent term before data collection. The heart rate results were compared from the baseline until recovery. The results regarding behaviour parameters (adapted NFCS Scale-brow bulge, eye squeeze, nasolabial furrow) were compared for the three phases: antisepsis, puncture and squeezing; wound compression; and recovery. In experimental group it was also evaluated the suction frequencies. Mann-Whitney's nonparametric test for two independent samples was used to compare both groups, while Friedman's nonparametric test for $\mathrm{K}>2$ dependent samples was used for comparison within the same group, followed by multiple comparisons. Chi-square and Fisher's exact test were used for qualitative variables. ANOVA showed that interaction was significant for the mean values of heart rate. In the experimental group, there was no significant difference between recovery period and baseline related to mean heart rate values. In all analyzed phases, the percentage of facial actions signs was significantly higher for the control group than for the experimental group. As to the variation of mean scores on the NFCS in the same group between different periods, there was a statistically significant difference in both groups. In 
the experimental group, there was a gradual decrease in suction frequency across the procedure. It was concluded that breastfeeding was effective to relieve pain resulting from the PKU test seen all outcome measures.

\section{B21}

\section{MATERNAL SKIN-TO-SKIN CONTACT TO REDUCE PAIN RESPONSE TO BLOOD COLLECTION IN PREMATURES DURING PKU TEST}

T Corrêa Castral ${ }^{1}$, C Silvan Scochi ${ }^{1}$, A Moraes Leite ${ }^{1,2}$

${ }^{1}$ University of São Paulo at Ribeirão Preto, College of Nursing;

2Public Health Nursing, WHO Collaborating Centre for Nursing

Research Development, São Paulo, Brazil

The study aims to test the efficacy of maternal skin-to-skin contact to reduce pain response collection in prematures infants during PKU test. An experimental randomized clinical trial is being carried out at the INUC of a university hospital in Brazil. Preterm newborns $<30$ weeks of gestational age (GA) who are submitted to blood collection for the PKU test are randomly divided in two groups: one experimental, with babies being placed in skin-to-skin care with their mother for 15 minutes before and during the entire procedure, and a control group, with babies kept on their crib or incubator without kangaroo care during the procedure. Ethical approval was obtained and the newborns' parents signed the free and informed consent term before data collection. The collection procedure was divided in seven steps: baseline ( 2 minutes), period after intervention ( 2 minutes), cleaning the heel (20 seconds), squeezing (time enough to blood collection), wound compression (20 seconds) and recovery period ( 2 minutes). Behavioural (facial expression, sleep/wake state, cry) and physiological (heart rate) parameters are recorded to assess the infants' response to pain during the entire collection procedure. NFCS will be scored each two seconds in the first 20 seconds in each procedure phase to capture acute pain response. Sleep/wake state will be analyzed during 30-second blocks each 2 minutes of each phase of the procedure. Cry will be obtained as a percentage of time during the procedure. Heart rate values will be obtained through a cardiac monitor each minute-segment during the entire procedure. We have already conducted a pilot study on August/2005 with 4 newborns, after which we have started data collection. Until now, 56 babies have been filmed ( 31 control $\times 25$ experimental). We rearranged some methodological issues after the pilot study we have conducted.

\section{B22}

\section{EFFECTS OF BREASTFEEDING ON MATERNAL AND NEONATAL BEHAVIOUR DURING PKU TEST}

A Moraes Leite, $C$ Silvan Scochi, M Martins Linhares, T Corrêa Castral, L Monti Fonseca, F Nogueira Góes University of São Paulo at Ribeirão Preto, College of Nursing, São Paulo, Brazil

AIM: The study aimed to compare maternal and newborn behaviour in full term newborns being breastfed during blood collection for the PKU test, in comparison with newborns who were not breastfed.

DESIGN: An experimental randomized clinical trial was carried out at the outpatient clinic of a university hospital. Sixty full term newborns were randomly divided in to two groups: experimental group $(n=31)$, who were placed on the mother's lap and breastfed 5 minutes before and during the entire procedure, and a control group $(n=29)$, who were not breastfed and placed on the mother's lap during the procedure. The behavioural signs were: sleep/awake state, newborn's emotion regulation behaviour, mother's behaviour, and suction were filmed by two cameras. Ethical approval was obtained and the newborns' parents signed the free and informed consent term before data collection started. The results from both groups were compared in each procedural step: (antisepsis/puncture/squeezing), wound compression and recovery (after 5 minutes). Mann-Whitney's nonparametric test for two independent samples was used to compare both groups, while Friedman's nonparametric test for K $>2$ dependent samples was used for comparison within the same group (evolution over time), followed by multiple comparisons.

RESULTS: The control group cried most of the entire collection procedure. In the experimental group the prevailing state was sleep, except during heel puncture. Newborns from the control group showed more signs of emotion regulation, and more mothers showed expressions aimed at comforting the baby. In the breastfeeding group, there was a gradual decrease in suction frequency across the procedure.

CONCLUSION: Both groups showed different behaviour during blood collection for the PKU test. Breastfed newborns and their mothers were calmer than control group.

\section{B23}

ELECTROENCEPHALOGRAPHIC RESPONSE TO PROCEDURAL PAIN IN HEALTHY NEWBORN INFANTS

E Norman ${ }^{1}$, I Rosén ${ }^{2}$, O Ökland ${ }^{3}$, S Vanhatalo ${ }^{4}$, V Fellman ${ }^{1}$, L Hellström-Westas ${ }^{1}$

${ }^{1}$ Department of Paediatrics; ${ }^{2}$ Department of Clinical

Neurophysiology, Lund University Hospital, ${ }^{3}$ Department of Psychology, Lund University, Lund, Sweden; ${ }^{4}$ Department of Clinical Neurophysiology, University Hospital of Helsinki, Helsinki, Finland

BACKGROUND: Recent studies have reported long and delayed ( $>10 \mathrm{~s}$ ) electrical (EEG) or hemodynamic (NIRS - near infrared spectroscopy) responses to painful stimuli in newborns. This study assessed in a larger patient group whether consistent brain responses can be found, and if they could therefore be used as an indicator of painfulness or discomfort.

METHODS: Healthy full term infants $(n=72)$, underwent a nonskinbreaking heel prick $(n=28)$, venous blood sampling $(n=21)$ or capillary blood sampling from the heel $(n=23)$ during monitoring by EEG, video recording and pulse oxymetry. Behavioural responses were scored with Premature Infant Pain Profile (PIPP). EEG reactivity to the painful procedure ( 2 min pre- vs poststimulus) was estimated 1) from discrete $30 \mathrm{sec}$ long time samples with FFT (Fast Fourier Transformation) paradigm, 2) from continuous signal with amplitude integrated EEG (aEEG) paradigm, as well as 3) from continuous time frequency (TF) transformation with wavelet paradigm.

RESULTS: The observed behavioural responses (PIPP) did not show consistent correlations to EEG measures. While there was an overall increase in EEG activity after painful stimulus, a sustained change was statistically significant only at higher $(10-30 \mathrm{~Hz})$ frequencies. There was no consistent laterality in the EEG responses. TF analysis demonstrated, expectedly, significant fluctuation in the EEG activity.

CONCLUSIONS: The present results are consistent with the idea that the delayed, persistent changes in cortical activity may be due to changes in vigilance state, and that EEG activity may not be a useful measure to assess pain in babies.

\section{Treatment Approaches: Interventional, Physical, Psychosocial}

\section{C1}

THE EFFECT OF LOCAL COOLING PRIOR TO VEINPUNCTURE OR INTRAVENOUS CANNULATION ON PAIN-RELATED RESPONSES IN SCHOOL-AGED CHILDREN B Kikhahi ${ }^{1}$, A Movahedi ${ }^{2}$, S Rostami ${ }^{1}$, M Latefi ${ }^{1}$

1Ahvaz Jondishapour University of Medical Sciences;

2Semnan University of Medical Sciences, Semnan, Ahvaz, Iran

The purpose of this study was to determine the effect of local cooling prior to veinpuncture or intravenous cannulation on pain-related responses in school-aged children. The subjects were 80 children aged 6-12 years, who were selected on referral to the pediatric emergency department of Ahwaz University of Medical Sciences hospitals. For this purpose (effectiveness of local cooling) two groups of patients participated; in the test group, prior to veinpuncture or intravenous cannulation, the site of injection was cooled for 5 minutes with a icebag. While, in the control group, the procedure was performed by usual routine method. Physiological responses (blood pressure, pulse and respiration), behavioural responses (crying, facial expression, child verbal, torso, touch and leg movement) and 
subjective responses (intensity of pain), were measured in two groups. The physiological responses were measured before and 5 minutes after procedure was completed. The behavioural responses were measured during and 5 minutes after the procedure and subjective responses were measured 5 minutes after the procedure.

The results of this research showed no significant differences between physiological responses (before and after procedure) between the two groups $(\mathrm{P}>0.1)$. but, behavioural responses during and after the procedure $(\mathrm{P} 0.005)$ and subjective responses after the procedure $(\mathrm{P}<0.05)$ were lesser in the test group than control group.

These results suggest that the use of local cooling prior to veinpuncture or intravenous cannulation to be a simple and effective intervention in reducing the procedural-related pain.

\section{C2}

\section{COMPARISON OF EMLA CREAM EFFECTIVENESS AND LOCAL APPLICATION OF COOLING ON VENIPUNCTURE PAIN IN SCHOOL AGE CHILDREN 2005}

S Rostami, Z Sabzi, H Alijany, A Rasekh

Ahwaz Jondi Shapour University of Medical Sciences, Ahwaz, Iran Veinpuncture is commonly seen as one of the most painful and frequent invasive procedures performed by nurses. As advocates for children, nurses are obligated to minimize the emotional and physical effects of painful procedures and they have to use simple, available, and rapid method for decreasing of pain. Different studies related to local anesthesia have shown that pain decrease is important in children.

AIM: This research is a clinical trial study. The purpose of this study was to compare EMIA cream effectiveness and local application of cooling on veinpuncture pain in school age children. The subjects were children aged 7-11 primary school girls. They were selected purposively for health and anemia assessment, during referrals to Sijaval Medical Health Center in the city of Bandar Turkmen.

METHOD: In this randomized study 75 girls 7-11 years old were divided in to three equal groups (control, local cool, EMLA). In the EMLA group 45 minutes before veinpuncture, 1.5 gr EMLA cream was used on the puncture site with Tegaderm dressing. In the local cool group 3 minutes before procedure, the puncture site was cooled with an ice bag enveloped in a cloth, and in the control group EMLA was not used and local cool. Then 5 minutes after blood sampling, pain intensity was measured by numeric Oucher scale in three groups.

FINDINGS: In this study, $48 \%$ of control group children reported middle pain during veinpuncture. Mean Oucher scale were significantly lower in EMLA group $(M=21.20)$, and in the local cool group $(M=19.60)$ than control group (56.40). Statistically there was not significant difference in pain intensity between cool $(\mathrm{P}>0.05)$. There were significant differences in pain intensity between EMLA and control group $(\mathrm{P}<0.05)$, and between control and local cool groups.

CONCLUSION: The results of this study showed that both EMLA and local cool decreased pain intensity of veinpuncture in school aged children. Regarding the equal effects of EMLA and local cool, the use of local cool (ice) before veinpuncture is recommended. The reason is that it is simple, safe, easy to use, inexpensive and an effective intervention for reduction of veinpuncture pain in children.

\section{C3}

\section{A SELF-MANAGEMENT APPROACH TO PROCEDURAL PAIN AND DISTRESS: THE COMFORT KIT FOR KIDS AND FAMILIES}

\section{T Culbert, M Fitzgerald, M Finkelstein, P Whitehead, K Dixon} Children's Hospitals and Clinics of Minnesota, Minneapolis, Minnesota, USA

BACKGROUND: The management of pain in pediatric settings is an important issue. Good pain management requires a multimodality approach with the use of analgesics along with cognitive and behavioural techniques. Scientific studies support the efficacy of education, distraction and relaxation in procedural pain management with children. Parents are interested in helping children manage pain but often feel inadequate in
D

the face of surgical, procedural, or chronic pain. The proven benefits of self-management of pain include mastery and confidence leading to less anxiety, fostering an internal locus of control, development of life skills that can be applied to future circumstances. Self-management techniques are often fun and engaging for children resulting in active participation and cooperation in care and decision making and decreasing staff time and need for medication.

OBJECTIVES: The objectives of this study were to develop and test a "Comfort Kit" for children over the age of 7. The goal of creating the "Comfort Kit" was to 1) Incorporate current knowledge of cognitive and behavioural interventions into a format that would be appealing to children over the age of 7.2) Emphasize self-management techniques in the components of the kit. 3) Develop parent coaching materials. 4) Package it all in a novel, graphically rich, and cost-effective form. 5) Utilize follow-up surveys of users to determine satisfaction with the kits and materials. 6) If successful, consider ways in which to share this approach with other health care organizations that serve children

DESIGN/METHODS: Comfort Kits were designed by Dr Culbert with input from Youth Advisory Committee, Child Life, and the Pain Team and were reviewed for safety by risk management. The kits included ply/distraction items - bubbles, massage pen, stress card, pinwheel, essential oil blend, stickers, Visual analogue scale ruler, and a stress ball. Three booklets were developed with editorial assistance from a patient education specialist: Getting Started, Being a Good Comfort Coach, and How to Help Yourself. Kits were mailed to children who were scheduled for day surgery and were given to families in the Diabetes and Hematology/Oncology clinics. Follow-up telephone surveys were done. RESULTS: Initially, approximately 200 kits were distributed through various departments with families being asked to voluntarily participate in the study. Requirements were only that enrollees had to be age 7-19, have a likelihood of experiencing procedural pain or distress in the upcoming $2-3$ weeks and be willing to answer questions via a brief telephone survey 2-3 weeks after receiving the kit. Of the 97 parents who responded, $59 \%$ were female. Mean age of children using the kits was 10.8 years with a range of $7-19$ years.

Over $90 \%$ of the parents and nearly $80 \%$ of the children surveyed found the kit to be somewhat or very helpful. Over $90 \%$ of parents and kids would recommend the Comfort Kit to others. Over 92\% of the Parents and over $83 \%$ of the kids found the booklets easy to understand. The most commonly utilized items in the kits were the massage pen $(80.5 \%$ of respondent parents and $91.7 \%$ of kids), squeeze ball (79.3\% of parents and $87.5 \%$ of kids) and the stress biofeedback card $(69.5 \%$ of parents and $87.5 \%$ of kids). In terms of techniques tried, $39 \%$ of parents reported using the relaxation breathing with their child before or after a medical procedure, $34 \%$ reported using the mental imagery exercise, and 33\% reported using the muscle relaxation exercise. In terms of the written materials in the booklets, $37.5 \%$ of the parents surveyed stated that the section with general information on pain and comfort for kids and $37.5 \%$ also stated that the basic descriptive information about the items in the Comfort Kit and how to use them was useful.

CONCLUSIONS: The novel, graphically rich, self-management oriented "Comfort Kit" vehicle provides a low cost, time-efficient method to improve self-management skills for children undergoing painful procedures and reduce the amount of experienced pain and distress. Further research is needed to determine broader applicability, effects on procedural cooperation and whether this intervention is reasonable for other pediatric populations.

\section{C4}

\section{THE EFFECTS OF A TWO-WEEK TRIAL OF TRANSCUTANEOUS ELECTRICAL NERVE STIMULATION FOR PEDIATRIC CHRONIC BACK PAIN}

$\underline{\text { S Van Epps }}{ }^{1}$, W Zempsky ${ }^{1}$, N Schechter ${ }^{1}$, L Pescatello ${ }^{2}$, T Lerer ${ }^{1}$ ${ }^{1}$ Connecticut Children's Medical Center, Hartford; ${ }^{2}$ University of Connecticut, Storrs, Connecticut, USA

PURPOSE: The purpose of this study was to determine the effectiveness of a 2-week trial of high frequency Transcutaneous Electrical Nerve Stimulation (TENS) added to the subjects' conventional treatment for pediatric chronic back pain. 
METHODS: The study was originally designed as a quasi-experimental crossover research design with a one-week washout period. Subjects were randomly assigned to one of two groups to determine the order of receiving the TENS treatment. Due to poor subject recruitment the study was concluded with five subjects, one who received the TENS plus conventional treatment in the first 2-week treatment and four in the second 2-week treatment period. We have elected to report the results of the subjects', who received the TENS plus conventional treatment during the second treatment period. The subjects $(n=4)$ were females average age $15.5(\mathrm{SD}=0.6)$. The TENS treatment consisted of high frequency TENS performed for 1 hour, 3 times a day for 2-weeks at home. Pain intensity (Visual Analogue Scale), activity level (Pedometer readings), and healthrelated quality of life (Pediatric Quality of Life Survey - PedsQL) mean scores were compared after the 2 -weeks of TENS treatment combined with their conventional treatment and after 2 -weeks of their conventional treatment alone with a two-tailed, paired $t$-test with á $<0.05$ established as the level of statistical significance.

RESULTS: The subjects' mean PedsQL Total score increased $(\mathrm{P}=0.002)$ after 2-weeks of TENS combined with conventional treatments compared to 2-weeks of their conventional treatments alone. There was no significant change in the subjects' mean pain intensity and activity level scores. CONCLUSION: This study suggests that TENS may be an effective tool in treating children who have chronic back pain as well as providing recommendations for future research.

\section{C5}

\section{ACUPUNCTURE FOR THE MANAGEMENT OF PAIN AND EMERGENCE AGITATION AFTER BILATERAL MYRINGOTOMY TUBE INSERTION \\ $\underline{Y ~ L i n}^{1}$, R Tassone ${ }^{2}$, S Jahng ${ }^{2}$, D Zurakowski ${ }^{2}$ \\ 1Children's Hospital Boston; ${ }^{2}$ Harvard Medical School, Boston, Massachusetts, USA}

INTRODUCTION: Bilateral myringotomy tube placement is the second most frequently performed pediatric surgical procedure, following circumcision (1). The procedure is brief. Sevoflurane is a common anesthetic agent for this procedure. The safety and efficacy of sevoflurane has been well documented. It has also been associated with an increased incidence of postoperative agitation upon emergence from anesthesia. This study is to evaluate the use of acupuncture for the management of emergence agitation and pain after bilateral myringotomy tube insertion in pediatric patients.

METHODS: This prospective, patient and assessor-blind, randomized controlled trial was approved by the Institutional Review Board. Pediatric patients aged between 1 and 6 years, ASA physical status I or II, and without previous history of myringotomy tube surgery were recruited for the study. After obtaining parental consent, the patients were randomized into one of the two groups using a computer-generated random number table. All the patients received a standardized general anesthesia technique with the parents present during induction. The anesthetic agents included oxygen, nitrous oxide, and sevoflurane. Spontaneous ventilation was maintained and anesthetic depth was adjusted to meet each individual's clinical needs. Once the patient was adequately anesthetized, one group of patients received an acupuncture treatment prior to the surgical procedure. The other group of patients received the standard anesthetic and had no acupuncture treatment. Acupuncture at LI-4 (ho gu) acupuncture point and HT-7 (shen men) acupuncture point was used for this study. Manual manipulations without electrical stimulation were utilized for acupuncture treatment. At the time of surgery, the surgeon would report the condition of the middle ear. Anesthetic emergence was evaluated in the Post Anesthesia Care Unit (PACU). An observer, who did not know the patient's group allocation, assessed each patient's recovery from anesthesia in the PACU using agitation score and CHEOP pain score every five minutes until the patient was discharged from the hospital.

RESULTS: Sixty patients, 38 male and 22 female with mean age $2.2 \pm 1.4$ years $($ mean $\pm \mathrm{SD}$ ) were enrolled in this study. There was no significant difference between those patients who received acupuncture treatment as compared to those who did not receive acupuncture with respect to the gender, age, weight, surgical and anesthesia time, and the condition of middle ear disease. Repeated measures of the analysis of variance (ANOVA) indicated a statistically significant reduction in postoperative pain score at all time intervals in those patients who received acupuncture treatment, however the rate of change of pain over time was similar between the two groups. There was a statistically significant reduction in postoperative agitation in the patients who received acupuncture when compared to those who did not, and the rate of decline of postoperative agitation was faster among those patients who received acupuncture treatment.

Although there was no difference in the duration of PACU stay, the need for postoperative analgesia (acetaminophen) was significantly reduced in those patients who received acupuncture (57\%) compared to those who did not $(87 \%)$.

DISCUSSION: Our study indicates that acupuncture may be a useful therapy for the treatment of emergence agitation and pain management after myringotomy tube insertion in pediatric patients. Acupuncture significantly reduces both postoperative pain and emergence agitation, and facilitates the rate of recovery from emergence agitation. Further research should be done to evaluate the effects of acupuncture at various stages of general anesthesia.

\section{REFERENCES}

1. Boston M, McCook J, Burke B, Derkay C. Incidence of and risk factors for additional tympanostomy tube insertion in children. Arch Otolaryngol Head Neck Surg 2003;129:293-6.

\section{C6 \\ PARENTAL COACHING DURING CHILDREN'S VENIPUNCTURES \\ $\underline{\text { B Finnström }}^{1,2,3}$, B Käck1,2 \\ 1The Queen Silvia Children's Hospital; ${ }^{2}$ Sahlgrenska University Hospital, Göteborg, Sweden}

A child's pain experience is complex and the pain may increase because of the child's stress and fear (1). Several authors have pointed out the significance of parental support to children undergoing medical procedures, and research has shown that active participation can decrease the parents' stress and anxiety $(2,3)$.

AIMS AND METHOD: The aims of the study were to evaluate the influence of structured advice and instructions concerning the parent's actions and support during venipuncture on

- the parent's stress/anxiety

- the child's experience of pain and stress/anxiety.

40 parents and children (aged 5-12) participated in the study. All children in the study group were remitted to the ward because of a medical investigation, which included venipuncture. 20 children were randomized to the intervention group and 20 to the control group. Children, who had ongoing pain, did not speak Swedish or had developmental delays, were excluded. The parents in the intervention group were carefully informed about how to act during the venipuncture and were instructed to participate actively in the child's distraction.

The child scored its pain and stress/anxiety before and during the procedure. The parent scored his/her and the child's stress/anxiety before and during the procedure. The pain was reported with Coloured Analogue Scale (CAS) and the stress/anxiety with Facial Affective Scale (FAS). The parent also scored his/her estimated ability to support the child during the procedure on an ordinal scale with end points minimum and maximum. The assisting nurse scored the child's pain and stress/anxiety and the parent's ability to support the child on a similar ordinal scale. RESULTS: Preliminary results will be presented on the conference.

\section{REFERENCES}

1. McGrath PA (1990). Pain in Children. Nature, Assessment \& Treatment. New York; The Guilford Press, 1990.

2. Blount RT, Bachanas PJ, Powers SW, et al. Training Children to Cope and Parents to Coach Them During Routine Immunizations: Effect on Child, Parent, and Staff Behaviors. Behav Ther 1992;23:689-705.

3. Cavender K, Goff MD, Hollon EC, Guzzetta CE. Parents' 
C7

\section{CLINICAL EXPERIENCE OF NASAL SUFENTANIL AND S-KETAMINE FOR THE TREATMENT OF PROCEDURAL PAIN IN CHILDREN WITH BURN INJURIES}

$\underline{\text { S Lundeberg}^{1} \text {, A Lindberg }}{ }^{2}, M$ Segerberg ${ }^{2}$

${ }^{1}$ Pain Treatment Service, Lidingoe; ${ }^{2}$ Pain Treatment Service, Stockholm, Astrid Lindgren Children's Hospital, Karolinska Institute, Sweden

BACKGROUND: Procedural pain is a common problem when caring for the child with burn injuries. There is a need for short-acting and safe drugs without postprocedural sedation. An alternative to nitrous oxide, when lacking an iv route, is using nasal sufentanil with or without s-ketamine. Sufentanil is a fast and relatively short acting potent opioid. S-ketamine (NMDA-antagonist), the left enantiomer of ketamine, has both an analgesic and sedative effect.

METHOD: Children are given oral medication with paracetamol $40 \mathrm{mg} / \mathrm{kg}$ and diklofenac $1 \mathrm{mg} / \mathrm{kg}$ or ibuprofen $10 \mathrm{mg} / \mathrm{kg}$ two hours previous to the procedure. Sufentanil $0.5-1 \mathrm{microg} / \mathrm{kg}$ is administered by the nasal route. The lower dose is used when combined with s-ketamine $0.5 \mathrm{mg} / \mathrm{kg}$. The sedative effect is evident after 10 to 15 minutes and maximal analgesic effect is achieved after 20 to 25 minutes.

When sufficient analgesia is achieved the burn dressing is removed and gauzes with buffered lidocaine $(1.5 \mathrm{~mL}$ of sodium bicarbonate $0.6-1$ molar to $10 \mathrm{~mL}$ of lidocaine) are placed on the burned area for 10-15 minutes before revision of the burned area. The described method is used in procedures include dressing changes, bathing and physiotherapy.

The effect of the nasal drugs weans off in roughly one hour. Skilled personnel in taking care of a child's airway (ie, anesthesia or emergency staff) must be present due to the diminutive risk of respiratory depression.

RESULTS: A majority of the children (about 150 treatments 2005) accepted the procedure well with good pain relief. Nausea/vomiting were the most common side effect but only occurred occasionally. In some children nasal s-ketamine gives a "stinging" feeling in the pharynx which lasts for a couple of minutes. An alternative is to use the oral instead of the nasal route when using s-ketamine.

CONCLUSION: Our clinical impression is that nasal administration with sufentanil $\pm \mathrm{s}$-ketamine is an attractive alternative for the treatment of procedural pain in the burned child who lacks an iv catheter.

\section{C8}

VR ANESTHESIA: PEDIATRIC PAIN DISTRACTION FOR IV PLACEMENT

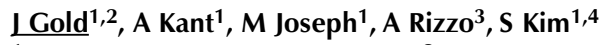

${ }^{1}$ Childrens Hospital Los Angeles; ${ }^{2}$ USC Keck School of Medicine, Los Angeles, ${ }^{3}$ USC Institute for Creative Technologies, Marina del Rey, California, USA; ${ }^{3}$ Hanyang University College of Medicine, Seoul, Korea

Routine invasive medical procedures, such as venipuncture, continue to cause distress in pediatric medical settings. In the past two decades, pediatric pain experts have identified immersive technologies (eg, Game Boy, virtual reality) as preferable to less immersive forms of distraction (eg, cartoons, toys). The aim of this study was to test the efficacy of virtual reality (VR) as a pain distraction for pediatric IV placement. Twenty children (12 boys, 8 girls) requiring IV placement for an MRI/CT scan were randomly assigned to the following conditions: 1) VR distraction (Street Luge, 5DT) presented via a head-mounted display or 2) standard of care (topical anesthetic) with no distraction. Participants completed self-report questionnaires measuring several health-related outcomes prior to and following the procedure. Children in both conditions reported preprocedural anxiety sensitivity (vulnerability to stressful situations; VR: $M=29.6$; Control: $M=29.0$ ) comparable to that of a pediatric clinical sample of children with diagnosed anxiety disorders $(M=28.1$; Silverman, Fleisig, Rabian, \& Peterson, 1991). Results indicated that children in the control condition reported a fourfold increase in affective pain $\left(t=-3.25^{*}\right)$ on the
DPA

Faces Pain Scale - Revised, unlike participants in the VR condition $(t=-1.00)$. None of the participants assigned to the VR treatment condition cried or required physical restraint during the procedure; by contrast, one participant in the control condition cried throughout the procedure, requiring physical restraint. VR pain distraction (Street Luge) during IV placement was associated with an adequate level of presence, no simulator sickness, and increased pain management satisfaction. VR is a promising tool for pediatric IV placement as it provides an immediate and effective distraction from the associated distress of invasive medical procedures. Further investigations should target larger sample sizes and matching participants with procedures and interventions.

\section{C9}

\section{NURSES AND DOCTORS UNDERSTANDING OF USING DISTRACTION WITH CHILDREN}

\section{Griffin}

Capital \& Coast District Health Board, Wellington, New Zealand

One of the key aims of health professionals is to minimize the distress and uneasiness that children feel during procedures and investigations. A key to doing this is the use of distraction. Despite a large body of research and literature on the usefulness of distraction and the positive impact it has for children there is little evidence as to when, why, and what is used by staff in busy wards and departments. There is very little research or evidence that informs what happens in the natural setting of a clinical workplace. The majority of research and evidence on its usefulness has been carried out in research settings, with time, equipment and instruction available to staff. The aim of this research was to find out what are nurses' and doctors' understandings and experiences of using distraction with children during procedures. A postal survey of 110 nurses and doctors across a general Emergency Department and inpatient children's wards of a large tertiary teaching hospital was undertaken. Forty-five $(41 \%)$ people responded to the survey consisting of 36 nurses and 9 doctors.

The findings suggest that there is not only, at times, a lack of knowledge and experience in the use of distraction, but also a lack of understanding by some about what it is, when to use it, and for whom it works. Findings indicate that an individual's profession, past experience or length of time working with children has no impact on their use of distraction. Some staff felt that distraction is of benefit to all children and use it all the time, while others feel it is ineffective in certain age groups, particularly in the under one year olds and the 11-16 year old age groups. A variety of distraction techniques were used by the staff which included, bubbles, verbal interactions and music.

Results from the survey yielded some interesting assumptions by staff and points for discussion. However along with answering questions on distraction, questions were raised which would be worthy of investigation in the future. The main benefit of this research has been to identify a deficiency in the understanding of distraction and this has opened up possibilities for education that can now be implemented.

\section{C11}

\section{TEACHING PARENTS TO PROVIDE DISTRACTION COACHING FOR THEIR CHILDREN DURING MEDICAL PROCEDURES}

\section{$\underline{\text { L Myers, }}$ C Kleiber, A McCarthy, K Hanrahan}

University of lowa, lowa City, lowa, USA

PROBLEM: While most parents want to be with their children during medical procedures, many state they do not know how to help their children cope. Distraction is an effective intervention for helping children cope with medical procedures. Training may increase the frequency and quality of parent distraction coaching. A brief, effective teaching package is needed to train parents. The purpose of this study is to test an intervention designed to teach parents to use distraction with their children during medical procedures.

THEORETICAL FRAMEWORK: The teaching intervention was guided by Bandura's self-efficacy theory using videotaped parent models to demonstrate the use of effective distraction. 


\section{METHODOLOGY:}

Design: Randomized controlled trial

Subjects: 540 parents present with their children during scheduled intravenous (IV) catheter insertions.

Instrument: The Distraction Coaching Index (DCI) is a behaviour observation instrument for measuring the frequency and quality of distraction. Construct validity and inter-rater reliability of this tool have been established.

Procedures: Parents randomized to the distraction intervention teaching package watched a seven-minute video, received written instructions, and discussed a distraction plan with the RA. All subjects had distraction materials available for use during the IV insertion. Parents were videotaped during their child's IV insertion.

Analysis Method: Videotapes of parent behaviour will be scored using the DCI. The DCI scores for the experimental and control groups will be compared using the Wilcoxon Rank Sum test.

RESULTS: Data collection will be completed by February 2006 and preliminary analyses will be reported.

IMPLICATIONS: If a brief teaching intervention is shown to be effective in teaching parents to use high quality and frequent distraction during medical procedures, it could be a useful and practical tool in the clinical setting that may benefit both parents and children.

\section{C12}

\section{PARENT SATISFACTION WITH SPASTICITY TREATMENT IN CEREBRAL PALSY}

\section{F Symons $^{1}$, P Rivard ${ }^{2}$, A Nugent ${ }^{2}$}

'University of Minnesota, Minneapolis; ${ }^{2}$ Gillette Children's

Specialty Healthcare, St Paul, Minnesota, USA

OBJECTIVES: To evaluate parent satisfaction associated with Botulinum toxin type A (BtxA) injection for spasticity in cerebral palsy.

DESIGN: A single-point cross sectional survey design with standardized measures of parent satisfaction (Treatment Evaluation Inventory; TEI) specific to BtxA injection.

SETTING: Regional specialty health care centre medical clinic and pain research program.

PARTICIPANTS: Fifty-nine parents of children with cerebral palsy receiving BtxA injection for spasticity.

RESULTS: Overall parent rating of treatment acceptability ranged from moderate to high. TEI item-total correlations were high $(0.7, \mathrm{P}<0.001)$. There were no significant differences for caregiver ratings in relation to rater (sex, marital status, income) or child (sex, cognitive status, severity of disability) characteristics.

CONCLUSIONS: These findings indicate that parents of children with cerebral palsy consider BtxA treatment for the management of spasticity to be an acceptable form of treatment despite observations that for some children the procedure is associated with pain. Overall, these results suggest that BtxA is a socially valid treatment from a parent perspective for the management of spasticity in children with developmental disability associated with cerebral palsy.

\section{C13}

\section{PAIN, CEREBRAL PALSY, AND BOTULINUM TOXIN INJECTION}

P Rivard $^{1}$, A Nugent ${ }^{1}$, F Symons ${ }^{2}$

${ }^{1}$ Gillette Children's Specialty Healthcare, St Paul; ${ }^{2}$ University of

\section{Minnesota, Minneapolis, Minnesota, USA}

OBJECTIVES: To evaluate parent reported pain before and after Botulinum toxin type A (BtxA) injection for spasticity treatment in children with cerebral palsy.

DESIGN: Prospective with pre- and postbotox injection measures of pain frequency/intensity and pain interference using the Dalhousie Pain Survey (DPS) and the Brief Pain Inventory (BPI).

SETTING: Regional specialty health care centre medical clinic and pain research program.

PARTICIPANTS: Thirty-four parents of children with cerebral palsy receiving BtxA injection for spasticity.
DPPC, DO NOT COPY

RESULTS: Overall parent rating of pain was significantly reduced following BtxA injection (Proportion=0.382; 95\% CI of 0.234-0.546). There were no significant differences for males or females.

CONCLUSIONS: Although there is considerable evidence that BtxA is highly efficacious for the treatment of spasticity associated with cerebral palsy; there is little direct evidence specific to associated features (eg, pain). This is the first study to directly ask parents about their child's pain pre- and postBtxA treatment. Overall, the results suggest that BtxA treatment for spasticity results in significant pain reduction.

\section{C14}

COMPARISON OF NALOXONE AND SALINE INFUSIONS FOR TREATMENT OF OPIOID-INDUCED PRURITUS IN CHILDREN WITH CONTINUOUS EPIDURAL INFUSION: A PROSPECTIVE, RANDOMIZED DOUBLE-BLINDED, PLACEBO-CONTROLLED TRIAL

C Frigon, E Hunter, $M$ Ranger, J Desparmet

Montreal Children's Hospital/McGill University Health Center, Montréal, Québec

INTRODUCTION: Pruritus is the most frequent side effect resulting from epidural opioids and may lead to significant discomfort, or even discontinuation of epidural infusion. We proposed to determine if prophylactic administration of intravenous naloxone infusion decreases the incidence and severity of opioid-induced pruritus in children with continuous epidural therapy for postoperative analgesia.

METHODS: We designed a prospective, randomized, double blind trial. We are currently recruiting children aged between 4 and 12 years old scheduled for elective surgery, in which postoperative epidural analgesia is chosen. Children with epidural analgesia are randomized to one of the following group: children in group 1 receive an intravenous naloxone infusion at $1 \mathrm{mcg} / \mathrm{kg} / \mathrm{hr}$; children in group 2 receive an infusion of saline at $0.1 \mathrm{~mL} / \mathrm{kg} / \mathrm{hr}$. Postoperatively, an observer blinded to the intravenous solution assesses pruritus, nausea and vomiting, pain, sedation, and vital signs every four hours in a standardize fashion for 48 hours or until the discontinuation of the epidural infusion.

PRELIMINARY DEMOGRAPHIC RESULTS: We have recruited over 20 patients of the sample size of 56 . Children have a mean age of 7.9 years $( \pm 3.9)$, mean weight of $26.5 \mathrm{~kg}( \pm 8.8)$ and are classified as ASA 1,2 , or 3 . The majority of scheduled surgeries were germane to urology.

CONCLUSIONS: The completion of the study should allow us to determine if intravenous infusion of naloxone is effective to prevent postoperative opioid-induced pruritus in children with continuous epidural infusions.

\section{C15}

\section{OPIOID-RELATED SIDE EFFECTS ASSOCIATED WITH SCHEDULED ORAL ANALGESIC DOSING IN PRESCHOOL CHILDREN FOLLOWING TONSILLECTOMY}

K Sutters ${ }^{1,2}$, C Miaskowski ${ }^{2}$, S Paul ${ }^{2}$, S Waite ${ }^{1}$, M Savedra ${ }^{2}$, D Holdridge-Zeuner ${ }^{2}$, K Mahoney ${ }^{2}$, B Lanier ${ }^{3}$

${ }^{1}$ Children's Hospital Central California, Madera; ${ }^{2}$ University of California, San Francisco; ${ }^{3}$ Central California Ear, Nose \& Throat Medical Group, Fresno, California, USA

As part of a study that evaluated the feasibility of scheduled dosing of analgesics with preschool children (ages 3 to 5 years; $N=47$ ), in their home following tonsillectomy, the purpose of this study was to determine the frequency of moderate-to-severe opioid-related side effects. Parents were taught to administer acetaminophen with hydrocodone every 4 hours around-the-clock for the first 3 days following surgery. Parents recorded on a daily basis if their child experienced any of the following side effects: daytime sedation, lightheadedness or feeling dizzy, nightmares, nausea, vomiting, and constipation. If the side effect occurred, its severity was rated on a 4-point Likert scale (ie, slight, moderate, severe, or very severe). Side effects were recoded into a dichotomous response (ie, $0=$ did not have or slight symptoms or 1 = symptoms that were moderate, severe, or very severe) for statistical analyses. Significant decreases in the frequency of moderate to severe daytime sedation, nausea, and vomiting (all $\mathrm{P}<0.04$ ) 
were found over the four postoperative assessments. Significant increases in the frequency of moderate-to-severe constipation were found over the four postoperative assessments $(\mathrm{P}=0.005)$. Findings suggest that preschool children receiving scheduled dosing of acetaminophen with hydrocodone experience moderate-to-severe opioid-related side effects.

Supported by a grant from NINR-NR04826.

\section{C16}

\section{IS THE SHOTBLOCKER EFFECTIVE IN REDUCING IMMUNIZATION PAIN?}

$\underline{\text { R Foster }}^{1,2}$, T Eberhart ${ }^{2}$, J Zuk ${ }^{1,2}$, C Finn ${ }^{1,2}$

'University of Colorado at Denver; ${ }^{2}$ The Children's Hospital,

Denver, Colorado, USA

AIMS: To test whether the Shotblocker $囚$, in combination with usual comfort measures, is effective in reducing immunization pain.

BACKGROUND: The Shotblocker ${ }^{\circledR}$, a small, flexible plastic, noninvasive device with blunt skin contact points on the underside, is designed to reduce needle-related pain by putting pressure on large diameter (A beta) fibres. Little research exists, however, to support the manufacturer's claim that the device "instantly blocks" injection pain.

METHODS: In a two-group, randomized, controlled study, subjects were recruited at a large immunization fair and randomized to the experimental or control group. All children received usual comfort treatments guided by Child Life staff. For children in the experimental group, a Shotblocker $\AA$ was used for each immunization. Following immunizations, children and their parents answered a short survey and rated immunization pain using the Bieri Faces Pain Scale.

RESULTS: A sample of 171 children, ages 3 months through 17 years $(\mathrm{M}=6.68, \mathrm{SD}=4.1)$ participated from 120 families. Children had from 1-7 immunizations $(\mathrm{M}=2.96, \mathrm{SD}=1.5)$, with younger children receiving more immunizations than school age children or adolescents $(r=-0.227$, $\mathrm{P}<0.01$ ). Parents held their children for immunizations in $63 \%$ of the cases and $73 \%$ of the children used alternative focus modalities such as bubbles or a pinwheel. Only 23 children received neither supportive positioning nor alternative focus modalities. The Shotblocker ${ }^{\circledR}$ did not distinguish between immunization pain for the experimental and control groups $(\mathrm{F}=0.0006, \mathrm{P}=0.94)$. Pain varied only by supportive positioning and age. Children who were held supportively by a parent had significantly more pain $(t=6.71, P<0.001)$. They may have been held because they were younger and received more injections. In this study, the Shotblocker $\AA$, when combined with other comfort measures, was ineffective in reducing immunization pain.

\section{C17}

EXPOSURE AND ACCEPTANCE STRATEGIES VS A MULTIDISCIPLINARY APPROACH INCLUDING AMITRIPTYLINE FOR CHILDREN AND ADOLESCENTS WITH CHRONIC DEBILITATING PAIN - A RANDOMIZED CONTROLLED TRIAL

R Wicksell ${ }^{1}$, J Ahlqvist ${ }^{1}$, L Melin ${ }^{2}$, G Olsson ${ }^{1}$

1Pain Treament Service, Astrid Lindgren Children's Hospital, Karolinska University Hospital; ${ }^{2}$ Department of Psychology, Uppsala University, Stockholm, Sweden

AIM OF INVESTIGATION: For many patients with disabilities due to chronic pain of unclear origin, a multidisciplinary treatment is commonly considered the treatment of choice. Recently, an approach that promotes acceptance of pain is suggested. In a randomized control trial, we compared an exposure-based behaviour therapy emphasizing acceptance to a multidisciplinary approach including amitriptyline for children and adolescents with chronic idiopathic pain.

METHODS: 32 patients (10 to 18 years) referred to the clinic because of long-standing debilitating pain were randomized to one of the two treatment conditions.

In the experiment group, exposure was considered the core intervention. Acceptance strategies were used to facilitate the exposure process. Rehabilitation consisted of approximately 10 weekly sessions with the patient and 2 with the parents. In the control group, amitriptyline was
DPC, DO NOT COPY

administered within a multidisciplinary approach during a period of 3 months.

Assessments were performed pre- and post-treatment and at 3 and 6 months follow-up. Measurements included functional disability, pain (intensity and interference), pain-related discomfort, school absence, pain medications, and coping strategies.

RESULTS: Preliminary results $(n=22)$ are analyzed. Improvements in functional disability and pain were seen for both groups. However, a disability index (impact of pain on daily living), and a discomfort index (pain-related worrying), showed a significant interaction effect in favour of the exposure intervention.

CONCLUSION: The results indicate the effectiveness of an exposurebased behaviour therapy emphasizing acceptance in increasing the level of functioning, and decreasing the level of pain-related discomfort, in children and adolescents with chronic pain.

\section{C18}

\section{EXTENDED FOLLOW-UP IN POSTOPERATIVE PAIN}

E Villeneuve, S Charette, L Cyrenne, $M$ Haig

Université de Montréal, Montréal, Québec

INTRODUCTION: Patients who undergo major surgical procedures often benefit from intensive, integrated pain management by a dedicated team in the first few days following their intervention. However, at the end of the PCA or epidural approach, the patient usually returns to the sole care of his surgeon and the transition from pain team to surgical team often leads to a resurgence of significant pain.

INTERVENTION: We have intensified the collaboration between our surgeons and the acute pain team nurse and anesthesiologists for patients who undergo scoliosis or pectus excavatum repair. Through this integrated approach, patients benefit from ongoing pain team care beyond the transition phase from PCA or epidural modalities to the end of PO medication at home.

RESULTS: Prospectively acquired data from this program will be presented. Daily drug use, pain scores, rate of decrease, side effects and level of activity will be discussed. The number of calls to surgery clinics, physicians and ward nurses made by the families after hospital discharge was significantly lower once the program was running.

CONCLUSION: We believe that in the case of surgical procedures followed by prolonged severe pain, it is not enough to achieve good pain control in the first few postoperative days.

\section{C19}

\section{MULTIMODAL INPATIENT PAIN TREATMENT IN CHILDREN - RESULTS OF A THREE WEEK PROGRAM} M Dobe $^{1}$, U Damschen ${ }^{1}$, B Reiffer-Wiesel ${ }^{1}$, C Sauer $^{1}$, B Zernikow ${ }^{2}$ ${ }^{1}$ IKP; ${ }^{2}$ Children;s Hospital Datteln - Witten/Herdecke University (IKP), Datteln, Germany

OBJECTIVE: In children and adolescents we investigated the impact of a 3 week inpatient multimodal pain therapy on subjective burden of life, pain intensity, and number of days off school 3, 6, or 12 months later.

METHODS: At the beginning of therapy (inpatient setting), and 3, 6, and 12 months thereafter (outpatient setting) we collected the respective data using standardized questionnaires. For statistical analysis we used the Wilcoxon signed rank test. A $\mathrm{P}<0.05$ was regarded as statistically significant. RESULTS: 72 patients aged 7.5 to 18.2 Ys suffering daily pain entered the study, most of them being diagnosed with somatoform pain disease. After 3,6 , and 12 months, 65, 27, and 30 patients could be reevaluated. Mean pain intensity of the week before data acquisition was significantly less than at the beginning $(2.9,2.3,2.9$ vs 6.3$)$ as was the mean number of days off school due to pain during the four weeks period before each day of data acquisition $(1.8,1.5,1.4$ vs 9.2). Mean subjective burden of life was significantly less than at the beginning $(24.5,22.0,25.8$ vs 37.8).

CONCLUSION: Outpatient multimodal pain therapy has a sustained impact on children and adolescents suffering from chronic pain.

Supported by the Vodafone Foundation Germany 
C20

\section{CHRONIC PAIN IN ADOLESCENTS: EVALUATION OF REHABILITATION BASED ON BEHAVIOUR MEDICINE: A CASE SERIES}

LAhlqvist $^{1}$, R Wicksell ${ }^{2}$, G Olsson ${ }^{2}$

1Pain Treatment Service, ${ }^{2}$ Astrid Lindgren Children's Hospital, Stockholm, Sweden

BACKGROUND: Previous studies have shown that chronic and recurrent pain is common in school age children (Perquin et al, 2000,2001). The most common locations of pain are head, limb, and gut. Treatment is frequently unsatisfactory according to patients, families, and physicians.

The optimal treatment for these patients may be interdisciplinary cognitive behavioural therapy, with parent involvement, which focuses on pain management and rehabilitation to normal activity (Ecclestone et al, 2003). This paper describes a residential intense rehabilitation for long-standing pain based on an interdisciplinary cognitive behavioural therapy, eg, Acceptance and Commitment Therapy within a behaviour medicine perspective.

The rehabilitation was developed for adolescents who were not able to commute once a week to the hospital, instead patients and parents stayed for two weeks at the hospital and returned for a 3-day follow-up after 7 weeks.

AIM: The aim of this study was to evaluate if this rehabilitation was effective in improving functional ability in adolescents with idiopathic pain.

METHOD: Subjects were recruited by ordinary referral. A case series of twelve patients with long-standing pain were conducted. Participants were between 12 and 20 years old. The measures were administrated two weeks prior to intervention, two weeks and three months after. The measures included both daily registrations and self-estimated questionnaires. They measured functional ability, kinesiophobia and school absence.

In rehabilitation, exposure was the main intervention. Physical and social limitations, school absence, avoidance of planning activities and attitudes consisted important targets. Values, long-term consequences of avoidance, the meaning of thoughts and bodily sensation were emphasized.

RESULT: A substantial decrease in functional disability, kinesiophobia and school absence were seen. The data are being analysed and the result will be presented in the poster.

\section{C21}

\section{LUMBAR PLEXUS BLOCKADE IN CHILDREN}

\section{Lobias}

University of Missouri - Columbia, Missouri, USA

INTRODUCTION: There is growing interest in the use of peripheral blockade rather than epidural analgesia for unilateral surgery. There are limited reports regarding lumbar plexus blockade (LPB) in children. We present our experience with LPB and describe our modification of the adult technique.

METHODS: The pain service records and records from the anesthetic care of children during surgical trips to developing countries were reviewed. The placement technique was a modification of Winnie's technique with needle insertion more medial. A line was drawn parallel to the spinous processes through the posterior superior iliac spine (PSIS). A second line was drawn connecting the superior aspects of the iliac crests (intercristal line). An 18 gauge insulated Tuohy needle was inserted on the intercristal line halfway between the spinous processes and the point of intersection of the intercristal line and the line drawn parallel to the spinous processes. The transverse process was contacted and the needle walked off inferiorly. When a quadriceps muscle twitch was obtained, bupivacaine and clonidine were administered and a catheter threaded into the lumbar plexus space.

RESULTS: The cohort included 14 patients ( 5 to 11 years, 20 to $56 \mathrm{kgs}$ ). Ten patients received intermittent doses and 4 patients received a continuous infusion of local anesthetic. Median pain scores were $0-2,0-2$, and $0-2$ on days 1 to 3 . Ten patients received supplemental analgesia with ibuprofen (1-3 doses per day) and 3 patients received supplemental morphine (1-2 doses per day). The catheters were left in place for 67 to 78 hours. No complications were noted
CONCLUSIONS: Our experience demonstrates the feasibility of LPB for pelvic and femur surgery and confirms that a more medial insertion site should be used in children. These data add to the limited body of information regarding catheter placement for continuous infusions or intermittent dosing.

\section{C22}

PAIN MANAGEMENT PRACTICES IN EMERGENCY: INTERVENTIONS WITH PEDIATRIC NURSES AND PARENTS (PRELIMINARY RESULTS OF THE PAMPER STUDY)

$\underline{\text { S Le May }}{ }^{1}$, C Johnston ${ }^{2}$, M Choinière ${ }^{3}$, D Kudirka ${ }^{4}$, I Hubert $^{4}$, A Nolan $^{5}$, L Murray ${ }^{4}$, D Chalut ${ }^{4}$

${ }^{1}$ University of Montréal, Lorraine; ${ }^{2}$ McGill University; ${ }^{3}$ Montréal Heart Institute; ${ }^{4}$ Montréal Children's Hospital; ${ }^{5}$ Lasalle's Hospital, Montréal, Québec

BACKGROUND: Children's pain in emergency departments (ED) is not well managed. Medical doctors tend to underprescribe analgesics and nurses tend to underevaluate and undermedicate children's pain (Kim et al, 2003). OBJECTIVE: Verify the efficacy of tailored interventions with nurses and parents, on nurses' knowledge-practices and on parents' beliefs, regarding pain management, and also on children's pain scores.

METHODS: Retrospective and prospective (6-phase) study with a prepost design (nurses) and RCT (parents/children dyads - PCD).

SETTING: Pediatric ED of a university hospital.

SAMPLES: Review of 150 charts of children who presented with either acute abdominal pain, fracture, sprain, deep lacerations or burns. All nurses of the unit (55), $68 \mathrm{PCD}$ in each group (control and experimental). Children 4-15 yo with one of diagnoses precited.

INTERVENTIONS: Capsules on pain (nurses), booklet and bookmark (PCD).

INSTRUMENTS: Pain Management Experience Evaluation for chart reviews, Pediatric Nurses Knowledge and Attitudes Survey (PNKAS) on pain, Bieri or NRS for child's pain + distress levels, Pain Barriers Questionnaire for parents' beliefs,

RESULTS: Only 35\% of children (53/150) received an analgesic. $58 \%(87 / 150)$ of them had their pain documented, but nurses rated intensity on 3\% (3/87). 78\% nurses responded and had Mean PKNAS score of $71.7 \% \pm 12.5 \%$. Average level of pain and distress were similar between both groups of PCD. No significant statistical difference on parents' beliefs. CONCLUSIONS: Preliminary results show that nurses' knowledge on pain is moderately low, and that children's pain is still underreported and undermedicated. Also, interventions with parents were not effective at this phase of the study.

\section{C23}

THORACIC EPIDURAL PLACED UNDER GENERAL ANESTHESIA IN CHILDREN - IS IT SAFE?

R Efrat, L Kachko, I Ronen, C Platis, J Katz

Schneider Children's Medical Center of Israel, Petah Tikva, Israel

INTRODUCTION: Epidural analgesia is a popular tool due to its safety and high efficacy for perioperative pain treatment. Thoracic epidural anesthesia (TEA) is widely used for upper abdominal and thoracic surgeries. Inserting epidural catheter before inducing general anesthesia (GA) gives the opportunity to detect paresthesias and avoid neurological deficit. This is especially important for TEA. In children the epidural catheter is inserted under GA (except in teenagers) due to lack of cooperation of the child. Recently, there are many publications concerning risk/benefit ratio of performing TEA under GA versus inserting it from the lumbar or sacral approach and advancing it to the thoracic region.

We have compared technical difficulties during epidural catheter insertion and rate of postoperative complications between TEA and lumbar epidural analgesia (LEA) in anesthetized children.

METHODS: We compared 48 children with TEA to 127 children with LEA. Groups were matched for age, sex and type of surgery.

Epidural was inserted under GA before starting the surgery. The equipment used was Portex Paediatric Epidural Minipack with 18G short Tuohy needle for children up to 5 years and long Tuohy needle for older children. 
Loading medication was Bupivacaine or Ropivacaine and continuous drip of Ropivacaine, with or without Fentanyl.

RESULTS: The rate of technical complications was higher in LEA group. Bloody tap during thoracic epidural insertion was the only event $(2.1 \%)$. In the LEA group $7.9 \%$ complications were recorded (multiple pricks, bloody taps, unsuccessful catheter advancement). One case of unsatisfied analgesia was recorded in the TEA group (2.1\%). $7 \%$ was the rate of complications during postoperative infusion in the LEA group (mild motor block, unsatisfied analgesia, vomiting, pruritus). One LEA was discontinued due to uncontrolled pain of uncertain reason.

CONCLUSIONS: In our study the complication rate of TEA under GA was found to be lower than LEA. In our opinion, this controversial issue has to be checked in further controlled study.

\section{C24}

JUVENILE PAIN AMPLIFICATION SYNDROMES - IS MULTIDISCIPLINARY THERAPY THE ANSWER?

$\underline{\text { M Richter }}{ }^{1}$, M Offenbächer ${ }^{2}$, M Spamer ${ }^{1}$, A Schlosser ${ }^{1}$, R Häfner ${ }^{1}$, H Michels ${ }^{1}$

${ }^{1}$ German Center for Pediatric Rheumatology, GarmischPartenkirchen; ${ }^{2}$ Institute for Medical Psychology University of Munich, München, Germany

OBJECTIVE: Juvenile idiopathic musculoskeletal pain concerns an increasing number of patients. The intensity of pain leads to the term "pain amplification syndrome". Laboratory results are normal and further investigations exclude other diseases that could reasonably explain the pain.

PATHOPHYSIOLOGY: Amplification is assumed to take place in the central nociception system with a winding up mechanism and subsequent central sensitization for pain.

SUBGROUPS: Pain amplification syndromes can be divided into localized or widespread pain and show a primary or secondary disease course.

PATIENTS AND METHODS: We have a three week inpatient program and present a cohort of 165 patients with 135 girls and 30 boys mainly 12 to 15 years old. $64 \%$ reported pain for at least two years with a disease onset under 12 years.

165 patients answered a questionnaire before therapy. Directly after therapy they evaluated the treatment. A postal follow-up questionnaire was addressed to 115 patients $6-12$ months after discharge and answered by 85 patients (74\%).

THERAPY: After clinical assessment by a pediatrician the multidisciplinary therapy is focused on physiotherapy, occupational therapy, psychological intervention, social education, experienced nursing care, reintegration into school routine and supplementary therapy.

RESULTS: $81 \%$ of our patients showed encouraging improvements at time of discharge. 85 patients who answered the follow-up questionnaire showed significant improvements in pain intensity and duration, attendance at school and school sports and activities of daily life. A significant decrease was seen in medications, doctor visits and hospital stays.

CONCLUSION: The intense pain severely limits the children and leads to desocialization. Countless doctor visits and hospital stays burden the social and health care system. Finally the family and the child are overwhelmed by the disease.

Our daily experience and data on 165 children show the enormous limitation due to pain amplification syndromes but also encouraging results of a multidisciplinary treatment.

\section{C25}

\section{GROUP WORK IN YOUNG PEOPLE WITH HEADACHE \\ T Murphy}

Department of Psychological Medicine, London, United Kingdom

BACKGROUND: Chronic headache is a common problem in childhood. Recent studies have indicated that children with headache usually become adults with headache. Considering the longevity of headaches, learning life-long skills in headache management during childhood are important. Although pharmacological approaches remain the main treatment for frequent and/or severe headaches, many families find medication alone to be inadequate. Generally, group interventions in
D . DO NOT COPY

headache management using psychological approaches have mixed results, depending on the focus of the intervention and the outcome measure used to evaluate it. One of the limitations of the evaluation of headache treatments in general is that the outcome measure is usually focused on the frequency of headache. Few studies have evaluated how the young person is affected by their headache.

METHOD: Eight patients (four boys and four girls; age 11-15) with a diagnosis of migraine/chronic daily headache participated in six monthly sessions. Each session focused on an individual approach of headache management and enhancement of quality of life. Individual sessions focused on psychoeducation about headache, relaxation, imagery, stress management and management of headaches at school. Groups lasted 90-minutes and were facilitated by a clinical psychologist. A single-group session was attended by parents. Measures relating to the affects of headaches on the patient's life were completed pre- and postintervention by five patients.

RESULTS: The rating scales indicated that four of the five patients reported that they could manage their headaches better following the group and that the headaches had a lesser affect on their lives. Feedback from patients showed that they valued learning strategies to cope with headaches in addition to meeting other people with headache.

CONCLUSIONS: A group intervention was designed for young people with chronic headache drawing on the broad literature in this field. The current evaluation is limited by sample size but suggests that such interventions may have a positive affect on quality of life.

\section{C26}

\section{VIRTUAL REALITY AS A DISTRACTION TECHNIQUE FOR CHILDREN DURING MINOR MEDICAL PROCEDURES IN A PEDIATRIC EMERGENCY DEPARTMENT}

B Lange ${ }^{1}$, M Williams ${ }^{1}$, I Fulton ${ }^{1}$, M Craigie ${ }^{2}$

${ }^{1}$ University of South Australia; ${ }^{2}$ Flinders Medical Centre, Adelaide, Australia

AIMS: Nonpharmacological strategies for managing procedural pain have been reported to reduce pain and distress. The aim of this study was to determine if Virtual Reality (VR) is an effective distraction technique for children undergoing minor medical procedures in a Pediatric Emergency Department compared to watching an animated movie.

METHODS: Using a block randomized design, children (6-17 years) undergoing minor medical procedures (blood sampling, intravenous cannulae insertion, suturing) were offered either VR or an animated movie excerpt as a distraction technique. Pre, post and maximum scores were received for children's self-report pain (coloured analogue scale) and anxiety (facial affective scale). Behaviour was scored using the brief behavioural distress scale. Children completed a questionnaire about enjoyment/interest in the intervention. Parents and staff scored their perception of the child's pain and anxiety pre, during and postprocedure and their perception of the technique's effectiveness.

RESULTS: Eighty eight subjects $(51 \mathrm{M}, 37 \mathrm{~F}$, mean age $11.3 \pm 2.9)$ have been recruited $(V R=44$, movie $=44)$. Preliminary analysis suggests no difference in self-reported pain or anxiety between techniques, however children using VR displayed significantly lower behavioural distress. In terms of level of enjoyment, interest/involvement and interaction, VR scored significantly higher than the animated movie. No significant difference in parent or staff's perception of the child's pain and anxiety existed, however, VR was perceived to provide more effective distraction by both staff and parents. Final data analysis is expected to be completed by the end of January 2006.

CONCLUSIONS/IMPLICATIONS: These preliminary findings suggest that VR is at least as effective as watching an animated movie in reducing pain and anxiety in children during minor medical procedures. The lack of adverse effects suggest that VR may be employed within. 


\section{C27}

A NEW ANALGESIC STRATEGY BY ACETAMINOPHEN (APAP): STUDY OF AN INITIAL LOADING DOSE

R Ricard $^{1}$, L Lassauge ${ }^{2}$, A Alibeu ${ }^{3}$, F Fournier-Charriere ${ }^{4}$, P Pons ${ }^{5}$

${ }^{1}$ Centre de la Douleur CHU Lapeyronie, Montpellier; ${ }^{2}$ Consultation douleur enfant CHU Saint-Jacques, Besancon; ${ }^{3}$ Centre de la douleur enfant $\mathrm{CHU}$ Michallon, Grenoble; ${ }^{4}$ Service de pediatrie CHU Bicetre, Le Kremlin Bicetre; ${ }^{5}$ Pharmacologie clinique,

GH Cochin - Saint Vincent de Paul, Paris, France

The evaluation of a new antipyretic and analgesic APAP dosing schedule has been in progress after revisiting APAP pharmacokinetics (PK) and pharmacokinetic-pharmacodynamic (PK/PD) relationship between antipyretic effect and APAP plasma concentration. There was a lag time in maximal effect of APAP ranging between 7 to 20 hours related in part to the time to obtain steady-state concentrations. Furthermore there was another 1 to 2 hour lag time in the time course of maximal antipyretic effect compared to maximal plasma concentration.

To decrease this lag time, the use of an initial APAP $30 \mathrm{mg} / \mathrm{kg}$ loading dose (twice a usual dose), followed by the usual $15 \mathrm{mg} / \mathrm{kg} / 6 \mathrm{~h}$ maintenance schedule has been suggested.

A controlled clinical trial in febrile children demonstrated a single $30 \mathrm{mg} / \mathrm{kg}$ (loading dose) was superior to a $15 \mathrm{mg} / \mathrm{kg}$ single dose. Preliminary results of a repeated-dose trial confirm this finding.

The present study intended to demonstrate the analgesic superiority and the good clinical and biological safety of an initial $30 \mathrm{mg} / \mathrm{kg}$ loading dose. METHODOLOGY: Double-blind, multicenter, parallel-group, randomized clinical trial comparing postoperative analgesic efficacy of oral APAP after an initial $30 \mathrm{mg} / \mathrm{kg}$ loading dose versus a $15 \mathrm{mg} / \mathrm{kg}$ dose both followed by repeated $15 \mathrm{mg} / \mathrm{kg} / 6 \mathrm{~h}$ doses in 1 to 6 year-old children . Amount of rescue analgesia with nalbuphine was recorded. Anesthesia protocol combined a general to a locoregional anesthesia. Initial dose of APAP was administered in postanesthesia care unit. Pain was measured (VAS, CHEOPS) between 0.5 and 24 hours post surgery. Liver enzymes and acetaminophen plasma concentrations were recorded 24 hours after dosing. Adverse events were recorded.

RESULTS: 68 children undergoing various surgical procedures were included. Excellent clinical and liver (biological) safety was reported in both groups. Comparison of analgesic effects is ongoing.

CONCLUSION: Analgesic superiority and tolerance of an initial acetaminophen $30 \mathrm{mg} / \mathrm{kg}$ loading dose is currently being evaluated.

\section{C28 \\ IMPROVEMENT OF PAIN DURING ANAL DILATIONS IN INFANTS}

C Wood, O Perrusson, A Pasturel, N Duparc, L Teisseyre, S Evrot, Y Aigrain, T Moreaux, B Chaumont, V Hamelin, J Poirot, M Fournier, K Morvan, S Roux, S Fiquet, J Biraud, M Williamson Hôpital Robert Debré, Paris, France

Babies with Hirschsprung disease and anorectal malformations need regular anal dilations, often performed without analgesics.

Uneasiness was expressed by the nurses and psychologist around this procedure because of the pain, the way the parents went through this, and the symbolic representation of the gesture, and improvements seemed necessary.

OBJECTIVES: Improve the management of babies needing anal dilation. METHODS: Three steps were taken:

1) A working group discussed what steps had to be made, suggestions for treatment and management.

2) 19 children were followed during one year at home and at hospital and assessed during the procedure.

3) A "satisfaction inquiry" was sent to the surgical team.

RESULTS:

a) 271 dilations were assessed during the year: $57,2 \%$ were considered unpainful according to neonatal scales, done by parents or carers. $41,3 \%$ were considered painful. 1, 5\% were not assessed

b) Satisfaction Inquiry: most of the team found improvements which will be discussed.
CONGU, DO NOT COPY

CONCLUSION: The surgical team was satisfied in improving this procedure.

The study is being continued to:

- Distinguish the differences in pain between anoplasty and fistula

- Reflect on the indications and the way the procedure is realized

- Improve the way the parents are accompanied.

\section{C29}

\section{AN INTERVENTIONAL THERAPY DURING PAINFUL PROCEDURES: USE OF NITROUS OXIDE INHALATION DURING INTRA-ARTICULAR INJECTIONS IN CHILDREN WITH JUVENILE IDIOPATHIC ARTHRITIS}

T Holmberg' ${ }^{1}$, S Berg ${ }^{2}$, Brunsson $^{1}$, I Wester ${ }^{1}$, E Winsö ${ }^{2}$, R Stangebye-Nielsen ${ }^{2}$

${ }^{1}$ Department of Anaesthesia and Intensive Care; ${ }^{2}$ Department of Pediatrics, Gothenburg, Sweden

BACKGROUND: Children diagnosed with juvenile idiopathic arthritis, JIA, are often given intra-articular cortisone injections as treatment in an acute phase of the disease. Many children consider those injections painful and scary. Different methods to reduce pain are available: General anesthesia, EMLA® or infiltrative anaesthetics. Midazolam is also used. Within a pilot project at the Queen Silvia Children's Hospital the analgesic and sedative effect of nitrous oxide inhalation (50\% N2O/50\% O2) was evaluated during intra-articular injections in children.

METHODS: Children diagnosed with JIA aged 5-18 years were included. They were premedicated $2 \mathrm{~h}$ before the injection with locally applicated EMLA® and oral paracetamol and NSAID. A nurse administered nitrous oxide 3 minutes prior and during the injection using a verbal method for distraction called GAN; Guided Association and Nitrous oxide. Pulse oximeter was used for supervision. The childrens subjective experience of pain and the emotional component before, during and after procedure was evaluated. Two validated scales were used: Colored analogue scale (CAS, graded 0-10) and facial analogue scale (FAS, graded 0-1) respectively.

RESULT: 41 procedures were performed, 37 of those were completed. Median scores decreased for both pain $(2-1,4-0)$ and emotional component $(0,67-0,37-0,37)$ during injection compared to before the procedure. This was statistically significant $(\mathrm{P}<0.05)$ for the emotional component. After 37 of 41 procedures the child would like to have nitrous oxide inhalation in case of future intra-articular injection. We did not note any serious or irreversible side effects.

CONCLUSION: Nitrous oxide inhalation with 50\% N2O/50\% O2 during painful procedures as intra-articular injections is a good alternative to achieve analgesia and sedation according to the results and the children themselves. Premixed nitrous oxide can be administrated by nurses after education. Less patients will have to undergo general anesthesia which is both time sparing and cost-effective.

\section{C30}

\section{PAINFUL PROCEDURES IN THE PEDIATRIC EMERGENCY DEPARTMENT: A PILOT STUDY OF A DVD-BASED INTERVENTION FOR CHILD PAIN AND ANXIETY}

$\underline{\text { E Moon }}^{1}$, P McGrath ${ }^{1,2}$, E Fitzpatrick ${ }^{3}$, D Sinclair ${ }^{1,2}, C_{\text {Chambers }}{ }^{1,2}$ ${ }^{1}$ Dalhousie University; ${ }^{2}$ IWK Health Centre, Halifax, Nova Scotia Venepunctures (needles) are the most commonly reported painful procedures in the pediatric emergency department (ED) setting (Chambers et al, 2003). Short-acting analgesic and sedative medications are not appropriate for minor but frequent procedures such as venepunctures because they require extra personnel, often require having an IV in place and can have negative side effects. Topical anesthetics are also problematic because they are costly and require up to an hour of advance notice. As a result, there is a need for an inexpensive and quickly administered nonpharmacological method to reduce pain for minor procedures such as venepunctures in the pediatric ED. In the current pilot study, we examined whether the provision of a DVD-based intervention program in distraction and relaxation techniques reduced venepuncture-related child pain and anxiety in the ED. Two short DVD's were used in the intervention and all participants received the intervention. The first DVD contained instructions 
for parents and children on how to reduce pain and anxiety associated with venepunctures. The second DVD was an animated short film that was used as a distraction tool. The primary outcome measures were child self-report of anxiety and child self-report of pain. The mean child anxiety ratings was $3.7(\mathrm{SD}=3.1)$ on a numerical rating scale. The mean of the child pain ratings was $3.2(\mathrm{SD}=2.3)$ on the Faces Pain Scale-Revised (Hicks, von Baeyer, Spafford, van Korlaar \& Goodenough, 2001). The mean pain rating was on the borderline of our predetermined cut-off score for treatment efficacy; however, the small sample size and lack of control group in the current study prevent us from drawing any firm conclusions about the efficacy of our intervention program. A randomized controlled trial of the intervention program is currently underway.

\section{C31}

\section{INTEGRATING COMFORT POSITIONING INTO PATIENT CARE - WARNING: NEW PROTOCOL CAN BE HABIT FORMING}

\section{Kaczka}

Shriners Hospital for Children - Spokane, Spokane,

\section{Washington, USA}

Beginning a new protocol of patient care can be challenging for all staff. Although patient care staff desire to provide the best care possible to their pediatric patients and families, it is natural for team members to feel reluctant in changing years of practice. This is especially true when it comes to hands-on patient care. Asking staff to re-examine years of procedural techniques and to implement new skills and thought processes can be met with opposition. Through a systematic method, Shriners Hospital for Children - Spokane has worked successfully in integrating Comfort Position protocol into patient care. Because these new techniques were integrated in a methodical, nonthreatening approach, staff have integrated the practice of Comfort Positions into daily care.

This presentation outlines the steps taken over a two-year period to effectively integrate this practice in delivering care and ultimately reducing procedural stress for patients and their families. These steps included:

- Recreational Therapy/Child Life staff introduced the Comfort Position concept to the Pain Management Team and Medical Staff.

- Upon the committees' recommendations and with their support, a national speaker was invited to the hospital to present Comfort Position practices to all staff.

- Recreational Therapy/Child Life developed a CD ROM training program responsible for educating patient care staff to the theory in detail. - All new patient care employees are required to meet individually with a Recreational Therapist/Child Life Specialist for individualized education and discussion.

- A mandatory hands-on competency program was developed for patient care staff to demonstrate the ability to use Comfort Position protocol for phlebotomy, catheterization and intravenous therapy. Large dolls and staff members' children are utilized for this hands-on competency and practice. Through these steps, Comfort Position practices are being integrated into patient care, which promotes more parental involvement in procedures and can decrease a child's trauma.

\section{C32}

\section{A TREATMENT APPROACH TO CHRONIC AMPLIFIED MUSCULOSKELETAL PAIN IN PEDIATRICS \\ L Brake, K Dholakia, L Giordano \\ Children's Hospital of Philadelphia, Philadelphia, \\ Pennsylvania, USA}

BACKGROUND: At The Children's Hospital of Philadelphia (CHOP) the term Reflex Neurovascular Dystrophy (RND) describes the amplified musculoskeletal pain syndromes treated in the RND Program, which provides a holistic approach toward the treatment of pain. The program addresses physical symptoms and psychosocial issues which are dominant areas of concern in the patients' lives. This study analyzed changes in functional skills using the Bruininks-Oseretsky Test of Motor Performance (BOTMP) and pain scores after an average of $2-3$ week participation in the RND program.
METHODS: A retrospective quasi-experimental, one group pretestposttest design was used. Medical records and daily exercise forms were used to obtain pre- and postpain and BOTMP scores. The BOTMP is a standardized test of physical function for children aged 4 to 14 years. Thirty-eight medical records were obtained for subjects who participated in the program between April 2003 and January 2005. Data was analyzed using a paired t-test at $\alpha=0.05$ to determine significance.

RESULTS: Data was analyzed for three groups, all children who participated in the program $(n=38)$, children who fell within the target age range for the test $(n=13)$, and children who were over 14 years of age $(n=25)$. The results of this study indicate statistically significant improvements in global motor function and decrease in pain scores, recorded at initial exam and discharge for all groups.

CONCLUSIONS: Children with RND demonstrated improvements in their physical function along with a decrease in their pain scores pre- and postparticipation in CHOP's RND program. Intense physical therapy and occupational therapy which include activities to increase strength and endurance, desensitization, home exercise programs and timed activities with daily goals combined with a holistic approach are effective as a treatment approach for RND.

\section{C33}

\section{THE USE OF ALFENTANIL DURING CHEST TUBE REMOVAL AFTER PEDIATRIC CARDIAC SURGERY IN A NURSING WARD}

E Kokinsky ${ }^{1}, \AA ̊$ Kornegård-Bark ${ }^{2}$, E Bratt ${ }^{2}$

${ }^{1}$ Department of Anaesthesia and Intensive Care; ${ }^{2}$ The Paediatric Cardiac Centre, The Queen Silvia Children's Hospital, Sahlgrenska University Hospital, Göteborg, Sweden

BACKGROUND: Pain during chest tube removal can be intense and stressful. Alfentanil is a rapid and short-acting opioid suitable for short painful procedures. It has a more pronounced respiratory depressive effect than morphine. The aim of the study was to evaluate the effect of alfentanil during chest tube removal in children after cardiac surgery in a nursing ward.

METHODS: An anesthetist was present during the procedure. If the child was judged to be anxious midazolam $0.1 \mathrm{mg} / \mathrm{kg}$ iv was given before the bandage was removed. Alfentanil 10 microgram $/ \mathrm{kg}$ iv was administered 30-60 seconds before the tube was removed. Pain/ discomfort was assessed by using the Objective-Pain-Scale (0-1 year) or Toddler-PreschoolerPostoperative-Pain-Scale ( $>$ one year) before, during and after the procedure. The pain assessment scores were categorized as no or mild pain/ discomfort, moderate pain/discomfort and severe pain/discomfort. Breathing frequency and arterial oxygen saturation by means of a pulse oxymeter was monitored during the procedure.

RESULTS: Fifty-eight children 0-19 years (median 4 year) were included. Before the procedure, 47 children $(81 \%)$ were assessed to have no or mild pain/discomfort, 10 (17\%) moderate pain/discomfort and 1 (2\%) severe pain/discomfort. During the procedure the numbers were $32(55 \%)$, $20(35 \%)$ and $6(10 \%)$ respectively and after five minutes $50(86 \%)$, $8(14 \%)$ and none. In about half of the cases a decreased breathing frequency was noted one minute after administration of alfentanil. No child had apnea and no proceedings were required to stimulate breathing.

CONCLUSION: Alfentanil is a useful option during chest tube removal, although not completely eliminating pain/discomfort. No serious complications were noted.

\section{C34}

\section{USING ULTRASOUND TO IDENTIFY ANATOMIC LANDMARKS FOR PERIPHERAL NERVE BLOCKS IN PEDIATRIC PATIENTS}

\section{$\underline{\text { S Schrum, } M \text { Lewis }}$}

Nemours Children's Clinic, Jacksonville, Florida, USA

INTRODUCTION: Regional Anesthesia has continued to gain popularity for pediatric patients undergoing a variety of surgical procedures. However, since most anesthesia literature regarding the anatomic landmarks useful in performing peripheral nerve blocks is based on adult 
anatomy, there is a lack of knowledge regarding these landmarks in children. The purpose of this study was to use ultrasound technology to determine the external landmarks necessary to more accurately perform the axillary and femoral nerve blocks.

METHODS: This is an observational study.

After institutional review, twelve patients between 10-30 kilograms were enrolled into the study. Seven patients were in the 10-20 kilogram group and five patients were in the 20-30 kilogram group. The patients received general anesthesia for their surgical procedure. Using a Bard Access Systems Site rite 3 Ultrasound Scanner, the axillary and femoral nerves were visualized. An ultrasound picture of the nerve was taken with the site rite ultrasound camera. The data collected included the weight of the patient and the measured distance from skin to the femoral and axillary nerves.

RESULTS: The axillary and femoral nerves of patients in both weight groups were consistently identified using ultrasound technology and photographs were taken. The measured depth of the nerves correlated directly with the patients' weight.

CONCLUSION: The use of ultrasound techology can aid in the correct location of large peripheral nerves, femoral and axillary nerves. While peripheral nerve blocks were not performed in this study, it would be interesting to look at the success rate of these nerve blocks in children with the use of ultrasound guidance.

\section{C35}

\section{ANKLE BLOCK AND BIERS BLOCK PRODUCE EFFECTIVE, PROLONGED, PAIN RELIEF IN PRIMARY ERYTHROMELALGIA}

\section{Goddard, R Reaney, A Abbas}

\section{Sheffield Children's NHS Trust, Sheffield, United Kingdom}

Erythromelalgia is a rare condition characterized by red, hot, painful extremities. The pain is usually intense and burning in nature. We have reported this condition in a child (1); in common with other case reports effective pain relief was only achieved with major regional anesthetic blockade.

Yang et al (2) recently reported two missense mutations in the gene SCN9A in patients with primary erythromelalgia. These mutations affect the encoding of NAV 1.7, a voltage-gated sodium channel [alpha] subunit predominantly expressed in sensory and sympathetic neurones. SCN9A appears to play an important role in nociception and vasomotor regulation. In 2004 our patient, now aged 13, relapsed, with pain predominantly in his feet. Pain relief was initially achieved with a lumbar epidural. This was removed after twelve days because of back pain and pyrexia. Severe pain in his feet returned within six hours. The following day, left Biers block and right ankle block were performed under general anaesthesia. The Biers block produced significant pain relief and the ankle block complete pain relief. He went home the next day. He returned with recurrent symptoms two and three weeks later; a right Biers block and left Biers block, respectively, were performed under general anesthesia. He has since remained in remission.

Evidence is accumulating to support primary erythromelalgia being a genetically determined small-fibre axonopathy, resulting in abnormal excitability of sensory and sympathetic neurons. This evidence rationally supports the use of distal local anesthetic blockade, which we report to be effective.

\section{REFERENCES}

1. Harrison CM, Goddard JM, Rittey CD. The use of regional anaesthetic blockade in a child with recurrent erythromelalgia. Arch Dis Child 2003;88:65-6.

2. Yang Y, Wang Y, Li S, et al. Mutations in SCN9A, encoding a sodium channel alpha subunit, in patients with primary erythermalgia. J Med Genet 2004;41:171-4.
C36

\section{THE RECIPE CARD: INGREDIENTS FOR THE MOST OPTIMAL PEDIATRIC BURN TREATMENT}

\section{$\underline{\text { S Oppedisano, }} \underline{\text { S Alexander }}$}

The Hospital for Sick Children, Toronto, Ontario

Hospitalization after a major burn injury presents a time of acute stress and anxiety for children. The daily debridement and dressing change is often a very painful procedure, however, a necessary component to burn care. Children can experience overwhelming distress and lack of control around this procedure. To minimize distress around dressings, a Recipe Card was created by our pediatric burn unit. This poster presents this innovative nursing tool which outlines for the patient, nursing team and family those strategies, processes and/or comfort items that support an optimal dressing change for each child. It is a card that lists coping 'ingredients' that a child/family chooses for his/her own treatment and is updated daily to ensure it reflects the patient's changing needs. Ingredients can include items such as environmental preferences (ie, music), people who support the patient (ie, mom, uncle), comfort objects (ie, blanket) and suggestions regarding the process (ie, "Tell me what you are going to do before you do it"). In this way, the Recipe Card becomes the child's voice, speaking out about how the burn team can best help him/her cope with dressings and gives the child some control in a situation that inherently lacks control. Since its inception, the Recipe Card has promoted consistency and communication, empowered patients and families, and fostered our ability to provide family-centred care. It is a simple tool that can be adapted by any nursing team to help support a child that will undergo repeated traumatic and/or painful procedures.

\section{C37}

\section{WHAT PROBLEMS DO EPIDURAL CATHETERS CAUSE?}

\section{N Llewellyn, A Liley}

Birmingham Children's Hospital NHS Trust, Birmingham,

United Kingdom

BACKGROUND: In March 2003 several hospitals in the UK and Southern Ireland began collecting contemporaneous data on the incidence of clinical events associated with the insertion and subsequent management of epidural catheters. The aim of the audit was to enable clinicians to quote accurate levels of risk when discussing the technique with parents and children. Denominator age and insertion level data was collected and, in addition, information was collected on the following clinical events:

Postdural puncture headache

Peripheral nerve injury

Local anesthetic toxicity

Spinal cord insult

Pressure sores

Compartment syndrome

Drug error

Respiratory/cardiac arrest

Infection

Inadvertent spinal anaesthetic

RESULTS: 10,358 epidurals have been performed and 103 clinical events had been reported, $0.99 \%$ incidence.

Complete data is not yet available on 7 events.

The clinical event data was graded on a 1 to 4 basis $(1=$ most serious, 4 = least serious).

$\begin{array}{lllll}\text { Grade of event } & 1 & 2 & 3 & 4 \\ \text { Number } & 12 & 11 & 56 & 17 \\ \text { \% incidence } & 0.11 & 0.1 & 0.54 & 0.16\end{array}$

\begin{tabular}{|c|c|c|c|c|c|c|c|c|}
\hline $\begin{array}{l}\text { Number and \% } \\
\text { incidence of events }\end{array}$ & $\begin{array}{c}1 \\
\text { No }\end{array}$ & $\begin{array}{r}1 \\
\%\end{array}$ & $\begin{array}{c}2 \\
\text { No }\end{array}$ & $\begin{array}{r}2 \\
\%\end{array}$ & $\begin{array}{c}3 \\
\text { No }\end{array}$ & $\begin{array}{l}3 \\
\%\end{array}$ & $\begin{array}{c}4 \\
\text { No }\end{array}$ & $\begin{array}{l}4 \\
\%\end{array}$ \\
\hline \multicolumn{9}{|l|}{ Postdural puncture } \\
\hline headache & 1 & 0.009 & & & 5 & 0.048 & & \\
\hline Peripheral nerve injury & 2 & 0.019 & 6 & 0.057 & 1 & 0.009 & & \\
\hline Local anesthetic toxicity & & & 2 & 0.019 & & & & \\
\hline Spinal cord insult & 1 & 0.009 & & & & & & \\
\hline
\end{tabular}


Pressure sore Compartment syndrome Drug error Respiratory/cardiac arrest Infection $4 \quad 0.038$ 10.009 $3 \quad 0.028$

Inadvertent spinal anesthetic

$0.048 \%$ of children $(n=5)$ who had a grade 1 event continued to have difficulties one year after the insertion of the epidural catheter.

As a result of this work, practice in individual institutions has changed over the audit period. It may also be possible to develop guidelines on the investigation and management of some of these events.

\section{C38}

\section{ADVERSE NEUROLOGICAL EVENTS FOLLOWING EPIDURAL PLACEMENT IN ANESTHETIZED CHILDREN}

IRose $^{1,2}$, L Maxwell ${ }^{2}$, A Ganesh ${ }^{2}$, G Cucchiaro ${ }^{2}$,

R Cuy $^{2}$, T Dimmagio ${ }^{2}$ M Robinson ${ }^{3}$

${ }^{1}$ University of Pennsylvania, School of Medicine, ${ }^{2}$ Children's Hospital of Philadelphia, Philadelphia, Pennsylvania; ${ }^{3}$ Cincinnati Children's Hospital Medical Center, Cincinnati, Ohio, USA

Few studies regarding the safety of placing epidural catheters in anesthetized children exist (1). We designed a Pain Management Service Registry (PMSR) to provide the Pediatric Pain Management Service (PPMS) with a means to track adverse events (AE) in this patient population. All AE are recorded prospectively in the PMSR by a member of the PPMS after making daily rounds on each patient. This ongoing study is approved by our IRB. The PMSR was queried from its inception on $12 / 1 / 2001$ through $1 / 11 / 2006$ for all entries of epidural catheter placement. There were 1153 epidural catheters placed under general anesthesia in children < 21 years old: 318 thoracic $(28 \%)$, 385 lumbar $(33 \%)$, and 450 caudal $(39 \%)$. No AE were reported in $698(60.5 \%)$ patients. Miscellaneous AE (ie, nausea, vomiting or pruritus) were reported in $380(33.0 \%)$ patients. Neurological AE $(n=79)$ were reported in $75(6.5 \%)$ patients and included: motor block or weakness $(n=25,2.2 \%)$; sedation $(n=11,1 \%)$; epidural site inflamation $(n=10,0.9 \%)$; muscle spasm or myoclonus $(n=7,0.6 \%)$; headache $(n=7,0.6 \%)$; paresthesias $(n=6,0.5 \%)$, Horner's syndrome $(n=6,0.5 \%)$; dysesthesia $(n=3,0.3 \%)$; postdural puncture headache $(n=1,<0.1 \%)$; back pain $(n=1,<0.1 \%)$; seizure $(n=1$, $<0.1 \%)$; and hysterical paralysis $(n=1,<0.1 \%)$. There were no spinal cord injuries or epidural hematomas. Two patients with dysesthesias and 3 patients with paresthesias were due to the surgical procedure and not epidural placement. All other neurological AEs resolved.

Review of our PMSR for patients who had epidural catheters placed during general anesthesia reveals transient neurological AEs are uncommon. No permanent neurological complications occurred which could be attributed to epidural placement in anesthetized children. Further study is required to confirm the safety of this widely established practice.

\section{REFERENCES}

1. Giaufre E et al, Anesthesia and Analgesia 1996.

\section{C39}

\section{PERIOPERATIVE PAIN MANAGEMENT FOLLOWING THE NUSS PROCEDURE}

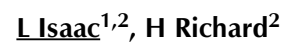

${ }^{1}$ Institute of Child Health and Great Ormond Street Hospital for Children, London, United Kingdom; ${ }^{2}$ The Hospital for Sick Children and University of Toronto, Toronto, Ontario

BACKGROUND: There is little documentation of the perioperative pain management for the minimally invasive technique of pectus excavatum repair, as described by Nuss (1), nor of the efficacy of pain control (2). We have undertaken a descriptive study of perioperative pain and analgesia after the Nuss procedure.

METHODS: All patients treated with the Nuss procedure since 2002 were prospectively followed by the pain service postoperatively, until pain was controlled by oral medications alone. Pain management technique, pain-related interventions, side effects and complications of treatment were documented. End points were defined as maximum daily pain scores, range of daily pain scores, daily morphine (or equivalent) consumption while using PCA or epidural, side effects of or complications of pain management techniques, and frequency of interventions due to pain as a surrogate for difficulty of pain management.

RESULTS: Records were obtained for 41 patients, primarily adolescent male, with all but one having primary repair of pectus excavatum. We describe maximum pain scores per day, and the trend of both maximum and minimum pain scores while treated with PCA and/or epidural. We describe daily morphine use, and side effects. We also describe the frequency and type of pain service interventions due to pain, side effects of or complications of pain management techniques.

DISCUSSION: Preliminary review suggests that most patients have moderate postoperative pain for three days, with only a few patients suffering prolonged, poorly controlled pain ( 5 days). However, this appears to be at the cost of regular 'rescue' analgesia usage despite continuous epidural infusion, frequent daily interventions by the pain service, and frequent morphine-related side effects.

\section{REFERENCES}

1. Nuss D, Kelly RE Jr, Croitoru DP, Katz ME. A 10-year review of a minimally invasive technique for the correction of pectus excavatum. J Pediatr Surg 1998;33:545-52.

2. Dilley AV, Cloyd H, Glass NL, Brown MF, Milewicz AL, Bloss RS. Pain management after minimally invasive pectus excavatum repair. Pediatr Endosurg Innovative Tech 2001;5:163-7.

\section{C40}

WHEN THE GOING GETS TOUGH: USE OF PALLIATIVE CARE SERVICES AT A CHILDREN'S HOSPITAL

M Middleton, S Combs, B McClain, J Drummond-Lewis

Yale University School of Medicine, New Haven, Connecticut, USA

INTRODUCTION: Children and families affected by illness and disease have complex physical and psychosocial needs that are not always appropriately addressed by the traditional health care team. Over 53,000 children in the United States die each year due to accidents, prematurity, congenital disorders, inherited disorders and acquired illnesses. Development and availability of in-hospital pediatric palliative care services are vital to ease physical and emotional suffering.

MATERIALS \& METHODS: The Dignity Project (DP) was developed for hospital-based patients. The goal is to provide a multidisciplinary approach to comfort care for children of all ages who face life-threatening illness. Through an interdisciplinary team approach, the emotional, physical, and spiritual needs of each child and family are addressed. The team includes representatives from medicine, nursing, psychology, child life, social work, chaplaincy, pharmacy and complementary and alternative medicine practitioners. Services are introduced by the DP and offered to the patient and family based on need and patient preference. Services include massage therapy, reflexology, reiki therapy, relaxation techniques, hypnotherapy, pain and symptom management, respite care, spiritual care, psychology services, and emotional support.

RESULTS: 32 patients with progressive illness were referred by primary care team and 23 patients participated. Referring teams included Hematology/Oncology, Neurosurgery, and Pediatrics. The service most requested was massage $(n=21)$, followed by Relaxation techniques $(n=20)$. The least requested service was hypnotherapy $(n=2)$. Indications for services included pain, anxiety, nausea, improved QOL and patient request. Reasons for decline of services included improvement in medical course, reliance on spiritual healing and anticipated transfer of care.

DISCUSSION: Many of patients referred to The Dignity Project received ongoing and regular services during hospitalization and upon subsequent admissions. Improvement in pain and symptom management was observed but not assessed for statistical significance. Comprehensive QOL indicators are needed to evaluate pediatric palliative care services. 
C41

\section{THE EFFECTIVENESS OF EARIY ANALGESIA IN CHILDREN PRESENTING WITH FRACTURES}

H Clare' ${ }^{1}$, L Buchanan ${ }^{2}$, M Sim², T Beattie ${ }^{2}$, L Aldridge ${ }^{2}$

${ }^{1}$ Medical School, University of Edinburgh; ${ }^{2}$ Royal Hospital for Sick Children, Edinburgh, Scotland

BACKGROUND: Fractures are a common, painful pediatric complaint, but this pain is frequently undertreated. This study aimed to assess the management of fracture pain in children, presenting to a pediatric accident and emergency department $(A \& E)$ within a pediatric teaching hospital. Children who require surgery on their fracture also have their pain management in theatre recorded. Efficacy of discharge analgesia in both groups was assessed. This new study allows a comparison to be made with a previous study carried out in 2002 which found that only $49 \%$ of children aged 3-13 years presenting with fractures or soft tissue injuries received analgesia. As a result of this, new practices were brought in to A\&E using Patient Group Directions, which allowed nurses to supply and administer paracetamol and ibuprofen and created a heightened awareness amongst staff of the need for analgesia for fractures and soft tissue injuries. METHODS: The pain scores of 70 children aged 3-13 with limb and clavicle fractures were assessed and the corresponding time and nature of $A \& E$ and theatre analgesia was recorded. Parents were telephoned at home to assess efficacy of their child's discharge analgesia.

RESULTS: Median pain scores on admission were 7.5 (range 3-10) for boys and 8.0 (range 2-10) for girls. In total $84 \%$ of the children had their analgesia status checked on admission, and $58 \%$ received analgesia. Only $39 \%$ of children were admitted for fracture reduction, and waited a mean time of 7 hours 22 minutes for surgery. The parent questionnaire found $16 / 17$ parents were satisfied with their child's pain management, and 15/17 parents found discharge analgesia satisfactory.

CONCLUSIONS: The pain management of fracture pain at the RHSC Edinburgh has been demonstrated to have improved greatly since 2002. Parents are very satisfied with their children's treatment.

\section{C42}

\section{OUTCOMES ASSOCIATED WITH PATIENT-CONTROLLED ANALGESIA (PCA) BY PROXY}

M Czarnecki, A Ferrise, M Sharp, K Pellmann, H Davies, S Weisman

Children's Hospital of Wisconsin, Milwaukee, Wisconsin, USA

INTRODUCTION: Patient-controlled analgesia (PCA) has been shown to be safe, effective, and superior to intermittent opioid dosing for pain management in children. However, not all children can operate a PCA independently due to age or developmental or cognitive delay. PCA by proxy, or parent/nurse-controlled analgesia (PNCA) has become more common; however, the safety and efficacy of this treatment modality have not been thoroughly studied.

PURPOSE: To describe pain scores, opioid usage, changes in treatment plans, and side effects associated with PNCA use for children with and without developmental delay at the Children's Hospital of Wisconsin. PROCEDURE: Data were collected from a retrospective review of a convenience sample of charts of children and adolescents admitted from 1999-2004.

ANALYSIS: SPSS 10.1 was used to determine means, frequencies, and ranges for demographic and descriptive data. Associations between variables were analyzed using Pearson correlations, analysis of variance and chi-square statistics.

RESULTS: Data were collected from 178 charts. Ages ranged from 11 days to 19 years. Seventy-one (40\%) children were identified as being developmentally delayed. Diagnoses were primarily surgical in nature $(84 \%)$. Pain was assessed on an average of 5.9 (day 1) to 3.1 (day 4) times per 12 hours shift. Pain scores were low, with a mean of $1.75( \pm 1.45)$ for children without developmental delay, and $1.04( \pm 1.19)$ for children with developmental delay. Morphine usage did not vary between groups, with an average morphine intake of $0.032 \mathrm{mg} / \mathrm{kg} / \mathrm{hr}$ for children with and without developmental delay. PNCA injections averaged 0.9 (day 1) to 0.5 (day 4) injections/hour with no difference between groups. Side effects
DINC, = DO NOT COPY

included nausea/vomiting $(13 \%)$; pruritis $(38 \%)$, and respiratory depression $(2.2 \%)$.

CONCLUSIONS: This retrospective study supports PNCA as a safe and effective method of pain management. The $2.2 \%$ occurrence of respiratory depression is comparable to previous literature reporting $1.7 \%$. Recommendations for best practice include appropriate PNCA dosing, frequent patient assessment, and pulse oximetry monitoring. No differences were found between children with or without developmental delay with regards to morphine use or pain scores. Results have led to a prospective study to further evaluate PNCA use in a more controlled manner.

\section{C43}

USE OF INTRATHECAL MORPHINE FOR POSTOPERATIVE ANALGESIA IN PEDIATRIC LAPAROSCOPIC RENAL PROCEDURES REDUCES THE LENGTH OF HOSPITAL STAY

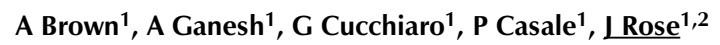

${ }^{1}$ Children's Hospital of Philadelphia; ${ }^{2}$ University of Pennsylvania, School of Medicine, Philadelphia, Pennsylvania, USA

INTRODUCTION: Intrathecal morphine (ITM) provides prolonged postoperative analgesia in pediatric patients and reduces the need for postoperative analgesics (1-3). At our institution, ITM is employed in laparoscopic renal procedures. We hypothesized low-dose ITM would reduce hospital length of stay (LOS) and postoperative complications.

METHODS: After IRB approval and informed consent, we reviewed 58 consecutive pediatric patients undergoing laparoscopic renal procedures. Variables recorded included age, procedures, ITM dose, LOS, and postoperative complications of vomiting (POV), respiratory depression, and pruritus.

RESULTS: 58 consecutive patients were studied. Patients in the ITM group $(\mathrm{n}=29)$ received $4-6 \mathrm{mcg} / \mathrm{kg}$ of preservative-free morphine intrathecally after induction of anesthesia. The non-ITM patients $(n=29)$ received morphine patient-controlled analgesia and/or intermittent morphine PRN. In the ITM group (mean age $6.2 \mathrm{yrs),} 16$ pyeloplasties and 13 ablative procedures were performed vs 17 pyeloplasties and 12 ablative procedures in the non-ITM group (mean age of $5.9 \mathrm{yrs}$ ). The ITM group had an average LOS of $26.2 \mathrm{hrs}$ (range 17-38 hrs) vs an average LOS for non-ITM group of $52.8 \mathrm{hrs}$ (range $26-111.2 \mathrm{hrs})$. ( $\mathrm{P}=0.048) \mathrm{POV}$ occurred in $13.7 \%$ of the ITM patients, and in $41.4 \%$ of non-ITM patients. The incidence of pruritus was $48.2 \%$ in the ITM group vs $37.9 \%$ in the non-ITM group. Respiratory depression was not identified in either group.

DISCUSSION: The use of ITM in laparoscopic renal procedures was associated with a reduction in LOS in our pediatric population. Similar findings have been described in adult surgical patients (4). Postoperative complications were not statistically different between ITM and non-ITM groups. However, the incidence of POV was lower in the ITM group. While we have examined a small population in a retrospective fashion, the outcomes observed warrant continued investigation of ITM in the pediatric surgical population.

\section{REFERENCES}

1. Kim AP, Cucchiaro G, Ganesh A. Anesthesiology 2005;103:A1352.

2. Tobias J. Anesth Analg 2004;98:956-65.

3. Goodarzi M. Paediatric Anaesthesia 1998;8:131-4.

4. Kong S-K, et al. Anesthesia 2002;57:1168-73.

\section{C44}

\section{CHRONIC HEADACHES IN CHILDREN: A MULTIMODAL APPROACH TO PAIN MANAGEMENT}

S Suresh, $\underline{\text { S Porfyris, }}$ M Jennings, V Sadanand

Children's Memorial Hospital, Chicago, Illinois, USA

AIM: The aim of this study was to examine our experience in managing patients who presented to the pediatric pain clinic with chronic nonmigraine headaches

METHODS: A prospective audit of our database was performed. All patients who presented to the pain clinic with a diagnosis of headache were included in the analysis. Descriptive statistical analysis was performed. 
RESULTS: A total of 98 visits were recorded on 89 patients in a period from 1994 to 2004 in the Aned from 7.0 years to 32 years of age with a mean of 16.2. The distribution is skewed to the right with $85 \%$ of the population between the ages of 10 and 21. Length of treatment by Pain Service ranged from same day discharge to 1644 days with a mean of 244 days. $25 \%$ of the patients had complete resolution of symptoms and only one patient reported no change in pain symptoms. There is a positive Spearman's correlation between age of the patient and the reported change in pain score although this correlation is not significant. Patients received a combination of treatment methods including nonpharmacological approaches including psychological intervention and physical therapy. Patients were provided pharmacological treatments including anticonvulsants as well as tricyclic antidepressants. Acupuncture was provided for some patients who were willing to use alternative medical approaches. If the above treatment was not providing pain relief, peripheral nerve blocks were performed. Supraorbital, supratrochlear, occipital blocks were offered to all patients who did not show improvements in symptoms with oral medication. Trigger point injections to the supraspinatus muscle as well as the trapezius was offered. Patients who received supraorbital and supratrochlear blocks, ie, those with frontal headaches had better resolution of symptoms. There were no adverse effects associated with use of nerve blocks. Maximum impact on pain index (MIPI) was 3.5 with occipital nerve blocks and 3.7 with a combination of supraorbital and supratrochlear nerve blocks. There was no difference in nerve block intervention for either frontal or occipital headaches.

DISCUSSION: A multimodal approach to chronic nonmigraine headaches can provide better pain relief in children. The use of peripheral nerve blocks in headache management is not prevalent in the pediatric population. We demonstrated that the use of peripheral nerve blocks along with conventional pain management techniques could facilitate pain relief. A potential for neuropathic pain in postsurgical patients may have contributed to their greater degree of relief with nerve blocks. Further work with placebo-controlled blocks may be needed to demonstrate the efficacy of local anesthetic blocks in patients with chronic nonmigraine headaches.

\section{C45}

\section{PEDIATRIC PALLIATIVE PAIN TREATMENT IN GERMANY}

\section{R Pothmann ${ }^{1}$, U Nerge ${ }^{2}$}

'Zentrum Kinderschmerztherapie Klinikum Nord; ${ }^{2}$ Kinder-Hospiz Sternenbrücke, Hamburg, Germany

In comparison with British palliative care (more than 40 pediatric hospices), the situation in Germany is quite different. During the last 10 years, however, 7 pediatric hospices and many specialized homecare services have been established. The necessity of pediatric palliative care has become part of the public discussion since pediatric oncology has been successfully developed. Nevertheless about 1600 children are dying every year because of different diseases, including cancer (1/3), metabolic diseases like neuronal ceroid lipofuscinosis (NCL) or adrenoleukodystrophy (ALD), muscular diseases like muscular atrophy, congenital malformations, infectious diseases like AIDS and (perinatal) traumata. A multiprofessional team of nurses, pediatricians and psychosocial workers is necessary to realize the complex challenge. Pain diagnosis and treatment plays a prominent role concerning the expectations of children and parents. More than $95 \%$ of the children can sufficiently be treated orally (metamizol, tramadol or combination of both) or by means of transcutaneous drug application (buprenorphine, fentanyl. Additionally, chloral hydrate or lorazepam often helps to overcome acute pain peaks and phases of unpleasantness. Our 2 years of experience handling the special conditions of pediatric pain therapy during the final stages of 28 children with life-limiting diseases in a German pediatric model hospice (Hamburg) will be discussed in detail.

\section{Pharmacology, Basic, Developmental, Clinical}

\section{D1} INFLUENCE OF MODULATORS ACTIVITY OF DRUG
METABOLIZING ENZYMES ON ANALGETIC EFFECT
KETOROLAC TROMETAMINE (KT) AT CHILDREN. D Dmytriiev

Vinnitsa National Medical University, Vinnitsa, Ukraine

Ketorolac trometamine (KT) is nonsteroidal anti-inflammatory drug with the strong analgetic effect, which is widely applied to treatment of painful syndrome in postoperative period at children. This drug quite often is combined with other pharmaceuticals, which can have properties of modulators activity of drug metabolizing enzymes and change analgetic effect KT. For example, dexamethasone, which is potent stimulator of UDP-glucuronosyltransferase expression.

The purpose of examination was to estimate influence of dexamethasone on analgetic effect $\mathrm{KT}$ at children.

17 children in early postoperative period are examinated. KT $(30 \mathrm{mg})$ introduced intramuscularly. Analgetic effect of KT estimated on dynamics of hydrocortisone level in blood plasma. In control group ( 8 children) are detected the established decrease of hydrocortisone medial concentrations after $12 \mathrm{~h}(191.1 \pm 84.3 \mathrm{nmol} / \mathrm{L})$ and $24 \mathrm{~h}(147.0 \pm 93,6 \mathrm{nmol} / \mathrm{L})$ since operation, in comparison with preoperative levels $(711.1 \pm 61.8 \mathrm{nmol} / \mathrm{L})$. In a basic group ( 9 children, which before operation administrated dexamethasone ( $1 \mathrm{mg} \mathrm{im})$ within 4 days and more) the plasma concentrations of a hydrocortisone also were reliably reduced in $12 \mathrm{~h}(184.3 \pm 92.2 \mathrm{nmol} / \mathrm{L})$ in comparison with preoperative levels $(782.2 \pm 94.8 \mathrm{nmol} / \mathrm{L})$, but at them in $24 \mathrm{~h}$ the magnification of hydrocortisone levels $(607.0 \pm 104.1 \mathrm{nmol} / \mathrm{L})$ was defined. Dynamics of hydrocortisone levels in blood in control group testifies to liquidation of painful syndrome at the majority of children within the first $12-24 \mathrm{~h}$ after operation, whereas in a basic group the duration of analgetic effect diminished in prestress reception of dexamethasone, which accelerates a metabolic inactivation KT.

\section{D2}

GENUINE, SUPPRESSED AND FAKED FACIAL EXPRESSIONS OF PAIN IN CHILDREN

A Larochette $^{1}$, C Chambers ${ }^{1}, K^{\prime}$ Craig $^{2}$

IIWK Health Centre, Dalhousie University, Halifax, Nova Scotia; ${ }^{2}$ University of British Columbia, Vancouver, British Columbia

Children's abilities to display faked and suppressed facial expressions of pain, relative to their genuine expression, and whether parents are able to distinguish between these expressions were investigated. Fifty healthy 8 - to 12 -year-olds ( 25 boys and 25 girls) submersed their hands in cold and warm water and were given different instructions about what to show on their faces. The painful cold pressor task (10 degree Celsius water) was used for the genuine and suppressed conditions and warm 30 degree Celsius water was used for the faked condition. Children were videotaped performing each facial display and the Facial Action Coding System (FACS) was used to provide detailed accounts of their facial expressions in each condition as well as during a baseline condition. Parents were subsequently asked to correctly identify each of the four conditions by viewing each video clip twice. Faked expressions of pain in children were found to show more frequent and more intense facial actions typical of a genuine pain expression, indicating that children were not able to successfully fake an expression of pain. Children's suppressed expressions, however, showed no differences from baseline facial actions, indicating that they were able to successfully suppress their expression of pain. Parents correctly identified the four conditions significantly more frequently than would be expected by chance. They were generally quite successful in detecting faked pain, but experienced difficulty differentiating among the other conditions. The results indicate that children are capable of controlling their facial expressions of pain when instructed to do so, but are better able to hide their pain than fake it. 


\section{D3}

\section{REPORTED PAIN COPING STRATEGIES IN CHILDREN AND ADOLESCENTS WITH SPINA BIFIDA}

C Clancy ${ }^{1}$, B Oddson ${ }^{2}$, P McGrath ${ }^{3}$

${ }^{1}$ Columbus Children's Hospital, Columbus, Ohio, USA;

${ }^{2}$ Laurentian University, Sudbury, Ontario; ${ }^{3}$ Dalhousie University, Halifax, Nova Scotia

BACKGROUND: The pervasive and chronic nature of pain associated with spina bifida demands the best possible management to protect quality of life. We hypothesized that the ways in which children with spina bifida cope with their pain will reflect both the resources they use to mobilize and the extent to which adequate pain management is provided.

OBJECTIVES: The primary goals of this study were to identify the repertoire of pain coping strategies used by children with spina bifida and to determine whether coping strategy use mediates the frequency and intensity of reported pain.

PARTICIPANTS: Our sample consisted of 68 children with spina bifida (30 males, 38 females) between the ages of 8 and 19 years (mean age 12 years, 8 months). Fifty-nine children $(87 \%)$ were diagnosed with myelomeningocele, six (9\%) with lipomyelomeningocele, and three (4\%) with lipomeningocele (4\%).

MEASURES: A semi-structured interview was used to obtain information about children's pain experiences, including frequency of pain, duration of pain (eg, hours, months, days) by location, and pain coping strategies in the week prior to the interview. Pain intensity was determined using the child self-report form of the Pediatric Pain Questionnaire. Pain intensity was assessed using the Pediatric Pain Questionnaire (PPQ).

RESULTS: Forty-nine children $(72 \%)$ had shunted hydrocephalus. Children reported that pain had a widespread impact on their lives, restricting their physical and social activities, impeding academic performance, disrupting sleep, and increasing school absenteeism. The most popular strategy was the use of over-the-counter analgesics, followed by rest/relaxation, distraction and the application of heat. Children reported using an average of two coping strategies in the 2 weeks preceding their interview; this number was not associated with frequency or intensity of pain, suggesting that children either have an insufficient number of strategies in their pain management repertoire, or that their existing strategies are ineffective at managing their pain. No relationships were found between the number and the types of pain coping strategies based on sex or age group.

CONCLUSIONS: Emphasis must be placed on disseminating information about the nature and prevalence of pain in children with spina bifida. There is also a potential role to teach a variety of effective pain management strategies to children and their caretakers.

\section{D4}

\section{THE ROLE OF PAIN IN REDUCED QUALITY OF LIFE IN CHILDREN WITH SPINA BIFIDA}

C Clancy ${ }^{1}$, B Oddson ${ }^{2}$, P McGrath ${ }^{3}$

${ }^{1}$ Columbus Children's Hospital, Columbus, Ohio, USA;

${ }^{2}$ Laurentian University, Sudbury, Ontario; ${ }^{3}$ Departments of Psychology, Pediatrics and Psychiatry, Dalhousie University, IWK Health Centre, Halifax, Nova Scotia

BACKGROUND: Children and adolescents with spina bifida face a variety of challenges to quality of life. They report significant episodes of pain, limited mobility, and both social and psychological barriers to participation in activities with peers.

OBJECTIVE: The goal of the present study was to demonstrate the direct and indirect impact of pain on various aspects of quality of life to highlight the importance of pain management for children and adolescents with spina bifida.

DESIGN/METHODS: A sample of 68 children (56\% female) between the ages of 8 and 19 completed the Varni/Thompson Pediatric Pain Questionnaire (PPQ), a semi-structured interview about their pain experiences, the Childrens Depression Inventory (CDI), the Nowicki-Strickland Locus of Control Scale for Children, and a pediatric measure of healthrelated quality of life (PedsQL).
DUP D. DONOF COPY

RESULTS: Health-related quality of life was shown to be systematically low in this group as compared to a reference sample of chronically ill children. It was negatively impacted by high reported frequency of pain and high ratings of current pain. Pain and low quality of life were strongly associated with CDI scores. Locus of control scores were not associated with quality of life or reported pain. Lesion level was not associated with any of the measures.

CONCLUSIONS: Unmanaged pain in children with spina bifida can have a substantial negative impact on overall functioning. We conclude that improving pain managment in children and adolescents with spina bifida is a priority because of its potential to substantially improve quality of life and well-being.

\section{D5}

\section{PREEMPTIVE ANALGESIA WITH TRAMADOL IN PEDIATRIC ANESTHESIA}

D Galante, G Pellico

Department of Anesthesia and Intensive Care, University Hospital "Ospedali Riuniti", Foggia, Italy

INTRODUCTION: Pain is a common complaint in children after surgery. There is uncertainty regarding the role of preemptive analgesia in preventing postoperative pain. The aim of our study is to evaluate efficacy and safety of preemptive analgesia with tramadol administered as a bolus vs continuous infusion in children undergoing abdominal and genitourinary surgery.

METHODS: 30 children classified as ASA I-II aged from 6 months to 6 years undergoing abdominal and genitourinary surgery were allocated to two groups: Group $\mathrm{Tb}(\mathrm{n}=15)$ received tramadol as a bolus $(1 \mathrm{mg} / \mathrm{kg})$, Group $\mathrm{Ti} \quad(\mathrm{n}=15)$ received continuous infusion of tramadol ( 150 microg $/ \mathrm{kg} / \mathrm{h}$ ) by elastomeric pump combined with bolus. Both of the groups received ondansetron $(0.10 \mathrm{mg} / \mathrm{kg})$. Each treatment was started at the induction of general anesthesia. Pain assessment was evaluated with Observer Pain Scale (OPS). Intraoperative HR, NIBP, MAP, SpO2 were monitored. PONV and additional doses of drugs were recorded.

RESULTS: No significants side effects were observed in any of the two groups and PONV were not statistically different between two study groups. HR, NIBP, MAP remained within the normal range. None of the children in Group Ti received additional analgesia before $24 \mathrm{~h}$. Three children of Group $\mathrm{Tb}$, in contrast, needed further drug administration postoperatively.

CONCLUSIONS: Preemptive analgesia with tramadol plus ondansetron seems to be effective and safe when it is administered in continuous infusion combined with bolus in children undergoing abdominal and genitourinary surgery.

\section{REFERENCES}

1) Umuroglu T, Eti Z, Ciftci H, Yilmaz Gogus F. Analgesia for adenotonsillectomy in children: A comparison of morphine, ketamine and tramadol. Paediatr Anaesth 2004;14:568-73.

2) Mikhel'son VA, Zhirkova IuV, Beliaeva ID, Stepanenko SM, Manerova AF, Butyleva OIu. Postoperative analgesia with tramal in newborn children using the method of continuous intravenous infusion. Anesteziol Reanimatol 2003;24-8.

\section{D6}

\section{ASSESSMENT OF QUALITY OF PARENT ADMINISTERED POSTOPERATIVE ANALGESIA IN CHILDREN UNDERGOING DAY CASE SURGERY: A COMPARISON OF THREE REGIMENS}

A Bhatia ${ }^{1}$, D Pawar ${ }^{2}$, R Subramaniam ${ }^{2}$, A Bhat ${ }^{3}$

${ }^{1}$ Department of Anaesthesia, Addenbrooke's Hospital, Cambridge, United Kingdom; ${ }^{2}$ Department of Anaesthesia, All India Institute of Medical Sciences, New Delhi, India; ${ }^{3}$ Department of Anaesthesia, Peterborough District Hospital, Peterborough, United Kingdom We conducted a randomized study for assessing quality of parent administered postoperative analgesia in 60 children (aged 1 to 8 years) undergoing day case lower abdominal or urological surgery under general anesthesia 
and caudal epidural block with $0.25 \%$ bupivacaine. The patients were randomized into three groups of 20 patients each to receive one of the following regimes of orally administered postoperative analgesia by their parents after discharge to home. Group P: paracetamol initial dose of $30 \mathrm{mg} \mathrm{kg}^{-1}$ followed by $20 \mathrm{mg} \mathrm{kg}^{-1}$; Group I: ibuprofen $20 \mathrm{mg} \mathrm{kg}^{-1}$; Group PI: combined paracetamol $10 \mathrm{mg} \mathrm{kg}^{-1}$ and ibuprofen $10 \mathrm{mg} \mathrm{kg}^{-1}$ per dose. Parents assessed their child's pain every 6 hours on the first 3 postoperative days on a three-point scoring system (1: no pain; 2: mild pain; 3: severe pain) and administered analgesia for a score $>1$. The number of analgesic doses required was similar in the three groups. Pain scores were slightly higher in Groups P and I compared with Group PI on the first postoperative day but the difference was not statistically significant. Recovery parameters, time to ambulation and play were similar in the three groups. Quality of sleep was good in the majority of patients in all groups. Parent satisfaction with the pain management protocol and all the three postoperative analgesic management regimes was high (90\%). We recommend the administration of paracetamol and ibuprofen combination in this surgical setting by parents to their children at home in the postoperative period on the basis of a protocol which includes details of objective assessment of pain.

\section{D7}

\section{ADDITION OF KETAMINE TO CAUDAL BUPIVACAINE FOR DAY-CASE PEDIATRIC LOWER-ABDOMINAL AND UROLOGICAL OPERATIONS}

\section{Sehgal $^{1}$, A Bhatia ${ }^{2}$, D Pawar ${ }^{1}$}

${ }^{1}$ Department of Anaesthesia, All India Institute of Medical

Sciences, New Delhi, India; ${ }^{2}$ Department of Anaesthesia, Addenbrooke's Hospital, Cambridge, United Kingdom

The use of ketamine as an adjunct to local anesthetics for caudal epidural analgesia has a proven value but its usage in day care patients has been questioned due to safety concerns. This randomized, double-blind study was conducted to evaluate the efficacy of ketamine in day care pediatric surgical patients undergoing lower abdominal and urological operations. Forty ASA I patients were divided into two groups after induction of general anesthesia. Caudal epidural injection was performed in all patients: patients in group B received only $0.25 \%$ bupivacaine while patients in group BK received $0.5 \mathrm{mg} \mathrm{kg}^{-1}$ ketamine along with $0.25 \%$ bupivacaine. The parents assessed the pain with the help of a simplified pain assessment scale for three days after the operation. A combination of ibuprofen and paracetamol $20 \mathrm{mg} \mathrm{kg}^{-1}$ of each as first dose and $10 \mathrm{mg} \mathrm{kg}^{-1}$ each for subsequent doses was administered by the parents for analgesia following pain assessment. The patients in group B had high incidence of pain at the time of discharge $(\mathrm{P}<0.001)$. Although not statistically significant, the patient in group $\mathrm{BK}$ required less number of postoperative analgesic doses. The time to full awakening, ambulation and demand for water were similar between the groups. No differences were observed in incidence of nausea, vomiting and urinary retention. This study shows that the addition of ketamine $0.5 \mathrm{mg} \mathrm{kg}^{-1}$ to $0.25 \%$ bupivacaine administered in caudal epidural space significantly reduced the pain at the time of discharge with minimal side effects.

\section{D8}

\section{A TASTE-TESTING STUDY IN HEALTHY CHILDREN TO INVESTIGATE PREFERENCE FOR IBUPROFEN SUSPENSIONS OR PLACEBO}

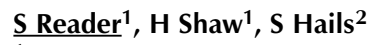

${ }^{1}$ Boots Healthcare International, Nottingham; ${ }^{2}$ Reading Scientific Services Limited, Reading, United Kingdom

AIM OF INVESTIGATION: To identify the extent to which the taste of ibuprofen is masked in commercially available ibuprofen suspension and to measure the influence of the 'ibuprofen taste' on children's preference in comparison with placebo.

METHODS: 151 children aged 4-7 years tasted 2 suspension samples: ibuprofen $5 \mathrm{mg} / \mathrm{mL}$ and matched placebo, in a randomized order. Children received a volume equal to the recommended over-the-counter dose of ibuprofen suspension ( $7.5 \mathrm{~mL}$ for $4-6$ year olds and $10 \mathrm{~mL}$ for 7 year olds).
DINC, DONOT COPY

After tasting each sample, children indicated how much they liked that sample on a $10 \mathrm{~cm}$ visual analogue scale (VAS) depicted with a 'sad face' at the beginning of the scale and a 'happy face' at the end, and described what they liked or disliked about the sample. After tasting both samples, children were asked whether they could distinguish between the samples and, if so, which they preferred, and why.

RESULTS: Mean (SD) VAS scores, measured from the 'sad face' end, were 6.78 (3.49) and 7.13 (3.42) for ibuprofen and placebo, respectively $(\mathrm{P}=0.38) .84 \%$ of children could distinguish between the samples and, of these children, $58 \%$ preferred placebo and $42 \%$ preferred ibuprofen $(\mathrm{P}=0.07)$. Preference for the placebo was driven by a perception of sweetness compared with the ibuprofen suspension.

CONCLUSIONS: There were no significant differences between ibuprofen and placebo in any parameter assessed, suggesting that the formulation effectively masks the taste of raw ibuprofen. The 'peppery taste' characteristic does not appear to be a significant factor in driving taste preference.

\section{D9}

NEONATAL PAIN EXPERIENCES ALTER THE BEHAVIOUR OF PARENTS WHEN THE CHILD IS IN PAIN

L Hohmeister, K Zohsel, C Hermann

Department of Neuropsychology and Clinical Psychology,

Central Institute of Mental Health, Mannheim, Germany

During the NICU stay of their child, parents perceive a distressing failure to protect their child from pain. This experience might alter the parents' later behaviour when the child is in pain, which in turn could influence the pain experience of the child via social modelling and operant conditioning. To test this hypothesis, questionnaire data on maternal behaviour when the child is in pain were collected from 19 preterm and 20 full term children with NICU experience and their mothers as well as from 20 full term control children without NICU experience and their mothers. In addition, the children's perceptual response to tonic painful heat was measured in the absence and in the presence of the mother and their painrelated cognitions were assessed via questionnaire. When tested, the children were between 9 and 14 years old. The mothers of the preterms reported significantly more solicitous responses when their child is in pain than the mothers in the control group. A similar (though statistically not significant) pattern was observed for the mothers of NICU full terms. In addition, there was a significant interaction between group and mothers presence in the perceptual response to tonic painful heat. While mother presence had a pain alleviating effect in the control group, this effect was lacking in both groups of NICU children. Furthermore, preterm children reported significantly more pain-related catastrophizing than controls. These results provide first evidence for long-lasting changes in the behaviour of the parents of NICU children, at least when these are born preterm. A greater level of solicitous responses on the part of mothers of NICU children may promote the child's experience of pain and may increase pain related catastrophizing in these children via social modelling and operant conditioning.

\section{D10 \\ QUANTITATIVE SENSORY TESTING IN CHILDREN WITH RECURRENT ABDOMINAL PAIN}

K Zohsel, J Hohmeister, C Hermann

Department of Neuropsychology and Clinical Psychology,

Central Institute of Mental Health, Mannheim, Germany

Recurrent abdominal pain (RAP) is one of the most common pain complaints in school-aged children. According to long-term follow-ups, children with RAP do not just "grow out of it", but have a higher risk to suffer from somatic symptoms in adulthood, too. The etiology of RAP is not yet fully understood. However, recent studies suggest that altered pain sensitivity as well as familial factors are involved.

We used quantitative sensory testing to study mechanical and heat pain sensitivity in 20 children with RAP ( 11 female, $M=10.7$ yr) and 23 healthy controls ( 10 female, $M=11.0 \mathrm{yr}$ ). Pain thresholds and measures of perceptual sensitization in response to repetitive or tonic noxious stimulation at both the abdomen and the thenar were assessed. In 
addition, the mother was either present or absent during measurement. Maternal behaviour when the child is in pain was assessed via questionnaire (mother and child rating).

Pain sensitivity at the abdomen did not differ between groups for any of the dependent measures. However, at the thenar, the children with RAP showed more habituation to tonic heat $(\mathrm{P}<0.05)$ and a trend towards less sensitization for repetitive mechanical stimuli $(\mathrm{P}=0.08)$. No significant effect of mother presence was found. Mothers of RAP children reported significantly less solicitous responses when their child was in pain than mothers in the control group $(\mathrm{P}<0.01)$. The children's rating of maternal pain-related behaviour did not differ between groups.

To summarize, no evidence was found for an increased pain sensitivity in RAP children. Rather, these children may be less prone to perceptual sensitization at a non-pain site. Mother behaviour during pain episodes of the child seems to be altered in the RAP group which may partially reflect changes in maternal behaviour when pain occurs on a recurrent basis. Implications for the understanding of RAP will be outlined.

\section{D11}

\section{HEMODYNAMIC CHANGES DURING INTRAOPERATIVE DEXMEDETOMIDINE ADMINISTRATION: SEVOFLURANE VERSUS DESFLURANE ANESTHESIA}

\section{LTobias}

University of Missouri, Columbia, Missouri, USA

INTRODUCTION: Dexmedetomidine decreases the incidence of emergence agitation following sevoflurane or desflurane anesthesia. As there may be synergistic hemodynamic depression when dexmedetomidine is combined with inhaled anesthetic agents, we prospectively compared the hemodynamic effects of dexmedetomidine in pediatric patients anesthetized with desflurane or sevoflurane.

METHODS: Following endotracheal intubation, anesthesia was maintained with sevoflurane or desflurane. Dexmedetomidine $(0.5 \mu \mathrm{g} / \mathrm{kg})$ was administered over 5 minutes. Heart rate (HR), systolic and diastolic blood pressure $(\mathrm{sBP}, \mathrm{dBP})$ were recorded prior to dexmedetomidine, at the completion of the dose, and at 5 and 10 minutes after dexmedetomidine. Statistical analysis included Donnett, ANOVA, and a nonpaired t-test. RESULTS: The study cohort included 50 patients (22 sevoflurane, 28 desflurane). In both groups, there was a HR decrease at 5, 10, and 15 minutes following dexmedetomidine. In patients anesthetized with sevoflurane, the $H R$ values at $0,5,10$ and 15 minutes were $137 \pm 23$, $115 \pm 20,109 \pm 21$, and $104 \pm 19$ beats/min. In patients anesthetized with desflurane, the HR values were $142 \pm 19,127 \pm 20,124 \pm 2$, and $119 \pm 19$ beats/min. The lowest HR was $103 \pm 20$ beats/minute with sevoflurane vs $116 \pm 19$ beats/minute with desflurane $(\mathrm{P}=0.0231)$. The lowest sBP following dexmedetomidine in patients anesthetized with sevoflurane was $92 \pm 13 \mathrm{mmHg}(\mathrm{P}<0.01$ compared to baseline of $104 \pm 16 \mathrm{mmHg})$. The lowest $\mathrm{sBP}$ in patients anesthetized with desflurane was $97 \pm 11 \mathrm{mmHg}$ $(\mathrm{P}<0.01$ compared to baseline of $108 \pm 11 \mathrm{mmHg}$ and $\mathrm{P}=\mathrm{NS}$, desflurane vs sevoflurane).

CONCLUSION: Although there were no clinically significant hemodynamic changes following the administration of dexmedetomidine to patients anesthetized with either sevoflurane or desflurane, the decrease in HR was greater in patients anesthetized with sevoflurane. The potential for HR decreases should be considered in subpopulations who may not tolerate this relative bradycardia.

\section{D12 \\ DEXMEDETOMIDINE CONTROLS WITHDRAWAL FOLLOWING PROLONGED USE OF OPIOIDS AND BENZODIAZEPINES IN INFANTS}

\section{$\perp$ Tobias}

University of Missouri - Columbia, Missouri, USA

INTRODUCTION: Tolerance, physical dependency, and withdrawal may occur following the prolonged use of opioids and benzodiazepines for sedation in the Pediatric ICU patient. We report our experience with the use of dexmedetomidine to treat withdrawal following sedation during mechanical ventilation in infants.
METHODS: We retrospectively reviewed the pharmacy records and identified infants who had been treated with dexmedetomidine for acute withdrawal. Although the data were retrieved retrospectively, treatment of these patients was by a specific protocol which included both dexmedetomidine dosing (loading dose of $0.5 \mu \mathrm{g} / \mathrm{kg}$ over 5-10 minutes followed by an infusion of $0.5 \mu \mathrm{g} / \mathrm{kg} / \mathrm{hr}$ ) and a prospective assessment of the response to therapy using Finnegan scores.

RESULTS: The study cohort included 7 infants ranging in age from 3 to 24 months and in weight from 4.6 to $15.4 \mathrm{kgs}$. The patients were breathing spontaneously, having tolerated tracheal extubation 24-48 hours prior to starting dexmedetomidine. The patients had received a continuous fentanyl infusion with intermittent doses of midazolam for 4 to 7 days during mechanical ventilation. The fentanyl infusion was gradually decreased in 3 patients and discontinued without weaning in 4 patients. All infants manifested signs and symptoms of severe withdrawal with a Finnegan score $>12$. Withdrawal was effectively controlled by dexmedetomidine in the 7 patients. Subsequent Finnegan scores were $<7$ at all times (median 4, range 1 to 7 ). Two patients required a repeat of the loading dose and an increase of the infusion to $0.7 \mu \mathrm{g} / \mathrm{kg} / \mathrm{hr}$. These 2 patients had received higher doses of fentanyl than the other 5 patients $(8.5 \pm 0.7$ versus $4.6 \pm 0.5 \mu \mathrm{g} / \mathrm{kg} / \mathrm{hr}, \mathrm{P}<0.0005)$. No adverse hemodynamic or respiratory effects related to dexmedetomidine were noted. Dexmedetomidine was weaned by $0.1 \mu \mathrm{g} / \mathrm{kg} / \mathrm{hr}$ every $12-24$ hours.

CONCLUSIONS: Dexmedetomidine effectively controls withdrawal behaviour in infants following prolonged opioid and benzodiazepine sedation.

\section{D13}

\section{LOCAL ANALGESIA WITHIN 1 TO 3 MINUTES FOR PEDIATRIC VENIPUNCTURE AND PERIPHERAL VENOUS CANNULATION PROCEDURES USING THE NEEDLE-FREE SYSTEM ALGRX 3268}

W Zempsky ${ }^{1}$, J Bean-Lijewski² ${ }^{2}$ R Kauffman ${ }^{3}$, J Koh ${ }^{4}$, S Malviya ${ }^{5}$, J Rose ${ }^{6}$, P Richards ${ }^{7}$

${ }^{1}$ Connecticut Children's Medical Center, Hartford, Connecticut; ${ }^{2}$ Scott \& White Memorial Hospital, Temple, Texas; ${ }^{3}$ Children's Mercy Hospitals \& Clinics, Kansas City, Missouri; ${ }^{4}$ Oregon Health \& Science University, Portland, Oregon; ${ }^{5}$ University of Michigan Health System, Ann Arbor, Michigan; ${ }^{6}$ Children's Hospital of Philadelphia, Philadephia, Pennsylvania; ${ }^{7}$ Corgentech Inc, Secaucus, New Jersey, USA

INTRODUCTION: Despite recent trends showing improvements in pain management among children, peripheral needlestick procedures remain a common source of pain and anxiety in this population.

OBJECTIVE: To determine whether ALGRX 3268, a sterile, prefilled, disposable, needle-free system that delivers lidocaine powder into the epidermis, produces effective local analgesia within 1-3 minutes for venipuncture and peripheral venous cannulation procedures in children. METHODS: This was a prospective, single-dose, multicenter, doubleblind, placebo-controlled study. Pediatric patients (3-18 years) were randomly assigned to receive ALGRX 3268 (0.5 mg lidocaine/20 bar pressure $[n=289])$ or placebo $(n=285)$ from an identically configured system at the antecubital fossa or back of the hand. Comfort of the needle-free administration was reported by the patient immediately after ALGRX 3268 actuation using the Wong-Baker FACES pain-rating scale (0-5). One to 3 minutes later, the resulting pain of venipuncture or peripheral venous cannulation procedures was self-reported using the Wong-Baker FACES scale. Safety was assessed by monitoring adverse events (AEs) and by screening the administration site for erythema, edema, pruritus, and petechiae.

RESULTS: Demographic characteristics, proportion of venipuncture and cannulation procedures, and delivery sites were similarly distributed in both treatment groups. Immediately following actuation, the mean FACES scores were 0.54 and 0.24 in the ALGRX 3268 and placebo groups, respectively. After venipuncture and cannulation, adjusted mean FACES scores were 1.73 and 2.08 in the ALGRX 3268 and placebo groups, respectively $(\mathrm{P}=0.007)$. All treatment-related AEs were mild and resolved without sequelae. These included mild erythema and edema. 
Petechiae were more frequent in the ALGRX 3268 group and were generally mild.

CONCLUSIONS: The needle-free system ALGRX 3268 was well tolerated and produced significant analgesia within 1-3 minutes in children undergoing venipuncture and peripheral venous cannulation procedures.

\section{D14}

HOME ORAL MORPHINE FOR PAIN FRACTURES IN CHILDREN WITH OSTEOGENESIS IMPERFECTA

B Tourniaire $^{1}$, V Forin ${ }^{2}$, D Annequin ${ }^{3}$

${ }^{1}$ Unité Douleur Hopital Trousseau; ${ }^{2}$ Rehabilitation orthopedic unit Hopital Trousseau; ${ }^{3}$ Unité Douleur, Centre de la migraine de I'enfant, Paris, France

INTRODUCTION: Children with osteogenesis imperfecta are at high risk of multiple fractures. Pain in fracture is severe but remains undertreated. Although predictable, the fractures remain "unexpected" and analgesia is not currently the first goal. Pain management could be improved by training parents and children to assess pain and rapidly administer an oral dose of morphine if necessary.

PATIENTS AND METHOD: We have developed a training program for home use of oral morphine. Explanations on pain assessment and treatment are given to children and parents. In case of fracture associated with severe pain, our written prescription is to give as soon as possible an oral dose of immediate release morphine: $0.5 \mathrm{mg} / \mathrm{kg}$ (up to $20 \mathrm{mg}$ ). We have assessed the efficacy and safety of our protocol.

RESULTS: 31 families received the "pain management training" and had oral morphine available at home and/or school. Till December 2005, the protocol was used in eleven children. Analgesia was very good for 4 of them, good for 2 (femur fractures) and it was insufficient in 4 cases ( 3 femurs, 1 open tibia/fibula). No side effects were observed. Fifteen children did not use morphine: 7 because no fracture occurred, 3 because the family forgot it, 3 because they feared an addiction or a bad interaction with anesthetic agents if they need rapid orthopedic surgery. One physician told the parents not to administer it and one pharmacist refused to deliver it!

CONCLUSION: Rapid administration of oral morphine by parents improved pain relief in 7 out of 11 children. In 4 cases analgesia was insufficient and the children should have received a higher dose of morphine. Fear of morphine abuse is present not only in some families but also among health professionals.

\section{D15}

A RANDOMIZED TRIAL OF READING PREOPERATIVELY TO DAY SURGERY CHILDREN TO REDUCE ANXIETY AND PAIN C Coates $^{1}$, P McGrath $^{1}$, C McDougall ${ }^{2}$, R Goldbloom ${ }^{1}$

'Dalhousie University; ${ }^{2}$ IWK Health Centre, Halifax, Nova Scotia

BACKGROUND: Children undergoing day surgery often experience anxiety. The use of reading age-appropriate books by volunteers in pediatric clinics has been implemented in many hospitals in an effort to distract and increase literacy skills in young children. Reading helps young children focus and relax. Using reading as an anxiolytic agent for children and parents during the preoperative stage of surgery or as an analgesic after surgery has not been examined. We examined the effect of reading age appropriate books to children and parents in the waiting area of a pediatric day surgery unit to determine if their anxiety levels could be reduced.

PARTICIPANTS AND METHODS: 200 children, aged 2-8 years old and their parents awaiting day surgery participated in a cluster randomized trial comparing usual care to a reading condition. Children's anxiety levels were measured twice, before and after being read to via Kuttner's face scale while parental anxiety levels were measured via Spielberger's State-Trait Anxiety Inventory (STAI). Parents were also asked to keep a diary for 48 hours during their child's recovery to record their child's level of anxiety and pain via face scales.

RESULTS: Anxiety levels of children aged 5-8 years old in the reading group were significantly reduced after reading in comparison to children in the control group. In contrast, children aged 2-4 years old in the reading group did not show a significant reduction in anxiety levels after being read to. The reading intervention was found to have no significant effects on parental anxiety levels and did not have an effect on the average anxiety or pain level of the children during recovery in comparison to the control group. The intervention was accepted by the children and appreciated by parents.

CONCLUSION: Reading to 5-8 year-old children immediately prior to day surgery results in a significant, measurable decrease in anxiety levels.

\section{D16}

HOW DO PEDIATRICIANS MANAGE IMMUNIZATION

PAIN?

L Potash ${ }^{1}$, A Taddio 2 , L Manley ${ }^{1,3}$, M Ipp ${ }^{4}$, V Shah ${ }^{4}$, M Sgro ${ }^{5}$,

${ }^{1}$ Department of Population Health Sciences, ${ }^{2}$ The Hospital for Sick Children; ${ }^{3}$ The University of Toronto; ${ }^{4}$ Department of Pediatrics, Mount Sinai Hospital, ${ }^{5}$ Department of Pediatrics, St Michael's Hospital, Toronto, Ontario

BACKGROUND: Routine immunizations are the most common source of iatrogenic pain in healthy children. Previous studies have demonstrated that needle poke pain is effectively managed with topical anesthetic agents whereas postimmunization pain is best managed with oral analgesics. To date, the frequency of utilization of specific interventions in clinical practice has not been investigated.

OBJECTIVE: To assess pediatricians' practices regarding analgesic use to prevent needle poke pain and postinjection pain during routine childhood immunization.

DESIGN/METHODS: Self-administered mailed survey of pediatricians in the Toronto area (Ontario) who administer immunizations to their patients.

RESULTS: $70 \%$ (138/197) of eligible pediatricians responded; $68.9 \%$ were male and the median number of years of practice was 18.5 (25th and 75th percentile; 10,27). The reported number of immunizations administered per week was 50 (28.5,75). Analgesics were reported to be 'rarely' or 'never' used to relieve pain during the needle poke in $57.9 \%$ of respondents. When analgesics were used to prevent pain during the needle poke, $80.2 \%$ and $45.5 \%$ of the respondents used acetaminophen and ibuprofen, respectively. Lidocaine-prilocaine and tetracaine were reportedly used by $12.1 \%$ and $2 \%$ of respondents, respectively. Nonpharmacological methods of reducing pain during the needle poke were reported by $96 \%$ of pediatricians, and included: breastfeeding/bottlefeeding; holding the infant; using distraction, pacifier, or a sweetening agent; and altering the injection technique. Postimmunization pain was managed with either acetaminophen or ibuprofen by $88.8 \%$ of respondents.

CONCLUSIONS: The use of analgesics for postimmunization pain has been well integrated into clinical practice by pediatricians. In contrast, topical anesthetics remain underutilized for managing pain during the needle poke. Nonpharmacological interventions during needle poke are utilized by almost all pediatricians. Based on these results, knowledge translation strategies designed to increase the utilization of topical anesthesia for injection pain are recommended.

\section{D17}

USE OF GABAPENTIN, CLONIDINE AND METHADONE AS ADJUVANT ANALGESICS IN 4 CHILDREN (AGED 1, 2, 3 AND 11) WITH SEVERE BURN INJURIES (40-85\%)

\section{$\underline{G \text { Bricher, }} \underline{C \text { Onishko, } B \text { Cassidy }}$}

Women's and Children's Hospital, Adelaide, Australia

This poster presents the experience of a pediatric acute pain service comprising a specialist nurse, a pediatric anesthetist and a pharmacist in providing care for burn injury patients. The four cases described occurred within a 6 month period, were unusual in the extensive nature of the burns and challenged our service. Pain service involvement extended over several months of each child's initial admission.

Opioid tolerance developed quickly and required the addition of adjuvant agents to achieve adequate analgesia. The use of gabapentin, clonidine and methadone in the management of pediatric acute burn injury was a learning process for us. The poster will present the clinical indications, doses used and the pharmacological effects of these adjuvant medications 
as well as the complications encountered and clinical outcomes. Additional challenges arose from the transcultural environment which influenced each of these cases. We would welcome a discussion.

\begin{tabular}{lllll}
$\begin{array}{l}\text { Child } \\
\text { Burn type } \\
\text { \& area }\end{array}$ & $\begin{array}{l}\text { Primary } \\
\text { analgesic agents }\end{array}$ & Problems & $\begin{array}{l}\text { Adjuvant } \\
\text { medications } \\
\text { used }\end{array}$ \\
\hline B (age 11) Flame $50 \%$ & Morphine, Ketamine & $\begin{array}{l}\text { Ketamine } \\
\text { hallucinations }\end{array}$ & $\begin{array}{l}\text { Methadone, } \\
\text { Clonidine }\end{array}$ \\
T (age 1) & Scald $40 \%$ & Morphine, Ketamine & $\begin{array}{l}\text { Hyperalgaesia, } \\
\text { neuroexcitability }\end{array}$ & $\begin{array}{l}\text { Methadone, } \\
\text { Gabapentin, } \\
\text { Clonidine } \\
\text { Methadone, }\end{array}$ \\
H (age 3) & Flame $85 \%$ & Morphine, Ketamine & $\begin{array}{l}\text { Gabapentin } \\
\text { Methadone, } \\
\text { Gabapentin, } \\
\text { Clonidine }\end{array}$ \\
\hline S (age 2) Scald 50\% & Morphine, ketamine & \\
& & &
\end{tabular}

\section{D18}

\section{CAUDAL EPIDURAL - TRAMADOL AS AN ADJUVANT IN PEDIATRIC PATIENTS}

I Doctor, D Amrutia

Department of Anesthesia, BJMedical College and Civil Hospital, Ahmedabad, India

INTRODUCTION: "Kiddie Caudal" are very much popular technique of the intra- and postoperative analgesia in pediatric patients due to its high success rate, less complications and better outcome.

AIMS OF STUDY: To evaluate effect of Inj Tramadol HCL caudal epidural as an adjuvant to Inj Bupivacaine HCL on duration of analgesia in children

MATERIAL AND METHOD: Double-blind randomized and prospective study was under taken in total 100 children, varying from 6 months to 8 years, belonging to physical status ASA grade I-II, posted for below umbilicus surgery. All the patients were divided into two groups Group-I Control Group ( $\mathrm{n}=50)$ - received $0.5 \mathrm{~mL}$ of $25 \%$ Inj Bupivacaine $\mathrm{HCL}+$ normal saline while Group-II Study Group $(\mathrm{n}=50)$ received $0.5 \mathrm{~mL} / \mathrm{kg}$ of $0.25 \%$ Inj Bupivacaine HCL + Inj Tramadol $1 \mathrm{mg} / \mathrm{kg}$. All patients received light GA with mask ventilation. Intraoperative vital signs were monitored, oxygen saturation, comfort to the surgeons and requirement of inhalation anesthetic agent were recorded every $10 \mathrm{~min}$ utes till the end of operation. Postoperative additionally complications, sedation score and Pain scores were observed in the recovery room.

OBSERVATION AND RESULTS: We observed less pain scores and requirement of rescue analgesic in tramadol Group II as compare to Control Group-I. The analgesia lasted for about 8-10 hours. Hemodynamics were stable in both the groups intra- and postoperatively. Sedation score was same in both the groups in immediate postoperative period. There were no complications noted in any patients.

CONCLUSION: We conclude from our study results that it is safe to use Inj Tramadol $1 \mathrm{mg} / \mathrm{kg}$ as an adjuvant to the local anesthetic Inj Bupivacaine by caudal epidural route in children.

\section{Psychosocial Determinants and Context; Epidemiology}

\section{E1}

\section{DISHONESTY ABOUT HIV STATUS: RISK FACTOR FOR AIDS/HIV ACQUISITION IN DEVELOPING COUNTRIES}

\section{P Fayeni, O Olawuyi}

University College Hospital, Ibadan, Nigeria

ISSUE: It was revealed by this research work that most youth in third worlds are not honest about their HIV status and this has been one of the greatest harm on health issues among youth in most developing countries. However, this is an obvious risk factor for the wild spread of the disease among youth in the third worlds
METHOD: A laboratory HIV screening study was carried out on the 350 samples of young men and women, mean age, 25.3, selected through Simple Sample Random Technique. The subjects were interviewed through standardized research questionnaire on their HIV status and the 200 subjects claimed they were confirmed HIV-negative, before their specimens [Semen, Blood, High Vaginal Swabs] were screened for HIV by ELIZA and Western Blot Method in the Sexually Transmitted Diseases Research Laboratory, Department of Medical Microbiology, University College Hospital, Ibadan, Nigeria.

RESULT: All the 350 subjects claimed they were HIV-negative but 24 were found to be HIV-positive after the laboratory screening tests. 311 out of the total sample size, 350, have had unprotected sexual intercourse before, 29 have had sex but with preventive measures, and only 10 have not had sexual intercourse before. While, 21 out the 24 screened HIV-positive have unprotected sexual intercourse before.

CONCLUSION: From this finding, it was found the dishonesty of an individual's HIV status is one of the greatest harm and risk factor for the high increase of HIV infection/acquisition among youth in Sub-Saharan Africa and most developing countries.

\section{E2}

CHILDREN'S APPROACH - AVOIDANCE COPING DURING PAINFUL MEDICAL PROCEDURES: IS IT A DICHOTOMY?

C Lim ${ }^{1}$, L Cohen ${ }^{1}$, S Leahy ${ }^{2}$

${ }^{1}$ Georgia State University; ${ }^{2}$ Children's Healthcare of Atlanta,

Atlanta, Georgia, USA

INTRODUCTION: Approach-avoidance is considered one of the major dimensions of coping in the pediatric pain literature. However, findings are mixed about which coping style is more beneficial in regards to children's pain and anxiety during medical procedures. In addition, little is known about whether children demonstrate a coping style that may be mixed, which would consist of both "approach" and "avoidance" behaviours. The current study examines approach-avoidance coping in children receiving venipunctures, as well as differences in pain and anxiety between the coping styles.

METHOD: Participants included 88 children, 3 to 11 years of age, receiving venipuncture procedures at a children's hospital in the southeastern United States. Children's approach-avoidant coping style was assessed by self-report, parent report, and nurse report. Children's procedural pain and anxiety was assessed via self-report, parent report, and nurse report immediately following the venipuncture procedure.

RESULTS: Analyses revealed that $28.4 \%$ of children identified themselves as "approachers", $36.4 \%$ identified themselves as "avoiders", and $35.2 \%$ identified themselves as having a "mixed" coping style during the venipuncture. Results revealed significant differences in children's pain during the venipuncture according to parent report. There were significant differences between approachers and avoiders, with parents reporting children who identified themselves as avoiders exhibiting more pain compared to children who identified themselves as approachers. There were no differences between the mixed copers and the approach and avoidance copers in parent report of child pain. The three coping styles did not differ in terms of self-report of pain, parent report of child anxiety, and nurse report of child pain or anxiety.

DISCUSSION: In general, more than one-third of children identified themselves as having a mixed coping style. Children who identified themselves as approachers generally reported and were rated by parents and nurses as experiencing less pain and anxiety during a painful medical procedure than children who identified themselves as avoiders. Although further research is necessary, these results highlight the importance of considering children's coping styles along a continuum as opposed to a dichotomy. 


\section{E3}

\section{PERCEIVED SOCIAL SUPPORT, PSYCHOLOGICAL ADJUSTMENT, AND FUNCTIONAL ABILITY IN YOUTHS WITH PHYSICAL DISABILITIES}

$\underline{\text { S Wilson, L Washington, LEngel, M Jensen }}$

University of Washington, Seattle, Washington, USA

The aim of the present study was to examine the relationships between perceived social support, psychological adjustment, and functional ability in youths with physical disabilities, while also examining the potentially moderating effects of age and gross motor functioning on these relationships. Participants included a convenience sample of 70 youths (mean age $=14.51$ years; $60 \%$ male; $81 \%$ Caucasian; $58 \%$ ambulatory) from a large metropolitan area, with a primary diagnosis of neuromuscular disease $(n=37)$ or spina bifida $(n=33)$. Data were collected through semistructured youth interviews and parent questionnaires, and included demographic- and disability-related items, including a measure of gross motor functioning, and measures assessing psychological adjustment (Child Health Questionnaire Child Form 87 Mental Health Scale), functional ability (Functional Disability Inventory), and perceived social support (Multidimensional Scale of Social Support). Regression analyses indicated that higher levels of perceived social support from family, but not from friends, were significantly associated with better psychological adjustment. Furthermore, significant interactions emerged between family support and age, as well as between friend support and gross motor functioning, in the prediction of functional ability. Specifically, higher perceived family support was associated with higher functional ability among older youths, but with lower functional ability among younger youths. Higher perceived friend support was associated with higher functional ability among youths with more severe ambulatory restrictions, but not among youths with more mild ambulatory restrictions. The findings indicate that perceived social support plays an important role in psychological adjustment and functional ability in this population, and that the nature of this role may be moderated, to some extent, by age and level of gross motor functioning.

\section{E4}

IMPAIRED QUALITY OF LIFE AMONG PEDIATRIC PATIENTS REFERRED TO A SPECIALTY PAIN CENTRE

A Sato $^{1}$, W Davies ${ }^{1,2}$, K Khan ${ }^{2,3}$, R Ladwig' ${ }^{2}, K$ Hainsworth ${ }^{2}$, A Ferrise $^{2}$, A Hains ${ }^{1}$, S Weisman ${ }^{2,3}$

${ }^{1}$ University of Wisconsin; ${ }^{2}$ Children's Hospital of Wisconsin; ${ }^{3}$ Medical College of Wisconsin, Milwaukee, Wisconsin, USA

Chronic pain in children and adolescents results in increased school absences, sleep disturbances, and decreased social opportunities with peers. However, despite the relatively high prevalence of chronic pain among youth, quality of life (QOL) has not been well examined in the pediatric pain populations. Therefore, the goal of this study was to examine QOL in a clinical sample of youth with chronic pain.

The Pediatric Quality of Life Inventory, Version 4.0 (Varni et al, 2001; PedsQL 4.0) Generic Core Scales was administered to 92 patients with chronic pain unresponsive to conventional treatment $(65.6 \%$ female, mean age $13.4,86.0 \%$ Caucasian) presenting to a multidisciplinary pediatric pain clinic and 91 of their mothers. The PedsQL Generic Core Scales (Physical, Emotional, Social, School) are child self-report and parent proxy report scales developed to measure QOL among children and adolescents ages 2-18 years old. Most (55.6\%) patients had experienced pain for over one year (pain intensity $=5.8 / 10$ ). The PedsQL 4.0 child report total score (Cronbach's alpha $=0.89$ ) was lower for the entire group $(\mathrm{M}=58.1, \mathrm{SD}=18.8)$ compared with healthy norms $(\mathrm{M}=83.0, \mathrm{SD}=14.8)$ (Varni et al, 2001). Similarly, the PedsQL 4.0 mother proxy report total score (Cronbach's alpha $=0.90)$ was lower for the entire group $(M=54.8$, $\mathrm{SD}=18.5)$ compared with ratings by parents of healthy children $(\mathrm{M}=87.6$, $\mathrm{SD}=12.3)$. Positive correlations $(\mathrm{P}<0.01)$ were observed between each of the respective child report and mother report PedsQL 4.0 total scores and generic core scale scores $(\mathrm{r}=0.68-0.86)$.

Results suggest that youth presenting clinically with chronic pain experience significant disruptions in quality of life, as rated by both mother and child.

\section{E5}

\section{SOCIAL CONSEQUENCES OF PAIN: PARENT-CHILD AGREEMENT AND ASSOCIATIONS WITH CHILD AGE AND GENDER}

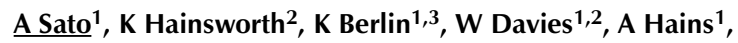
R Ladwig', K Khan ${ }^{2,3}$, A Ferrise ${ }^{3}$, S Weisman ${ }^{2,3}$

${ }^{1}$ University of Wisconsin; ${ }^{2}$ Children's Hospital of Wisconsin;

${ }^{3}$ Medical College of Wisconsin, Milwaukee, Wisconsin, USA

Social consequences of pain play a central role in conceptual models of the development and maintenance of pain-related behaviours among children. There is a paucity of conflicting literature comparing parent and youth perceptions of social consequences in pediatric chronic pain. Previous research has also failed to reach a consensus regarding the nature of relationships between child age, gender, and social consequences of pain. Therefore, the current study seeks to examine agreement between pediatric pain patients' reports of their pain-related social interactions and their parents' reports of these interactions, and to investigate the possible influences of youth gender and age on both youth and parent perceptions. Participants were 98 youth with chronic pain $(57.5 \%$ female, mean age 13.36) presenting to a multidisciplinary pain clinic and 94 of their mothers. Youth and their mothers completed the 32-item Social Consequences of Pain questionnaire (SCP; Walker et al, 2002), which measures social consequences of pain in interaction with parents, teachers, and peers to youth ages 8 to 18 . Consequences are measured across four domains - Positive Attention, Activity Restriction, Negative Attention, and Privileges.

Intercorrelations between SCP mother report and youth report subscales revealed significant correlations $(\mathrm{P}<0.01)$ between all four respective mother report and youth report subscales (range $r=0.36-0.81$ ). Multivariate analysis of variance indicated a moderate effect of child gender on youth report Positive Attention $(\mathrm{P}<0.05, \mathrm{~F}=5.63, \mathrm{~d}=-0.52)$, such that females reported more positive attention when in pain. Pearson correlations indicated a significant $(\mathrm{P}<0.01)$ correlation of -0.35 between youth age and youth report Positive Attention, such that younger children reported more positive attention when in pain. Results suggest youth and their mothers share similar perceptions regarding social consequences of pediatric chronic pain, and that youth age and gender may affect youths' perceptions of positive attention.

\section{E6}

PAIN IN CHILDREN AND ADOLESCENTS LIVING WITH CYSTIC FIBROSIS: A SURVEY OF MARITIME CANADIANS $\underline{\text { S Stenekes }}^{1}$, A Hughes ${ }^{1,2}, M$ Grégoire ${ }^{1,2}$, G Frager ${ }^{1,2}$

${ }^{1}$ IWK Health Centre; ${ }^{2}$ Dalhousie University, Halifax, Nova Scotia

Pain is a symptom known to be experienced by individuals living with cystic fibrosis. However, the prevalence and intensity of pain has rarely been studied in this patient population, especially the pediatric population. With the increased longevity of individuals living with cystic fibrosis, appropriate symptom management is necessary for quality of life. METHOD: This retrospective mail-out survey, sent to individuals living with cystic fibrosis, evaluated pain, dyspnea and cough and included a chart review. The study had a 55\% response rate, with 107 respondents ranging in age from 7-60 years. More than half of the respondents were children, ages $7-17$ years $(n=59,55 \%)$. The respondents are reasonably healthy individuals, with $65 \%(n=69)$ having no hospital admissions in the last year. Data collection occurred from June-December 2005 in 3 Canadian provinces: New Brunswick, Nova Scotia, and Prince Edward Island.

RESULTS: Pain intensity was rated using the Numerical Rating Scale (NRS) and further information was obtained regarding the location, duration, and treatments employed to manage pain. Pain was reported by $84 \%$ of participants. The most common locations of pain reported included: headache $(n=56,52 \%$, mean NRS 5.0), abdominal pain $(n=52,49 \%$, mean 5.4), trunk/back pain ( $\mathrm{n}=18,17 \%$, mean NRS 7.0$)$ and chest/lung pain $(n=18,17 \%$, mean NRS 4.2). Various treatments were utilized by this patient population and will be described in detail. This poster will compare the pain locations, intensity, and treatments utilized by the children and adult participants. This comparison may provide insight into symptom 
progression and burden of disease experienced as individuals with cystic fibrosis progress from pediatric to adolescent and adult care.

CONCLUSION: Even in healthy individuals living with cystic fibrosis, pain contributes significantly to the overall burden of disease. Consistent assessment is necessary to address the pain these individuals experience.

\section{E7}

\section{INTERPRETING PAIN EXPERIENCES: WHAT PAIN MEANS TO YOUNG BALLET DANCERS}

\section{F Visram $^{1}$, C von Baeyer ${ }^{1}$, D Eakins ${ }^{2}$, A Kant ${ }^{3}$, J Gold ${ }^{3,4}$}

${ }^{1}$ University of Saskatchewan, Saskatoon, Saskatchewan; ${ }^{2}$ Dalhousie University, Halifax, Nova Scotia; ${ }^{3}$ Children's Hospital Los Angeles; ${ }^{4}$ USC Keck School of Medicine, Los Angeles, California, USA

How do young ballet dancers perceive and interpret their pain experiences and how do these meanings of pain influence their responses to pain and injury? Research has looked at the impact of personality traits and coping styles on pain among adult ballet dancers, but there has been little research on the experiences of pain among young ballet dancers. In this multisite qualitative study we use interpretative phenomenological analysis to explore dancers' understanding of pain. Ballet dancers aged 12 to 16 years are being interviewed who are either preparing for the transition to pointe work (rising to the tips of the toes while performing) or who have been dancing en pointe for at least one year. A panel comprised of a former ballet dancer and experts in pediatric pain analyze each transcript from the semi-structured interview for themes and note connections between them. The themes are subsequently translated into a narrative account for each participant. Preliminary emergent themes from two interviews conducted with pre-pointe participants include: "pain indicative of working correctly," "pain indicative of harm," "interaction of mood and pain," "control over pain," "hiding pain," and "ability to cope with pain defines identity as dancer." Dancers indicated that the meanings they create about pain are influenced by peers, family members, and dance instructors. Themes and narrative accounts from participants will be interpreted in light of the current literature on pain experiences in sports, social expressions of pain, and specifically the world of ballet and competitive dance. Understanding the influence of pain perception and interpretation on pain management and prevention is important in identifying appropriate intervention strategies to minimize pain associated with ballet.

\section{E8}

\section{PARENTAL CHRONIC PAIN, PARENTAL COPING BEHAVIOUR, AND ACUTE PROCEDURAL PAIN AND DISTRESS IN 14-MONTH-OLD CHILDREN}

N Wolff, J Hunfeld, A Darlington, N van den Berg, S Kuiters, A Hofman, V Jaddoe, F Verhulst, J Passchier, H Tiemeier Erasmus Medical Centre, Rotterdam, The Netherlands

When a child undergoes a painful medical procedure, the child's pain and distress levels are influenced by parental behaviour. This behaviour is, among other things, dependent on the parent's own experiences with acute pain and possibly on the parent's experiences with chronic pain. While previous studies have investigated the relationship between the behaviour of healthy parents and child pain and distress during a medical procedure, no research exists on parents with chronic pain and their interactions with their young children in this situation. The present investigation examines the behaviours of parents with chronic pain and their 14-month-old children. This study is embedded in the focus-cohort $(n= \pm 1,200)$ of the Generation R study, a prospective cohort study $(n= \pm 10,000)$ from early fetal life into adulthood, carried out in Rotterdam, the Netherlands. Child pain, child distress, and parental behaviour during the painful procedure (venipuncture) are now being videotaped and scored. Parents will be divided into two groups, based on whether they reported chronic pain (defined as an illness that causes chronic pain) or not.

Initial data on parental chronic pain from the Generation R study indicate that $31 \%$ of mothers and $19 \%$ of fathers have one or more illnesses causing chronic pain. In $7 \%$ of children, both parents have one or more illnesses causing chronic pain.

We hypothesize that (1) children show more pain and distress from venipuncture when their parent displays more distress promoting behaviours (eg, apologizing) and that they show less pain and distress when their parent displays more coping promoting behaviours (eg, distraction), (2) parents who suffer from chronic pain show more distress promoting behaviours than parents without chronic pain, subsequently influencing the child's pain and distress levels. Preliminary results will be presented and discussed.

\section{E9}

\section{ANXIETY SENSITIVITY, FEAR OF PAIN AND PAIN-RELATED DISABILITY IN CHILDREN AND ADOLESCENTS WITH CHRONIC PAIN}

A Martin 1,2, P McGrath 2,3, S Brown 2,3, T Hart ${ }^{1}$, J Katz ${ }^{1,3}$

${ }^{1}$ Psychology, York University; ${ }^{2}$ Anaesthesia, The Hospital for Sick Children; ${ }^{3}$ Anesthesia, The University of Toronto, Toronto, Ontario BACKGROUND: Chronic pain is a serious problem for many children, but the risk factors for developing a chronic pain condition remain largely unknown. Anxiety sensitivity, a fear of bodily sensations typically associated with anxiety, appears to be an important vulnerability factor for the development of chronic pain and pain disability in adults. Adults who are highly anxiety sensitive fear and avoid their pain problems more than those who have low anxiety sensitivity. Converging lines of evidence support a fear-avoidance model in several adult chronic pain conditions. However, no study has examined these factors in children with chronic pain.

OBJECTIVE: To examine the relationships among anxiety sensitivity, fear of pain and pain-related disability in children and adolescents with chronic pain.

METHODS: An interview and 3 questionnaires (Childhood Anxiety Sensitivity Index, Pain-Anxiety Symptoms Scale, Functional Disability Inventory) were administered to 21 children and adolescents 8-17 years of age $(\mathrm{M}$ age $=14.2, \mathrm{SD}=2.2)$, who continued to experience pain an average of 4 years after discharge from the Chronic Pain Clinic at the Hospital for Sick Children.

RESULTS: Linear regression analyses revealed that anxiety sensitivity accounted for $38.6 \%$ of the variance in fear of pain $(F(1,20)=11.3$, $\mathrm{P}=0.003)$, fear of pain accounted for $39.9 \%$ of the variance in pain-related disability $(\mathrm{F}(1,20)=11.95, \mathrm{P}=0.003)$, but anxiety sensitivity was not significantly related to pain disability $(\mathrm{R} 2=0.09, \mathrm{P}>0.05)$.

CONCLUSION: These findings indicate that children with high levels of anxiety sensitivity had a higher fear of pain, which in turn was linked to increased pain disability. These results are consistent with the adult chronic pain literature and suggest that anxiety sensitivity and fear of pain may play important, and distinct, roles in the processes that maintain chronic pain and pain-related disability for children.

\section{E10}

\section{PREVALENCE OF PAIN IN PATIENTS WHO HAVE EXPERIENCED AN OBSTETRIC BRACHIAL PLEXUS INJURY G Olsson, C Stromback \\ Karolinska Institute, Stockholm, Sweden}

Chronic pain has hitherto not been thought to be a feature in patients that have experienced an obstetrical brachial plexus injury (OBPI). However, at the Pain Treatment Service at Astrid Lindgren Children hospital in Stockholm, 2 patients with OBPI were referred during the last years with typical neuropathic pain. The Swedish Health and Welfare (SoS) bureau runs a birth registry were data, including complications, from all childbirths in Sweden is recorded. This registry started in 1974. Astrid Lindgren Children's hospital (ALB) constitutes a national centre for severe cases of OBPI. A registry at ALB was started in 1984. The aim of this study was to determine the prevalence of pain in patients with OBPP with the aid of the above mentioned registries.

MATERIALS AND METHODS: From the SoS registry a random sample of 600 cases, born between 1974 and 1984, with the diagnosis OBPI was retrieved (OBPI adult-group). A matched control group of 600 persons without this diagnosis was also retrieved (control-group). A third group consisted of 412 patients, born from 1984, that had visited the plexus unit at ALB (OBPI child-group).

To all groups a questionnaire on eg, the occurrence and characteristics of 
pain in a hand/arm/shoulder was sent.

RESULTS: Response rates were appr 50\%. In the OBPI adult group many cases had probably been mild and transient and only 50\% in this group knew that they had had the diagnosis. In the OBPI adult group 31\% had experienced pain in the hand/arm. In the OBPI child group $21 \%$ had had pain. In the control-group 7\% had had pain in an upper extremity. Pain character was often pricking, stabbing, lightning-like, and/or burning indicating a possible neuropathic type of pain.

CONCLUSION: Pain in the affected limb after OBPI is not uncommon, especially in the age group 20-30 years.

\section{E11}

\section{SELF-IDENTIFIED NEEDS OF YOUTH WITH COMPLEX} PAIN: A QUALITATIVE INQUIRY

\section{P Forgeron ${ }^{1,2}$, P McGrath ${ }^{1,2}$}

${ }^{1}$ IWK Health Centre; ${ }^{2}$ Dalhousie University, Halifax, Nova Scotia

PURPOSE: To explore the self-identified needs of adolescents living with chronic pain.

METHOD: Qualitative study using a focus group. Questions to guide the focus group included: "What does health mean?"; "What coping strategies have you found useful?"; "Do you believe that peer support would be helpful, and why?"; and "What would be the best way to prepare for transfer to adult care?" The health centre research ethics board approved this study. ELIGIBILITY: Nineteen youth, aged 12-18 years, who received care through the Chronic Pain Clinic at a tertiary care children's hospital were invited to participate. Six youth, age 13-17years, consented to participate. DATA COLLECTION \& ANALYSIS: The focus group was conducted by a clinical nurse specialist. The discussions were transcribed verbatim and thematic analysis was conducted. Participants were contacted by phone to verify the accuracy of themes.

RESULTS: Five themes emerged from the data; being normal, distraction is best, fitting in, school stress and readiness to transition to adult care. CONCLUSION: Youth attending a tertiary care pain clinic view themselves as different and unhealthy compared to their peers. Health is seen as "being normal"; "able to do whatever you want". Distraction was the primary strategy for coping with pain. Peers were seen as essential to wellbeing. Pain helped them discover their 'true friends'. Half of the participants felt that peers who had pain would provide a major source of support. Those not in favour of this support cited the idiosyncratic nature of pain. School was uniformly identified as the greatest source of stress. The thought of transition to adult care was overwhelming. They were unsure what skills they needed to transition, and asserted they were not ready to transition to adult services.

\section{E12}

\section{CHILDREN WITH LOW BACK PAIN: WHO WILL HAVE CHRONIC SYMPTOMS? RESULTS OF A FOUR-YEAR PROSPECTIVE COHORT STUDY}

\section{G lones ${ }^{1}$, A Silman ${ }^{2}$, G Macfarlane ${ }^{1}$}

${ }^{1}$ University of Aberdeen, Aberdeen; ${ }^{2}$ The University of Manchester, Manchester, United Kingdom

BACKGROUND: Recent studies have shown that low back pain (LBP) in children is common. Onset is predicted by mechanical (injury) and lifestyle factors, but particularly emotional and behavioural problems and the occurrence of other pre-existing somatic pain complaints. These factors have also been shown to be important predictors of LBP in adulthood. However, it is currently not known whether children with LBP are those most likely to experience chronic symptoms in adulthood.

Thus, the aim of this study was, among a cohort of children with LBP, to establish what proportion go on to have chronic symptoms, and to examine whether such children can be identified at an early stage.

METHODS: From a large questionnaire-based cross-sectional survey in 39 schools in the Northwest of England, 330 children aged 11-14 years, were identified as having current LBP. A number of potential risk factors for the persistence of symptoms were recorded, including: anthropometrical characteristics, daily mechanical load (school bag weight), lifestyle factors, psychosocial factors (emotional and behavioural problems), and aspects of LBP severity and history. Participants were then followed up by questionnaire one and four years later to identify those with persistent symptoms (LBP at both time points). Poisson regression was used to identify the predictors of persistent LBP. Results are presented as risk ratios (RR) with $95 \%$ confidence intervals $(95 \% \mathrm{CI})$.

RESULTS: 178 children provided data at both one- and four-year followup, of whom 46 (26\%) reported persistent LBP. There were no significant differences in risk of persistent LBP with age, or between boys and girls. A number of indicators of baseline LBP severity significantly predicted symptom persistence: long ( $>1 \mathrm{yr}$ ) duration of symptoms (RR: 2.2; 95\% CI: $1.2-4.1)$, high pain score $(>7 \mathrm{~cm}$ on a $10 \mathrm{~cm}$ visual analogue scale) $(1.8 ; 1.03-3.3)$, reporting pain all day $(1.9 ; 0.7-4.9)$, pain radiating to the leg $(2.2 ; 1.4-3.6)$, and disability $(2.7 ; 1.5-4.8)$. In addition, the strongest aetiological predictor of outcome was the report of peer relationship problems: compared to children with a low level of this behaviour, those in the middle and top tertile experienced a 2-fold and 3 -fold increase in the risk of persistent LBP $(1.9 ; 0.95-3.91$ and $2.7 ; 1.4-5.2$, respectively). CONCLUSIONS: This study has demonstrated that although reports of LBP are common among children, only a minority (approximately onequarter) will experience persistent pain. Clinical variables of severity were strong and consistent predictors of persistence. In addition, we have shown that psychosocial variables, which have been demonstrated as important predictors of LBP onset, are also important predictors of chronicity. Knowledge of such predictors of outcome are important if children with LBP seek medical care, to allow the targeting of management to those with highest likelihood of persistence.

\section{E13}

SYMPTOMS AND QUALITY OF LIFE IN DYING CHILDREN WITH CANCER - THE PARENT'S PERSPECTIVE

\section{B Zernikow $^{1}$, C Wamsler ${ }^{2}$, A Menke ${ }^{2}$, D Garske ${ }^{2}$, S Brun $^{2}$, W Henkel $^{2}$, S Friedrichsdorf ${ }^{2}$ \\ ${ }^{1}$ Children's Hospital Datteln - Witten/Herdecke University (IKP); ${ }^{2}$ IKP, Datteln, Germany}

OBJECTIVE: As part of the nationwide PATE project we investigated the present quality of palliative care for pediatric cancer patients from the family point of view .

METHODS: 50 families from 6 out of the 18 regional departments of pediatric oncology consented to cooperate. The parents were visited by an especially trained nurse or doctor to perform a semi-structured interview. RESULT: $76 \%$ of the children had died of malignancy progression, and $24 \%$ of treatment-related medical complications. $47 \%$ of the patients had died at home, while $53 \%$ had died in a clinic, a third of them on the intensive care unit. Main troublesome symptoms were pain $(86 \%)$, fatigue $(82 \%)$, loss of appetite or unintended weight loss $(71 \%)$, or dyspnea (63\%). Between $41 \%$ and $49 \%$ of the patients suffered anxiety, constipation, or emesis/vomiting. As perceived by the parents, treatment was most effective against pain and constipation. $79 \%$ of the parents judged the received quality of care as "very good" or "good", $21 \%$ as "satisfying" or "moderate", and none of them as "bad/unacceptable". The parents assigned special importance to good symptom control, to the opportunity for overt communication with the caregivers, and to being accepted by the caregivers as serious partners. Even more than 3 years after the death of their child many parents felt left alone with/in their grief/mourning. Relatives and friends still tried to avoid talking about the process of dying, or the death of their/the beloved one.

CONCLUSION: Troublesome physical and psychosocial symptoms are expressed by most of the dying children. Most of the parents expressed their gratefulness of having got the opportunity to communicate this experience to us.

Acknowledgments: PATE was initiated by the German Society of Paediatric Oncology and Haematology (GPOH). It was funded by the German Children's Cancer Fund (Deutsche Kinderkrebshilfe) and Mundipharma GmbH, Limburg, Germany. 


\section{E14}

\section{A TALE OF TWO COUNTRIES: A COMPARISON OF THE PERCEPTIONS OF ANALGESICS AMONG FINNISH AND AMERICAN PARENTS}

$\underline{\text { R Zisk }}^{1,3}$, M Grey ${ }^{1}$, Z Kain ${ }^{2}$, P Kankkunen ${ }^{4}$, A Pietilä ${ }^{4}$, H Kokki ${ }^{5,6}$, K Vehviläinen-Julkunen ${ }^{4,7}$

${ }^{1}$ Yale University School of Nursing; ${ }^{2}$ Departments of

Anesthesiology, Pediatrics, and Child Psychiatry; ${ }^{3}$ Department of Anesthesia, Yale University School of Medicine, New Haven,

Connecticut, USA; ${ }^{4}$ Department of Nursing Science; ${ }^{5}$ Department of Pharmacology and Toxicology, Kuopio, Finland; ${ }^{6}$ Department of Anesthesiology and Intensive Care; ${ }^{7}$ Research Unit, Kuopio University Hospital, Kuopio, Finland

PURPOSE: Parents are reluctant to provide their children with analgesia, even when pain is expected and experienced. Few studies have examined parental perceptions regarding the provision of analgesia and little is known regarding the effect of culture on pain perceptions. Thus, this study compared parental perceptions regarding children's analgesia in Finland and in the USA.

METHODS: Study participants consisted of 315 Finnish and 110 American parents of children undergoing surgery. Parents completed the Parental Perception of Children's Analgesia questionnaire (Kankkunen et al, 2003). Analysis of the data included descriptive statistics, cross tabulation and $\chi^{2}$.

RESULTS: The results demonstrated that: parents in both countries agreed on about $50 \%$ of the measure items. In both countries most parents agreed that analgesia should be given before the pain becomes severe, that analgesia doses at home should be similar to those provided in the hospital, and very few parents thought that use of analgesia in childhood may lead to use of drugs as adults. In contrast, American (29\%) more than Finnish parents (7\%) thought that analgesia should be given regularly $(\mathrm{P}<0.001)$ and before the pain becomes severe (American $81 \%$, Finnish 65\%, $\mathrm{P}=0.001$ ). However, American (59\%) more often than Finnish parents (38\%) stated that analgesia should be provided only if the reason for the pain is known $(\mathrm{P}=0.002)$, that children easily become dependent on analgesics (American $16 \%$, Finnish $10 \%, P=0.022$ ) and that analgesics for home use may be dangerous for children (American 21\%, Finnish 8\%, P<0.001).

CONCLUSIONS: The findings demonstrate that: parents from both cultures have some reservations about providing their children with analgesia, cultural differences exist between American and Finnish parents and additional studies are necessary to further examine the effect of culture on pain perceptions.

Kankkunen P, Vehvilainen-Julkunen K, Pietila AM, Kokki H,

Halonen P. Parents' perceptions and use of analgesics at home after children's day surgery. Paediatr Anaesth 2003;13:132-40.

\section{E15}

\section{ADOLESCENT AUTONOMY AND HEADACHE-RELATED PAIN AND DISABILITY}

\section{T Palermo, J Putnam, T Koch}

Oregon Health and Science University, Portland, Oregon, USA

Recurrent headaches are the most common pain problem experienced by adolescents. An area that has received limited scientific investigation is the impact of family factors on adolescents' experience of pain and disability. The aims of this study are to examine the relationships between a range of family factors, including autonomy and general family functioning, on headache outcomes (ie, pain and activity limitations) in a population of adolescents with chronic/recurrent headaches using survey and observational methodology. A sample of 44 adolescents, ages 11 to 16 years, ( mean age $=13.5$ years, $61.4 \%$ female) receiving treatment through pediatric neurology for recurrent headaches have been enrolled. Adolescents and their parents completed measures of pain, functional disability, depressive symptoms, and family functioning (ie, McMaster Family Assessment Device, FAD). Observational data were collected from 27 families in three video-taped parent-teen interactions. These interactions were coded using the parent and teen subscales for rating Age-Appropriate Independence and Responsibility-Taking from the Autonomy and Intimacy Rating
S INC, DONOT COPY

System. Correlational analyses demonstrate that behavioural autonomy as measured by survey self-report is significantly related to activity limitations $(\mathrm{r}=0.51)$, where decreased autonomy is associated with increased activity limitations. Families characterized as unhealthy on the FAD had significantly higher levels of adolescent reported pain intensity, activity limitations, and depressive symptoms as compared to families characterized as having healthy functioning. Analysis of the observational data demonstrated that greater adolescent autonomy is associated with lower reports of pain intensity $(\mathrm{r}=-0.56)$, fewer activity limitations $(\mathrm{r}=-0.39)$, and fewer depressive symptoms $(r=-0.44)$. Findings suggest that autonomy may be an important dimension of adolescent and family functioning to consider in family-based pain treatment programs. Future plans for this study include increasing study sample size, and coding the remaining video-taped interactions.

\section{E16}

\section{INDIVIDUAL AND SITUATIONAL PREDICTORS OF PAIN- SPECIFIC MEMORY BIAS}

\section{Marche, $C$ von Baeyer}

University of Saskatchewan, Saskatoon, Saskatchewan

Memory of painful experiences can influence future coping with pain. The completeness and consistency of children's memory of painful events depends on several individual difference and situational factors. However, little is known about the influence of pain-specific memory biases (ie, special focus on, and memory for, pain-related information) on children's memory for pain and whether the effects of such biases differ across individuals. We examined whether individual differences, especially the presence of chronic pain, and the delay from the event until recall influence children's memory accuracy and bias for an experimental pain stimulus. Twenty-three children without pain (ages 7-15) and their parents provided information on individual differences in age, gender, coping style, pain catastrophizing, self-efficacy, state and trait anxiety, depression, pain sensitivity, and parental encouragement of illness behaviour. After undergoing the Cold Pressor Task (CPT), an experimental pain stimulus which requires children to immerse one arm in circulating water $\left(6^{\circ} \mathrm{C}\right)$, accuracy of children's memory for different pain components (e.g., sensory, affective, behavioural, cognitive, procedural, contextual) was tracked across a two week delay. Preliminary data with the nonchronic pain sample indicate the existence of a pain-specific memory bias for some children: (1) younger children's (ages 7-10) memory accuracy was best for the affective component, (2) children with a more pain sensitive temperament remembered more procedural details than children who were less sensitive to pain, and (3) children who reported that they experienced more pain exhibited a memory bias for cognitive details. These pilot data indicate that certain children may be biased to remember and recall pain-related information. Data with the chronic pain sample are forthcoming. Memory biases may make future coping with pain difficult. Understanding children's memory for painful experiences will improve their treatment and pain management.

\section{E17}

\section{CHILDREN'S EDITING OF INACCURATE MEMORIES FOR AN INJECTION PROCEDURE}

\section{Marche, L Spagrud, K McEown}

University of Saskatchewan, Saskatoon, Saskatchewan

Some children with diabetes experience fear and anxiety when they undergo their multiple daily blood glucose testing and injection procedures. Distress and pain during clinical procedures are associated with exaggerated (inaccurate) memory for the details of the procedure. The goal of this study was to determine, with the use of a memory model that measures false memory editing, whether children who report more pain and distress for their diabetic injections and finger pokes experience more difficulty rejecting false reports about a particular blood glucose test and injection experience. Seventy-eight 8 - to 16 -year-olds who were attending a summer camp for children with diabetes participated. Participants rated their pain/distress with the Facial Affective Scale and the Colored Analogue Scale for a finger poke and injection and completed a modified 
version of the Spielberger State/Trait Anxiety Inventory for Children. Researchers observed participants during a morning finger poke and injection and, later that afternoon, participants were asked to recall their morning pain/distress and to rate expected pain/distress for their next poke and injection. They were asked to recall event details and were instructed to either report only details that actually happened, only details that could have happened but did not, or both. Performance was compared across these conditions to assess the ability to edit inaccurate information from memory. The level of distress and pain reported, as well as anticipated pain and anxiety, were low. Using a false memory editing model, distress and pain did not consistently influence memory editing. However, age, blood sugar, and length of time since diagnosis influenced editing of false memories. Low pain and anxiety are not associated with memory editing. Future research should examine children's memory for events that are more painful or frightening than those examined in this study.

\section{E18}

\section{THE DEVELOPMENT OF ADOLESCENT CHRONIC PAIN: TESTING A PSYCHOSOCIAL MODEL USING A POPULATION-LEVEL APPROACH}

E Stanford $^{1}$, E Chen ${ }^{1}$, C Chambers ${ }^{2}$

\section{${ }^{1}$ University of British Columbia, Vancouver, British Columbia;}

\section{${ }^{2}$ Dalhousie University, Halifax, Nova Scotia}

Recurrent pain is a complex type of chronic pain that is experienced in intense episodes and surges in prevalence during adolescence. Psychosocial models for the development of adolescent recurrent pain need to be developed and tested. We propose that there are underlying factors associated with developing pain, such as gender, pubertal status and having a parent with chronic pain. Precipitating factors, for example, injury, acute illness, and stressful life events are likely to interact with underlying factors to influence adolescent psychological factors, such as anxiety, depression, and low self-esteem. These psychological factors may then propel the development of recurrent pain in adolescents. The proposed diathesis-stress model of adolescent recurrent pain builds upon a model for adult chronic pain proposed by Turk (2002). The research aimed to test this model using a nationally representative cohort of 2,488 10 and 11-year-olds from Statistics Canada's National Longitudinal Survey of Children and Youth (NLSCY). Participants, studied 5 times over 10 years, were asked about their head, stomach and back pain. Models for head, stomach and back pain were tested independently using growth curve and structural equation modeling techniques. Head pain was the most common type of weekly pain, reported by 26\% of adolescents in Cycle 2, 32\% in Cycle 3, 29\% in Cycle 4, and 28\% in Cycle 5. (Cycle 2-5 range for stomach pain: $14-22 \%$; back pain: $18-26 \%)$. Preliminary regression models testing whether selfesteem and anxiety/depression at Cycle 1 predicted pain at Cycle 2 were all significant. High anxiety/depression was a unique predictor of high head pain (Wald $z=68.6, \mathrm{P}<0.001$ ) and high stomach pain (Wald $z=37.1$, $\mathrm{P}<0.001$ ). Low self-esteem (Wald $z=7.5, \mathrm{P}<0.01$ ) and high anxiety/depression (Wald $z=31.8, \mathrm{P}<0.001$ ) were unique predictors of high back pain. The empirically generated models should facilitate research on developing effective prevention and treatment programs for adolescent pain.

\section{E19}

\section{PROGNOSTIC FACTORS OF CHRONIC PAIN AND DISABILITY IN THE PEDIATRIC POPULATION: A STEP TOWARDS PREVENTIVE ACTIONS}

\section{J Miró, A Huguet, R Nieto, S Paredes, J Baos} Department of Psychology, Rovira i Virgili University, Tarragona, Spain

The aim of our work was to identify what factors seem to be related to chronic pain and disability in the pediatric population. The underlying assumption is that from a clear understanding of these factors effective preventive measures could be developed for pediatric chronic pain patients. Two different approaches were pursued so as to learn about such prognostic factors. One focused on learning about experts beliefs on the issue. A three-stage Delphi poll was conducted to reach a consensus on what factors are potentially associated to chronic pain and disability.
DPC."DONOT COPY

Participants had to (1) identify those factors that from their point of view played a role on the chronification of pain and/or the disability, and (2) what was the extent of such an influence. Second, a review of the literature for the period of January 1996 to December 2005, on factors associated with idiopathic chronic pain and/or disability in the childhood was carried out.

As expected, there are important differences between experts opinions and results from studies in the literature on what factors are the most important ones to predict chronic pain and disability. While some opinions coincide with what is available in the literature, others are controversial and not well-sustained by the empirical evidence, whereas some others need to be empirically studied to check for their predictive validity.

In spite of the methodological weakness of both approaches, they give us the opportunity to gain insights into the factors potentially related to chronic pain and disability in the pediatric population, so that we could try to fill in the gaps currently present in the literature to advance our understanding on this topic.

This research was supported in part by grant FISO2/1353 from the Fondo de Investigaciones Sanitarias, Instituto de Salud Carlos III, Spanish Ministry of Health, and by the Agència de Gestió d'Ajuts a la Recerca, Department of Universities and Research, Generalitat de Catalunya.

\section{E20}

\section{LONG-TERM BEHAVIOURAL EFFECTS OF NEONATAL PAIN EXPOSURE}

I de Graaf, R Schouw, D Tibboel, J Peters

Erasmus MC - Sophia Children's Hospital, Rotterdam, The

Netherlands

AIMS: To evaluate whether surgery or mechanical ventilation in early infancy alters psychosocial functioning in later life. Second to assess the role of parental stress and parental psychopathology on the development of these problems.

METHODS: A prospective case-control study was conducted. After undergoing major surgery $(n=58)$ or mechanical ventilation $(n=53)$ during early infancy, infants were followed for up to 6-11 years (mean age 8.2 years). At this age their parents and teachers completed the Child Behaviour Checklist (CBCL) and Teacher's Report Form (TRF), respectively. To assess the role of parental stress and parental psychopathology, parents also filled out the Parental Stress Index and the General Health Questionnaire. Findings were compared with age- and gender-matched controls.

RESULTS: Univariate logistic regression analysis showed that former patients had significantly more anxiety/depression problems on the CBCL and more internalizing and total problems on the TRF $(\mathrm{P}<0.05)$. However, after adding parental stress and parental psychopathology to the logistic regression analysis, the differences between former patients and the matched controls disappeared; anxiety/depression, internalizing, and total problems, on the other hand, were predicted by parental stress $(\mathrm{P}<0.01)$ but not by parental psychopathology. Parental stress also predicted thinking, attention, and aggression problems on the CBCL and internalization and total problems on both the CBCL and TRF $(\mathrm{P}<0.05)$. Univariate analysis showed that parents of former patients reported significantly greater parental stress $(\mathrm{P}<0.01)$, but not more parental psychopathology problems than parents of the matched controls. No differences were found between children with a history of surgery and those who had been mechanically ventilated.

CONCLUSION: These preliminary findings suggest that pain or stress during early infancy does not influence behavioural and emotional development of children. Rather, it seems that neonatal hospital admission presumably affects parental stress mechanisms and that increased parental stress induces children's emotional behavioural problems later in life. 
E21

\section{UNDERSTANDING SCHOOL AVOIDANCE AMONG PEDIATRIC CHRONIC PAIN PATIENTS: THE IMPORTANCE OF MEASURING PAIN IN CONTEXT}

K Hainsworth ${ }^{1}$, K Anderson Khan ${ }^{1,2}$, R Ladwig², A Sato ${ }^{3}$, A Hains ${ }^{3}$, A Ferrise $^{1}$, S Weisman ${ }^{1,2}$, W Davies ${ }^{2,3}$

${ }^{1}$ Medical College of Wisconsin; ${ }^{2}$ Children's Hospital of Wisconsin; ${ }^{3}$ University of Wisconsin, Milwaukee, Wisconsin, USA

School avoidance (SA) has been recognized as a problem for youth with chronic and recurrent pain. One potentially important (and understudied) factor is the effect of context on the pain experience (Zeltzer et al, 1992). The purpose of this study was to examine whether school avoidance is related to contextual measures of child pain, as reported by both parents and children.

Mothers $(n=107)$ and children (13-18 years, $n=64)$ presenting to a multidisciplinary pain clinic completed contextual measures of child discomfort while in pain. Each question was rated on a 0 to 10 frequency scale anchored at $0=$ "not at all" and $10=$ "the worst." Questions required both parents and children to rate how emotionally and physically uncomfortable their child is while in pain, how emotionally and physically uncomfortable while in pain at school, and how emotionally and physically uncomfortable while staying home from school due to pain. Compared with other clinic families, mothers of SA patients rated their children as more worried/anxious when in pain $(\mathrm{M}=6.9$ vs $5.6, \mathrm{t}(103)=2.23, \mathrm{P}<0.05)$, and as more emotionally $(\mathrm{M}=7.9$ vs $6.0, \mathrm{t}(98)=3.25, \mathrm{P}<0.01)$ and physically $(\mathrm{M}=8.6$ vs $7.6, \mathrm{t}(100)=2.60, \mathrm{P}<0.05)$ uncomfortable while in pain at school. However, no differences were found for the measures focused on discomfort while staying home from school. SA patients' self-reports were higher than other patients on emotional discomfort while in pain $(\mathrm{M}=6.6$ vs 3.8, $\mathrm{t}(58)=3.95, \mathrm{P}<0.001)$, feelings of being angry/defiant while in pain $(\mathrm{M}=5.5$ vs $3.8, \mathrm{t}(60)=2.31, \mathrm{P}<0.05)$, and on emotional discomfort while in pain at school $(M=7.2$ vs $4.6, t(53)=2.75)$. Youth were also asked how uncomfortable their parents are when their child is in pain. SA youth reported greater parent discomfort $(\mathrm{M}=6.0$ vs $4.0, \mathrm{t}(59)=2.33, \mathrm{P}<0.05)$. These data suggest that contextual measures of pain may be important in the assessment of patients presenting with school avoidance.

\section{E22}

\section{VALIDATION OF A QUESTIONNAIRE VERSION OF THE CHILD ACTIVITY LIMITATIONS INTERVIEW}

K Hainsworth ${ }^{1}$, W Davies ${ }^{2,3}$, K Anderson Khan ${ }^{1,3}$, R Ladwig3, A Ferrise ${ }^{1}$, A Hains ${ }^{2}$, A Sato ${ }^{2}$, A Silverman ${ }^{3}$, S Weisman ${ }^{1,3}$

${ }^{1}$ Medical College of Wisconsin; ${ }^{2}$ University of WisconsinMilwaukee; ${ }^{3}$ Children's Hospital of Wisconsin, Milwaukee, Wisconsin, USA

The Child Activity Limitations Interview (CALI; Palermo et al, 2004) was developed to measure the day-to-day restrictions on a child's activity due to pain. Compared to the commonly used Functional Disability Interview (FDI; Walker \& Greene, 1991), the authors of the CALI suggest that the new tool is more relevant to children and adolescents, and is more sensitive to pain and to changes in disability over time. The authors call for future studies to address validation of modified versions, such as a selfreport paper and pencil version.

The current study involved a comparison of associations with other measures for the CALI and a questionnaire version (CALQ). Previous work has demonstrated excellent agreement between CALQ and CALI scores. Forty-five children and adolescents (8-17 years, $M=13,73.3 \%$ Caucasian, $77.7 \%$ female) and 43 parents $(29-60$ years, mean $=44,92.7 \%$ Caucasian, $87.8 \%$ mothers) were recruited from a multidisciplinary pain clinic. Both the CALI $(\mathrm{r}=0.566, \mathrm{P}<0.01)$ and the CALQ $(\mathrm{r}=0.582, \mathrm{P}<0.01)$ were significantly related to child reports of pain. The child and parent reports for both the CALI and the CALQ were strongly and significantly related to FDI Scores, and Quality of Life Scores (PedsQL, v 4.0, Varni et al, 2001), including PedsQL Total scores, and PedsQL Psychosocial Summary and Physical Summary Scores. Consistent with the initial validation report on the CALI, increasing age was related to increasing child-reported activity limitations (CALI: $\mathrm{r}=0.440, \mathrm{P}<0.01$; CALQ: $\mathrm{r}=0.578, \mathrm{P}<0.001$ ).
DPA " DO NOT COPY

Adolescents (13-17 years) reported greater limitations (CALI: $M=18.3$; CALQ: $M=23.2$ ) than children (8-12 years; CALI: $M=11.3$; CALQ: $\mathrm{M}=11.5$ ) on both scales.

These preliminary data suggest that like the CALI, the CALQ provides valid assessments of functional impairment in chronic pain patients and their families. These data also suggest that the validity of the tool is maintained with the more efficient paper and pencil format.

\section{E23}

\section{CHARACTERISTICS OF A PEDIATRIC POPULATION REFERRED TO A FRENCH MIGRAINE CONSULTATION}

D Annequin ${ }^{1}$, B Tourniaire ${ }^{2}$, E Gatbois ${ }^{2}$, A Tonnelli²,

G Mazaltarine $^{2}$, A De Crouy ${ }^{2}$

${ }^{1}$ Unite Douleur. Centre de la migraine de l'enfant; ${ }^{2}$ Centre de la Migraine de l'enfant Hopital Trousseau, Paris, France

The first centre devoted to the child migraine was officially created in 2002 within the framework of the priorities of the French second national program for pain control.

OBJECTIVE: To specify the characteristics of a pediatric population referred to a migraine specialized consultation

METHOD: This prospective survey was realized through a computerized form filled during each initial consultation. Migraine diagnosis was made according to international Headache Society (IHS) criteria updated in 2004 (Cephalalgia 2004; 24 S 1)

RESULTS: 970 children consulted over 17 months (Jan 04 to May 05). Age: means $10.5 \pm 3.5$ years; $6.5 \%$ are under 6 years; $29 \%$ are over 12 years DIAGNOSTIC: Migraine with aura and tension type headache (TTH): 34\%. Migraine without aura and TTH: 19\%. Migraine with aura: $17 \%$. Migraine without aura: $15 \%$. Migraine with chronic daily headache (CDH): $8 \%$. Sole TTH: 4\%. Sole CDH 2\%. Visual aura: 48\%; sensitive aura (paresthesias hand, foot) $28 \%$. Auditory aura: $27 \%$. Aura occurs during the headache in $52 \%$ of the cases and before the headache in $35 \%$. Duration of attacks: less 1 hour: $8 \%$; 1 at 2 h: $20 \% ; 2$ to 6 h: $32 \%$; 6 to 24 h: $24 \%$; 24 to 72 h: $12 \%$; over 72 h: $4 \%$. Half of children missed $7 \pm 8$ days of school because of migraine. $28 \%$ of these children missed between 8 and 42 days

CONCLUSION: In this population, migraine is very often associated with tension type headache in at least $50 \%$ of the cases. Aura is observed frequently. Adequate training is needed for doctors to improve their skill to identify different features of primary headache in children

\section{E24 BIOPSYCHOSOCIAL CORRELATES OF PEDIATRIC NONCARDIAC CHEST PAIN}

$\underline{\text { R Blount }}^{1}$, J Gilleland ${ }^{1}$, M Benoit ${ }^{1}$, M McCormick ${ }^{1}$, K Devine $^{1}$, L Simons ${ }^{1}$, B Reed ${ }^{1}$, G Johnson ${ }^{2}$, R Campbell ${ }^{3}$, K Dooley ${ }^{4}$ ${ }^{1}$ University of Georgia; ${ }^{2}$ Emory University School of Medicine, Athens; ${ }^{3}$ Emory University School of Medicine, Atlanta; ${ }^{4}$ Emory University School of Medicine, Snellville, Georgia, USA

Chest pain occurs in approximately $10 \%$ of children and is a frequent reason for referral to pediatric cardiology clinics. The most common causes of pediatric chest pain are idiopathic, or noncardiac, in nature. Noticing, interpreting, and communicating physical sensations in a particular manner are essential components in the history of the children and their families who are referred to cardiology clinics. Once noticed, others respond in various ways, including seeking medical attention.

The goal of this investigation was to extend our understanding of how biopsychosocial factors influence noncardiac chest pain. Participants were 8 to 18 year old patients diagnosed as having noncardiac chest pain. They were recruited at a pediatric cardiology clinic after their medical work up and prior to receiving their diagnosis and feedback from the cardiologist. Children and their parents completed a questionnaire assessing the frequency, duration, severity, and precipitating factors of their chest pain. In addition, parents completed measures of parental psychological symptoms and reports of child behavioural adjustment and somatization symptoms. Pediatric patients reported on their anxious, somatic, and depressive symptoms, and anxiety sensitivity. 
Results indicated that the highest level of chest pain experienced by the child was positively associated with the child's report of their frequency of other somatic symptoms, anxiety, and internalizing symptoms, and inversely associated with their level of adaptive behaviour. Similar correlational patterns were found for average chest pain severity. The frequency of the children's chest pain was positively associated with the mothers' report of their own somatization symptoms, and inversely related to the children's adaptive behaviours.

Results suggest that various parent and child psychological factors are significantly associated with pediatric chest pain frequency and intensity. It is possible that psychosocial interventions may be a viable treatment modality for reducing chest pain symptoms in these children and adolescents.

\section{E25}

\section{THE EFFECT OF CAREGIVER PERCEPTIONS OF CHILD VULNERABILITY ON FUNCTIONING AND HEALTH CARE UTILIZATION IN CHILDREN WITH CHRONIC PAIN}

\section{Connelly, K Anthony, J Wootton, S Rittgers, C Edwards,}

\section{Schanberg}

Duke University Medical Center, Durham, North Carolina, USA

Increasing recent attention has been placed on the social context in which chronic pain occurs. Within pediatrics, burgeoning research has uncovered parent or caregiver characteristics that may adversely impact functional outcomes in children and adolescents with chronic pain (eg, Chambers, Craig, \& Bennett, 2002; Logan \& Scharff, 2005; Peterson \& Palermo, 2004). However, little is known about the process through which parents or caregivers may impact child pain. The present study sought to evaluate the following: (a) whether caregiver psychological adjustment impacts functioning in children with chronic pain by virtue of the caregiver perceiving more vulnerability in the child; and (b) whether caregiver perceptions of child vulnerability manifest in increased utilization of health care resources. Eighty-seven (87) pediatric outpatients evaluated in an interdisciplinary pediatric pain clinic and their caregivers provided data for this study. Children completed measures of pain and psychosocial functioning, and caregivers completed measures of psychological adjustment and perceptions of child vulnerability. Caregivers also provided information about the health care utilization of their children. Results of mediational analyses using a series of multiple regression models provided support for the hypothesis that increases in perceptions of child vulnerability intercede the relationship between caregiver psychological adjustment and the functioning of children with chronic pain. Subsequent hierarchical regression analyses suggested that caregiver perceptions of child vulnerability may manifest behaviourally through increased health care utilization (regardless of the child's pain frequency and intensity). Caregivers with poorer psychological adjustment may perceive their children with chronic pain as more vulnerable, hampering child functioning by heightened vigilance to signs of discomfort and thereby maintaining a focus on pain. These data suggest that educating adult caregivers about the nature of chronic pain and helping them empower the child with chronic pain to independently develop and apply effective pain coping skills may improve child functioning and reduce utilization of health care resources.

\section{E26}

\section{THE ROLE OF PSYCHOLOGICAL FACTORS IN THE ETIOLOGY AND PERSISTENCE OF PEDIATRIC NONCARDIAC CHEST PAIN}

J Gilleland ${ }^{1}$, R Blount ${ }^{1}$, M Benoit ${ }^{1}$, M McCormick ${ }^{1}$, K Devine', L Simons ${ }^{1}$, B Reed ${ }^{1}$, G Johnson ${ }^{2}$, R Campbell ${ }^{2}$, K Dooley ${ }^{3}$ ${ }^{1}$ University of Georgia; ${ }^{2}$ Emory University School of Medicine, Atlanta; ${ }^{3}$ Emory University School of Medicine, Snellville, Georgia, USA

Chest pain occurs in about 1 in 10 school-age children (Garber, Walker, \& Zeman, 1991). Among children with noncardiac chest pain, the results of an unstructured interview indicated that psychiatric symptoms were found in about $75 \%$ of the population, with anxiety being the most common symptom (Tunaogul et al, 1995). In addition, children with chest pain report more anxiety, physical symptoms of anxiety, tension, anxiety somatic symptoms, anxiety sensitivity, physiological arousal, and mental incapacitation than children with benign murmurs (Lipsitz et al, 2004). Although very little research has been done in the area of pediatric chest pain, the available data indicate that psychological factors play a prominent role in the etiology, severity, and maintenance of this pain condition.

The purpose of this study was to delineate factors peculiar to children and adolescents who present in a cardiac specialty clinic with noncardiac chest pain versus those who present with innocent murmurs. Patients were between the ages of 8 and 18 years. Data were collected after the medical work up and prior to meeting with the cardiologist to receive the results. Measures of children's somatic symptoms in addition to chest pain, functional impairment of daily activities, anxiety, and anxiety sensitivity were included.

Results indicate that children with noncardiac chest pain have significantly more somatic symptoms than children with innocent heart murmurs. Children with noncardiac chest pain also report significantly higher rates of functional disability than children with innocent murmurs. Finally, chest pain patients reported higher anxiety sensitivity and somatic and physiological symptoms of anxiety than the innocent murmur group. These results suggest key child behavioural and psychological factors to address in therapeutic programs designed to improve psychosocial functioning, decrease functional disability, and reduce somatic complaints.

\section{E27}

\section{REPRESENTATIONS OF PAIN IN AFRICAN CHILDREN LIVING IN AFRICA VERSUS LIVING IN THE SUBURBS OF A EUROPEAN CITY}

\section{A Fernandes, M Lomba, T Marti}

Coimbra College of Nursing, Coimbra, Portugal

The social and cultural background of children and their families plays an important role in children's pain.

This exploratory study pertained to identify the meanings that children give to pain and how they talk about it.

Two groups of primary school children (8- to 12-year-old) of African origin were approached and asked to "Write and draw what pain is for you". One group ( $\mathrm{n}=35)$ was composed by children living in Africa (Sao Tomé) and the other group ( $n=35)$ by children belonging to an immigrant community from an African country (Cabo Verde) living in a suburban neighbourhood of Lisboa, Portugal. Regarding the social and economic situation, more than half of the children in both groups, with no significant difference, were in the two lowest levels of a 5 point scale. The written compositions obtained were qualitatively analysed.

In both groups children write about pain relating it to the body (eg, pain in the knee) and/or to emotions (eg, pain hurts and I feel sad). Social events related to pain are described differently by the two groups in that immigrant children describe situations such as living in the street, being abandoned, gunshot or beated as sources of pain. Pain is described as a personal experience or an experience lived by others. It is generally referred to as "an enemy".

Children were able to conceptualize pain in the absence of a current pain event. Sensory, emotional and evaluative descriptors were used to write about pain. The events described in relation to pain were clearly related to the community and cultural context of the children and are likely to experience their pain experiences. 


\section{E28}

\section{OCCUPATIONAL VARIABLES AND MATERNAL JUDGMENTS OF INFANT PAIN}

$\underline{\text { S Ahola }}^{1}$, R Pillai RiddelI ${ }^{1}$, B Stevens ${ }^{2,3}$, L Din ${ }^{1}$, S Greenberg ${ }^{2,3}$

${ }^{1}$ York University; ${ }^{2}$ The Hospital for Sick Children; ${ }^{3}$ University of Toronto, Toronto, Ontario

Developmental psychology research denotes that maternal sensitivity to infant distress impacts infant behaviour. However, little research has examined factors that influence maternal perceptions of infant pain. The purpose of this study was to explore the relationship between occupational variables that may influence the amount of time spent with infant (maternal education, maternal work status, maternal hours of work, and whether mother was currently staying at home) and maternal ratings of their own infant's pain. Seventy-seven mothers $(M=33.01$ years old, $\mathrm{SD}=3.85$ years $)$ and infants $(\mathrm{M}=10.95$ months old, $\mathrm{SD}=4.77$ months; range 5 to 20 months) were recruited from a pediatrician's office during routine immunization procedures. Mothers were asked to provide pain ratings for their infant's immunization pain directly after the needle. Mothers utilized three different pain scales (numeric rating scale; modified version of the Gracely Descriptor Differential Scales [pain intensity and pain unpleasantness; Gracely \& Kwilosz, 1988]) to judge their child's pain. A meta-pain scale, approximately ranging from 0 to 10 , was created from the three pain scales used by the mothers. Three 1-way analyses of variance and one $t$ test were used to separately analyze pain ratings on the different occupational variables. Maternal pain ratings immediately following their child's immunization were not significantly influenced by their education, work status, hours of work or whether they were currently in the home. Overall, the maternal occupational variables did not significantly relate to their infant pain ratings. This suggests that maternal sensitivity to infant pain may not be associated with quantity of time spent with the infant but rather the quality of time spent with their infant.

\section{E29}

\section{EPIDEMIOLOGY OF PAINFUL PROCEDURES AND ANALGESIC THERAPY IN NEONATES IN NEONATAL INTENSIVE CARE UNITS (NICUS) OF A DEVELOPING COUNTRY, PERU}

R Carbajal ${ }^{1}$, J Mucha ${ }^{2}$, M Raffo ${ }^{3}$, J Arias ${ }^{4}$, V Bao ${ }^{5}$, M Rivadeneyra ${ }^{6}$, J Zegarra ${ }^{7}$, C Bazan ${ }^{8}$, P Thibault ${ }^{1}$, D Annequin ${ }^{1}$

${ }^{1}$ Hôpital d'enfants Armand Trousseau, Paris, France; ${ }^{2}$ Hospital Guillermo Almenara; ${ }^{3}$ Hospital Santa Rosa; ${ }^{4}$ Instituto Nacional Materno Perinatal de Lima; ${ }^{5}$ Hospital Arzobispo Loayza; ${ }^{6} \mathrm{Hospital}$ Dos de Mayo; ${ }^{7}$ Hospital Daniel Alcides Carrion; ${ }^{8} \mathrm{Hospital}$ Nacional Materno Infantil San Bartolomé, Lima, Peru

BACKGROUND: Studies in industrialized countries have shown that neonates are exposed to numerous painful procedures in NICUs and that analgesic management is not optimal. There are no published data on this topic in developing countries.

OBJECTIVE: To determine the incidence of painful procedures and actual analgesic practices in NICUs of the city of Lima.

METHODS: During the first 14 days of hospitalization, data were collected prospectively on invasive procedures (and analgesic use) performed in 278 neonates admitted to 14 NICUs.

RESULTS: Mean \pm SD gestational age, birth weight and CRIB score were $35.2 \pm 3.8 \mathrm{wks}, 2245 \pm 885 \mathrm{gm}$, and $2.4 \pm 3.2$, respectively. 218 (78\%) infants were discharged before the 14 th day of hospitalization. 8748 procedures were performed in all neonates. $14 \%$ of procedures required more than 1 attempt. The 10 most frequent procedures were, $\mathrm{n}(\%)$ : heel stick 1953 (22.3), venipuncture 1775 (20.3), tracheal suctioning 928 (10.6), nasogastric tube insertion 661 (7.6), adhesive removal 638 (7.3), nasal suctioning 573 (6.6), x-ray 501 (5.7), arterial puncture 420 (4.8), endotracheal intubation 116 (1.3), umbilical catheter 112 (1.3).

$8522(97.4 \%)$ and $8371(95.7 \%)$ procedures were performed, respectively, without any continuous analgesia or sedation and without any analgesia for the procedure. Pharmacological and nonpharmacological analgesic therapy was rare, even for very painful procedures (ie, only 2 out 10 infants who had a chest tube insertion had a central analgesic).

\section{UP ING, DO NOT COPY}

CONCLUSION: A quarter of infants had more than 40 painful procedures (for some babies these were $>100$ ). Pharmacological and nonpharmacological therapy was greatly underused. Clinicians in developing countries cannot ignore that implementing sensitive analgesic management guidelines in their units is an obligation
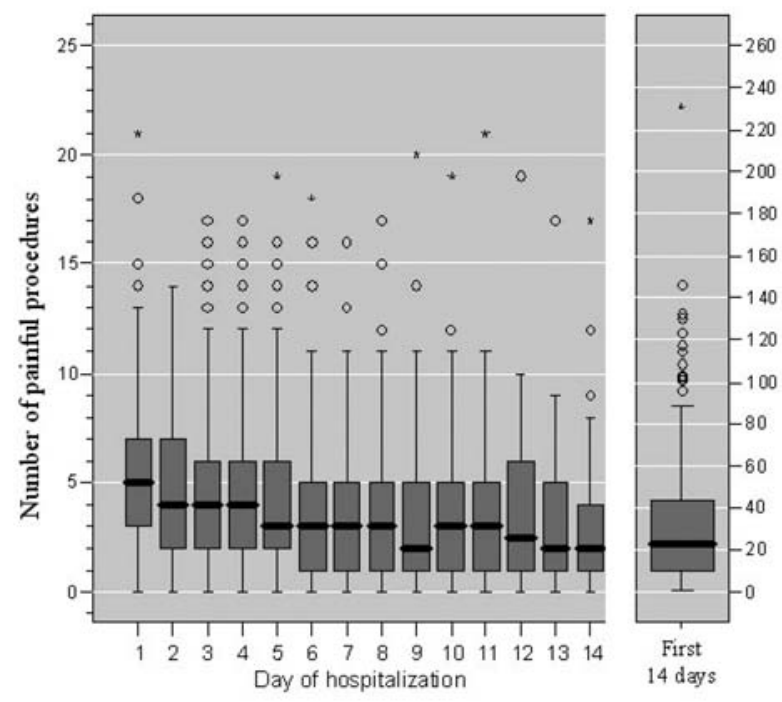

E30

PHYSICAL AND AFFECTIVE DISTRESS OF PAIN IN
ADOLESCENTS AND CHILDREN: MEASUREMENT
DEVELOPMENT AND INITIAL VALIDATION

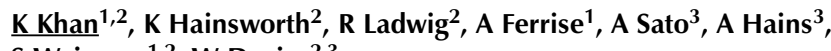
S Weisman ${ }^{1,2}$, W Davies, 2,3

${ }^{1}$ Medical College of Wisconsin; ${ }^{2}$ Children's Hospital of Wisconsin; ${ }^{3}$ University of Wisconsin, Milwaukee, Wisconsin, USA

Although many theoretical interpretations exist, "quintessentially, pain is experienced at a physical level and an affective level" (Fernandez \& Turk, 1992, p 205). In pediatric chronic pain, no efficient clinical measure exists to differentiate these dimensions of the pain experience. We present information on the development and validity of the measure: Physical and Affective Distress of Pain in Adolescents and Children (PADPAC).

This tool was developed for clinical assessment of parent/child perception of affective and physical distress related to chronic pain. Eight questions were designed to tap into 2 dimensions of child discomfort (affective/physical) while in pain, and in 2 contexts (home/school). Questions are rated from 1 = "not at all" to 10 = "the worst." Also rated are how "angry or defiant," and "worried or anxious" the child is while in pain. Children (6-17 years, $M=13.3, N=78)$, and their mothers $(\mathrm{N}=128)$ seen at a multidisciplinary pain clinic completed the scale at intake. MotherChild agreement was observed for all questions $(r=0.342-0.651, \mathrm{P}<0.05$ or better). Child-reported PADPAC ratings reflect the salience of the affective dimension of the youth's pain experience. Child-reported PedsQL (v. 4.0, Varni et al, 2001) Psychosocial Summary Scores and FDI (Walker \& Greene, 1991) scores were related primarily to ratings of emotional discomfort $(\mathrm{r}=-0.264$ to $-0.535, \mathrm{P}<0.05$ or better). PADPAC ratings were more distinct for the Mother proxy-report: questions addressing the physical dimension were related to PedsQL Physical Summary scores $(r=-0.204$ to $-0.371, \mathrm{P}<0.05$ or better $)$, whereas questions addressing emotional discomfort were related to the PedsQL Psychosocial Summary scores $(\mathrm{r}=-0.311$ to $-0.488, \mathrm{P}<0.01)$. Mothers' ratings of primarily physical discomfort while in pain were related to Motherreported FDI total scores $(r=-0.252$ to $-0.353, \mathrm{P}<0.01)$.

Initial results suggest that the PADPAC is a promising tool for understanding different aspects of pediatric chronic pain. 


\section{E31}

\section{HOW DO YOU COPE WITH PAIN? A PILOT STUDY IN A PEDIATRIC EMERGENCY DEPARTMENT IN CANADA}

\section{O Kristjansdottir, P McGrath}

\section{IWK Pediatric Pain Lab, Halifax, Nova Scotia}

The aim of this pilot study was twofold. Firstly to evaluate if the Charlestone Pediatric Pain Pictures (CPPP) (Belter et al, 1988) would prompt a discussion of pain coping in children across medical, play and home settings. Secondly to evaluate if the Pain Coping Questionnaire (PCQ) (Reid et al, 1998) can be used as a rating scale when analyzing children's interviews on pain coping experience.

The study was executed in a pediatric Emergency Department (ED) in Canada. The sample consisted of 26 children between the ages of 6 and 13 years old ( 8 female and 18 male) who required a venepuncture for a blood draw and/or IV start as part of their standard care in the ED. A semi structured interview was conducted with the children immediately after their venepuncture, asking them to talk about their coping experience related to the needle injection and day-to-day pain episodes (stomach ache, band aid removal, a cut on the knee, and a bee sting). Children were asked about what they did or would do in different pain contexts and what they thought about or would think about in these different pain contexts. The CPPP prompted a discussion of pain coping in children. Preliminary findings, using a grounded theory analysis, revealed a core variable "stop the hurt", which relates to two additional categories. These two descriptive categories were "internal coping strategies" and "external coping strategies", referring to whether the children found coping sources within themselves or from others when in pain. Further subcategories were "cognitive strategies" (eg, reassuring and destructive thinking) and "behavioural strategies" (eg, endurance).

These results indicate that two formerly excluded categories of the PCQ, palliative behaviours and indirect help seeking, need to be included when used as a rating scale for children coping with relatively short acute pain.

\section{E32 \\ DISTINGUISHING CLINICAL LEVELS OF DEPRESSION IN CHILDREN WITH CHRONIC PAIN SYNDROMES: INFLUENCE OF CAREGIVER DEPRESSION AND CHILD COPING STRATEGIES}

\section{Connelly}

Duke University Medical Center, Durham, North Carolina, USA

Depression is a commonly observed clinical feature of many children and adolescents with chronic pain, yet only recently have attempts been made at identifying characteristics that are predictive of depressed mood in these children (eg, Eccleston et al, 2004). We sought to determine the relative contribution of demographic data, pain characteristics, choice of pain coping strategies, and levels of caregiver depression to distinguishing children who were or were not clinically depressed based on Children's Depression Inventory (CDI) norms at the time of an initial pain evaluation. Eighty-seven (87) pediatric outpatients aged 7-18 evaluated in an interdisciplinary pediatric pain clinic for chronic pain complaints and their caregivers provided data for this study. Children completed measures of pain, depression (CDI), and use of coping strategies (KidCope), and the primary caregiver completed the Brief Symptom Inventory (from which the depression subscale was used for the present study). Results of discriminant function analyses conducted separately for younger children (ages 7-12) and adolescents (13-18) found that only the coping and caregiver depression variables significantly contributed to distinguishing clinical levels of depression in this sample. However, results varied by age: for younger children, caregiver depression was significantly more influential in distinguishing children who were versus who were not clinically depressed than child coping strategies. However, for the adolescents, avoidance of emotionally focused coping strategies and use of problemfocused strategies (eg, cognitive reframing) was a more important variable in predicting which adolescents were in the clinical range for depression than was caregiver depression. These data, albeit cross-sectional, suggest that interventions targeting depression in the pediatric pain population may need to be devised differently based on developmental variables: it may be more important to intervene at the caregiver level for younger children and at the individual patient level for adolescents.

\section{E33}

\section{THE RELATIONSHIP BETWEEN MATERNAL PSYCHOLOGICAL DISTRESS AND MATERNAL PAIN JUDGMENTS POSTIMMUNIZATION}

$\underline{\text { L Din }}^{1}$, R Pillai RiddelI ${ }^{1}$, B Stevens ${ }^{2}$, S Ahola ${ }^{1}$, S Greenberg ${ }^{2}$

${ }^{1}$ York University; ${ }^{2}$ University of Toronto, Toronto, Ontario

Craig's Sociocommunication Model of Infant Pain purports that infant pain is influenced by a caregiver's effectiveness in recognizing and managing infant pain. The goal of the present study was to explore the relationships between maternal ratings of infant pain, scores on a clinical screener of maternal psychological distress, and infant age. Seventy-seven mothers $(M=33.01$ years old, $S D=3.85$ years $)$ and their infants ( $M=10.95$ months old, $\mathrm{SD}=4.44$ months; range 5 to 20 months) were recruited from a pediatrician's office during a routine immunization procedure. Mothers were asked to judge their infant's pain for the period immediately following and the day after a routine immunization, using 3 different pain measures (Numeric Rating Scale [NRS]; Modified Intensity and Unpleasantness scales of the Descriptor Differential Scales [Gracely \& Kwilosz, 1988]). The 3 pain measures were equally weighted into a single scale representing maternal pain judgments (Pillai Riddell, 2004). Maternal psychopathology was measured using two global subscales of the Brief Symptom Index (BSI); Derogatis, 1993; the Global Severity Index (GSI), and the Positive Symptom Distress Index (PSDI). Maternal pain ratings, for each time point, were analyzed using a $2 \times 2$ between group analyses of variance (age $\times$ level of distress) for each global subscale of the BSI. A main effect was found for psychological distress level the day following the immunization on pain rating using the GSI but not when using the PSDI. There were significant main effects of infant age on pain rating at both time points. Mothers rated older infants as experiencing higher levels of pain on both days. These results demonstrate that maternal psychological distress does impact maternal pain judgments, and maternal pain judgments are systematically related to the age of the infant. Contributing factors including differential pain levels of various immunizations and maternal age-related infant biases will be discussed.

\section{E34}

\section{BELIEFS AND ATTITUDES OF CLINICIANS ABOUT PROCEDURAL PAIN IN NEONATES IN A DEVELOPING COUNTRY}

R Carbajal ${ }^{1}$, D Carbajal ${ }^{2}$, F Aburto ${ }^{3}$, J Gomez ${ }^{4}$, E Figueroa ${ }^{5}$, W Castillo ${ }^{6}$, I Linares ${ }^{7}, \mathrm{M} \mathrm{Gallo}^{8}$, P Cimerman 9 , D Annequin ${ }^{10}$ ${ }^{1}$ CNRD, Hôpital Trousseau, Assistance Publique Hôpitaux de Paris, Paris, France; ${ }^{2}$ Hospital Edgardo Rebagliatti Martins; ${ }^{3}$ Hospital Sabogal, Callao; ${ }^{4} \mathrm{Hospital}$ Naval; ${ }^{5} \mathrm{Hospital}$ de Policía; ${ }^{6} \mathrm{Hospital}$ Unanue; ${ }^{7}$ Hospital de la FAP; ${ }^{8}$ Hospital Militar, Lima, Peru; ${ }^{9}$ CNRD; ${ }^{10}$ Hôpital d'enfants Armand Trousseau, Paris, France OBJECTIVE: To know physicians' and nurses' estimates of neonatal procedural pain and their opinions about current and optimal neonatal procedural pain management in a developing country, Peru.

METHODS: A questionnaire was completed by 510 clinicians working in 14 level III neonatal units in Lima. They were asked to estimate the pain (scale 0-100) a neonate feels when he (she) undergoes 20 frequent procedures without analgesia. Opinions about the frequency of use of pharmacological and nonpharmacological treatments in their units were also asked as well as opinions about the optimal frequency of analgesic use for these procedures.

RESULTS: Respondents were $36.8 \%$ physicians, $42.8 \%$ nurses, $20.4 \%$ nurses auxiliaries; $78.2 \%$ female. Mean (SD) estimates of pain intensity were: chest tube insertion 83 (20), circumcision 78 (24), lumbar puncture 78 (21), peripheral arterial catheter 75 (22), tracheal intubation 74 (25), arterial puncture 74 (23), central venous line 74 (22), peripheral intravenous line 68 (24), venipuncture 66 (24), bladder catheter in males 66 (25), intramuscular injection 63 (26), bladder catheter in females 62 (26), heelstick 59 (26), subcutaneous injections 56 (25), umbilical line 
insertion 55 (28), tracheal suctioning 53 (27), nasogastric tube 48 (26), nasal suctioning 46 (26), eye examination 38 (27), and cranial ultrasound 20 (22). Clinicians believed that the frequency of use of pharmacological and nonpharmacological treatments for the 5 most painful procedures were, respectively: chest tube $(48 \%, 19 \%)$, circumcision $(45 \%, 24 \%)$, lumbar puncture $(14 \%, 28 \%)$, arterial catheter $(16 \%, 22 \%)$, and tracheal intubation $(28 \%, 16 \%)$. They thought that the optimal frequency of use of pharmacological treatment for these same procedures should be, respectively: $86 \%, 87 \%, 72 \%, 67 \%$, and $73 \%$

CONCLUSIONS: Though clinicians acknowledge that procedures are very painful in neonates, their current analgesic management is not optimal. There is an urgent need to surmount barriers responsible of this undertreatment of procedural pain.

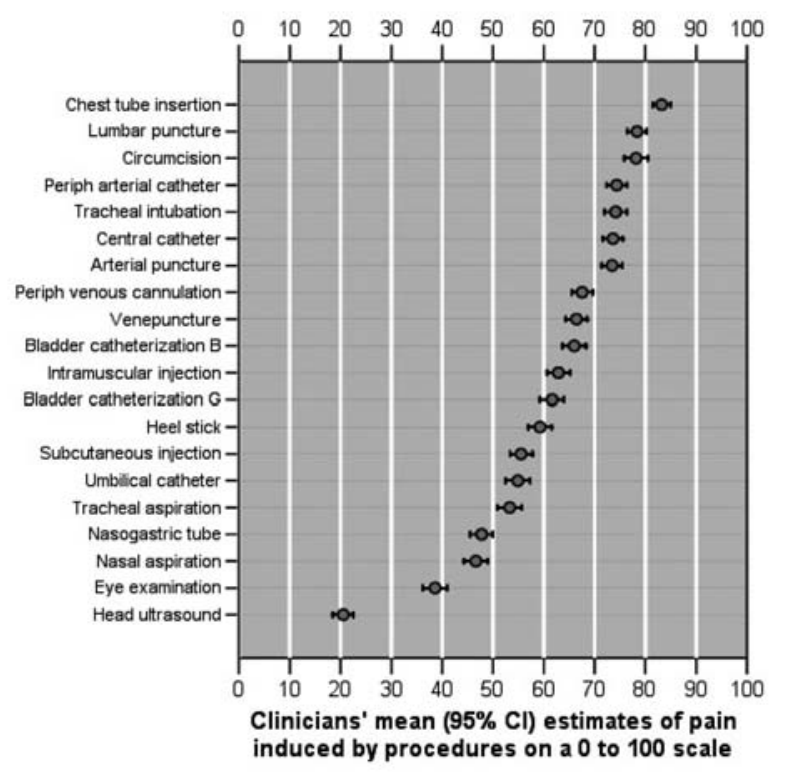

Acknowledgements: Funded by Fondation de France

\section{E35 \\ PROGRESS IN THE KNOWLEDGE OF PAIN IN LATIN AMERICAN PEDIATRICIANS}

$\underline{M}_{\text {Flores }}{ }^{1}$, V Adriana ${ }^{2}$

${ }^{1}$ IASP, Asociación Mexicana de Algología; ${ }^{2}$ Hospital Epidemiology Research Unity, Mexican Institute of Social Security (IMSS), México City, México

INTRODUCTION: Pediatric pain knowledge has increased in the last decade, unfortunately it is limited to a little medical population, and many children in pain around the world do not have medical attention.

OBJECTIVES: To compare the level of awareness of pain in Latin American pediatricians after eleven years.

METHODS: Cross-sectional study of 70 pediatricians. A survey was applied to a sample of 30 Mexican pediatricians in 1993 and to 40 Latin American pediatricians (Ecuador, Colombia, Brazil, Venezuela, Mexico, Dominican Republic, Argentina and Peru) in 2004. The subjects were asked to answer a questionnaire with 11 questions about different topics of pediatric pain. The data was analyze using the $\chi^{2}$ test (chi-square).

RESULTS: In 1993; 80\% of the subjects did not know of the existence of a method for pain assessment, $40 \%$ did not agree with the use of opioids in the management of chronic pain, $3.3 \%$ did know the existence of Trancutaneous Electric Neuro-Stimulation (TENS) and 13.3\% were informed about psychological methods for the treatment of pain. While in 2004, 42\% did not agree with the use of opioids, $7.5 \%$ knew TENS, $20 \%$ were informed about psychological methods for the treatment of pain.

CONCLUSIONS: The knowledge of the treatment of pain in children is limited even after eleven years has transpired. It is necessary to implement the teaching of pediatric algology in the residence of Pediatrics in Latin America.

\section{E36}

\section{PAIN REACTIVITY AND TEMPERAMENT PREDICT HEALTH BEHAVIOUR SEVEN YEARS LATER}

\section{E Rocha, K Prkachin}

Department of Psychology, University of Northern British

Columbia, Prince George, British Columbia

Population studies suggest that ill health can be determined by a host of psychosocial factors, including various forms of stress. However, the relationship between the experience of stressful events and health outcomes is empirically modest (Barr, Boyce \& Zeltzer, 1996). Barr et al (1996) have suggested that individual differences in psychobiological stress reactivity may account for the variability seen in illness or morbidity following stress, especially in children. Children's reactivity to one particular type of stress, namely pain, is the focus of this study. We sought to determine if children's pain reactivity to an inoculation and their temperament (both measured in kindergarten) are related to future health behaviour. Seven-year follow-up measures of somatization, health status, and health care utilization were gathered in forty-two children $(\mathrm{M}$ age $=11$ years $)$ who participated in our previous study of pain reactivity and somatization. Current health behaviour (assessed via parent and self-report measures) was compared with children's pain reactivity and temperament (measured in kindergarten). Results showed that children's pain reactivity at age five was related to children's self-reports of somatization seven years later $(\mathrm{P}=0.02, \mathrm{R} 2=0.13)$, independent of their temperament. Children scoring lower on the 'adjustment' factor of temperament (prone to negative mood and withdrawal, less adaptable) as rated at age five, had higher numbers of health care visits $(P<0.001, R 2=0.30)$, decreased psychosocial health $(P=0.04, \mathrm{R} 2=0.10)$, and increased selfreports of somatization $(\mathrm{P}=0.04, \mathrm{R} 2=0.10)$ when assessed seven years later. The present findings are among the first to suggest a prospective link between temperament and pain reactivity and health utilization. The data support the notion that early response styles (ie, heightened pain reactivity and difficult temperament) may indicate risk for increased health care utilization and poorer health and well-being later in childhood.

\section{Systematic Reviews}

\section{F1}

\section{COMPARISON OF EFFICACY OF BREAST-FEEDING WITH ORAL GLUCOSE ON INFANT VACCINATION PAIN}

\section{S Rostami, L Nikrooz, H Alijani, H Rasekh}

Ahvaz Jondishapour University of MedicalSciences Ahvaz Iran,

\section{Ahvaz, Iran}

The present clinical trail study was designed to compare the effect of breast-feeding with oral glucose on infant pain caused by vaccine shot. Samples were 194 healthy infants 2, 4, 6 months who referred to health care centre services of Yasog University of Medical Sciences. They randomly divided in three groups, 73 breast-feeding cases, 73 oral glucose cases, and 48 controls. In the breast-feeding group, breast-feeding was starting two minutes before injection DPT vaccine, and was continued till start of injection. In the oral glucose group, two minutes before injection DPT vaccine, $2 \mathrm{~mL}$ of $50 \%$ glucose was instilled on the baby's tongue, and in the control group, injection performed as routine (they did not have breast milk or oral glucose). In the three groups, procedure was videotaped. Film was recorded and stored in a computer. The modified behavioural pain scale (MBPS: minimum point: 0 , maximum point: 10) was used to assess pain scores during baseline 5 seconds before and 15 seconds after vaccination. The latency of the first and total crying time were measured. The findings show the MBPS scores were lower in the breast-feeding group (Mean=5.77) than oral glucose group (Mean=6.38). And the duration of crying in the 180 seconds was lower in the glucose group (50.36) than the breast-feeding group (62.27). Although entering data in T-test was determined, there is no significant difference between pain relief by breast-feeding and oral glucose. We suggest using breast-feeding before, during, and after vaccination procedure aimed with other interventions: baby touching and holding in mother's 
arm and lulling. That is pain relief during minor invasive procedures in infants.

\section{F2}

\section{DEVELOPMENTALLY SENSITIVE ADAPTATIONS TO THE COGNITIVE BEHAVIOUR THERAPY (CBT) APPROACH TO THE MANAGEMENT OF CHRONIC PAIN IN CHILDREN}

\section{R Khan}

Department of Psychological Medicine, The Children's Hospital at Westmead, Westmead, Australia

Cognitive Behaviour Therapy (CBT) is an individual, psychotherapeutic approach to the treatment of psychological disorders such as depression and anxiety. CBT has been shown to be effective in the treatment of psychological distress associated with chronic pain, in addition to improving pain tolerance and coping repertoire. A key component of CBT is the replacement of distorted thinking and learning more adaptive thoughts and behaviours. There is debate however, about whether the CBT approach is developmentally appropriate for young children experiencing chronic pain. This poster will explore methods of delivering CBT in a manner which is developmentally sensitive and engaging for children with chronic pain.

The multidisciplinary team at the Children's Hospital at Westmead Pain Clinic see approximately 100 outpatients a year with chronic pain of various etiologies. Psychological consultation is sought for over $50 \%$ of patients annually. In 2004-2005, psychological consultation was sought for children aged from 10-17years (mean 13.7 years). The developmentally sensitive, family-centred treatment approach developed for this cohort is constructed around four stages. Firstly, creative and fun strategies are used to engage the child and help them recognize psychological factors that may be associated with their increased pain levels. Secondly, therapists help children and their families make the difficult and abstract link between "emotional" pain and "physical pain", therefore forming the rationale for psychological treatment. Thirdly, children learn about the impact of an improved repertoire of coping strategies on the severity of pain. Finally, children are taught coping strategies which focus on learning to regulate their psychological distress and are tailored to their developmental and family contexts.

CBT is successfully used in the management of chronic pediatric pain when the pivotal concept of the relationship between emotions and physical pain are explored in an engaging and developmentally sensitive way. Understanding the child's pain and psychological response within the broader family-social environment is also an important extension of the individualized CBT approach in the management of pediatric pain.

\section{F3}

\section{PSYCHOLOGICAL SYMPTOMS, ADOLESCENT-PARENT RELATIONSHIPS, AND PARENTING STRESS IN THE CONTEXT OF ADOLESCENT CHRONIC PAIN}

LGuite $^{1,2}$, R McCue ${ }^{2}$, J Rose ${ }^{1,2,4}$, D Sherry,

${ }^{1}$ Pain Management Program, ${ }^{2}$ Department of Anesthesiology \& CCM, ${ }^{3}$ Division of Rheumatology, Department of Pediatrics, CHOP; ${ }^{4}$ University of Pennsylvania School of Medicine; Philadelphia, Pennsylvania, USA

This project examines relationships among adolescent and parent psychological symptoms, adolescent-parent relationships, and parenting stress as they relate to parental reinforcement of pain-related behaviour and adolescent functional disability. A retrospective record review was conducted for 112 adolescents with chronic pain (mean age $=15.88, \mathrm{SD}=1.27 ; 79 \%$ female) evaluated at a tertiary care pediatric pain management clinic and their parents $(88 \%$ mothers; mean age $=45.06, \mathrm{SD}=5.61)$. Information includes demographic data, pain history, the Brief Symptom Inventory, Stress Index for Parents of Adolescents, Illness Behaviour Encouragement Scale, and Functional Disability Inventory. We hypothesized that greater pain reinforcement and adolescent functional disability would be associated with greater: 1) adolescent and parent self-reported psychological distress; 2) parent-perceived adolescent intrapersonal distress and adolescentparent relationship distress; and 3) parenting stress. Results showed that adolescent- and parent-reported pain reinforcement $(r=0.57, \mathrm{P}<0.01)$ and disability $(\mathrm{r}=0.73, \mathrm{P}<0.01)$ were significantly correlated. Adolescent global psychological distress was associated with parent-reported pain reinforcement $(\mathrm{r}=0.30, \mathrm{p}<0.01)$, but not with adolescent-reported pain reinforcement. Adolescent somatic symptoms were associated with self$(\mathrm{r}=0.32, \mathrm{p}<0.01)$ and parent-reported disability $(\mathrm{r}=0.23, \mathrm{P}<0.05)$. Parent somatic $(r=0.20, P<0.05)$ and depressive symptoms $(r=0.20, P<0.05)$ were associated with parent-reported pain reinforcement. Less parent-perceived adolescent antisocial behaviour and adolescent-parent relationship distress was associated with greater adolescent-reported, but not parent-reported, pain reinforcement $(\mathrm{r}=-0.24, \mathrm{P}<0.05 ; \mathrm{r}=-0.39, \mathrm{P}<0.01$, respectively). Parent-perceived adolescent social isolation/withdrawal was correlated with greater parent-reported disability $(r=0.21, \mathrm{P}<0.05)$. Parent-perceived life restrictions related to parenting $(\mathrm{r}=0.29, \mathrm{P}<0.01)$ and parental feelings of social alienation $(r=0.20, P<0.05)$ were associated with parent-reported pain reinforcement. Results provide support for our hypotheses and highlight complex relationships among adolescent and parent factors that will be further considered. Study findings have implications for the development of family-focused intervention approaches to improve functional abilities among adolescents with chronic pain.

\section{F4}

\section{COMPARISON OF A SINGLE-DOSE EFFICACY OF $10 \mathrm{MG} / \mathrm{KG}$ IBUPROFEN OR $15 \mathrm{MG} /$ KG PARACETAMOL (ACETAMINOPHEN) AND MULTIPLE DOSE TOLERABILITY IN FEBRILE CHILDREN}

I Gibb $^{1}$, H Clarke ${ }^{1}$, H Shaw ${ }^{1}$, E Autret-Leca ${ }^{3}$, A Charlesworth ${ }^{2}$ ${ }^{1}$ Boots Healthcare International, ${ }^{2}$ Nottingham Clinical Research Limited, Nottingham, United Kingdom; ${ }^{3}$ University Hospital, Tours, France

AIM: To compare the efficacy of single dose $10 \mathrm{mg} / \mathrm{kg}$ ibuprofen to $15 \mathrm{mg} / \mathrm{kg}$ paracetamol and tolerability of up to 3 days' dosing in febrile children.

METHODS: A French multicenter, double-blind, parallel-group, prospective, randomized study of 300 children ( 3 months $-12 \mathrm{yrs}$ ), with tympanic temperature of $\geq 38.5^{\circ} \mathrm{C}$ to $\leq 40.5^{\circ} \mathrm{C}$ associated with various nonserious pathologies, were randomized to treatment. The primary end point was the area under the temperature reduction (absolute difference from baseline) curve from 0 to 6 hours. Multiple objective secondary temperature end points and tolerability of ibuprofen or paracetamol were also compared. Additionally, subjective parental opinion of treatment was recorded.

RESULTS: The treatment groups were well balanced at baseline for relevant variables such as temperature, age and weight. The results are summarized in the table below:

\begin{tabular}{|c|c|c|c|}
\hline End point & Ibuprofen & Paracetamol & $p$ value \\
\hline & $(n=151)$ & $(n=150)$ & \\
\hline AUC0-6 (Primary) & -7.77 & -7.66 & ns \\
\hline AUC0-4 & -4.88 & -4.96 & ns \\
\hline AUC0-8 & -10.13 & -9.07 & ns \\
\hline $\begin{array}{l}\text { Median time to maximal } \\
\text { temperature reduction }\end{array}$ & 240 mins & 240 mins & ns \\
\hline $\begin{array}{l}\% \text { parents rating drug as } \\
\text { very efficacious }\end{array}$ & 59.2 & 37.2 & $p<0.001^{\star \star \star *}$ \\
\hline $\begin{array}{l}\% \text { parents who would use } \\
\text { drug again }\end{array}$ & 96.5 & 88.8 & $p<0.05^{*}$ \\
\hline $\begin{array}{l}\text { Overall incidence of adverse } \\
\text { events (no. reports) }\end{array}$ & 21 & 21 & ns \\
\hline $\begin{array}{l}\text { Incidence of Gl events } \\
\text { (no. reports) }\end{array}$ & 4 & 4 & - \\
\hline
\end{tabular}

CONCLUSIONS: Both drugs were equally effective at reducing temperature. Tolerablity was comparable. Parental opinion of treatment and willingness to use again was superior for ibuprofen. This may be due to other effects that were not measured in this trial, for example analgesic effects. Ibuprofen may provide additional treatment benefits over paracetamol in febrile children. 
F5

\section{DO CHILDHOOD FACTORS PREDICT CHRONIC OR RECURRING PAIN: A SYSTEMATIC REVIEW OF COHORT STUDIES}

Lamont $^{1,2}$, T Piira ${ }^{2}$, G Champion ${ }^{2}$, M Lassere ${ }^{3,4}$

${ }^{1}$ Dalhousie Medical School, Halifax, Nova Scotia; ${ }^{2}$ Pain Research Unit, Sydney Children's Hospital, Randwick; ${ }^{3}$ St George Hospital, Faculty of Medicine; ${ }^{4}$ Faculty of Medicine, University of New South Wales, Sydney, Australia

BACKGROUND: A systematic review of the literature was conducted analyzing prospective studies evaluating the effect of childhood factors on the occurrence of subsequent chronic or recurrent pain. Areas where there is inadequate evidence, or the studies are of an insufficient quality will also be highlighted to help guide future research.

METHODS: A literature review was conducted searching Medline, PsychInfo, Embase, and CINAHL, as well as hand-searching. Inclusion critera was as follows: (i) prospective cohort studies; (ii) participants aged 18 years or less at time 1; (iii) the effects of childhood factors on subsequent pain outcomes were evaluated; (iv) follow-up data was obtained at least 4 months after initial data collection. Studies were excluded if the same data set was reported in multiple publications. Conclusions about relevance, quality, and results were tabulated by coding for characteristics of sample, sample size, study design, childhood factors assessed, outcome measures used, study quality, analyses conducted, and conclusions reached. RESULTS: The search terms yielded 4066 articles. A review of the abstracts identified 179 articles of potential relevance. Fifty-two articles met full inclusion criteria. Seven assessed more than one type of pain outcome, resulting in 62 data sets in total. Pain outcomes included: musculoskeletal pain, headache, abdominal pain, and other pains. Thirty childhood factors were assessed in at least two articles. Childhood factors that had the strongest evidence for predicting subsequent pain were: childhood pain, emotional/psychiatric problems, behavioural problems, and maternal and paternal health. Neither high BMI nor physical activity was found to predict subsequent pain.

CONCLUSIONS: Children with pain problems were more likely to also experience pain in later childhood and adulthood. A child's mood, personality and behaviour were also found to be predictive of pain. Physical health markers, such as level of physical activity and body mass index, were less important in predicting chronic/recurrent pain.

\section{F6}

\section{WHAT ARE WE SO AFRAID OF? PARENTAL ATTITUDES REGARDING CHILDREN'S ANALGESIA}

\section{R Zisk $^{1,2}$, M Grey $^{2}$, Z Kain ${ }^{3}$}

${ }^{1}$ Yale University School of Nursing; ${ }^{2}$ Department of Anesthesia;

${ }^{3}$ Departments of Anesthesiology, Pediatrics, and Child Psychiatry, Yale University School of Medicine, New Haven, Connecticut, USA PURPOSE: The reasons why parents undermedicate their children's pain at home, substitute less potent compounds for prescribed analgesia, and stretch dosing intervals are not well studied. Thus, this study examined parental perceptions regarding analgesia and explored the effects of socio-demographic, parental and child personality characteristics on these attitudes.

METHODS: Following consent, 110 parents of healthy children ages 3-14 undergoing elective surgery were recruited for this study. Attitudes and personality characteristics were examined using the Medication Attitude Questionnaire (MAQ), the EASI scale of child temperament, and the NEO-FFI five-factor inventory to examine parental personality. Analysis of the data included descriptive statistics, bivaraite statistics, factor analysis, and stepwise regression.

RESULTS: Parents stated that: "side effects are something to worry about when giving children analgesia" (73\%); "children should be given analgesia as little as possible because of side effects" (51\%); "analgesia is addictive" (43\%); "the less often children received analgesia the better it worked" (37\%); and "analgesia has many side effects" (30\%).

A factor analysis with Varimax rotation produced a 3-factor solution explaining $52 \%$ of the variance. The factors were application of use, side effects and avoidance. Application of use subscores were affected by child
DPC, DO NOT COPY

gender $(P=0.029)$, child impulsivity $(P=0.023)$, father ethnicity $(\mathrm{P}=0.047)$, and parental consciousness $(\mathrm{P}=0.003)$. Avoidance subscores were affected by parental education $(\mathrm{P}=0.001)$, child activity level $(\mathrm{P}<0.001)$, child sociability $(\mathrm{P}=0.005)$, and child age $(\mathrm{P}=0.047)$. Side effects subscores were affected by parental education $(\mathrm{P}<0.001)$, child impulsivity $(\mathrm{P}=0.022)$, and child agreeableness $(\mathrm{P}=0.02)$.

CONCLUSIONS: Parental higher education levels predicted more positive attitudes regarding analgesia safety. Child and parent personality characteristics also had an effect on parental attitudes regarding analgesia. Further studies are necessary to examine the effects of these variables on parental pain management practices.

\section{F7}

\section{CAN A HEATED PILLOW AND/OR BLOWING SOAP BUBBLES REDUCE ANXIETY, DISTRESS, AND PAIN AT NEEDLE PROCEDURES IN CHILDREN WITH CANCER?}

L Högberg ${ }^{1}$, L von Essen ${ }^{2}$, G Ljungman ${ }^{1}$

${ }^{1}$ Department of Women's and Children's Health; ${ }^{2}$ Department of Public Health and Caring Sciences, Uppsala University, Uppsala, Sweden

BACKGROUND: Children often mention procedures as the most painful, and distressing problem during illness, and treatment. The main aims were to examine whether children experience less anxiety, distress, and pain connected to a routine needle insertion in an intravenous port when subjected to 1) an intervention: heated pillow (HP) or blowing soap bubbles (BSB) in addition to standard care (SC) vs subjected to $\mathrm{SC}$, and when subjected to 2) SC+HP vs SC+BSB.

METHOD: Twenty-eight children 2-7 years, cared for at a pediatric oncology unit, undergoing a routine needle insertion in an intravenous port were included consecutively. All children were subjected to 2 needle insertions; at the first they received SC, and at the second SC, and a randomized intervention: HP or BSB. Parents and nurses assessed children's anxiety, distress, and pain on $0-100 \mathrm{~mm}$ visual analogue scales (VAS).

RESULTS: Parents reported less anxiety for children subjected to $\mathrm{SC}+\mathrm{HP} / \mathrm{SC}+\mathrm{BSB}$ vs SC $(\mathrm{n}=28)(\mathrm{F}=16.2, \mathrm{df}=1 / 26, \mathrm{P}=0.0005)$ and less anxiety $(\mathrm{F}=4.6, \mathrm{df}=1 / 26, \mathrm{P}=0.05)$, distress $(\mathrm{F}=9.2, \mathrm{df}=1 / 26, \mathrm{P}=0.01)$, and pain $(\mathrm{F}=6.6, \mathrm{df}=1 / 26, \mathrm{P}=0.05)$ for children subjected to $\mathrm{SC}+\mathrm{HP}(\mathrm{n}=14)$ vs $\mathrm{SC}+\mathrm{BSB}(\mathrm{n}=14)$.

Nurses' reports did not show any significant differences for $\mathrm{SC}+\mathrm{HP} / \mathrm{SC}+\mathrm{BSB}$ vs $\mathrm{SC}$, nor for $\mathrm{SC}+\mathrm{HP}$ vs $\mathrm{SC}+\mathrm{BSB}$.

CONCLUSIONS: According to parents, having a heated pillow or blowing soap bubbles is more effective than standard care in reducing children's anxiety in connection to needle procedures, and a heated pillow is more effective than blowing soap bubbles in reducing children's anxiety, distress, and pain.

\section{F8}

\section{MASTERING FEAR: TAKING THE PHOBIA OUT OF NEEDLE PHOBIA}

B Rosen

BC Children's Hospital, Vancouver, British Columbia

Despite advances in medical science, needles continue to be an often necessary pathway for administration of medicine in a variety of childhood illnesses - either for investigational procedures and/or treatment. Having a fear of needles can be extremely detrimental to children of all ages who require either ongoing blood-work to assess levels of medication in their blood, daily injections of medications for a specific period of time of throughout their life, or frequent investigations for diagnostic or treatment purposes. Needle phobia can interfere significantly with a child's health, illness treatment, and disrupt a child and family's coping with diagnoses and illness - sometimes more than the illness itself.

Many children and youth develop an incapacitating fear of needles after difficult or traumatic experiences involving injections or blood tests. These experiences can then create high anticipatory anxiety often culminating in absolute refusal to comply with the procedure or treatment. Too often, these fearful children are subsequently held down in an effort to accomplish the needle poke as quickly as possible with the mistaken belief 
that that is the only way to address the problem and a hope that "this will be the last poke". This approach unfortunately sets up a vicious circle of mistrust of the adults - caregivers and medical staff - greater fear of needles, as well as a more intense level of "noncompliance" for future situations.

This poster presentation will detail a step-by-step process of teaching children how to reduce the fear associated with needles and provide a level of mastery that has the added benefit of generalizing competent coping skills to other situations in the child's life. This is especially important when the child has to deal with an acute or chronic disease condition where treatment and evaluation involves frequent exposure to needles.

This program utilizes a combination of cognitive behaviour therapy, relaxation training, and graduated exposure.

\section{F9}

\section{THE EVALUATION OF FLACC AND COMFORT SCALES FOR PAIN ASSESSMENT IN INTUBATED AND VENTILATED CHILDREN AFTER SURGERY}

E Kokinsky, M Johansson

Department of Anaesthesia and Intensive Care, The Queen Silvia Children's Hospital, Sahlgrenska University Hospital, Göteborg, Sweden

BACKGROUND: The assessment of pain in intubated and ventilated children is a challenge. The COMFORT scale has been validated for measuring distress but has also been shown to be valid for pain assessment in PICU. The Face, Legs, Activity, Cry and Consolability (FLACC) pain scale has been validated for postoperative pain in spontaneously breathing children.

The aims of the study were to evaluate the validity and reliability of COMFORT and FLACC (slightly modified) scales for postoperative pain in intubated children in a critical care setting.

METHODS: One hundred-nineteen observations were recorded in 40 children. Two nurses familiar with both COMFORT and FLACC observed the patient during two minutes simultaneously, but without cooperation. Concurrently the bedside nurse and assisting nurse scored the pain by using a visual analogue scale (VAS) and scored the sedation on a fivegraded scale. Since FLACC has not been validated in intubated patients the ratings before and after analgesics were recorded in additional patients.

RESULTS: There were positive correlations between the FLACC and VAS and between the FLACC and sedation scores; gamma correlation coefficients were 0.51 and 0.46 respectively $(\mathrm{P}<0.05)$. The corresponding coefficients between the COMFORT and VAS and the COMFORT and sedation scores were 0.49 and $0.51(\mathrm{P}<0.05)$. A high correlation was found between COMFORT and FLACC, gamma correlation coefficient was $0.76(\mathrm{P}<0.05)$.

The interobserver reliability proved to be good; the weighted kappa value for COMFORT was 0.75 and for FLACC 0.65 .

After analgesic administration the FLACC median score decreased from 5 to 0 ( $\mathrm{P}<0.001$, Wilcoxon signed rank test).

CONCLUSION: The findings support the use of either the COMFORT or the FLACC scale to assess postoperative pain in intubated, ventilated children. The items in the scales seem to be indicators of both distress and pain.

\section{F10}

THE RELATIONSHIP BETWEEN PAIN, SLEEP, AND FATIGUE IN CHILDREN AND ADOLESCENTS WITH CANCER:

\section{A REVIEW}

V Gedaly-Duff ${ }^{1}$, L Nail ${ }^{1}$, A Johnson ${ }^{1}, K_{\text {Johnson }}{ }^{2}, \mathrm{~K} \mathrm{Lee}^{3}$,

${ }^{1}$ School of Nursing; ${ }^{2}$ School of Medicine, Oregon Health \& Science University, Portland, Oregon; ${ }^{3}$ University of California,

San Francisco School of Nursing, San Francisco, California, USA

BACKGROUND AND PURPOSE: The relationship between pain, sleep disturbance, and fatigue is complex, not well understood and under-researched in childhood cancer. While single symptom studies report that children and adolescents with cancer experience these symptoms, there is little literature that discusses the links between them.
This review summarizes the state of the literature on pain, sleep, and fatigue in children with cancer.

PAIN AND SLEEP: The experience of pain may be disease-related (eg, tumour), treatment-related (eg, chemotherapy; radiation), procedure/monitoring-related (eg, lumbar puncture, bone marrow aspiration), and/or normal development-related (eg, toothaches; menstruation). Pain may interfere with the quality and quantity of sleep such as an inability to get into deep sleep stages. Frequent arousals and awakenings cause sleep fragmentation leading to next day fatigue, sleepiness, and neurocognitive deficits. Threat-related awakenings to more pain may cause hyperarousal and insomnia. Simultaneously, an increased sensitivity to pain may occur because glucocorticoids, a cornerstone of chemotherapy, alter sleep structure and may lead to arousal and sleeplessness.

FATIGUE, PAIN AND SLEEP: In the few studies about fatigue in children with cancer, descriptions of pain and sleep disturbance were reported. Children differentiated typical tiredness from chemotherapyrelated fatigue. Although, anemia and metabolic changes may explain fatigue, other contributing factors to sleeping poorly at night may be anemia-related restless leg movements that disrupt sleep and anxiety. Children with cancer may have daytime sleepiness and nap. That in turn may lead to sleep problems at night because of an irregular sleep-wake cycle that further challenges their pain coping abilities.

CONCLUSION: Pain is commonly associated with other symptoms particularly sleep disturbance and fatigue. As children mature into adolescents, their pain expression and sleep patterns change. More research into the interactions between these symptoms, and their potentially synergistic effects within the context of maturation is needed.

\section{F11}

THE EVALUATION OF TWO SELF-REPORT PAIN SCALES IN A PAEDIATRIC EMERGENCY UNIT

$\underline{B}^{\text {Finnström }}{ }^{1,2}$, E Kokinsky ${ }^{1,3}$

${ }^{1}$ Department of Paediatric Anaesthesia and Intensive Care, The Queen Silvia Children's Hospital; ${ }^{2}$ Sahlgrenska University Hospital, Department of Nursing, Health and Culture, University West; ${ }^{3}$ Sahlgrenska University Hospital, Göteborg, Sweden BACKGROUND: The faces pain scales (FPS) are often used for assessment of pediatric pain although; they have been criticized because of confounding affective distress with pain intensity. The coloured analogue scale (CAS) is a modified visual analogue scale from 0 to 10 . The aims of the study were: to compare pain intensity ratings with two scales in "untrained" children, to evaluate construct validity by assessment of pain before and after analgesics, to evaluate children's preferences for the two scales.

METHODS: Sixty-two children in the emergency unit with pain were asked to mark their current pain on both self-report scales after informed consent. The FPS consisted of 6 faces also scored from 0 to 10 like the CAS. To assess construct validity, a group of children $(n=19)$ were asked to score their pain before and after administered analgesics. After the scorings the children were asked which scale they preferred.

RESULTS: For children 4.5-7 years $(n=29)$ the mean pain score was 5.2 (95\% confidence interval (CI): 4.4-6.0) on CAS and 5.2 (CI: 4.3-6.1) on FPS. For children $>8$ years $(n=33)$ the corresponding rating was 6.0 (CI: 5.6-6.8) on CAS and 5.9 (CI: 5.3-6.5) on FPS. The Spearman correlation coefficient between the scales was 0.64 for children $>8$ years and 0.67 for children $5-7$ years $(\mathrm{P}<0.05)$. Mean pain scores after analgesic administration decreased from 6.2 to 4.1 with CAS $(\mathrm{P}<0.01)$ and from 5.6 to 3.8 with FPS $(\mathrm{P}<0.01)$. More children $>8$ years preferred CAS (22/32) to FPS (10/32). Results indicated that younger children preferred FPS (17/28) to CAS (11/28).

CONCLUSION: Children's ratings on the FPS and the CAS agreed well. Both scales exhibit construct validity in untrained children in an emergency unit. Children $>8$ years preferred the CAS while younger children tended to prefer the FPS. 
F12

EPIDURAL ANALGESIA IN MAJOR NEONATAL SURGERY:

WORTHWHILE ALTERNATIVE?

I de Liefde ${ }^{1}$, M van Dijk1 ${ }^{1}$ D Tibboel ${ }^{1}$, A Bösenberg ${ }^{2}$

${ }^{1}$ Department of Pedriatric Surgery, Erasmus MC - Sophia

Children's Hospital, Rotterdam, The Netherlands; ${ }^{2}$ Department of

Anaesthesiology, Red Cross War Memorial Children's Hospital,

Cape Town, South Africa

BACKGROUND: Due to unwanted side effects of morphine such as respiratory depression resulting in mechanically ventilation, an alternative is sought for pain management in neonates after major surgery. Epidural anesthesia is well-established in adults, but not in children. In adults, epidurals provide better analgesia than intravenous morphine and results in a faster recovery and less morbidity (Rigg et al, 2002; Rodgers et al, 2000; Flisberg et al, 2003). However, it is unknown if neonates have similar advantages as adults. Therefore, we performed a retrospective study in which we compared epidural to nonepidural analgesia in neonates, who underwent surgery between 2002 and 2005 for thoracic (esophageal atresia) or abdominal (duodenum, jejunum or ileum atresia) surgery in the Red Cross War Memorial Children's Hospital, Cape Town, South Africa. RESULTS: Of 56 operated neonates, 23 received an epidural catheter with a bolus of bupivacaine $0.25 \%$ perioperatively and postoperatively continuous infusion with bupivacaine $0.2 \%$. Both groups were comparable for gender, gestational age and preoperative ventilation, but different for diagnosis, $65 \%$ thoracic surgery in epidural vs $22 \%$ in nonepidural group. Duration of anaesthesia was comparable. Postoperatively, twenty-four $(72 \%)$ neonates in the nonepidural group received intravenous morphine, as well as half of the neonates in the epidural group, often for sedative purposes. Although, total amount of morphine was comparable, the duration of morphine was significant longer in the epidural group (median $38 \mathrm{~h}$ (1-133) vs $20 \mathrm{~h}(5-109), \mathrm{P}=0.048)$. Duration of ventilation, length of stay on the ICU, number of reintubations and deaths were not significantly different between both groups. In 7 out of 23 neonates complications with epidurals consisted of blood or CSF take, blocked catheter and premature dislodgment of catheter out of the epidural space.

CONCLUSIONS: This research confirms the need for well-designed randomized trials to establish if epidural anaesthesia is suitable for neonates.

\section{F13}

\section{A REVIEW OF MORPHINE AND HYDROMORPHONE PCA SIDE EFFECTS IN CHILDREN}

$\lfloor$ Chen, C Anderson, E Rosko

Children's Hospital \& Regional Medical Center - Seattle, Seattle, Washington, USA

INTRODUCTION: Morphine and hydromorphone delivered via patient controlled analgesia is one of the primary means of treating acute pain in hospitalized patients. In the pediatric population, few studies have been performed reviewing the incidence of side effects caused by the two medications.

METHODS: We searched our in-house medical database for patients on the Acute Pain Service who used morphine or hydromorphone PCA's between December 11, 2003 and December 11, 2005; the resulting days of respective PCA use were totalled. The number of days any side effect was present was noted and tallied.

RESULTS: A total of 1834 days of morphine PCA use by patients was noted. 1185 days $(64.61 \%)$ had no listed side effect. Of the top three side effects, nausea and/or vomiting was noted on 471 days $(25.68 \%)$, pruritis on 310 days $(16.9 \%)$, and urinary retention on 15 days $(0.82 \%)$. A total of 660 days of hydrmorphone PCA use by patients was noted. 372 days $(56.36 \%)$ had no listed side effect. Nausea and/or vomiting was noted on 228 days (34.55\%), pruritis on 158 days (23.94\%), and myoclinic limb movements on 6 days $(0.91 \%)$. The totals do not add up as some patients had more than one side effect on each day.

DISCUSSION: The efficacy and safety of PCA use in the inpatient setting has been demonstrated in the pediatric population. However, no study has specifically compared the efficacy and side effects of morphine
PINC, DONOT COPY

and hydromorphone in the pediatric population. Anecdotal experience among our group of practitioners has given the impression that hydromorphone had a lesser incidence of side effects. Our preliminary data in this pilot study does not appear to support this perception. Of course many confounding factors exist, including the potential for erroneous charting from an older version of our current database, and the retrospective design of this study.

\section{REFERENCES}

1. Rodgers et al. J Pediatr Surg 1988;23:259-62.

2. Coda et al. Pain 1997;72:333-46.

3. Till et al. Paediatr Anaesth 1996;6:29-32.

\section{Diagnosis, Assessment}

\section{G1}

BACK PAIN IN CHILDREN NEEDS A CAREFUL EVALUATION E Ghayem Hasankhani, M Payvandi Mashad University of Medical Sciences, Mashad, Iran

BACKGROUND: Back pain in children has a high incidence of definable cause of symptoms, and back pain with unknown pathology is rare. The aim of this study is to determine the high incidence of definable pathology and necessity of careful evaluation of back pain in children.

MATERIALS \& METHODS: A retrospective study of 55 patients (22 female, 33 male) with back pain were seen between May 1999 and February 2004. the mean age was $9 / 5$ years ( $7-15$ years).

RESULTS: The pathology of back pain in these patients was as follows: vertebral tumours ( 3 patients), infection ( 3 patients), spondylolisthesis ( 5 patients), deformity of spine ( 8 patients), Scheuermann's disease (6 patients), disc hernia (4 patients), spinal dysraphism (3 patients).

Trauma (12 patients) and unknown pathology (nonorganic) (11patients), only 11 patients (20\%) had back pain with unknown pathology, contrary to adults with up to $70 \%$ unknown pathology back pain.

CONCLUSION: Back pain in children has high incidence of definable pathology $(80 \%)$ and radiographical and laboratory evaluation must be performed in children with back pain.

\section{G2}

\section{SCREENING INSTRUMENT FOR PEDIATRIC ADVANCED NEEDS ASSESSMENT (PANDA)}

I Cohen, M Silva, D LaFonde

Children's National Medical Center, Washington, District of

Columbia, USA

INTRODUCTION: Nurses and physicians caring for children with lifethreatening illnesses face many obstacles. The misunderstanding of the term palliative care and resistance to its practitioner's early involvement persists. The American Academy of Pediatrics states that, "Palliative treatments focus on the relief of symptoms (eg, pain, dyspnea) and conditions (eg, loneliness) that cause distress and detract from the child's enjoyment of life." (1) Early identification of children who would benefit from interdisciplinary approach to physical, emotional, and spiritual needs is warranted (2).

METHODS: The Palliative Care Committee at Children's National Medical Center in response to the needs of our high-risk population has formed a consultative service entitled the Pediatric Advanced NeeDs Assessment (PANDA) Care Team. To fulfill its stated purpose, the team researched and piloted a screening tool to identify patients in need of their services. Three practitioners tested the instrument (Table 1) for baseline validity and reliability. The interrater agreement among the three independent observers was determined using Cohen's Kappa statistic (3).

RESULTS: Twenty patients were interviewed and their charts were reviewed. All patients had been admitted to the Hematology/Oncology unit for sickle cell anemia vasoocclusive crises or cancer-related care. Content validity was assumed at this time because the instrument was adapted from well-established screening tools $(4,5)$. Kappa statistic for observer agreement was 0.74 .

DISCUSSION: Initial results demonstrate that the PANDA screening 
tool has potential for generalized use to determine if early intervention by reliability is needed but preliminary results are encouraging.

\section{REFERENCES}

1) American Academy of Pediatrics. Palliative Care for Children. Pediatrics 2000;106:351-7.

2) Center to Advance Palliative Care. Making the Case for HospitalBased Palliative Care. Accessed on December 12, 2005 at http://www.capc.org/building-a-hospital-based-palliative-careprogram/case/index html.

5) Cohen J. A coefficient of agreement for nominal scales. Educational and Psychological Measurement 1960;20:37-46.

4) Schag CC, Heinrich RL, Ganz PA. Karnofsky performance status revisited: Reliability, validity, and guidelines. J Clin Oncology 1984;2:187-93

5) Lansky SB, List MA, Lansky LL, Ritter-Sterr C, Miller DR. The measurement of performance in childhood cancer patients. Cancer 1987;60:1651-6.

\section{Table 1}

\section{PANDA screening tool}

\begin{tabular}{|c|c|c|}
\hline $\begin{array}{l}\text { CRITERIA } \\
\text { Disease process }\end{array}$ & Descriptors & $\begin{array}{l}\text { SCORE } \\
2 \text { points each }\end{array}$ \\
\hline Cancer & Metastatic or recurrent & \\
\hline AIDS/SCIDS & End stage & \\
\hline Cystic fibrosis & End stage & \\
\hline Cardiomyopathy & Moderate to severe & \\
\hline Renal failure & End stage & \\
\hline Hepatic failure & End stage & \\
\hline Neuromuscular diseases & End stage & \\
\hline Edocrine disorders & Unresponsive & \\
\hline Metabolic diseases & Unresponsive & \\
\hline Concurrent diseases & Descriptors & 1 Point Each \\
\hline See above & Mild to moderate & \\
\hline Functional status & Descriptors & Points \\
\hline Lansksy play scale & $<16$ years old & \\
\hline 100 & Fully active & zero \\
\hline 90 & Minor restrictions & zero \\
\hline 80 & Easily tires & zero \\
\hline 70 & Restricted & 1 \\
\hline 60 & Limited & 1 \\
\hline 50 & Assisted & 1 \\
\hline 40 & Quiet play only & 2 \\
\hline 30 & Assisted quiet play & 2 \\
\hline 20 & Passive activities & 2 \\
\hline 10 & No play & 3 \\
\hline Karnofsky scale & $>16$ years old & \\
\hline $100-90$ & Normal activities & zero \\
\hline $80-60$ & Requires assistance & 1 \\
\hline $50-40$ & Disabled & 2 \\
\hline $30-10$ & Moribund & 3 \\
\hline
\end{tabular}

\section{G3}

\section{THE EVALUATION OF FLACC (FACE, LEGS, ACTIVITY, CRY, CONSOLABILITY)}

\section{$\underline{\text { S Nilsson }}$}

Queen Silvia Children`s Hospital, Göteborg, Sweden, Göteborg, Sweden

BACKGROUND: Merkel et al (1997) evaluated, ages 0-7 years, the reliability and validity of the FLACC-scale which incorporates five categories of pain behaviours: facial expression; leg movement; activity; cry; and consolability. Each parameter is scored 0-2, for a total score of $0-10$. Willis et al (2003) found significant and positive correlation between the FLACC and self-report scores for the scores of children 5-7 years of age. In which age will a significant and positive correlation between the FLACC and self-report scores end?
AIM: The aim is to evaluate the reliability and validity of the FLACCscale for children, 5-18 years, undergoing procedural pain.

METHODS: Children were recruited from a pediatric oncology clinic and a surgery clinic at a Children's hospital.

Children (ages 5-18 years) who needed subcutaneous venous port device or a venous catheter were randomly assigned.

The child and the parents rated the affective dimension of pain using Facial Affective Scale (FAS) and the intensity of the pain using Coloured Analogue Scale (CAS) (McGrath et al, 1996).

The validity was evaluated with Kappa and the reliability was evaluated with Spearman correlation.

RESULTS: Preliminary results will be presented.

\section{REFERENCES}

McGrath PA, Seifert CE, Speechley KN, Booth JC, Stitt L, Gibson MC. A new analogue scale for assessing children's pain: An initial validation study. Pain 1996;64:435-43.

Merkel SI, Voepel-Lewis T, Shayevitz JR, Malviya S. The FLACC: A behavioral scale for scoring postoperative pain in young children. Pediatric Nursing 1997;23:293-7.

Willis MH, Merkel SI, Voepel-Lewis T, Malviya S. FLACC Behavioral Pain Assessment Scale: A comparison with the child's self-report. Pediatric Nursing 2003;29:195-8.

\section{G4}

\section{A SYSTEMATIC REVIEW OF MEASUREMENT INSTRUMENTS USED WITH PARENTS OF ADOLESCENTS EXPERIENCING CHRONIC PAIN}

A Jordan, C Eccleston

University of Bath \& RNHRD, Bath, United Kingdom

INTRODUCTION: Recent research has focused on the influence of pediatric chronic pain on the family, specifically focusing on the experience of parents as primary caregivers. Studies have indicated that caring for an adolescent with chronic pain negatively impacts on numerous areas of parental life and functioning. Measurement of this impact is a significant clinical and scientific starting point.

METHOD: We reviewed the use of instruments to assess the parental impact of caring for an adolescent with adolescent chronic pain. The review focused on the development of the measures, their psychometric properties and their use in studies of parents of adolescents with chronic pain.

RESULTS: Systematic literature searching recovered a total of 4225 articles, yielding a total of 74 articles which met eligibility criteria for inclusion in the review. Examination of these studies revealed the existence of 71 separate measurement instruments, all of which were subjected to detailed content analysis. The majority of instruments were found to be in the psychological $(n=33)$ or social domain $(n=25)$. 65 measures included a self-report format. The median number of items was 36. The median frequency of usage of instruments in the identified studies was 1 . Although 27 instruments had undergone psychometric evaluation in a sample of parents of adolescents with chronic pain, 20 of these evaluations solely involved assessment of internal consistency.

DISCUSSION: Clinically relevant psychometric data were missing for the majority of instruments, with a particular dearth of information regarding validity of the measures. The findings of this review demonstrated a huge diversity of instrumentation with very few pockets of depths of use. Results also identified poor or non-existent description of the demographic characteristics of parental samples in a number of studies. Findings support the need to further develop the knowledge base of measurement of the parental impact of chronic adolescent pain. 
G5

\section{THE DEVELOPMENT AND PSYCHOMETRIC EVALUATION \\ OF THE BATH ADOLESCENT PAIN PARENTAL IMPACT QUESTIONNAIRE (BAP-PIQ), AN INVENTORY TO MEASURE THE PARENTAL IMPACT OF CARING FOR AN ADOLESCENT WITH CHRONIC PAIN}

A Jordan, C Eccleston, L McCracken, H Connell, C Sourbut, J Clinch University of Bath \& RNHRD, Bath, United Kingdom

INTRODUCTION: Adolescent chronic pain has an extensive deleterious impact on adolescent and familial functioning. As primary caregivers, recent research has focused on examining the parental experience of caring for an adolescent with chronic pain. Initial findings demonstrate impaired levels of parental social and emotional functioning. Despite an increasing focus on parental experience, no psychometrically sound multidimensional assessment tools exist to assess the parental impact of adolescent chronic pain. This study reports on the development and evaluation of the Bath Adolescent Pain Parental Impact Questionnaire (BAP-PIQ), an inventory designed for use with parents of adolescents with chronic pain.

METHODS: A sample of 180 parents of adolescents (11-18 years) experiencing chronic pain completed the newly developed 94 item draft version of the BAP-PIQ. Participants were recruited from three tertiary centre clinic sites in the United Kingdom. In addition to the BAP-PIQ, participants also completed a number of additional questionnaires for validation purposes and basic demographic information..

RESULTS: Psychometric evaluation of the data was used to inform the elimination of redundant items from the BAP-PIQ. Internal consistency of all 8 subscales was established using Cronbach's alpha. The convergent validity of BAP-PIQ subscales was evaluated by means of correlation with existing standardised measures (BDI, PIP, PSI-SF, IBES, SF36v2, DAS). Temporal reliability of all 8 BAP-PIQ subscales was established over a two week period using a subsample of 50 participants.

CONCLUSIONS: Analysis of the data demonstrated the BAP-PIQ to be an appropriate, reliable and valid tool for assessing the parental impact of caring for an adolescent with chronic pain. The BAP-PIQ is a promising development for future research investigating the parental impact of adolescent chronic pain.

This research is funded by a grant provided by The BUPA Foundation.

\section{G6}

COGNITIVE AND ACADEMIC ACHIEVEMENT PROFILES OF CHILDREN AND ADOLESCENTS WITH CHRONIC PAIN $\underline{\mathrm{G} \mathrm{Ho}}^{1}$, S Bennett ${ }^{2,3}$, D Cox ${ }^{1}, \mathrm{G} \mathrm{Poole}^{4}$

1Department of Psychology, Simon Fraser University, Burnaby; ${ }^{2}$ Integrated Pain Service, BC Children's Hospital; ${ }^{3}$ Department of Psychology; ${ }^{4}$ Department of Health Care and Epidemiology, The University of British Columbia, Vancouver, British Columbia In adults, chronic pain and associated disability are known to have staggering personal and economic costs. Less is known about the impact of chronic pain in childhood. For children and adolescents, school functioning is a key aspect of pain-associated disability. The costs of missed school can alter the life of a child. Thus, evaluation of factors affecting learning and school adjustment is an essential component of a comprehensive assessment of children and adolescents presenting with chronic pain. To date, systematic empirical data on the cognitive and achievement profiles of pediatric chronic pain patients are lacking. This retrospective chart review study documented the cognitive functioning and academic achievement of a clinical sample of 57 children and adolescents with chronic pain, using standardized test batteries including the Wechsler scales. Participants were 8 to 18 years old (mean age $=14.64 ; 81 \%$ females) and were seen in a tertiary care interdisciplinary pain program between 1998 and 2004. Results indicated that the group means on measures of general cognitive abilities and visual-motor skills fell within the Average range. Group means for general intelligence, verbal ability, nonverbal reasoning ability and processing speed scores were significantly higher than those of the general population. Among those with significant intraindividual variability within their cognitive profile, most had stronger verbal ability.
Most frequently occurring relative weaknesses were in working memory or processing speed. Mean academic achievement was in the average range on all of the scholastic domains covered, with a subset of children and adolescents scoring significantly higher in word reading, reading comprehension and written expression, but worse in arithmetic computation, than the general population. Future work is needed to examine the relationship between pain, psychoeducational testing results, school attendance and other indices of adjustment.

\section{G7}

\section{PAIN AND SYMPTOM EXPERIENCES IN HOSPITALIZED CHILDREN WITH CANCER}

$\underline{\text { E Jacob }}^{1}$, J Hesselgrave ${ }^{2}$, G Sambucco ${ }^{3}$, M Hockenberry $^{4}$

${ }^{1}$ Baylor College of Medicine; ${ }^{2}$ Texas Children's Cancer Center;

${ }^{3}$ Texas Children's Hospital; ${ }^{4}$ Texas Children's Hospital Center for Clinical Research and Evidence Based Practice, Houston, Texas, USA PURPOSE: Even though pain and its effects in children with cancer are now better understood, it is often still not managed optimally. Children with cancer may also experience symptoms other than pain that may affect treatment outcomes. We examined the pain and symptoms experienced by 38 children with cancer, who provided data during a combined total of 132 days.

METHODS: Children ( 8 to 17 years), diagnosed with cancer, and hospitalized in the hematology/oncology unit at Texas Children's Hospital were asked to complete the Adolescent Pediatric Pain Tool, the Memorial Symptoms Assessment Scale, the Pediatric Quality of Life instrument. Children completed the instruments within 24 hours of admission up to 5 days during hospitalization.

RESULTS: Pain was experienced during $56.1 \%$ of the days ( 74 days). The mean pain intensity score on a 0 to $10 \mathrm{~cm}$ Word Graphing Rating Scale was $4.2, \mathrm{SD} 3.0$. The mean total number of areas marked on the body outline diagram was 3.5, SD 3.0. The most frequent locations marked were the abdomen ( 23 days) and lower back (15 days). The mean total number of words chosen for each day of the episode was 10.0, SD 10.0. The most frequently used words to describe the quality of their pain were uncomfortable (42 days), annoying (33 days), sore (22 days), awful (21 days), comes and goes (20 days), and stiff (19 days).

Children not only reported pain, but they also reported multiple symptoms during hospitalization. The most frequently reported symptoms were lack of energy (41 days), nausea (36 days), lack of appetite or not wanting to eat (35 days), cough (27 days), feeling of being drowsy ( 27 days), feeling of sadness ( 23 days), worrying ( 23 days), itching ( 23 days), difficulty sleeping (21 days), and change in the way food tastes ( 25 days).

CONCLUSION: Results of this study increased our understanding of the frequency, severity, trajectory, and impact of symptoms in children with cancer. This information may be used to initiate interventions that target both individual symptoms and concurrent symptoms. Treatment outcomes in relation to pain and symptoms are extremely important, but as yet relatively unexplored in children with cancer. Many cancer patients experience several symptoms concurrently and may affect multiple patient outcomes, including morbidity, functional status, and quality of life. Although underlying mechanisms of concurrent symptoms are not yet understood, a framework may be developed for clinicians to use in considering selection of interventions, and in weighing the costs and benefits of those interventions.

\section{G8}

FOLLOW-UP WITH THE DENTAL DISCOMFORT QUESTIONNAIRE FOR YOUNG CHILDREN BEFORE AND AFTER DENTAL TREATMENT

IVersloot, J Veerkamp, J Hoogstraten

Academic Centre for Dentistry Amsterdam (ACTA), Amsterdam, The Netherlands

Dental caries in young children (3-5 y) can possibly lead to toothache and as a consequence can be associated with reduced quality of life. Young children do not necessarily complain verbally on dental pain or toothache which makes its recognition difficult at that age. Therefore the dental discomfort questionnaire (DDQ) was developed. 
The aim of the present study is twofold: first to assess the test-retest reliability of the DDQ and second to perform a follow-up study to assess the effect of dental treatment on pain related behaviours. The DDQ is a 9-question instrument (eg, does your child have problems chewing?) to assess dental pain related behaviours in young children (age 22-59 months). For the test-retest analysis, the questionnaire was filled out twice before treatment by 44 parents on behalf of their child. For the follow-up study the questionnaire was filled out by 71 parents before and after all dental disease was treated under general anesthesia.

The test-retest reliability proved very satisfactory over a two month period. When scores on the 9 behaviour items are compared before and after treatment it appeared that after treatment all but one behaviour (ie, bites with molar instead of teeth) were displayed less often. Overall, after treatment there was a significant lower mean DDQ score, thus the mean number of pain-related behaviours displayed decreased after treatment.

Dental treatment of children leads to a reduction of toothache related behaviours and probably to a better quality of life. The DDQ is a sensitive instrument to measure dental discomfort before and after restorative treatment underlining the importance of a behavioural approach in young children. Finally, the DDQ can possibly support healthcare providers, pediatricians and parents in their assessment of toothache in young children.

\section{G9}

\section{DEVELOPMENT OF THE COPING WITH NEEDLES QUESTIONNAIRE FOR CHILDREN: EXAMINING COPING DIMENSIONS USING CONFIRMATORY FACTOR ANALYSIS}

\section{Spagrud, L Besenski, C Hammond, C von Baeyer} University of Saskatchewan, Saskatoon, Saskatchewan

The investigation of children's reactions to painful medical procedures has broadened from a focus on inducing compliance with medical procedures to an expanded focus on studying how children cope. This trend reflects increased recognition of the distress associated with both traumatic and more routine medical procedures, and the risks associated with forced compliance (eg, avoidance of future medical care). The goal of the present investigation is to use the transactional model of coping to develop a questionnaire to assess coping behaviour in children undergoing immunization. Use of this definition necessitates an understanding that individuals may possess a whole range of coping strategies; however, their presence does not necessarily indicate how or when they will make use of them. Therefore, measurement of coping strategies should be situation- or stressor-specific, rather than a general assessment of coping style. Although many measures of both general coping style and coping with other identified stressors (eg, chronic pain) already exist, there is a need for the development of a measure that specifically assesses children's coping with needles. Approximately 300 children (age 11-12) receiving hepatitis B immunizations will be asked to complete the Coping with Needles Questionnaire (CNQ). The CNQ is a self-report scale that asks children to rate how much ( $1=$ very unlike me to $5=$ very like me) each of 42 statements reflects how they coped with a needle. Data will be analysed using confirmatory factor analysis to examine whether or not the items of the CNQ adhere to the dimensions of coping discussed in the literature (ie, approach versus avoidant coping strategies). The resulting CNQ will be used in a future research project investigating the relationship between children's self-reported coping behaviour and repeated immunization pain.

\section{G10}

BEHAVIOURAL AND PHYSIOLOGICAL CORRELATES OF CHILDREN'S SELF-REPORTED PAIN: A PILOT STUDY

R Foster $^{1,2}$, J Zuk ${ }^{1,2}$, D Polaner ${ }^{1,2}$, J Carollo ${ }^{2}$, L Stallwood ${ }^{1,3}$, K Montgomery ${ }^{2}$

${ }^{1}$ University of Colorado at Denver; ${ }^{2}$ The Children's Hospital;

${ }^{3}$ Health Sciences Center, Denver, Colorado, USA

AIMS: To examine correlations among linked self-report, behavioural, and physiological data to guide hypothesis development.

BACKGROUND: Effective pain management depends on the clinician's ability to identify the presence of pain and assess its intensity. Assessment is
STM

enhanced when children can verbally describe their pain or use a pain scale. But what about children who cannot or who choose not to communicate about pain? Can behavioural and/or physiological cues be used reliably in the absence of self-report? Can clinicians confidently prescribe or administer potent analgesics on the basis of these assessments? The purpose of this study is to pilot methods to collect specific behavioural and physiological data that can be examined for correspondence with children's self-report.

METHODS: The study will use a one-group, repeated measures design. Key variables comprise of physiological data (computer-downloaded physiological output for heart rate variability, respiratory rate, $\mathrm{SpO} 2$, and blood pressure), self-report (Bieri Faces Scale-Revised and the Affective Facial Scale), and behavioural cues (digital video of the child's face and upper body coded for pain cues using the Child Facial Coding System, and the FLACC behavioural pain scale). Up to 30 children will be recruited to achieve complete data for 20 children. Inclusion criteria specify children must have acute, established pain that varies in intensity. Data collection will occur every 15 minutes over a 3 -hour period or until 8 measurements have been completed for physiological, self-report, and behavioural variables. Descriptive data will include pain medications, personal characteristics, and environmental influences (including decibel readings for environmental noise).

RESULTS: Data collection is in process. Descriptive and correlational data will be presented. The research will generate hypotheses for a larger study.

Funding: This research was supported by grant number 5 M01 RR00069, General Clinical Research Centers Program, National Center for Research Resources, NIH and The Children's Hospital Research Institute, G06869.

\section{G11}

MEASURING PAIN IN YOUTH WITH DISABILITIES: NUMERICAL RATING SCALE

\section{R Ballard, L Engel, D Amtmann}

University of Washington, Seattle, Washington, USA

Self-reports are the "gold standard" in pain assessment. This study examined the use of a numerical rating scale (NRS) to self-report pain intensity by youths with disabilities. The advantages of NRS include its ease in administration and clinical utility. Currently the NRS is not commonly used with children in published research. In this study we investigated whether youth with disabilities could successfully use the NRS and we examined the relationships among different types of pain intensity ratings. 112 participants, aged $8-20$ years $($ mean $=14.2, \mathrm{SD}=1.24)$, were asked to use an 11-point NRS to rate their current pain, least, worst, and average pain over the past week.

There were no significant differences by sex or disability in any of the four pain intensity ratings. 13 participants reported zero pain for each rating. 14 participants gave illogical answers, eg, least, worst, and current pain ratings were 0 while the average pain rating was 5 . Worst pain and pain variability were statistically significant predictors of average pain, $\mathrm{F}(1,108)=135.2, \mathrm{P}<0.01$ and $\mathrm{F}(1,108)=46.03, \mathrm{P}<0.01$, respectively. Worst pain accounted for about half of variance of average pain $\left(\mathrm{r}^{2}=0.56\right)$ and pain variability explained about one third of the variance of average pain $\left(r^{2}=0.30\right)$. The combination of either worst pain with least pain or variable pain with least pain accounted for the same amount of variance in average pain, approximately $60 \%\left(r^{2}=0.59\right)$.

Study findings support the use of the NRS with youths with disabilities. The worst pain appeared to be heavily considered when youths reported average pain and some youths had difficulties with ratings of average pain. Consistent and valid data can be collected by asking about current, least, and worst pain.

\section{G12}

THE IMPACT OF SCALE FORMAT ON CHILDREN'S AND PARENTS' PAIN RATINGS

$\underline{L}^{\text {Uman }}{ }^{1,2}$, C Chambers ${ }^{1,2}, \mathrm{C}$ von Baeyer ${ }^{3}$

${ }^{1}$ Dalhousie University; ${ }^{2}$ IWK Health Centre, Halifax, Nova Scotia;

${ }^{3}$ University of Saskatchewan, Saskatoon, Saskatchewan

Many self-report scales for assessment of children's pain have been published, 
including numerical rating scales and faces scales. The format of faces scales influences pain intensity ratings: scales anchored by a smiling face to represent no pain produce higher pain ratings than scales beginning with a neutral face. The clinical significance of these differences has been demonstrated with nurses but has not yet been tested for parents or children. The purpose of the present study was to examine the impact of scale format on children's and parents' pain intensity scores using faces and numerical scales. Participants included 48 children ( $8-12$ years) and one of their parents who independently rated pain in three vignettes depicting either injury, disease-related, or postoperative pain. They rated the amount of pain they (or their child) would have to be experiencing before asking for help (an indicator of clinically significant pain), using a series of three scales which were presented in a counterbalanced order: (1) printed Numerical Rating Scale (NRS, 0 to 10, with 11 possible responses); (2) Wong-Baker FACES scale (WB, smiling zero-pain face, with 6 possible responses); (3) Faces Pain Scale - Revised (FPS-R, neutral zero-pain face, with 6 possible responses). As hypothesized, parents and children gave higher ratings on the WB compared to the FPS-R and NRS for all 3 vignettes. Repeated measures ANOVAs for parents and children, collapsing across vignettes, showed that all 4 predicted comparisons were statistically significant $(\mathrm{p}<.03)$. These results are consistent with past research showing that scales beginning with a smiling face can provide overestimates of pain, and suggest that self-report scale format can affect how parents and children rate clinically significant pain.

\section{G13}

DECODING CUES: HOW PARENTS KNOW WHEN THEIR CHILD IS IN PAIN

$\underline{\text { R Zisk }}^{1,2,}$ M Grey ${ }^{1}$, Z Kain ${ }^{3}$

${ }^{1}$ Yale University School of Nursing; ${ }^{2}$ Department of Anesthesia; ${ }^{3}$ Departments of Anesthesiology, Pediatrics, and Child Psychiatry, Yale University School of Medicine, New Haven, Connecticut, USA PURPOSE: Pain is a multidimensional phenomenon that can be expressed in many ways. Although self-report is considered as the gold standard when assessing pain, children do not always tell their parents when they are in pain. Thus, parents have to base management decisions on both verbal and behavioural cues. The purpose of this study was to examine parental perceptions regarding their children's pain expressions.

METHODS: This cross-sectional study examined 110 parents of healthy children ages 3-14 undergoing elective surgery. Nine statements, rated on a 7-point Likert scale, were developed using existing data, and were examined by a panel of experts to establish content validity. Data analysis included descriptive statistics, factor analysis and Cronbach's Alpha.

RESULTS: Over $80 \%$ of the parents thought that children in pain have trouble sleeping, $44 \%$ agreed that children always express pain by crying or whining, $43 \%$ agreed that children exaggerate pain, 38\% thought that children always tell their parents when in pain, and $31 \%$ thought that children complain about pain to get attention. A factor analysis with Varimax rotation suggested a 3 -factor solution, explaining $69 \%$ of the variance. The factors were active, loud behaviours, attention seeking and quiet, withdrawn behaviours. The Cronbach's Alpha of 0.79 suggested internal consistency between the items.

CONCLUSIONS: Children express pain in a variety of ways. Parents were not aware of all possible pain expressions children may exhibit. Further validation of the instrument is needed, as well as studies to examine the effect of parental perception of children's pain expression on actual parental pain management practices at home.

\section{G14}

PERCEPTION OF HEALTH CARE PROFESSIONALS ABOUT THE SICKLE CELL PAIN CRISIS

E Record $^{1}$, B Carroll ${ }^{2}$, R Pitts ${ }^{2}$

${ }^{1}$ Children's Healthcare of Atlanta, Marietta; ${ }^{2}$ Children's Healthcare of Atlanta, Atlanta, Georgia, USA

BACKGROUND: The hallmark of Sickle Cell Disease (SCD) is the vasoocclusive crisis (VOC), or pain crisis. This is the number one cause of hospital admissions for patients with SCD and is also the most feared manifestation for many SCD patients.

OBJECTIVE: To assess the current perceptions of health care professionals regarding sickle cell pain.

METHODS: This study was conducted at Children's Healthcare of Atlanta. Surveys were given prior to an inservice on SCD to 100 health care personnel.

RESULTS: 90 (90\%) of those responding believed that MD's and RN's underestimate the degree of sickle cell pain. In addition, $38(38 \%)$ of respondents believe that the incidence of addiction to narcotics in sickle cell patients ranges from $10->50 \%$. The incidence of addiction in SCD is only between $0.2 \%$ and $2 \%$. Another question asked respondents to evaluate their frustration/stress when providing care to four different types of patients. $73(73 \%)$ identified the sickle cell patient with pain as the most stressful/frustrating. Finally, 19 (19\%) of respondents do not believe that sickle cell patients have chronic pain.

DISCUSSION: Prompt, adequate and consistent treatment of the VOC in SCD is the responsibility of the health care professional. However, if health care professionals are encumbered with inaccurate beliefs, the incidence of addiction or are frustrated /stressed when providing care to sickle cell patients or if health care professionals frequently underestimate the degree of sickle cell pain, the treatment of the VOC will fail. It is incumbent upon those most knowledgeable about SCD to continuously strive to educate health care professionals to dispel the misconceptions or myths about the pain of SCD and the effects of such pain.

\section{G15}

\section{INSOMNIA SYMPTOMS IN ADOLESCENTS WITH CHRONIC PAIN: AN ACTIGRAPHIC STUDY}

I Palermo, I Fonareva, J Koh

Oregon Health and Science University, Portland, Oregon, USA

INTRODUCTION: Many adolescents with chronic pain report sleep difficulties. There has been limited description, however, of the specific nature of these sleep difficulties. The purpose of this study was to characterize sleep in adolescents with chronic pain using subjective and objective sleep measures and to specifically examine the prevalence of insomnia symptoms.

METHODS: Seventeen adolescents (mean age $=14.4,65 \%$ female) with chronic headaches, myofascial pain, or limb pain for at least 3 months $(\mathrm{M}=1.8$ years $)$ participated in the study. Teens completed measures of pain severity, sleep quality, presleep arousal, and depressive symptoms. Actigraphy was completed over 7 days to detect sleep-wake patterns. Actigraphic sleep measures were aggregated for analyses. We defined insomnia as sleep onset latency of at least 30 minutes, and poor sleep as an average of at least 3 nightly awakenings lasting 5 minutes and/or sleep per cent below $90 \%$.

RESULTS: Overall, participants' total sleep times were short (mean $=6 \mathrm{hr} 56 \mathrm{~min}$ ). Participants had low sleep efficiency (mean $=74.1 \%)$, long sleep onset $($ mean $=35.4 \mathrm{~min})$, and frequent night wakings (mean $=4.4)$. Sixteen $(94.1 \%)$ adolescents met criteria for poor sleep and 7 (41.2\%) displayed insomnia symptoms. Higher number of presleep unpleasant somatic sensations (eg, heart pounding) was associated with more night awakenings $(r=0.39)$. Depressive symptoms were significantly associated with unpleasant pre-sleep somatic $(r=0.65)$ and cognitive $(r=0.69)$ sensations. Pain symptoms were not related to actigraphic sleep measures.

CONCLUSIONS: Poor sleep and insomnia are common in adolescents with chronic pain. Pain severity was not independently associated with sleep, suggesting that transient sleep problems may become chronic, independent of pain severity, due to increased presleep physical sensations/cognitions incompatible with sleep. Future plans are to enroll a comparison group of healthy adolescents to explore differences in both the prevalence and presentation of insomnia symptoms. 
G16

\section{PAIN AND QUALITY OF LIFE IN CHILDREN WITH SICKLE CELL DISEASE}

\section{T Murphy}

Department of Psychologial Medicine, London, United Kingdom

Pain is a common symptom in Sickle Cell Disease (SCD). In the past literature on SCD has investigated pain as a single variable. More recent studies have looked at pain in relation to other variables in the lives of children with SCD. Quality of life is a comprehensive measure of functioning and one which is growing within the pediatric psychology. Fiftyone children with SCD took part in the current study, 27 girls and 24 boys. The mean age was 11.31 years (range $7.8-15$ years, sd 2.29). Forty-four children had Sickle Cell Anemia (HbSS), nine children had Sickle Cell Disease (HbSC) and one child had Sickle Cell Thalassemia ( $\mathrm{Hb}$ beta thal).Participants in the SCD group were attendees at Sickle Cell Clinics in two South London hospitals. Measures of pain (diary-recorded and estimated pain) indicated that the frequency of pain events varied widely within the SCD group. Estimated pain was found to be lower than diary reported pain during a two week period. Estimates of pain significantly predicted functioning, satisfaction and upset in QoL. Diary-recorded pain was not found to be associated with QoL variables. Descriptives of pain were reported. Diary pain measures provide useful information on frequency, severity and location of pain. Estimated pain measures provide insight into the children's perception of their pain and perceptions of QoL. The two pain measures provide independent and perspectives information on pain use different processes to understand a child's experience of pain.

\section{G17}

\section{FACTORS AFFECTING TIME TO FIRST SICKLE CELL PAIN EPISODE IN YOUNG CHILDREN WITH SICKLE CELL DISEASE}

B Ely $^{1,2}$, C Dampier $^{1,2}$, D Brodecki ${ }^{2}, \mathrm{C} \mathrm{Coleman}^{2}, \mathrm{P} \mathrm{O}^{\prime} \mathrm{Neal}^{2}$ ${ }^{1}$ Drexel University College of Medicine; ${ }^{2}$ St Christopher's Hospital for Children, Philadelphia, Pennsylvania, USA

Using a longitudinal design, parents reported pain occurrence, location, associated symptoms and treatment provided during home-based pain episodes in young children with sickle cell disease (SCD). Pain-related data were collected using either daily paper diaries, daily calendars, or most recently, with an electronic daily pager system. Serial hematological values were also obtained at 3 month intervals, at least 2 weeks from any clinical event. Data reported here are from 55 children (24 SS, 45\%; 21 SC, 38\%; $8 \mathrm{~S} \beta+$ Thal; $14.5 \% ; 2 \mathrm{~S} \beta 0 \mathrm{Thal} ; 3.6 \%)$ less than 16 months of age at enrollment $(\mathrm{M}=0.52 \pm 0.27$ months) who had had no previous sicklerelated pain symptoms. After a median observation period of 15 months, the parents of 32 children reported a first sickle cell-related painful episode. Average age at first reported pain episode for these 32 children was $1.8 \mathrm{yrs}(\mathrm{SD}=1.46 \mathrm{yrs})$ and $17(53.1 \%)$ of the 32 children were homozygous SS. Kaplan-Meier analysis estimated a median time to first painful episode of any type of 929 days (95\%CI, 612-1819 days), and 957 days (95\% CI, 706-1826 days) for dactylitis-like episodes. Children with the SS genotype had a significantly shorter median time to first dactylitis episode than those with SC genotype (706 vs 1425 days, $\mathrm{P}=0.02$ ). First sickle pain episodes ranged in length from 12 hours to 8 days $(\mathrm{M}=2.77$ days, $\mathrm{Median}=2$ days $)$. The serial hematological data were analyzed as time dependent variables in a Cox-proportional hazard model of time to first painful event for children with SS or SC genotype. F-cell percentage, WBC count, and hemoglobin level were not significant predictors, but increased reticulocyte counts increased the hazard for a dactylitis episode $(\mathrm{P}=0.0006)$, irrespective of hemoglobinopathy diagnosis. These studies provide new insights into factors that predict risk of clinical pain events in infants and young children with SCD.
G18

\section{THE CHILD HEARING VOICE: A MISDIAGNOSED FREQUENT AUDITORY MIGRAINE AURA}

D Annequin, B Tourniaire, G Edith, T Anne, D Anne Claire,

G Mazaltarine, P Lacoste Munoz

Centre de la migraine de l'enfant, Paris, France

BACKGROUND: Occasional few cases of migraine aura as auditory hallucinations have been yet published (Rubin D, Headache 2002 Schreier HA. J Child Neurol 1998)

METHODS: During each initial consultation description of attacks, types of aura (visual, sensitive, auditory) have been systematically searched.

RESULTS: 970 consecutive children have been assessed over 17 months period. Mean age: $10.5 \pm 3.5$ years; $6.5 \%$ are less than 6 years; $29 \%$ are over 12 years. The diagnosis of migraine was carried to $95 \%$ of the children according to IHS 2004 criteria. Auditory aura was found for $27 \%$ of the children. 81 of these children described auditory hallucination as a "voice calling them": for $60 \%$ of children, it was the mother or a familiar voice. $42 \%$ of children declared that the voice frequency occurred "always" or "very often" with each migraine attack. This specific aura occurs during the headache in $50 \%$ of the cases and before the headache in $28 \%$ of the cases. Among the 81 children hearing voices, $69 \%$ visual aura and $50 \%$ sensitive aura features were also noticed. In $18 \%$ of the cases, the voices were associated other auditory perceptions (whistle, buzz...). In most of the cases this specific aura was discovered during the consultation. A child had been previously referred to psychiatric advice for psychosis suspicion.

CONCLUSION: Voice as auditory hallucination must be searched systematically in any child with migraine. This unexceptional specific sign could mislead to erroneous diagnostic as epilepsy or psychosis.

\section{G19}

\section{THE ADULTS' RESPONSES TO CHILDREN PAIN INVENTORY: DEVELOPMENT AND PRELIMINARY VALIDATION}

A Huguet, J Miró, R Nieto, J Baos, R Olivé, S Paredes Department of Psychology, Rovira i Virgili University, Tarragona, Spain

Forces in the environment are known to influence children's pain experience. Thus, reliable and valid instruments which let pain clinicians and researchers to assess the impact of those forces are needed.

The objective of our work is to present the development and preliminary validation of a questionnaire designed to assess the responses that adults give to children's pain.

Two of the authors (AH, JM) developed a comprehensive list of responses adults may enact in relation to children's pain experience, responses fall in three different categories (punishment of pain behaviour, reinforcement of pain behaviour, and prompting/reinforcing adaptative behaviours).

This version of the instrument was self-administered to parents of 250 children aged between 7 and 16 years.

Exploratory and confirmatory factor analyses show a robust structure of the inventory, based on the three factors previously mentioned. The internal consistency coefficients indicated that the three scales have good reliability, and the test-restest stability is adequately high (all greater than 0.70 ).

The results support the reliability of the inventory scales, and the validity of the instrument. However, further studies with different population are warranted to replicate the factor structure of the questionnaire, and to examine its predictive value.

This research was supported in part by grant FISO2/1353 from the Fondo de Investigaciones Sanitarias, Instituto de Salud Carlos III, Spanish Ministry of Health, and by the Agència de Gestió d'Ajuts a la Recerca, Department of Universities and Research, Generalitat de Catalunya. 
G20

\section{PROGRESSION OF PAIN INTENSITY, LOCATION AND QUALITY FOLLOWING SPINAL FUSION FOR IDIOPATHIC SCOLIOSIS}

\section{Kleiber ${ }^{1}$, L Dolan ${ }^{2}$, M Berg ${ }^{2}$, A Kleese ${ }^{2}$, M Suwanraj ${ }^{2}$}

${ }^{1}$ University of lowa College of Nursing; ${ }^{2}$ University of lowa, lowa City, lowa, USA

PROBLEM: Spinal fusion for correction of idiopathic scoliosis is one of the most painful surgeries performed. Physical therapy to strengthen and stretch muscle tissue begins just a few days after the surgery and this therapy continues for several months. The literature clearly describes the intensity of pain experienced after spinal surgery, but there are no descriptions of the quality and location of the pain. Also, little is known about how pain changes after the immediate postoperative period. This information is needed to develop the most appropriate pain prevention and treatment interventions for this group of children. The aim of this study is to describe the intensity, quality and location of pain experienced by adolescents over the first month after spinal fusion for idiopathic scoliosis.

METHODS: A prospective repeated measure descriptive design was used to describe children's pain intensity, location and quality over time, using the Adolescent Pediatric Pain Tool (APPT). This tool is a multidimensional instrument that consists of a body outline divided into 43 segments, a $100 \mathrm{~mm}$ word graphic scale with five equidistant pain intensity descriptors, and a pain quality descriptor list. Construct validity and reliability of this instrument has been established.

SAMPLE: Twenty-three English speaking children between the ages of 10 and 18, who were scheduled for repair of idiopathic scoliosis at a large Midwestern teaching hospital, were enrolled in this study. Children completed the APPT 5 times: once preoperatively to get a baseline measure, and again at 48 hours, 72 hours, 2 weeks and 4 weeks postoperatively. Data were also collected on the type and amount of pain medication taken.

DATA ANALYSIS: Results will be presented graphically to display change in intensity over time. Frequency of pain location and quality descriptors, and how these change over time, will be reported.

\section{G21}

\section{PAIN INCIDENCE IN 60 CHILDREN WITH LYSOSOMAL DISEASES}

F Reiter $^{1}$, B Tourniaire ${ }^{1}$, B Heron ${ }^{2}$, N Guffon ${ }^{3}$, D Annequin ${ }^{4}$ ${ }^{1}$ Unité Douleur Hopital Trousseau; ${ }^{2}$ Neuro Pediatry Hopital Trousseau, Paris; ${ }^{3}$ Metabolic Diseases, Edouard-Herriot Hospital, Lyon; ${ }^{4}$ Unite Douleur. Centre de la migraine de l'enfant Hopital Trousseau, Paris, France

INTRODUCTION: Lysosomal diseases are genetic evolutive disorders with a progressive motor and/or cognitive impairment and organic dysfunctions (heart, kidneys, gut, bone and joints... can be involved). Despite multiple types of pain in this population, literature is sparse.

PATIENTS AND METHODS: This prospective study was conducted in two French reference centres for lysosomal diseases. Interviews were conducted by an independent observer with an assessment of the different pain sites (joint, bone and muscle, abdomen, head, tooth...), characteristic features (neuropathic, nociceptive...), frequency, and treatment. A special interest was focused on pain related to physiotherapy, mobilization or wash. Pain was self assessed (VAS, faces scale, and body scheme) according to the child's cognitive ability; otherwise parents did the assessment with a VAS, and a specific French validated scale for cognitively impaired children, San Salvadour scale (DESS) Collignon (Eur J Pain 2001).

RESULTS: Sixty children were evaluated (medium age: 9.4 years). Self-assessment was possible for 21, and there was a high correlation with parents evaluation. Six out of 45 children who had an observational evaluation with DESS had high levels of pain $73,3 \%$ of children had pain in the previous weeks: limbs (86\%) and back (50\%) with a medium VAS at 5.5/10, abdomen (25\%), ENT (33\%), headache (27\%). Neuropathic pain was obvious in $18 \%$ and likely in $10 \%$ more. $70 \%$ had pain more than once a week or every day. Physiotherapy was frequent ( 3 times a week) and painful for $65 \%$ of these children: medium VAS 6.5/10. 60\% received a treatment for pain; this was prophylactically administered in only a quarter of them. Only six (10\%) had an opioid treatment and $10(16 \%)$ a treatment for neuropathic pain.

CONCLUSION: In this population, pain is frequent, underdiagnosed, and undertreated. The second step of this study will be to customize the best treatment for each child..

\section{G22}

RELATIONSHIP BETWEEN PATIENT SELF-REPORT AND DIAGNOSIS IN CHILDREN AND ADOLESCENTS WITH CHRONIC OR RECURRENT PAIN SYNDROMES

\section{A Feinstein ${ }^{1}$, G Walco ${ }^{1,2}$}

${ }^{1}$ Hackensack University Medical Center; ${ }^{2}$ University of Medicine and Dentistry of New Jersey, New Jersey Medical School,

Hackensack, New Jersey, USA

The purpose of this study was to ascertain if there is a relationship among divergent self-reported characteristics of chronic or recurrent pain and if there are differences in self-report based on the general type of pain complaint. Medical records of 188 children and adolescents between the ages of 5.6 and 20.6 years who were evaluated at an outpatient pediatric chronic pain program were reviewed retrospectively. The Pediatric Pain Questionnaire was utilized during the initial evaluation to assess various elements of the pain experience including: pain location based on a body diagram, pain quality derived from a list of sensory, affective and evaluative pain descriptors, and pain intensity from visual analogue scale ratings of present, average and worst pain in the past week. Results indicated that patients who reported a wide distribution of pain sites endorsed significantly higher numbers of sensory pain descriptors and that endorsement of sensory descriptors was correlated with endorsement of affective and evaluative pain descriptors. When the sample was divided into subgroups according to primary types of pain (musculoskeletal, abdominal, headache, and other) significant group differences for sensory descriptors and ratings of present and average pain intensity were found. Further analyses of the musculoskeletal pain group, which included fibromyalgia, rheumatological conditions, complex regional pain syndrome-type 1 (CRPS-1), and orthopedic conditions, showed that while as expected, patients with fibromyalgia had more global distribution of pain sites than patients with CRPS- 1 or orthopedic conditions, no significant differences were found in pain intensity ratings or the pain descriptors endorsed. The data suggest that children and adolescents with chronic pain who report a wide distribution of pain sites have a poor ability to localize their pain and their discriminative capacities may also be compromised. These findings have implications for assessment, differential diagnosis, and possible treatment strategies.

\section{G23}

\section{BACK PAIN FOLLOWING EPIDURAL ANALGESIA IN CHILDREN}

S Raghavendran ${ }^{1}$, H Bagry ${ }^{2}$, T Valois ${ }^{1}$, J Muir ${ }^{3}$, M Ranger ${ }^{1}$, J Brouillard ${ }^{1}, K$ Brown $^{1}$

${ }^{1}$ Montreal Children's Hospital; ${ }^{2}$ Montreal General Hospital, Montréal, Québec; ${ }^{3}$ Halifax Children's Hospital, Halifax, Nova Scotia BACKGROUND: Back pain following epidural analgesia has been extensively studied in the obstetric population with a reported incidence of $17.8-30 \%(1,2,3)$. The relationship between the two is complex and highly debated. Studies in the nonobstetric adult population have again yielded inconclusive results $(4,5)$. It may on the one hand be trivial but on the other hand it may be a manifestation of a serious underlying cause such as osteomyelitis, epidural abscess or a hematoma $(6,7)$. However, these risks are not clearly addressed in the pediatric population. We propose to address the issue of backache and its risks if any from neuraxial blocks administered to these patients.

OBJECTIVE: We aim to assess the incidence of early and late back pain in children following epidural analgesia and its relationship with age, gender, type of epidural technique and the level of insertion, gauge of needle used, number of attempts, position during surgery and operator factors.

METHODS: We enrol children who have received epidural analgesia for a surgical procedure. Consent is obtained from the parents prior to 
discharge from the recovery room. The parents are given a questionnaire and a telephonic inquiry is done on day 2 and day 15 postsurgery. Information obtained on day 2 assesses early back pain of possible inflammatory origin while on day 15 , the persistence of back pain will be considered delayed onset back pain of other possible origin. Patients with persistent back pain on day 15 are offered an appointment for further follow-up. A combination of parental report and self-report is used to record pain.

RESULTS AND DISCUSSION: All analyses will be done using SPSS and a $\mathrm{P}$ value $<0.05$ for significance. This study is in progress and results will be available by June 2006 with an interim report by April 2006.

\section{REFERENCES}

1. Russell R, Groves P, Taub N, O’Dowd J, Reynolds F. Assessing long- term backache after childbirth. Br Med J 1993;306:1299-303.

2. Butler R, Fuller J. Back pain following epidural anaesthesia in labour. Can J Anaesth 1998;45:724-8.

3. Macarthur A, Macarthur C, Weeks S. Epidural anaesthesia and low back pain after delivery. Br Med J 1995;311:1336-9.

4. Kock S, Hopf HB. Incidence and predisposing factors of persistent backache after lumbar catheter epidural anaesthesia in a nonobstetrical setting. Anasthesiol Intensivmed Notfallmed Schmerzther 1998;33:648-52.

5. Stevens RA, Urmey WF, Urquhart BL, Kao TC. Back pain after epidural anesthesia with chloroprocaine. Anesthesiology 1993; 78:492-7.

6. Collis RE, Harries SE. A subdural abscess and infected blood patch complicating regional analgesia for labour. Int J Obstet Anesth 2005;14:246-51.

7. Schmidt A, Nolte H. Subdural and epidural hematomas following epidural anaesthesia. Anesthesist 1992;41:276-84.

\section{G24}

\section{POPULATION-BASED SURVEY OF PAIN IN CHILDREN WITH SEVERE NEUROLOGICAL IMPAIRMENT}

\section{$\underline{\text { S Robertson }}{ }^{1}$, A Hunt ${ }^{2}$, K Seers ${ }^{3}$, A Thompson ${ }^{4}$}

${ }^{1}$ RCNI, Lichfield; ${ }^{2}$ University of Central Lancashire, Preston; ${ }^{3}$ RCNI, Oxford; ${ }^{4}$ North Warwickshire PCT, Nuneaton, United Kingdom

The Paediatric Pain Profile (PPP) is a twenty-item behaviour rating scale designed to assess pain in children with severe neurological impairments unable to self-report their pain. The current study assesses the usefulness, feasibility and acceptability of the PPP as a parent-held document.

AIMS: Prior to implementation of the PPP a regionally based pre-test survey was conducted to explore the extent to which parents report pain as a problem for children, and the effect pain has on the lives of children and parents. METHODS: A questionnaire was distributed to the parents of 125 children with severe to profound neurological impairments.

RESULTS: Currently, 47 parents have responded. Thirty-one $(67 \%)$ parents reported that their child had at least one pain. Twenty-five (53\%) described two pains, and thirteen $(27 \%)$ three pains. Similar to previous studies, the most commonly described sources of pain were muscle spasms, abdominal discomfort and headache. Current medication worked well for most children; however, it was unsatisfactory for $15 \%$. Twenty-seven parents $(57 \%)$ reported their child's pain caused them stress or worry and difficulty in sleeping. Twelve (25\%) parents reported their child's pain often or always interfered with the child's enjoyment of family activities. Eleven (24\%) children were reported to have pain every day or almost every day of the last four weeks and pain in twelve $(25 \%)$ was reported to be severe or very severe.

CONCLUSIONS: This work highlights the problem of pain for this group of children and the impact of pain on them and their parents. Pain is a cause of worry and concern for a significant proportion of families. Approximately $20 \%$ of children appear to have a major pain problem. We will assess whether the use of the PPP can assist in reducing the impact of pain on this population of children.
G25

SMART AND EASY TO USE “DISCOMFORT SCHNEIDER'S NONVERBAL TOOL" (DSNT)

I Ronen, L Kachko, R Efrat

Schneider Children's Medical Center of Israel, Petah Tikva, Israel INTRODUCTION: Pain assessment tools for nonverbal children are based on behavioural and physiological signs, which are not specifically related to pain experience. Most of the assessment tools are time consuming and has limited use on the busy hospital setting.

GOALS: Validity and reliability testing of the DSNT by other validated tools: FLACC and NIPS.

DSNT STRUCTURE: DSNT is a comprehensive, easy to use pain measuring tool for nonverbal children at all ages and health state. Five behavioural and three physiological signs are placed in three colored arrows. Every arrow refers to pain intensity level marked with numbers in ascending order. The first arrow represents the low pain level 0-3, the second arrow represents the medium pain level 4-6 and the third arrow represents the high pain level 7-10. According to the child's behavioural and physiological signs, the nurse selects one of the three available arrows. Then she scores a specific number from the relevant level depending on her perspective view of the patient.

METHODS: Two skilled nurses performed independent measurements simultaneously. 100 measurements of DSNT vs FLACC were performed in infants and toddlers. 228 measurements of DSNT vs NIPS (with and without physiological signs) were performed in premature and neonates. RESULTS: DSNT vs FLACC and vs the two versions of NIPS showed high correlation $(\mathrm{r}=0.8,0.89,0.86$ respectively; $\mathrm{P}<0.001)$ in measurements. Scoring of pain in patients with pain scores 1 and above were somewhat higher using DSNT comparing the NIPS.

CONCLUSIONS: The preliminary results show significant correlation of DSNT with FLACC and NIPS scores. The higher scoring with DSNT may represent the ability of the nurse to add her perspective view of the patient, which she cannot do with the NIPSS. The nurses easily adopted the DSNT and their compliance to pain assessment was remarkably elevated to $95-100 \%$.

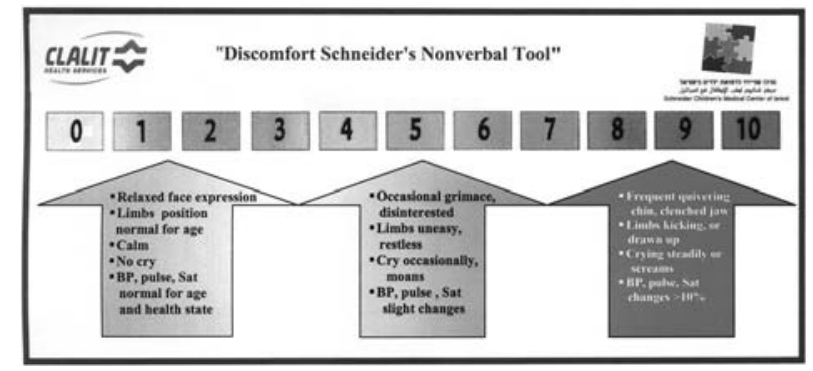

\section{G26}

CORRELATIONS BETWEEN VAS, FPS-R SCORES AND BEHAVIOURAL IMPAIRMENT IN CHILDREN SUFFERING OF ACUTE OTITIS OR PHARYNGITIS PAIN (GAVROCHE STUDY)

E Fournier-Charrière ${ }^{1}$, P Narcy ${ }^{2}$, P Reinert $^{3}$, G Olive ${ }^{4}$, F Allaert $^{5}$ ${ }^{1}$ Pain Unit, Bicêtre Hospital, Bicêtre; ${ }^{2}$ Robert Debré Hospital, Paris; ${ }^{3}$ Créteil Intercommunal Hospital, Créteil; ${ }^{4}$ Cochin University Teaching Hospital, Paris; ${ }^{5}$ Dijon Regional University Teaching Hospital, Dijon, France

BACKGROUND: Pain rating scales are rarely employed in ambulatory pediatric practice. In 2000, the French medical guidelines agency (ANAES) suggested to refer to "the basic activities of children, ie, moving, playing, sleeping, talking, eating", when pain scales are not available, but this method was not well validated.

METHODS: In the Gavroche study on acute otitis or pharyngitis pain, VAS (children $>5$ yo) or FPS-R scale (children $<5$ yo or their parents) were employed for all children (2733 otitis, 2710 pharyngitis) (see others abstracts by Narcy). Parents (2937) returned a questionnaire describing the course of pain during 3 days, including behavioural modifications of 
the child in 4 topics : sleeping, playing, eating, and the ability to go to crèche or to school, described by a 4 or 3 levels Likert-type scale (very impaired, moderately impaired, slightly impaired).

RESULTS: All the four items were strongly correlated with VAS scores given by the children $>5$ yo and with FPS-R scores given by children $<5$ yo or their parents (Anova test, $\mathrm{P}<0.0001$ at all levels for all the four items). CONCLUSION: In children suffering of acute otitis or pharyngitis pain, behavioural impairment in moving, playing, eating, sleeping is strongly correlated with pain assessment, either self assessment with VAS by children above 5 and FPS-R assesment by either children under 5 or parents. Therefore this method of assessing pain can be recommended to determine the level of pain and to choose the analgesics.

\section{G27}

\section{DEVELOPMENT OF A BEHAVIOURAL PAIN SCALE (EVENDOL) FOR CHILDREN UNDER 6 ATTENDING THE EMERGENCY DEPARTMENT}

E Fournier Charrière $^{1}$, F Lassauge ${ }^{2}$, C Ricard $^{3}$, B Tourniaire ${ }^{4}$, R Carbajal $^{5}$, B Falissard ${ }^{6}$, M Lemus ${ }^{7}$, P Cimerman ${ }^{5}$, P Turquin 7 , V Nouyrigat ${ }^{7}$, B Lombart $^{4}$

${ }^{1}$ Pain Unit, Bicêtre Hospital, Assistance Publique Hôpitaux de Paris, Le Kremlin Bicêtre; ${ }^{2}$ Saint Jacques Hospital, Besançon; ${ }^{3}$ Lapeyronie Hospital, Montpellier; ${ }^{4}$ Trousseau Hospital, AP-HP; ${ }^{5}$ CNRD, Trousseau Hospital, AP-HP; ${ }^{6}$ Paul Brousse Hospital, AP-HP, Villejuif; ${ }^{7}$ Bicêtre Hospital, AP-HP, Le Kremlin Bicêtre, France BACKGROUND: In the Pediatric Emergency Department (PED), it is mandatory to quickly assess pain before analgesic prescription. However, none of the existing pain scales is, at the same time, appropriate to all children under the age of self-assessment, to acute and prolonged pain, and to the emergency setting.

DEVELOPMENT OF THE SCALE: In 2003, five pediatric pain specialists and members of four PED staffs analyzed the items of all behavioural pain scales published either in English or French, and interviewed triage nurses to choose items that best reflected pain. The six selected items correspond to acute pain (complaints, consolability, grimace, movements) and to prolonged pain (postures, interaction with environment). Each item's description was largely discussed and tested with PED staff for comprehension. The scores of each item range from 0 to 3. The scale was tested at rest without any pain or anxiety-inducing stimulation, and during painful area examination.

Face validity

Different formats and wordings were proposed to about forty nurses. A definite format was chosen and shown again to nurses who found it clear and easy to use. These results confirmed good face validity.

Content validity

The analysis of the first 68 uses, showed that two-by-two correlations between items varied between 0.33 and 0.94 . Since the items consolability and interaction varied together ( $\mathrm{r}=0.85$ at rest, 0.94 at mobilization), they were put together in a single item in the final version of the scale. Therefore internal consistency in this first 68 uses of the tool appeared to be good.

CONCLUSION: The scale EVENDOL developed to assess pain in children in the emergency department seems to be simple to use and it showed good face validity and good content validity. The validation process goes on.
G28

VALIDATION OF EVENDOL, A BEHAVIOURAL PAIN SCALE FOR YOUNG CHILDREN ATTENDING THE ACCIDENT AND EMERGENCY DEPARTMENT

E Fournier Charrière ${ }^{1}$, F Reiter ${ }^{2}$, F Lassauge ${ }^{3}$, C Ricard $^{4}$,

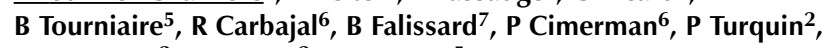
V Nouyrigat ${ }^{2}, C_{\text {Descot }}^{2}$, B Lombart ${ }^{5}$

1Pain Unit, Bicêtre Hospital, Assistance Publique Hôpitaux de Paris; ${ }^{2}$ Bicêtre Hospital, AP-HP, Le Kremlin Bicêtre; ${ }^{3}$ Saint Jacques Hospital, Besançon; ${ }^{4}$ Lapeyronie Hospital, Montpellier; ${ }^{5}$ Trousseau Hospital, AP-HP; 6CNRD, Trousseau Hospital, AP-HP, Paris; ${ }^{7}$ Paul Brousse Hospital, AP-HP, Villejuif, France

BACKGROUND: EVENDOL is a new scale developed to assess young children's (under 6) pain in the emergency department. It was elaborated by pediatric pain specialists and emergency staff members. Its face validity has been shown to be good. The scale includes 5 items, each scored from 0 to 3 . Total scores vary from 0 to 15 .

METHODOLOGY OF VALIDATION: The scale was tested first, at rest, without any painful or stressing stimulation (T1), and during mobilisation (T1bis), and then, after analgesic administration (T2, T2bis).

Various aspects of construct validity and interrater reliability were studied. Parent's consents were obtained. Children were assessed simultaneously by the triage nurse and one of the researchers (FR). Pain assessments by VAS were carried out by a nurse, one of the parents and the researcher. Pain was also assessed with other scales (CHEOPS, TPPPS, FLACC, EDIN) by the researcher. The anxiety and asthenia levels were assessed. A selfassessment score (FPS-R), was obtained from children above 4 yo. Thirty children were videotaped.

RESULTS: 297 children aged 1 month to 6 years were included.

Construct validity testing:

- Scores before and after nalbuphine: scores varied from 8.14 to 3.62 at rest $(\mathrm{P}<0.0001)$, and from 11.87 to 6.65 at mobilisation $(\mathrm{P}=0.0011)$, respectively.

- Correlations between VAS and EVENDOL scores as assessed by nurses and the researcher: correlations varied between 0.79 to 0.92 at all different times $(\mathrm{P}<0.0001)$

- Correlations between FPS-R and EVENDOL scores in 4-6 years old children: correlations varied between 0.34 to 0.64 .

Content validity and internal consistency were tested by determining the Cronbach coefficient which varied from 0.83 to 0.92 .

Interrater reliability was tested by comparing nurses' score and researcher's score: correlation varied from 0.87 to 0.98 .

CONCLUSION: This study validates EVENDOL, a new 5 items' scale to assess young children's pain in the emergency departments.

\section{G29}

\section{PAIN ASSESSMENT IN PROFOUND COGNITIVE IMPAIRED CHILDREN USING THE CHECKLIST PAIN BEHAVIOUR; IS ITEM REDUCTION VALID?}

LPeters $^{1}$, H Duivenvoorden ${ }^{2}$, M Dijk ${ }^{1}$, D Tibboel ${ }^{1}$

${ }^{1}$ Department of Pediatric Surgery; ${ }^{2}$ Department of Medical Psychology and Psychotherapy, Erasmus MC - Sophia, Rotterdam, The Netherlands

Children with profound cognitive impairment have commonalities and idiosyncratic features in their reaction to pain. It is unknown whether the variation in their reaction is greater than in any other population. To establish whether there are sensitive and specific cues of pain in this group of people we assessed whether the 23 item version of the Checklist Pain Behaviour could be reduced to ten items. After all, previous research demonstrated that only these 10 items discriminated between absence and presence of pain. Secondly, we wanted to explore the underlying structure of these ten selected items including its performance.

Data of 73 children were used. All these children were video-taped while they were admitted to the Sophia Children's Hospital for surgery, twice before and five times after surgery. These video-tapes were scored by independent observer. Presence of pain was assessed by a researcher using a visual analogue scale (VAS). We tested whether the underlying structure 
was unidimensional and whether it had differential qualities between pain and no pain, and to which degree. Using a modern psychometric method we unraveled the interdependency of the pain response in CI-children, in that the structure turned out to be unidimensional. In addition, these behaviours could be hierarchically ordered in terms of frequency of occurrences. Finally, these behaviours had to a high degree the potentialities to estimate the likelihood of occurrence of pain.

\section{G30}

CONSTRUCT VALIDITY, COMPLIANCE AND ACCEPTABILITY OF THE E-OUCH ELECTRONIC PAIN DIARY

LStinson $^{1,2}$, B Stevens ${ }^{1,2}$, B Feldman ${ }^{1,2}$, S Sidani ${ }^{2}$,

D Streiner ${ }^{2,3}$, G Petroz ${ }^{1,2}$, N Gill2, P McGrath ${ }^{4,5}$

${ }^{1}$ The Hospital for Sick Children; ${ }^{2}$ University of Toronto;

${ }^{3}$ Kunin-Lunenfeld Applied Research Unit Baycrest Centre for Geriatric Care, Toronto, Ontario; ${ }^{4}$ IWK; ${ }^{5}$ Dalhousie University, Halifax, Nova Scotia;

AIMS: The aims were to evaluate the construct validity and feasibility of measuring chronic pain in adolescents with arthritis using the e-Ouch electronic pain diary.

METHODS: A descriptive study design with repeated measures was used. A purposive sample of 76 adolescents aged 9-17 (M=13.5) with active arthritis was drawn from a rheumatology clinic in a university affiliated pediatric tertiary care centre. Participants were provided with a brief demonstration of the diary. Adolescents were signalled to complete the diary: (a) upon waking, (b) after school, and (c) before bed for a two week period. Construct validity was determined by comparing average weekly e-Ouch pain ratings with a priori predictions using the Recalled Pain Inventory, PedsQL Inventory and Arthritis Module, Pain Coping Questionnaire and disease activity indices. Adolescents completed an electronic questionnaire addressing the acceptability of the electronic diary.

RESULTS: As predicted correlations between average e-Ouch weekly pain intensity, unpleasantness and interference scores and scores from: (a) recalled average weekly ratings were positive in direction and moderate-high in magnitude $(0.45-0.84)$; (b) overall $(0.43-0.64)$ and disease-specific quality of life (0.46-0.58) scores and emotion focused pain coping $(0.18-0.50)$ were positive in direction, and low-moderate in magnitude; (c) and illness severity $(0.12-0.25)$ and number of active joints (0.01-0.06) was low. Overall compliance with the two-week study protocol was $77 \%$ (range $70-83 \%$ ). There was no significant differences in compliance rates between weekdays and weekends, sexes, younger or older children, or those with and without technical difficulties. However there was significant differences across time of day $(\mathrm{P}<0.008)$ and between weeks $(\mathrm{P}=0.0004)$. Adolescents rated the e-Ouch diary as highly acceptable and easy to learn, use and understand.

SIGNIFICANCE: Valid assessment of chronic pain could assist clinicians in tailoring interventions with the ultimate goal of accomplishing optimal pain management and enhancing clinical and developmental outcomes.

\section{G31}

\section{PRIMARY VALIDATION OF THE FRENCH VERSION OF THE POSTOPERATIVE PAIN MEASURE FOR PARENTS (PPMP)}

\section{Wood, C Cunin-Roy, A Pasturel, C Alberti, I Zaccaria, M Vieyra} Hôpital Robert Debré, Paris, France

The Post operative Pain Measure for Parents (PPMP) was developed to help parents to know when to medicate their child.

OBJECTIVE: To translate and validate the French version of the PPMP (originally consisted of 29 behavioural items (1)) by examining the relation between parent-report of child behaviours and child-rated pain, to create a pain assessment tool providing information to parents about when they should medicate their child.

METHODS: Participants were 68 children ( 30 boys, 38 girls) between ages of 7 to 12 and their parents.

Only children undergoing surgery known to cause at least a moderate amount of pain were included.
Children recorded:

1) their level of pain during the 2 days following surgery using the 6-point Faces Pain Scale, one of two randomly assigned period on each of the 2 days: between breakfast and lunch or supper and bedtime.

2) an emotional rating checklist (sad, angry, scared/afraid, nervous/worried). Parents completed the French version of the scale, during the 2 days following surgery.

Items reduction used clinical judgment, correlation between children and parents assessment of pain and the amount of missing data.

RESULTS: Among the 29 original items, 9 cues were selected that were predominantly linked with happiness, fear and anxiety felt by the children. A score was build varying between 0 and 24 with good discrimination (area under receiving operating curve of 0.80 ).

CONCLUSION: This study allowed us to simplify the French version to 9 items.

This shorter scale is to be tested and validated.

1) Chambers et al. Pain 1996;68:307-13.

\section{G32}

THE COMFORTneo FOR DAILY PAIN ASSESSMENT ON THE NICU

$\underline{M}$ van Dijk ${ }^{1}$, D Roofthooft ${ }^{2}, Y$ de Jager ${ }^{2}$, P van Deventer ${ }^{3}$, $\mathrm{G}$ van Blijderveen ${ }^{3}$, D Tibboel ${ }^{1}$

${ }^{1}$ Pediatric Surgery; ${ }^{2}$ Pediatrics, IC Neonatology, ${ }^{3}$ Erasmus MC Sophia, Rotterdam, The Netherlands

INTRODUCTION: The COMFORT behaviour scale was introduced in 1999 at our NICU during a study comparing morphine and a placebo (Simons et al, 2003). After completion of this study, pain assessment was neglected until a focus group took charge. First the COMFORT behaviour was adapted for use in premature neonates and introduced on the ward. In addition COMFORT cut-off scores were determined and a treatment algorithm developed for daily NICU practice.

METHODS: COMFORTneo was adapted by rewriting the item alertness, muscle tone and body movements and total score ranges from 6 to 30. The Visual Analogue Scale was replaced by a Numeric Rating Score (NRS) for both pain and distress. After a training session and ten test scores at bedside, interrater reliability of all nurses was calculated (Cohen's linearly weighted Kappa). From March 2004 until September 2005 COMFORT assessments were performed in between caregiving or acute procedures. Cut-off scores for the COMFORTneo were calculated using the Numeric rating scale (NRS) as a 'silver standard'.

RESULTS: Median Cohen's kappa of 80 nurses was 0.79 (range 0.65-0.94). 1164 COMFORT assessments were recorded in 209 infants. Median postnatal age was 3 days (IQR 1 to 11 ) and median gestational age was 30 (IQR 27 to 36 weeks). A NRS pain $>=4$ was seen in 206 out of $1103(18.7 \%)$ assessments and a NRS distress $>=4$ was seen in 245 out of $752(32.6 \%)$ assessments. Correlation between COMFORTneo and NRS pain and distress was 0.58 and 0.82 respectively. With cut-off scores set at 14 or higher for COMFORTneo, sensitivity was 0.80 and specificity 0.82 .

CONCLUSION: The derived cut-off scores may be used to develop a treatment algorithm. Scores of 14 or higher not always require analgesics or sedatives but may focus attention on comfort issues such as nonpharmacological treatment strategies.

\section{REFERENCES}

1. Simons SH, van Dijk M, van Lingen RA, et al. Routine morphine infusion in preterm newborns who received ventilatory support: A randomized controlled trial. JAMA 2003;290:2419-27. 
G33

\section{AGREEMENT BETWEEN CHILDREN AND PRIMARY CAREGIVER REPORTS OF CHILD PAIN AND SYMPTOMS IN PEDIATRIC PALLIATIVE CARE}

E Evan $^{1}$, J Hsiao ${ }^{1}$, M Wells ${ }^{1}$, J Saroyan ${ }^{2}$, M Byrne $^{3}$, M Erlich², W Schechter ${ }^{2}$, L Zeltzer ${ }^{1}$

${ }^{1}$ Department of Pediatrics, Pediatric Pain Program, UCLA Mattel

Children's Hospital, Los Angeles, California; ${ }^{2}$ Departments of Anesthesiology and Pediatrics, Columbia University Medical Center; ${ }^{3}$ School of Nursing, Columbia University, New York City, New York, USA

Children with life-limiting illnesses frequently experience pain and other symptoms which their primary caregivers report to health care providers. Though accurate pain and symptom reporting is crucial for good health care management, there are no published studies examining child/caregiver symptom communication in pediatric palliative care. This study hypothesized that (1) child/caregiver symptom ratings would be concordant, (2) caregivers would overestimate frequency, severity and distress of child symptoms, and (3) there would be higher concordance in child/caregiver ratings of observable symptoms (eg, vomiting) than in less observable symptoms (eg, nausea, anxiety). The Symptom Rating Scale (SRS) and the Memorial Symptom Assessment Scale (MSAS - Versions for 7-12 and 10-18 years old) were completed by 32 child/caregiver dyads (child mean age 14.5 years, range 9-21; 19 girls, 13 boys). MSAS scores demonstrated that child-caregiver concordance was high $(\mathrm{K}=0.75-0.57 ; \mathrm{P}<0.001)$ for appetite loss, nausea and tiredness, moderate $(\mathrm{K}=0.43-0.37 ; \mathrm{P}<0.05)$ for pain, itching, sadness and difficulty sleeping, and poor $(\mathrm{K}=0.06)$ for worry/anxiety. Total and subscale MSAS correlations were significant for older (10-18) but not for younger (7-12) child/caregiver dyads, as was pain frequency, severity and distress. SRS scores showed that child-caregiver agreement was highest $(\mathrm{r}=0.80-0.67 ; \mathrm{P}<0.001)$ for pain intensity and distress, difficulty sleeping intensity and distress, tiredness intensity, nausea intensity, appetite loss intensity, moderate $(\mathrm{r}=0.63-0.46 ; \mathrm{P}<0.05)$ for worry/anxiety distress, and poor $(r=0.45-0.04 ; \mathrm{P}>0.05)$ for sadness intensity and distress, worry/anxiety intensity, nausea distress, appetite loss distress. Caregivers overestimated child sadness $(\mathrm{t}=2.36 ; \mathrm{P}=0.027)$ on the MSAS-10-18, and child pain $(t=2.74 ; P=0.010)$, anxiety $(t=3.74 ; P=0.001)$, tiredness $(t=2.07 ; P=0.048)$ and sadness $(t=2.21 ; P=0.035)$ on the SRS. These initial results show that caregivers and children generally agree about the child's symptoms, though caregivers tend to overestimate symptom intensity and distress. Agreement was stronger for older children and for observable symptoms. The findings suggest that obtaining pain and symptom information from children as well as their caregivers will enhance care for children with life-limiting conditions.

\section{G34}

PEDIATRIC NURSES' SELF-REPORTED USE OF PAIN ASSESSMENT: A GAP BETWEEN EVIDENCE AND PRACTICE N Bandstra ${ }^{1}$, M McMurtry ${ }^{1}$, C Chambers ${ }^{1,2}$, C Court $^{3}$, C von Baeyer ${ }^{4}$

${ }^{1}$ Dalhousie University; ${ }^{2}$ IWK Health Centre, Halifax, Nova Scotia; ${ }^{3}$ BC Children's Hospital, Vancouver, British Columbia; ${ }^{4}$ University of Saskatchewan, Saskatoon, Saskatchewan

Adequate management of children's pain depends on accurate assessment. Despite growing evidence supporting the use of validated pain measures, little is known about actual clinical practice. We describe pediatric nurses' self-reported methods of pain assessment in a hospital setting. Participants were 197 pediatric nurses ( $98.5 \%$ female) recruited from inpatient and day surgery units (eg, Special Care Nursery, Emergency, Surgery, Intensive Care) who responded to a questionnaire directed to all nurses in a tertiary care children's hospital. Nurses reported how they typically assess pain in children. Trained raters coded the written responses into the following content categories: (a) behavioural cues (eg, crying, change in alertness, change in appetite), (b) physiological cues (eg, heart and respiration rate), (c) parent report, (d) child's verbal report, (e) patient history/past experience, $(\mathrm{f})$ validated behavioural measures and $(\mathrm{g})$ validated self-report

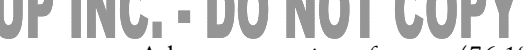

measures. A large proportion of nurses (76.1\%) reported using behavioural cues to assess pain, of which the most common were body language, facial expression, and change in emotional state. Nearly half of the nurses $(45.2 \%)$ reported that they used physiological cues to assess pain; the most common were observations of physiological distress, heart rate, and blood pressure. Few nurses reported using validated measures in assessing pain: only $21.3 \%$ reported using validated self-report measures (eg, FACES, Oucher, VAS) and only $7.6 \%$ reported using validated behavioural measures (eg, CHEOPS, NIPS). Logistic regressions were performed to identify demographic predictors of nurses' assessment strategies. The age group with which the nurse had the most experience was a significant predictor of assessment practice. The high self-reported reliance on physiological cues and the low use of validated self-report and behavioural measures suggest a gap between current knowledge regarding evidence-based pediatric pain assessment and clinical practice in hospitals. Future research should examine mechanisms for translating evidence-based approaches for pediatric pain assessment into practice.

\section{G35}

BARRIERS TO EFFECTIVE PEDIATRIC PAIN MANAGEMENT

M Czarnecki, T Hanson, C Armus, K Mohr, J Petrie, J Thompson, S Malin

Children's Hospital of Wisconsin, Milwaukee, Wisconsin, USA

INTRODUCTION: Children's Hospital of Wisconsin (CHW) has had a long-standing commitment to providing optimal pain management (PM). However, barriers continue to exist. Examples of barriers previously identified in the literature include inadequate physician orders, children's reluctance to report pain, parent's reluctance to have children receive medications, limitations in knowledge, and competing demands on nurses' times. The CHW Joint Clinical Practice Council (JCPC) queried nurses to identify perceived barriers at this institution.

METHODS: A survey developed by Vincent (2004) addressing potential barriers (eg, knowledge deficits; competing demands on RN's time; insufficient physician orders) was modified and distributed to nurses in all patient care settings. Nurses were asked to rank how much each barrier interfered with their ability to provide optimal pain management ( $0=$ not a barrier, $10=a$ major barrier). The study was approved by the IRB . ANALYSIS: Descriptive statistics including frequency distributions, means, and correlations were collected.

RESULTS: 272 surveys were returned. The 3 barriers with the highest mean (0-10) were a) inadequate/insufficient medical orders (4.95 \pm 2.60$)$, b) insufficient premedication orders prior to procedures $(4.92 \pm 2.81)$ c) inadequate time allowed to premedicate before procedures $(4.57 \pm 2.61)$. The 4 barriers with the lowest means included: a) limitations on my (the nurse) ability to assess pain $(1.34 \pm 1.51)$, b) low priority given to PM by nursing staff $(1.95 \pm 1.80)$, c) low priority given to PM by nursing management $(1.55 \pm 1.88)$ and d) low priority given to PM by myself (the nurse) $(0.53 \pm 0.955)$. Nurses identified feeling capable of overcoming the barriers they identified $(7.23 \pm 2.06)$ but also identified the potential of a great impact $(8.08 \pm 2.31)$ if they were systematically improved.

CONCLUSIONS: Results show that nurses perceive pain management as valued by themselves, their peers, and nursing management. Quality improvement efforts in the area of preprocedural communication and orders have the potential to significantly improve procedural pain management for children.

\section{G36}

CAN TEMPORARY TATTOOS IMPROVE CHILDREN'S PAIN ASSESSMENT?

$\underline{L}^{\text {Franck }}{ }^{1,2}$, K Oulton $^{2}$

${ }^{1}$ Institute of Child Health; ${ }^{2}$ Great Ormond Street Hospital, London, United Kingdom

BACKGROUND: Lack of availability at the point of care has been cited as a reason for infrequent use of pain scales. The current popularity among children of temporary tattoos led us to trial this technique to improve assessment and documentation of children's pain. We hypothesised that children with the temporary tattoos would feel more empowered to report 
their pain and that parents and nurses will more readily take action to treat children's pain.

METHODS: This RCT involved children aged 6-12 years who were admitted for planned inpatient (Group A, n=81) or day case (Group B, n=38) surgery. Prior to surgery, consent was obtained and children and parents were instructed in the use of the Wong-Baker Faces Pain Scale (WBFPS). Children randomly assigned to receive a temporary tattoo of the WBFPS (Groups A1 and B1) or to use the paper version (Groups A2 and B2). For inpatients, data were collected prospectively for 3 days about the child's pain assessment and analgesia. At discharge, parents and children completed a brief survey on the quality of pain care. For day case surgery, parents completed a simple 3-day pain management diary and pain care quality survey at home.

RESULTS: There were no differences in frequency of pain assessments, analgesia use or parent/child satisfaction between groups A1 and A2. However, children who received analgesia by continuous infusion or who had been seen by the Pain Service had more pain assessments and received more analgesia. On postoperative days 1 and 2 following day case surgery, group B2 performed more pain assessments. All children/parents in all groups reported they would use the same tool in future.

CONCLUSIONS: Temporary tattoos with pain scales were well received by children and parents. Further research is needed to understand the interaction of factors that influence frequency of pain assessment, analgesia and satisfaction with pain care for children.

\section{G37}

\section{SURVEY TO DETERMINE THE INCIDENCE OF WITHDRAWAL FOLLOWING PROLONGED TREATMENT WITH OPIOID AND BENZODIAZEPINE INFUSIONS IN CHILDREN}

IMcFadzean ${ }^{1}$, F Hutchison ${ }^{2}$, C Fabre1, M Sim ${ }^{1}$, L Buchanan ${ }^{1}$ ${ }^{1}$ Royal Hospital for Sick Children; ${ }^{2}$ University of Edinburgh, Edinburgh, Scotland

BACKGROUND: Continuous infusions of opioid drugs are commonly used for pain relief for surgical and medical conditions, and together with benzodiazepine infusions they are routinely used in the PICU. However, withdrawal symptoms have been reported with both groups when they are given by continuous infusion for five days. There is evidence to suggest that withdrawal symptoms may be prevented, or reduced, in some children by using a weaning plan. The purpose of this study is to describe the incidence, and prevalence of withdrawal symptoms in patients who receive opioid/benzodiazepine infusions.

METHODS: After ethical review, a prospective, descriptive and qualitative survey was undertaken, for a period of 2 months, on all patients who received opioid/benzodiazepine infusions for 3 or more days. Withdrawal symptoms measured included abdominal cramps, vomiting, diarrhea, tachycardia, movement disorder, mood changes, hallucinations and opioid withdrawal symptoms.

RESULTS: 25 patients, mean age of 2 years (0.2-13 years), weight $12 \mathrm{~kg}$ $(3-53 \mathrm{~kg}) .23 / 25(92 \%)$ were in the PICU and received both opioid and benzodiazepine (in the main, morphine and midazolam) infusions. The opioid mean number of infusion days was 5 (3-11 days), starting rate was $22(5-60 \mathrm{mcg} / \mathrm{kg} / \mathrm{hr})$ and maximum rate was $32(6-80 \mathrm{mcg} / \mathrm{kg} / \mathrm{hr})$. $3 / 25(12 \%)$ did not have any weaning measures, and $17 / 25(68 \%)$ had a documented weaning plan. $13 / 25(52 \%)$ had signs of withdrawal within 48 hours of discontinuing the infusions, and 9/25 (36\%) had three or more signs.

CONCLUSIONS: Symptoms and signs of withdrawal are poorly defined in the pediatric population. The symptoms are often vague and nonspecific. However, our small survey indicates that despite measuring pain and sedation daily in the PICU, and having weaning plans, there are a significant number of children who exhibit symptoms and signs of withdrawal. This is distressing for the child and the parent. Further detailed investigation is required to optimize prevention, treatment of the symptoms, and to quantify the consequences of this problem.
Pain in Developing Countries

\section{H1}

\section{SELF-PERCEIVED PAIN IN CHILDREN AND ADOLESCENTS ACCORDING TO GENDER - PREVALENCE, IMPACT ON DAILY LIVING AND ILLNESS-RELATED BEHAVIOUR}

A Roth-Isigkeit, A Ros, S Brueckner, W Baumeier, T Meier Department of Anesthesia, Luebeck, Germany

The aim of this study was (1) to investigate the prevalence of selfperceived pain in children and adolescents according to gender (2) its impact on activities of daily living and (3) illness-related behaviour due to pain in children and adolescents.

METHODS: Age-specific versions of the Luebeck Pain Screening Questionnaire were distributed to 11548 children and adolescents between the ages of 10-21 years. All pupils answered the questionnaire by themselves. 33 of 35 secondary schools in the district of Luebeck, Germany, participated in the survey. 9266 questionnaires were answered (return rate 80.2\%). 9148 of the 9266 (98.7\%) returned questionnaires were included in the evaluation.

RESULTS: $86.2 \%$ of the children and adolescents reported pain during the preceding three months (girls $90 \% *$ vs boys $82.6 \%$ ). Headache, abdominal pain, limb and back pain were the most frequent pain types reported. Girls reported more often than boys headache, abdominal pain and back pain. Boys reported more often limb pain than girls. The prevalence of pain with a duration longer than 6 months was $35.7 \%$ (girls $40.6 \% *$ vs boys $31 \%$ ). $33 \%$ of the children and adolescents reported three or more restrictions in daily living due to pain: $39.6 \%$ sleep problems (girls 48\%* vs boys 31.4\%), 31.6\% eating problems (girls $40.8 \% *$ vs boys $22.8 \%$ ), $27.2 \%$ missing school (girls $29.8 \% *$ vs boys $24.6 \%$ ), $34.2 \%$ inability to meet friends (girls $40.2 \% *$ vs boys $28.4 \%$ ), $44.6 \%$ inability to pursue hobbies (girls $47.7 \% *$ vs boys $41.6 \%$ ). $53.1 \%$ of children and adolescents reported doctor visits and/or use of pain medications due to pain (girls $59.5 \% *$ vs boys $46.9 \%$ ).

CONCLUSIONS: Girls reported more often pain than boys. Girls reported more often restrictions in daily living, doctor visits and use of pain medications due to pain complaints than boys. These results provide further evidence of the public health relevance of pain in children and adolescents. $* \mathrm{P}=0.000$

\section{$\mathrm{H} 2$}

\section{FAMILY CHARACTERISTICS OF CHILDREN WITH RAP AND HEADACHE}

M Mehta, M Sadhu

All India Institute of Medical Sciences, New Delhi, New Delhi, India

The influence of family characteristics and parent-child relationship on recurrent abdominal pain (RAP) and headache is controversial. In India, family bonding and relationships are very close-knit leading to both positive as well as negative influences on the pain behaviour of the child. We largely have extended families; about $30 \%$ of the families are joint in nature. The development of behaviour and personality is the result of interaction with multiple caretakers in a child's life.

The present investigation aims to make a comparative study of family characteristics of two common pain syndromes seen in child psychiatric clinic - RAP and headache. 50 children diagnosed as having RAP and 50 children having headache were included in the study. Both males and females in the age range of 8 to 14 years were included. Family Environment scale, Child Handling Questionnaire, Stressful life events scale and Semi-structured Performa were used for assessment.

The results indicate that children with RAP had mothers who often had abdominal pain. Pain behaviour was also present in other family members. In the headache group, high achievement orientation was seen on the family environment scale. There was less care and more control in both the groups. Significant stressful life events like chronic illness in the family, examinations, financial problems and death of grandparent were present in families of children with RAP and Headache. 


\section{H3}

\section{PEDIATRIC PAIN MANAGEMENT AFTER AN INCEPTION OF ACUTE PAIN SERVICE (APS) IN A UNIVERSITY HOSPITAL IN THAILAND}

W Krisanaprakornkit, D Horatanaruang, P Yimyaem, S Thienthong, R Sankotara

Khon Kaen University, Khon Kaen, Thailand

BACKGROUND: Even though pediatric pain is recognized as a major problem in health care worldwide, it may not be well recognized in some developing countries. In January 2004, Acute Pain Service (APS) was set up in Srinagarind Hospital; a university hospital in northeastern, Thailand. The service was run by department of Anesthesiology. The APS team composes of anesthesiologists and nurse anesthetists, who did a daily ward round for every case of patient receiving pain management initiated by anesthesiologist. This may have an impact on pediatric pain management. OBJECTIVE: To study the proportion and characteristics of pediatric pain service after an inception of APS

METHOD: We reviewed APS record of all pediatric patients from 1 January to 31 December 2004. Outcome measurement including age, sex, admission ward, pain type, pain management, pain assessment, satisfaction of pain management and need for the same treatment in the future were recorded.

RESULT: Service was rendered 265 times for 249 patients: 132 of whom were male $(53 \%)$. The children were 15 days to 15 years of age. Thirty-six, 34,26 and $3 \%$ of the service were provided for patients from orthopedic, pediatric, surgical and others (ENT \& gynecologic) wards respectively. Ninety-two and $8 \%$ of the service provided were for acute and chronic pain respectively. Opioid was the most common used drug $(83.4 \%)$. Continuous infusion was the most common used techique $(55.5 \%)$. Pain assessment was done and documented in $92 \%$ of patients. FLACC (41\%) was the most common used in young while NRS (30.2\%) was used in older children. In acute pain patient, patient or parental satisfaction was excellent in $91 \%$ of the service $(n=56)$ and all wish to receive the same technique in the future $(n=58)$. Comparing to the service before APS set up, we could provide more service for pediatric orthopedic patients (38.5\% vs $12.8 \%, \mathrm{P}<0.01)$.

\section{H4}

\section{BARRIERS TO EFFECTIVE PAIN MANAGEMENT IN A CAMBODIAN CHARITY HOSPITAL}

\section{K McNaughton}

McGill University School of Nursing, Montréal, Québec

This descriptive case study addresses barriers to pain management at a charity (nongovernment) hospital in Phnom Penh, Cambodia; one where over $50 \%$ of patients are children and the supply of opioids for pain management is limited. Specifically, the use of morphine in practice was assessed using a structured questionnaire with 10 items administered to all doctors $(n=6)$ working at the hospital during a one week period in July 2005. Reasons for morphine use as cited by doctors were: acid burns (4/6), amputations (3/6), osteotomies (2/6), bone grafts (2/6), and fracture reductions $(2 / 6)$. Four out of six doctors identified giving morphine as a routine part of the procedure (eg, amputation). Five of the six doctors reported that morphine was administered if the patient cried or if other pain medications had not worked. The use of a pain scale was cited by only one doctor. Problems perceived by doctors associated with increased morphine use at the hospital included patient addiction and side effects (5/6). The use of morphine in this Cambodian charity hospital by medical staff is different from western countries such as the US, Canada, or UK where guidelines for pain management for such procedures would include opioid analgesics and a reliable supply of opioids is available. Concerns about addiction to morphine and problematic side effects constrain Cambodian medical staff at this hospital from administering morphine to their patients for pain management.

\section{H5}

\section{A SURVEY OF PEDIATRIC PAIN IN THE KING CHULALONGKORN MEMORIAL HOSPITAL, BANGKOK, THAILAND}

$\underline{\text { S Niruthisard }}{ }^{1}$, P Seksarn ${ }^{2}$, D Bunchongsilp ${ }^{2}$

${ }^{1}$ Department of Anesthesiology; ${ }^{2}$ Department of Pediatrics, Faculty of Medicine, Chulalongkorn University, Bangkok, Thailand

BACKGROUND: University hospitals in Thailand still do not have established clear directions of pediatric pain management.

SETTING: Pediatric patients in the King Chulalongkorn Memorial Hospital (a university hospital in Bangkok) during September 15 to November 15, 2005.

DESIGN: A prospective, cross-sectional, descriptive study

OBJECTIVES: The primary objective was the result of implementation of pain management with selected tools for assessment of pediatric pain intensity. The secondary objectives were to collect the problems of the tools used, stimulate the recognition of pain assessment as the fifth vital sign for pediatric patients and locate the prototype place for further development in pediatric pain control.

METHODS: The pediatric patients were divided into 3 groups according to the age. The pain intensity of patients less than one year of age (group 1) were assessed by CRIES scale, between 1-5 years (group 2) by the Toddler/preschooler Postoperative Pain Tool (TPPS) and more than 5 years (group 3) by FACES Pain Scale or Visual Numeric Rating Scale (VNRS). The result of the pain assessment, management given and the attitude of the care providers towards the tools used were recorded.

RESULTS: The total prevalence of pain in children during the studied period of 2 months were 202 episodes which were $14.8 \%$ in group 1, $52.5 \%$ in group 2 , and $32.7 \%$ in group 3 . Most of the cases were noncancer patients $(71 \%)$. The pain score assessed were varied according to the pain problems. The responses provided for the pain problems were analgesics $(33.6 \%)$, nonpharmacological approach $(31.7 \%)$ and no treatment $(34.7 \%)$. The problem of the tool used was detected with CRIES resulting from the inconvenience to measure the noninvasive oxygen saturation. All the staff, residents and medical students caring the pediatric patients were encouraged to add pain assessment in routine clinical practice.

CONCLUSION: The selected tools according to the patient's age for assessment of pediatric pain intensity were practical with a minor problem. The result from this survey provided positive movement for further development of pediatric pain control which could be expanded to other hospitals in Thailand.

\section{H6}

\section{EFFICACY OF SINGLE HIGH DOSE PARACETAMOL IN PEDIATRIC OPHTHALMIC SURGERY}

\section{$\underline{\text { R Sunder }}$}

All India Institute of Medical Sciences, New Delhi, India

INTRODUCTION: Ophthalmic surgery is considered to be associated with mild to moderate intensity pain scores. Most of these surgeries are performed on day care basis and good postoperative pain relief hastens discharge times from the PACU [post anaesthesia care unit], reduces incidence of PONV [postoperative nausea and vomiting], increases early acceptance of oral feeds and reduces the usage of opiods.

Paracetamol is a safe and easily available drug in developing countries. There are some social taboos in our country to the use of this route by caregivers. There is no clear cut consensus on the appropriate dosing of rectal paracetamol [acetaminophen]. Various doses from $10-50 \mathrm{mg} / \mathrm{kg}$ have been reported in literature. Role of single dose paracetamol has also been debated.

MATERIALS AND METHODS: In our study, we enrolled children between 6 months-10 years undergoing ophthalmic surgery [lens aspiration, squint surgery, lacrimal duct surgery, trabeculectomy] after informed consent and education of the parents. 150 children were randomly allocated to three groups.Placebo group [P], High dose group [H],Low dose group[L].

All the subjects were induced using standard protocol [oxygen-nitrous oxide-sevoflurane/halothane], airway secured, intraoperative analgesia was provided by $2 \mathrm{mcg} / \mathrm{kg}$ of Fentanyl and paracetamol $20 \mathrm{mg} / \mathrm{kg}[\mathrm{L}]$ group or 


\section{COPYRIGHT PULSUS GROUP INC. " DO NOT COPY \\ J2}

$40 \mathrm{mg} / \mathrm{kg}$ in $\mathrm{H}$ group was administered rectally. The placebo group did not receive rectal paracetamol.

We studied intraoperative hemodynamics, intraoperative fentanyl requirements, Recovery score, OPS [Objective pain Score at 0 hrs, $30 \mathrm{mins}, 1 \mathrm{hr}$ and hourly thereafter for $8 \mathrm{hrs}$. Time to first demand of analgesic was noted. Rescue analgesia was provided by Fentanyl $1 \mathrm{mcg} / \mathrm{kg}$ ]. All children were advised oral ibuprofen at the end of the study period. PONV scores, total opioid requirement during the study period was also noted.

RESULTS: The $\mathrm{L}$ and $\mathrm{H}$ groups had similar recovery scores as $\mathrm{P}$ group.

The time to first analgesic demand was 55 minutes in $\mathrm{P}$ group, 145 minutes in $\mathrm{L}$ group, 350 minutes in $\mathrm{H}$ group.

The OPS scores were similiar at 0 and 30 minutes in all the three groups. The pain scores were significantly higher in the $\mathrm{P}$ group at 1 hour. The pain scores were higher at the end of 3 hours in the $\mathrm{L}$ group as compared to $\mathrm{H}$ group. PONV was lesser in the $\mathrm{H}$ group.

The results of this study will be presented in the conference.

\section{Standards, Guidelines, Ethics, Education}

\section{J1}

\section{A PROSPECTIVE EDUCATIONAL INTERVENTION ON PEDIATRIC PAIN MANAGEMENT FOR THIRD-YEAR MEDICAL STUDENTS}

I Cohen ${ }^{1}$, L Bennett ${ }^{2}$

${ }^{1} \mathrm{CNMC}$, Washington, District of Columbia; ${ }^{2}$ Northwestern University, Feinberg School of Medicine, Chicago, Illinois, USA INTRODUCTION: Over the last three decades, the misunderstanding and undertreatment of pediatric pain has been repeatedly documented. Unfortunately pain management receives little emphasis in medical education. Based on a needs analysis, a pediatric pain management intervention was incorporated into the Third-Year medical school curriculum. METHODS: To address areas of deficiencies: the development, assessment, and pharmacology of pain in children, a single-session problem-based discussion was designed by the pediatric and anesthesiology faculty. Students performed assessments using age appropriate pain scales and suggested treatment options. Pre- and postcourse knowledge were measured and a year-end assessment was performed. Attitudes and behaviours were reported using a 5-point Likert scale. Descriptive statistics, Student's t-test, and Yates-corrected Chi-square were used to analyze data. RESULTS: With Internal Review Board approval 127 students participated in the pediatric pain problem-based sessions. Post-test scores demonstrated statistically significant improvement in assessment and pharmacology. Comparison between students who had and had not participated in the intervention showed a statistical significant increase selfreported pain management activities.

DISCUSSION: After an educational intervention, third-year medicine students demonstrated a higher percentage of corrected answers regarding pediatric pain and reported increased pain management-related behaviours. To successfully address the persistent misunderstanding and undertreatment of pediatric pain, educators should seek different teaching and evaluation methods to accomplish learning objectives.

\section{Table 1}

Medical student pain-related attitudes and behaviours (Mean \pm SD)

\begin{tabular}{llll}
\hline Attitudes & $\mathbf{2 0 0 5}$ & $\mathbf{2 0 0 6}$ & P values \\
\hline $\begin{array}{l}\text { Appreciates importance of } \\
\quad \text { pain management }\end{array}$ & $1.4 \pm 0.5$ & $1.3 \pm 0.5$ & NS \\
$\begin{array}{l}\text { Comfortable assessing pediatric pain } \\
\text { Comfortable treating pediatric pain }\end{array}$ & $1.6 \pm 0.7$ & $1.3 \pm 0.6$ & $\mathrm{NS}$ \\
$\quad 2.9 \pm 1.1$ & $2.5 \pm 1.1$ & $\mathrm{NS}$ \\
$\quad$ behaviours & $3.7 \pm 1.1$ & $1.9 \pm 1.0$ & $<0.001$ \\
Assesses pain in children & $3.6 \pm 1.3$ & $2.4 \pm 1.1$ & $<0.001$ \\
Reports on pain in children & $4.0 \pm 1.0$ & $2.0 \pm 1.2$ & $<0.001$ \\
Checks analgesics orders and use & & &
\end{tabular}

\section{SEATTLE CHILDREN'S ANXIETY AND PAIN SCALE}

L Comden, D Ridling

Seattle Children's Hospital and Medical Center, Seattle,

Washington, USA

Comfort is defined as "to soothe in the time of affliction or distress - to ease physically or relieve" (www.dictionary.reference.com). To assure comfort in our patients, we must assess multidimensional components and need to individualize our treatment to each patient. The primary benefit of the SCAPS scale is that it allows the nurse to quickly assess the comfort level of the patient and in turn treat the patient accordingly.

We have found in our bedside practice that the experienced nurse often intuitively treats their patient for pain based on observation and assessment by being in tune with their patient. This scale is a method of validating this knowledge and documenting the assessment.

SCAPS - Seattle Children's Anxiety and Pain Scale

0 patient receiving neuromuscular blockade

1 no movement or response to stimuli, unconscious

2 very quiet, minimal movement, difficult to arouse

3 quiet, calm, arousable to speech or touch

4 cooperative with care, awake, cheerful, no complaints

5 restless, startles easily, consolable, furrowed brow

6 tears, moans, groans, whimpers, increasing complaints of pain with movement

7 crying, very fussy, difficult to calm, splinting, guarding, eyes tightly closed

8 anxious, agitated, grimacing, uncooperative with care, does not calm

9 extreme agitation, tense posturing or posturing or arching, not accepting mechanical ventilation, biting ET tube or breath holding 10 dangerously agitated, thrashing, climbing out of bed, pulling lines and tubes, unable to ventilate

We are presently in the process of completing our IRB application for a single-centre tertiary pediatric care facility, Seattle Children's Hospital and Medical Center, to test our interrater reliability and use this scale as our new standard of practice. We anticipate enrolling 120 patients ages $0-21$ from our three intensive care units. Research data shall be collected over a four-month period.

\section{J3}

\section{CHILDREN'S NURSES' PAIN MANAGEMENT PRACTICES: THEORETICAL KNOWLEDGE, PERCEIVED IMPORTANCE AND DECISION-MAKING}

\section{A Twycross}

Faculty of Health and Social Care Sciences, Kingston University St George's University of London, Streatham, United Kingdom BACKGROUND: Children continue to experience unrelieved moderate to severe pain postoperatively. Previous studies have suggested several factors to explain this. However, the impact of these factors on practice has not been explored. Nor have children's nurses' clinical decision-making strategies been examined.

AIMS: This paper will discuss a case study that set out to ascertain:

- How the perceived importance of pain management tasks impacted on practice?

- How theoretical knowledge impacted on practice?

- How what nurses said they did compared to what they actually did?

- How nurses make decisions when managing pain in children?

METHODOLOGY: Qualitative (participant observation and the think aloud technique) and quantitative methods (questionnaires) were used to obtain an in-depth picture of children's nurses' postoperative pain management practices. As well as examining some aspects of pain management for the first time, new perspectives were explored in relation to other well researched issues, including whether theoretical knowledge about pain management is applied in practice.

RESULTS: The perceived importance of a pain management task did not affect the likelihood of it being undertaken. A good level of theoretical knowledge appeared not to affect the quality of a nurse's pain management practices. A lack of congruence was found between what the nurses said 
they do and what the nurses actually did. Observational data indicated that nurses generally did not follow current recommendations fully when managing pain. Nurses appeared to use nonexpert decision-making strategies regardless of their years of experience or level of academic attainment. A hypothetico-deductive (analytical) model of decision-making seemed to be used.

DISCUSSION: For postoperative pain management practices to be effective, it appears that nurses need to have not only the right attitude and the right knowledge but also the ability to make the right decision. However, it is probable that other factors are also involved.

\section{J4}

\section{EDUCATION CHANGES MEXICAN NURSES' KNOWLEDGE AND ATTITUDES REGARDING PEDIATRIC PAIN}

\section{Huth, I Gregg, L Lin}

Cincinnati Children's Hospital Medical Center, Cincinnati, Ohio,

USA

This study explored the effectiveness of a pain education intervention on Mexican nurses' knowledge and attitudes toward pediatric pain. The framework that guided this study was based on the Theory of Planned Behavior (Ajzen, 1988). A convenience sample of 106 registered nurses from three hospitals in Mexico City was recruited. A Pediatric Pain Education Program (PPEP) was developed, implemented, and evaluated. The 3 hour program that was translated into Spanish consisted of pain assessment, physiology, and management; including pharmacological and nonpharmacological interventions. The effects of PPEP were measured in a one-group pretest-posttest design with a translated Spanish version of the Pediatric Nurses' Knowledge and Attitudes Survey (PNKAS) (Manworren, 2000). There were 79 nurses who completed both tests. A paired t-test indicated significant differences between pre and posttest results $(\mathrm{P}<0.0001)$ on the PNKAS. According to a repeated measure ANOVA, factors that had a significant effect on the change between pre and post-test responses were the hospital $(\mathrm{P}=0.0375)$ and years of nursing experience $(\mathrm{P}=0.0193)$. A paired $t$-test revealed no significant difference in terms of index of discrimination between the pre and posttests $(\mathrm{P}=0.1561)$. The pretest-posttest Kuder-Richardson-20 reliability coefficients were 0.65 and 0.72 , respectively. The intervention was effective in changing Mexican pediatric nurses' knowledge and attitudes. However, it was not known how long this effect was maintained. This study found that nurses who had more years of nursing experience had significantly more correct answers after PPEP. Health care professional's can share a common vision for pain management by increasing international collaborative efforts and by advancing pediatric pain knowledge.

\section{J5}

\section{ACADEMIC DETAILING FOR IMMUNIZATION PAIN REDUCTION: A PILOT STUDY}

N Schechter ${ }^{1}$, W Zempsky ${ }^{1}$, B Bernstein ${ }^{2,3}$, J Jones ${ }^{1}$, N Bright ${ }^{1}$ ${ }^{1}$ Connecticut Children's Medical Center, ${ }^{2}$ St Francis Hospital and Medical Center; ${ }^{3}$ University of Connecticut School of Medicine, Hartford, Connecticut, USA

INTRODUCTION: Studies suggest that traditional passive education (eg, lectures), has limited impact on changing clinician behaviour. "Academic detailing", in which tailored education is provided at the practice site, has had more success. We report on the pilot phase of an academic detailing project to address immunization pain, a common source of distress for children and families during their visits to the doctor. METHODS: Evidence-based approaches to immunization pain reduction were identified via consensus conference. Teaching materials including a video, poster, and parent brochure were developed. Four pediatric practices were selected for this pilot. Prior to educational intervention, telephone interviews were conducted with 115 consenting parents concerning recent immunization. Subsequently the study nurse provided inservice training reviewing immunization pain reduction. One month postpresentation, 68 additional parents were interviewed about their child's recent immunization experience.

RESULTS: In this pilot there is evidence of postintervention practice change. Parents were significantly more likely to have received written information about soothing their child $(\mathrm{P}<0.0001)$ and to have used comforting strategies $(\mathrm{P}<0.002)$ during the shot. An increase from 0 to $47 \%$ in sucrose/pacifier use for infants and evidence of a slight increase in parent satisfaction were also noted, but these were not significant with the pilot sample size.

DISCUSSION: In this pilot, a one hour teaching session in the office appears to have changed clinician immunization behaviour. Parents were far more likely to report that they had been given information about soothing or preparing their child for the shot, had learned new comfort measures and utilized them. Pacifier sucrose and overall satisfaction with injects appeared to increase as well. Based on these initial results a larger study of 30 additional practices for six months should ascertain how robust and long-lived these changes are.

\section{J6}

KID'S COMFORT: IMPROVING PROCEDURAL PAIN MANAGEMENT BY GETTING EVIDENCE INTO PRACTICE THROUGH ORGANIZATIONAL CHANGE - A BASELINE SURVEY OF PARENTS AND PATIENTS

$\underline{S \text { Penrose }}^{1}$, J Munro ${ }^{1}$, G Bowes ${ }^{2}$

${ }^{1}$ Royal Children's Hospital; ${ }^{2}$ University of Melbourne, Melbourne, Australia

The evidence reveals simple nonpharmacological and pharmacological, safe and cost-effective intervention can be used to minimize procedural pain and distress.

There are patient, clinician and systems barriers that have, to date, prevented the widespread adoption of some of these techniques.

AIM: To study parent and child experiences of care regarding procedural pain management, and suggestions for future practice improvements at a tertiary pediatric hospital. This was as a baseline prior to an organizationwide action research project and as part of a barriers to change analysis. METHODS: A questionnaire survey was developed, piloted and implemented over a 3 week period in a cross-sectional descriptive design. Data were collected from a convenience sample of patients and parents across 9 inpatient wards and 6 outpatient clinics. Information on patient demographics, past procedures and experiences of care. Questions using 3and 5-point Likert scales as well as qualitative responses were also used. RESULTS: 411 surveys (245 inpatients [IP] and 166 outpatients [OP]) across a variety of clinical areas. Most surveys were completed by the patient's mother. Almost all patients had experience with medical procedures $97 \%$ (IP)/81\% (OP). For planned admissions, $65 \%$ of parents were fearful their child would experience pain, only $37 \%$ of parents had anyone teach them how to help their child regarding pain or anxiety at procedures, and only $31 \%$ of patients had anyone teach them. $58 \%$ perceived their child had pain or anxiety at procedures during their admission, but $79 \%$ reported an intervention to decrease their child's pain. $64 \%$ of parents (IP) were present at their child's procedures. Data regarding perceptions about preparation, the procedure, restraint, and child coping will be presented. CONCLUSIONS: Parents and patients report a wide range of experiences associated with procedures at this pediatric hospital with many suggestions for improving care provision.

Acknowledgements: The National Institute of Clinical Studies.

\section{J7}

KID'S COMFORT: IMPROVING PROCEDURAL PAIN MANAGEMENT: GETTING EVIDENCE INTO PRACTICE THROUGH ORGANIZATIONAL CHANGE - A BASELINE SURVEY OF NURSING AND MEDICAL STAFF

S Penrose $^{1}$, IMunro ${ }^{1}$, G Bowes ${ }^{2}$

'Royal Children's Hospital; ${ }^{2}$ The University of Melbourne, Melbourne, Australia

The evidence reveals simple nonpharmacological and pharmacological, safe and cost-effective interventions can be used to minimize procedural pain and distress.

There are patient, clinician and systems barriers that have, to date, prevented the widespread adoption of some of these techniques. 
AIM: To study staff beliefs, perceived practice and self-described behav-
iours regarding procedural pain management, and suggestions for future practice improvements at a tertiary pediatric hospital. This was as a baseline prior to an organization-wide action research project and as part of a barriers-to-change analysis.

METHODS: A questionnaire survey was developed, piloted and implemented in cross-sectional descriptive design. Data were collected from a convenience sample of nurses and doctors working across 41 clinical areas at this tertiary pediatric hospital in Melbourne, For the week of survey distribution the number of potential respondents from the different professional groups was recorded. Data was analyzed as both a whole dataset, clinician subtype and by clinical area.

RESULTS: Surveys were completed by 206 nursing staff/145 medical staff. For nurses $78 \%$ were aged $<34$ years, $71 \%$ were employed for $<5$ years at the hospital and only $48 \%$ worked full time. $99 \%$ of nurses and $87 \%$ of doctors assist procedures. More than 35 different types of procedures with $>20$ different therapies used were reported. Nursing and medical staff varied in their experiences and level of training. Self-ranking of perceived level of competence in procedural pain management, current practice, attitudes and beliefs about pain management and barriers to change will be presented.

CONCLUSIONS: Staff varied in the education received or support available and the vast majority were interested in improving their skill base to better prevent and manage patient pain and distress. There is widespread support for interventions to improve how we manage procedural pain and distress at this institution.

Acknowledgements: The National Institute of Clinical Studies.

\section{J8}

\section{THERAPEUTIC MANAGEMENT AND OBJECTIVE}

EVALUATION OF PAIN IN CHILDREN CONSULTING FOR ACUTE PHARYNGEAL PAIN (GAVROCHE STUDY)

$\underline{\text { P Narcy }}^{1}$, P Reinert ${ }^{2}$, G Olive $^{3}$, F Allaert $^{4}$, E Fournier-Charrière ${ }^{5}$

${ }^{1}$ Robert Debré Hospital, Paris; ${ }^{2}$ Créteil Intercommunal Hospital,

Créteil; ${ }^{3}$ Cochin University Teaching Hospital, Paris; ${ }^{4}$ Dijon

Regional University Teaching Hospital, Dijon; ${ }^{5}$ Bicêtre University

Teaching Hospital, Le Kremlin Bicêtre, France

OBJECTIVE: To describe the course of the severity of acute pharyngeal pain using pain rating scales.

METHODS: Each physician had to enroll the first two children 1 to 12 years old, seen for acute pharyngeal pain and familiarize parents and/or children with the use of pain scales, to describe the course of pain during the 3 days following the consultation. Scales used were either a visual analogue scale ( 0 to 10 ) ( $\geq 5$ years old) or the Faces Pain Scale-Revised (0 to 10$)$ ( $<5$ years old). Assessment of course of pain was performed based on parents and/or children self-questionnaires.

RESULTS: 1445 physicians enrolled 2710 children of $6.3 \pm 2.9$ years old. Children had been suffering from a pharyngeal pain whose median severity was evaluated as $5.41+2.24$ for children $<5$ years old and $5.39+2.12$ for children $>5$ years old, despite prior self medication in $75.3 \%$ of them. Faced with such a pain, a level 1 analgesic compound was prescribed to $89 \%$ of children, a level 2 to $3.5 \%$ and a level 3 to $0.1 \%$. The following medications were also prescribed: antibiotic (39.5\%), NSAI $(29.5 \%)$, antipyretic drug $(31.2 \%)$, topical antiseptic $(28.7 \%)$, throat spray $(27.9 \%)$. Pain diminished by half on each day and disappeared almost completely on the third day.

CONCLUSION: Pain rating scales are performing tools to assess pain. Since almost $50 \%$ of children experienced a pain $>5$, this shows that selfmedication was ineffective and that pain was not adequately treated. These children should have been rapidly treated with a more effective analgesic treatment. In clinical practice, it is recommended to prescribe a progressive prescription, initiating treatment with level 1 analgesic and switching to an association NAIS/acetaminophen or level 2 in case of pain intensity $>5$ or nonresponse.
J9

\section{THERAPEUTIC MANAGEMENT AND OBJECTIVE \\ EVALUATION OF PAIN IN CHIDREN CONSULTING FOR ACUTE OTITIS MEDIA (GAVROCHE STUDY)}

P Narcy $^{1}$, P Reinert ${ }^{2}$, G Olive ${ }^{3}$, F Allaert ${ }^{4}$, E Fournier-Charrière ${ }^{5}$

${ }^{1}$ Robert Debré Hospital, Paris; ${ }^{2}$ Créteil Intercommunal Hospital,

Créteil; ${ }^{3}$ Cochin University Teaching Hospital, Paris;

${ }^{4}$ Dijon Regional Hospital, Dijon; ${ }^{5}$ Bicêtre University Teaching

Hospital, Le Kremlin Bicêtre, France

OBJECTIVE: To describe the course of the severity of pain in children with acute otitis media as rated on pain rating scales.

METHODS: Each physician had to enroll the first two children 1 to 12 years of age seen in a consultation for acute otitis media and familiarize parents and/or children with the use of either a visual analogue scale for children $>5$ years old or the Faces Pain Scale-Revised for children $<5$ years old, both of them rated from 0 "no pain" to 10 "intolerable pain". Assessment of course of pain during the 3 days following the consultation was performed on self-questionnaires returned directly.

RESULTS: 1445 physicians (88.7\% GPs and $11.3 \%$ pediatricians) enrolled 2733 children of $4.9 \pm 2.7$ years old. Mean pain severity was rated 6 despite prior self-medication in $82.8 \%$ of them. Faced with such a pain, a level 1 analgesic compound was prescribed in $87.9 \%$ of children, a level 2 to $5.2 \%$ and a level 3 compound to $0.2 \%$. The following medications were also prescribed: antibiotic $(74.6 \%)$, topical treatments $(31.8 \%)$, NSAI (30.5\%), antipyretic agent (23.0\%) and steroids (13.4\%). Pain diminished by half on each day and completely disappeared on the third day.

CONCLUSION: Pain rating scales are performing tools for proper management of pain, but require that a learning time be devoted to them. Prescription of a level 1 analgesic treatment for pain rated $\geq 6$ demonstrates that physicians underestimate such pain. In clinical practice, the ideal prescription would be a progressive prescription, starting with a level 1 analgesic compound, with a switch to an association NSAI/acetaminophen or a level 2 analgesic in case of pain intensity $>5$ or persistence pain $>3$ after 2 doses of analgesic.

\section{J10}

\section{PREHOSPITAL PAIN MANAGEMENT FOR CHILDREN: FOCUS GROUP REPORT OF EMS PROVIDERS}

M Kim $^{1}$, D Szewczuga ${ }^{2}$, D Simpson ${ }^{1}$, R Pirrallo ${ }^{1}$, H Hennes

${ }^{1}$ Medical College of Wisconsin; ${ }^{2}$ Milwaukee County EMS, Milwaukee, Wisconsin, USA

PURPOSE: Emergency medical service (EMS) professionals have identified relief of discomfort as the most relevant outcome measure. In designing an educational intervention to improve pediatric prehospital pain management, a focus group of EMS providers was used to obtain broad perspective and specific issues pertaining to pediatric pain management.

METHODS: Paramedics from our County EMS system were invited to participate on a voluntary basis. Our County EMS system is comprised of 19 municipalities serving nearly 1 million people. 234 paramedics from 13 departments provide advanced care including, by protocol, IV morphine for pain. This study was approved by our IRB. Consistent with focus group methodology, an interview protocol was developed with 10 key questions centred on pain (eg, assessment, factors associated with decisions to treat, attitudes associated with treatment, medical value of treatment) with targeted follow-up to explore the issues in greater depth and contextualized by presenting a child with a working diagnosis of an extremity fracture.

RESULTS: Nine providers ( 7 males, 2 females) with mean service years of 8.2 participated. Three were from urban and 6 from suburban departments. Providers often used verbal 0-10 scale as the faces pain scale was not useful, practical, and/or easily found on the "rig". The context in which the injury occurred (eg, mechanism, presence of mother/friends, language barriers), physical findings (eg, colour, pallor, breathing), prior professional and personal experience influenced their assessment and/or interpretation of child's pain. Distraction and RICE were commonly used, but pharmacological interventions were limited due to short transport times, belief that 
drugs would "mask" injury and delay treatment, and/or adverse reactions from ED personnel. Providers voiced limited knowledge and training in pathophysiology and effects of pain and desired information on outcomes of untreated pain.

CONCLUSION: EMS providers' assessment and management of pain in children is influenced by prior training, attitudes, and practicalities. To effect change, an educational intervention should emphasize use of appropriate evidence-based pain scales for children and proper use of pharmacological agents with pain pathophysiology, effects and outcomes of untreated pain as the foundational knowledge base guiding effective patient care.

(Supported by a grant from HRSA/EMSC \#H34-MC02548-01)

\section{J11}

\section{SPECIAL NEEDS REQUIRE SPECIAL ATTENTION: IMPLEMENTING THE PAEDIATRIC PAIN PROFILE (PPP) USING A PROJECT MANAGEMENT MODEL}

K Hunt $^{1}$, L Franck $^{2}$

${ }^{1}$ Great Ormond Street Hospital NHS Trust; ${ }^{2}$ Institute of Child

Health, London, United Kingdom

BACKGROUND: The project aimed to implement and evaluate the utility and acceptability of the Paediatric Pain Profile (PPP); a pain assessment tool for children with profound neurological impairment (PNI), who cannot communicate through speech (Hunt et al, 2004), in an inpatient setting.

SETTING AND SAMPLE: Five families on an orthopedic surgical ward participated alongside twenty nurses. Children were five to sixteen years of age with a primary diagnosis of cerebral palsy.

METHODS: The Classic Six Stage Project Management Model (Martin, 2002) was utilized. Data were collected through steering groups, education programs, parental interviews, nursing questionnaires, documentation audit and field notes.

RESULTS: Improvements and challenges were noted in three areas: nursing practices, individualized care, and parental partnership. Although nurses had identified the need for a new pain assessment tool for children with PNI there was resistance to change. Shared documentation was perceived as more time consuming. Nurses were found to need preparation and ongoing support to change their philosophy about pain assessment from a generic intensity score to a relational evaluation of individual behaviours. Individualisation of the PPP required preadmission assessment and parental involvement. Engagement between child, family and staff were improved in some cases, but numerous barriers were identified for full implementation of the PPP.

In conclusion, this project has shown that improvement of pain assessment for children with PNI requires a perceptual change from a discrete task to a pathway. Further exploration of implementation issues and evaluation of effectiveness will be required before the PPP can be more widely recommended for use in inpatient settings.

\section{REFERENCES}

Hunt A et al. Clinical Validation of the Paediatric Pain Profile. Developmental Medicine Child Neurology 2004;46:9-18.

Martin V. Managing projects in health and social care. London: Routledge, 2002.

\section{J12}

\section{FACTORS INFLUENCING NURSES' PAIN MANAGEMENT OF CHILDREN}

\section{Gimbler Berglund, K Enskär, G Ljusegren}

School of Health Science, Jönköping University, Jönköping, Sweden

Acute pain is still a problem with hospitalized children. The consequence of insufficient pain management causes unnecessary suffering for the children. Another consequence is that nurses feel frustrated and helpless while caring for children where pain management is difficult. The aim of this study was to identify factors that influenced nurse's pain management of children.

The subject consisted of 20 nurses working in one pediatric department at a middle sized hospital in Sweden. Interviews in accordance with the critical incident technique were used as a sampling method and the interviews were analyzed using content analysis. The result showed that there were thirteen factors divided into four different categories that influenced pain management. The categories were: Nurses, where the factors where: knowledge, experience, and attitude; Children, where the factors where: behaviour, diagnosis and age; Organization, where the factors where: prescriptions, routines, and support; and the fourth category was Nurse's cooperation with: doctors, children, and parents. The most frequent statements were made about the behaviour of children and cooperation with the doctors. If the children's behaviour were in congruency with the nurse's expectations, the nurses were more confident in the pain management, but if the behaviours of the children were confusing to the nurses then the pain management was difficult. When cooperation between nurses and doctors was good the outcome for the children was good pain management, but when cooperation between the nurses and the doctors was not good the children suffered more than necessary. This implies that the nurses need more knowledge of children's pain behaviour. The nurses need to use a variety of tools to interpret the behaviour of pain in children. Strategies to improve cooperation between nurses and doctors are needed.

\section{J13}

\section{AUDIT OF THE EFFICACY OF PAIN MANAGEMENT GUIDELINES IMPROVES ANALGESIA FOR CHILDREN}

$\underline{\text { P Brooks, }}$ H Pope, A Taylor, L Watson, T Kirwan

Chelsea and Westminster Hospital, London, United Kingdom

We present audit data to demonstrate that training of nurses and doctors to record pain scores and apply protocols improves the quality of pain management in children.

Following previous audit in 2003, the following action was taken:

1. Pain management protocols were revised by acute pain team and pharmacist.

2. Adolescent protocol added.

3. Modular training program introduced to ensure that every paediatric nurse has competency-based training in pain assessment and management. 4. Regular assessment of children's pain by nurses.

5. Analgesia given prior to painful procedures.

6. Regular analgesia offered to children expected to have pain.

METHOD: Pain was assessed by pediatric anesthetist in 200 consecutive medical/surgical patients. Nurse pain scores compared with patient report following direct questioning.

Incidence of moderate, severe and continuing pain and nausea and vomiting recorded.

2003 audit compared with that of 2005.

RESULTS:

\begin{tabular}{ccccccc} 
Year & $\begin{array}{c}\text { Pain score } \\
\text { recorded } \\
\text { in chart }\end{array}$ & $\begin{array}{c}\text { Pain } \\
\text { score }\end{array}$ & $\begin{array}{c}\text { Moderate } \\
\text { pain at } \\
\text { any time }\end{array}$ & $\begin{array}{c}\text { Severe } \\
\text { pain at } \\
\text { any time }\end{array}$ & $\begin{array}{c}\text { Pain } \\
\text { continuing } \\
\text { for }>\mathbf{4} \text { hours }\end{array}$ & $\begin{array}{c}\text { Nausea } \\
\text { or } \\
\text { vomiting }\end{array}$ \\
\hline 2003 & $27.5 \%$ & $73 \%$ & $17.5 \%$ & $4 \%$ & $7.5 \%$ & $10 \%$ \\
2005 & $93 \%$ & $91 \%$ & $10 \%$ & $2.5 \%$ & $2 \%$ & $8.5 \%$ \\
\hline
\end{tabular}

DISCUSSION: Regular assessment of pain experience improves quality of pain control.

Doctors and pharmacists provide the framework for good pain control, however acute pain nurse specialists ensure that the ward nurses have the skills to assess pain in order to follow the protocols.

Education of medical staff leads to better prescribing habits.

Adopting a multidisciplinary approach to pediatric pain management and acting on the results of audit allow pain management guidelines to be optimized. 
J14 COPYRIGHT PULSUS GROUP INC, DO NOT COPY

concerning pain in nursing records improved with $30 \%$. In $40 \%$ of medical records parent involvement in pain assessment and pain management was documented.

A NONSPECIALIZED TEACHING HOSPITAL: A TEN YEAR AUDIT CYCLE

\section{S Courtman}

Derriford Hospital, Plymouth, United Kingdom

AIMS: Using the audit cycle to demonstrate the demand for improvement in the management of pain in children and a survey on pain related clinical issues to demonstrate the need to improve education and support among staff working in acute areas of pediatric care, the demand for the creation of a children's pain service could be shown.

METHODS: All children who were inpatients during each study period were eligible to be included in the audit. The medical notes and prescription charts of each child were examined. Each child was interviewed using a structured questionnaire designed to assess current pain levels, pain management and side effects. Staff in all acute areas of pediatric care completed a simple questionnaire on pain assessment and management in children. RESULTS: Initial audit results (1996) demonstrated that only $16 \%$ of children with moderate-severe pain received appropriate analgesic intervention. Modification of the pain protocols resulted in an improvement in analgesic provision to $47 \%$ in the same group (2000). Repeat audit (2003) again showed some increase in analgesic provision but revealed only $1 \%$ had a documented pain score.

A survey of staff (2005) showed that $56 \%$ of staff found pain assessment confusing and although $96 \%$ were aware of assessment tools, only $51 \%$ believed it should be performed as a basic standard. $43 \%$ of staff were also not convinced of the benefits of regular prescribing of analgesics over PRN. 86\% believed a pediatric pain team would be of benefit.

CONCLUSIONS: Identification of the need to improve the provision of analgesia for children with moderate-severe pain and the need to improve the knowledge of attending staff have provided sufficient impetus for hospital management to recognise the need and approve the creation of a pain team for children.

\section{J15}

\section{DOES STAFF EDUCATION AND PREPRINTED TOOLS FOR NURSING RECORDS IMPROVE DOCUMENTATION AND PAIN MANAGEMENT IN CHILDREN?}

G Aagaard, B Simonsen, T Nielsen, S Jensen, V Storm, Y Dilling Copenhagen University Hospital Rigshospitalet, The Juliane Marie Centre, Copenhagen, Denmark

A general audit in accordance with the JCAHO, showed that in only $31 \%$ of cases, medical staff act according to the local guideline for pain assessment and documentation.

A study was undertaken to investigate pain management quality, including assessment, documentation and care in the postoperative period, as well as parent involvement.

METHODS: A qualitative study with data triangulation. The study period was four months in 2005 at the Clinic of Paediatric Surgery and Paediatric Cardiology. Data was collected from a multiple-choice test taken by nurses, questionnaires sent to parents and an audit of medical records.

INTERVENTION: Staff education and preprinted labels for nursing records concerning pain management. Staff were given pocket size VAS and FLACC scorecards for personal use.

RESULTS: Before the intervention $11 \%$ of nurses acted in accordance with local guidelines, after the intervention $75 \%$ did. Before the intervention $40 \%$ of nurses were able to account for the parameters in the FLACC scale, after the intervention $70 \%$ were.

Numerous parents experienced that they were informed on and involved in the management, but assessment and pain management depended entirely on the staff on duty and there was a lack of continuity in the treatment.

Medical records audit after intervention, demonstrate that use of VAS or FLACC noticeably improved. However, preoperative presentation of assessment tools for the children or parents was rarely documented. Children's coping strategies were described adequately. Documentation

This study demonstrates that education and improved documentation tools for nursing records improve documentation of pain assessment and management in children.

\section{$J 16$}

\section{THE DEVELOPMENT OF PAIN RESOURCE NURSES: IMPROVING PAIN MANAGEMENT AT THE BEDSIDE}

\section{$\underline{\text { L Palozzi, K Popovski }}$}

The Hospital for Sick Children, Toronto, Ontario

A single-day pain audit conducted at the Hospital for Sick Children in 2004 indicated areas for improvement in pain assessment and management practices. The hospital Pain and Sedation Committee used a variety of strategies to improve the use of evidence-based pain knowledge including dissemination of audit results, medical resident education and development of analgesia guidelines. The development of unit-based Pain Resource Nurses is presented as another method to disseminate current pain knowledge and implement practice change at the bedside and unit level. The advanced practice nurses in pain management developed a program to prepare bedside nurses to become unit-based experts in pain assessment and management. This poster will describe the process of developing and evaluating a Pain Resource Nurse program within a pediatric acute care academinc setting. Following REB approval, approximately 40 nurses will participate in an 8 hour educational program and complete the Pediatric Nurses' Knowledge and Attitude Survey about Pain (Manworren, 2002). The program will be followed up by monthly mentoring sessions facilitated by the advanced practice nurses which will provide ongoing support as nurses incorporate educational content into practice. Focus groups will be used to describe and evaluate the role at 6 months and 1 year following the educational program. Development and evaluation of the PRN role will be discussed. Implications for nursing practice, education and research will be highlighted.

\section{7}

\section{PROCEDURAL SEDATION RECORD: PROMOTING SAFE PRACTICE}

D Murray, L Palozzi

The Hospital for Sick Children, Toronto, Ontario

A hospital-wide procedural sedation policy was developed to promote safe and effective sedation practice at a pediatric academic health centre. The existing sedation record did not meet the published recommendations for the care of the pediatric patient receiving sedation. A new Procedural Sedation Record was developed by the Sedation Sub-Committee with representation from Anesthesia, Quality and Risk Management and pediatric sedation nursing experts. One year following the implementation of the record a hospital-wide audit was performed to determine utilization and knowledge gaps. Audit results indicated that while the record was used appropriately, lack of documentation in some areas indicated a need for further education and modification.

This poster will highlight the components of the procedural sedation record, audit results and implication for nursing practice and education.

\section{J18}

\section{PAIN AS THE 5TH VITAL SIGN: IT CAN BE DONE!}

LTyrrell, M Jones, C Menezes, E Petch, C Bouthillier, S Patterson The Hospital for Sick Children, Toronto, Ontario

OBJECTIVE: New advances in pain assessment and management of acute pain in children continue to evolve, yet a gap remains between research evidence and practice (Franck et al, 2000; Broome et al, 1996, Twycross, 1997). A multifaceted educational program was developed and implemented to integrate state-of-the-art pain assessment and management strategies into the clinical practice of pediatric nurses caring for children with complex medical conditions.

DESIGN: This poster will describe the program development process using Rush and Ogborne's (1991) Program Logic Model as a conceptual framework. 
It will further outline implementation strategies including literature review, nursing staff assessment questionnaires, design of innovative educational approaches, consultative and support processes, the creation of a culture for change, and collaboration with existing hospital resources such as the Acute Pain Service, Child Life Services, the Therapeutic Clown Program, and the Bravery Bead Program. Baseline data and one year outcomes will be presented, along with recommendations and suggestions for future research.

SETTING: The patient population served includes children from the divisions of Rheumatology, Nephrology, Gastroenterology, Hepatology and Nutrition, and Multi-organ Transplant, cared for in a pediatric tertiary care centre. The diversity of the patient population is well recognized yet repeated painful procedures, such as IV starts and surgical interventions, is a shared experience.

\section{REFERENCES}

Broome ME, Richtsmeier A, Maikler V, Alexander M. Pediatric Pain Practices: A National Survey of Health Professionals. Journal of Pain and Symptom Management 1996;11:312-20.

Franck L, Greenberg C, Stevens B. Pain Assessment in Infants and Children. Pediatr Clin North Am 2000;42:487-512.

Rush B, Ogborne A. Program Logic Models: Expanding their Role and Structure for Program Planning and Evaluation. Can J Program Eval 1991;6:96-106.

Twycross A. A Nurses' pesceptions of Pain in Children. Paediatric Nursing 1997;9:16-9.

\section{$J 19$}

\section{TEACHERS' PERSPECTIVES OF CHILDREN'S PAIN EXPERIENCES IN SCHOOL}

$\underline{\text { S Forsyth }^{1}, \text { L Hellsten }}{ }^{1}$, C von Baeyer ${ }^{2}$

${ }^{1}$ Department of Educational Psychology and Special Education;

${ }^{2}$ Departments of Psychology and Pediatrics, University of

Saskatchewan, Saskatoon, Saskatchewan

There is little research on teachers' experience with students in pain. To improve the management of children's pain at school, it is important to assess teachers' knowledge and concerns about child pain. The first phase of this research will examine the viewpoint of teachers with respect to children who experience pain in school (eg, children with recurrent, chronic, and injury-related pain). Specifically, (1) how do teachers respond to children in pain; (2) what information do teachers provide their students regarding pain; (3) what concerns do teachers have about students in pain; and (4) what information would teachers like to receive in order for them to work more effectively with children who experience pain.

METHODS: The data collection process will occur in three phases: two initial focus groups, a large distribution of questionnaires, and a small number of individual interviews. To gain a clear understanding of teachers' concerns in dealing with children who experience pain in school, informal focus groups will be conducted on the above questions with teachers in two elementary schools. Teachers' responses will be analyzed using qualitative methods and then applied in conjunction with a review of the literature to create a questionnaire to elicit information on teachers' views of students' pain experiences in school. Approximately 400 teacher questionnaires will be distributed to a random selection of Saskatchewan schools with the hope of obtaining at least 200 responses from teachers. Following item analysis and other statistical analyses of the questionnaire data, a selected subsample of the teachers will be interviewed using a semi-structured, informal process, to provide rich descriptions of the major themes arising from the questionnaire results. The results will be considered in light of existing research on health education and the prevention and management of pain in childhood.
J20

ACCREDITATION PAIN STANDARD: MAKING IT HAPPEN!

M Sawhney ${ }^{1}$, K Popovski ${ }^{2}$, J Stinson ${ }^{2}$, J Watt-Watson ${ }^{3}$, S Musclow ${ }^{4}$, C Kiteley ${ }^{5}$, K Brecht ${ }^{6}$, K Horrill ${ }^{7}$, N Thomson ${ }^{7}$, J Muir ${ }^{8}$, J Rae ${ }^{9}$, K Webber ${ }^{10}$

${ }^{1}$ North York General Hospital, ${ }^{2}$ The Hospital for Sick Children; ${ }^{3}$ University of Toronto, Faculty of Nursing, ${ }^{4}$ The Scarborough Hospital, Toronto, ${ }^{5}$ Credit Valley Hospital, Mississauga, Ontario; ${ }^{6}$ Montreal General Hospital, Montréal, Québec; ${ }^{7}$ Purdue Pharma Canada, Pickering, Ontario; ${ }^{8}$ Providence Health Care, St Paul's Hospital, Vancouver, British Columbia; ${ }^{9}$ Foothills Medical Centre, Calgary, Alberta; ${ }^{10}$ Memorial University of Newfoundland, School of Nursing, St John's, Newfoundland

The Canadian Council on Health Services Accreditation (CCHSA) has included pain assessment and management in its new CCHSA Standards published in 2005. These standards guide the accreditation process for health care organizations across Canada and specific actions are suggested that relate to pain assessment, management, monitoring, organizational responsibility, and assessment measures. The standard is evidence-based and includes the organization's accountability to train and update staff, patients and families on pain management options and strategies. To facilitate the implementation of these standards with children and with adults, a guide has been developed outlining strategies and related rationales that professionals can use to meet the new pain assessment and management requirement from the CCHSA. This guide was prepared as a resource by the Canadian Pain Society - Special Interest Group on Nursing Issues, and has been reviewed by an interprofessional group of pain experts.

The purpose of this poster is to highlight the key components of the guide that were developed to assist organizations in meeting the pain management standard. Specific strategies and tactics that organizations can utilize in meeting and maintaining the CCHSA standard will be presented. 


\section{A}

Aagaard G .............J15

Abbas A .................. 35

Aburto $\mathrm{F}$..............E34

Adriana $\mathrm{V} \ldots \ldots \ldots \ldots \ldots$. . E35

Ahlqvist J ...........C17,C20

Ahola S .........E28,E33

Aigrain $Y \ldots \ldots \ldots \ldots \ldots . C 28$

Alberti C ..............G31

Albertyn R ...........2.101.1

Aldridge L . . . . . . . . . . . C41

Alexander S .............C36

Alibeu A ...............C27

Alijani $\mathrm{H} \ldots \ldots \ldots \ldots \ldots$. $\ldots$ 1

Alijany $\mathrm{H} \ldots \ldots \ldots \ldots \ldots$. . C2

Allaert F ….....G26,J8,J9

Amrutia D ............. D18

Amtmann D ............G11

Anand K . . . 4.301,4.301.2,A1,A2

Anand KJS . . . . . 2.201,3.301.1

Anand S . . . . . . . . . . . . 2.401

Anderson BJ . . . . . 2. 2.401,2.401.3,

$.2 .601 .2,3.301 .2$

Anderson C .............F13

Anderson Khan K .....E21,E22

Anne Claire D ..........G18

Anne T . . . . ..........G18

Annequin D .....E23E29,E34,

G18,G21,D14

Anthony K . . . . . . ....E25

Aranda JV . . . . . . . 2.201,B5,E29

Armus C ...............635

Autret-Leca E . . . . . . . . .F4

Aynsley-Green A . . . . . . . . B17

\section{B}

Babl F . . . . . . . . . . . . 4.402.2

Bagry $\mathrm{H} \ldots \ldots \ldots \ldots$. . . . . 23

Ballard R ............G11

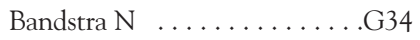

Bao V ................E29

Baos J .............E19,G19

Bartocci M ...............B7

Baumeier W ............H1

Bazan C ...............E29

Bean-Lijewski J . . . . . . . . D13

Beattie T . . ...........C41

Bell L . . . . . . . . B B1,B2

Bennett L . . . . . . . . . . .J1

Bennett $S \ldots \ldots \ldots$. . . . . . . 66

Benoit M ........E24,E26

Berde C . . . . . . . . 2.404,3.203

Berg M ............G20

Berg S . . . . . . ........ 229

Berlin $\mathrm{K} \ldots \ldots \ldots \ldots$. . E5

Bernstein B ............J5

Besenski L .............G9

Beyene J . . . . . . . . . B14,B15,B16

Beyer JE . . . . . . 2.505,2.505.1

Bhat A ............... D6

Bhatia A .............. D6

Biraud J ............... 228

Black S . . . . . . . . . . 4.302

Blass E . . . . . . . . . 3.202,3.202.3
Blount R ...3.201,3.201.2,3.204, 3.204.2,3.204.3,E24,E26

Boelen-Van Der Loo T . . . . . . B19

Bösenberg AT . . . . .5.101.2,F12

Bouthillier C ..........J18

Bowes $G \ldots \ldots \ldots \ldots . . \ldots 6,57$

Brake L . . . . . . . . . . C32

Bratt E . . . . . . . . . . . C33

Breau L . . . . . . . . . . . . . . B15

Brecht K ............J20

Bricher G . ...........D17

Bright $\mathrm{N} \ldots \ldots \ldots \ldots \ldots . \ldots \ldots$

Brodecki D ...........G17

Brooks P .............J13

Brouillard J . . . . . . . . . .G23

Brown A .............................

Brown $\mathrm{K} \ldots \ldots \ldots \ldots$. . . . . . . 23

Brown $\mathrm{S} \ldots \ldots \ldots \ldots$ A3,E9

Bruce E . . . . . . . . . . . . . . . . . . . .

Brueckner S ...........H1

Brun $S$................E13

Bruni O . . . . ............2.205

Brunsson I . . . . . . . . . . C29

Buchanan L . . . . . . C41,G37

Bunchongsilp D .........H5

Byrne $M$..............333

\section{C}

Caldwell-Andrews A $3.204,3.204 .2$

Camfield C . . . . . . . . . . . . . B16

Campbell R .........E24,E26

Campbell-Yeo M .........B1,B2

Carbajal D ............E34

Carbajal R ...2.201,3.202,3.202.2,

..........E29,E34,G27,G28

Carollo J .............G10

Carroll B ..............G14

Carter B ................. B13

Casale P . . . ............C43

Cassidy B ..............D17

Castillo W .............E34

Chalut D . . . ..........C22

Chambers CT …2.205,2.505.2, . . . . . . . . 2.505,5.101.1,

.......C30,D2,E18,G12,G34

Champion $G \quad \ldots \ldots \ldots \ldots$. F5

Charette S ............C18

Charlesworth A ..........F4

Chaumont B ...........C28

Chen E ...............E18

Chen J . . . ............... F13

Cheng P ......... 3.204,3.204.3

Chimello J ..............B11

Choinière $\mathrm{M} \ldots \ldots \ldots \ldots$ C22

Cignacco E . . . . . . . . . . B12

Cimerman P . . . E34,G27,G28

Clancy C . . . . . . ......D3,D4

Clare H ................. 41

Clarke H .............F4

Clemente I . . . . 2.402.1,4.205.1,

.............5.204,5.204.1

Clinch J . . . . . . . . . . . . .G5

Coates C ..............D15

Cofer B .................
Cohen I . . . . . . . . . . .G2,J1

Cohen LL . . . . 2.402.2,2.501.2,E2

Coleman C . . . . . . 4.303.3,G17

Collins JJ . . . . . . 4.401.3,4.401.2

Combs S ...................

Comden L . . . . . . . . . . . . . J2

Connell H . . . . . . . . . .G5

Connelly M ........E25,E32

Corrêa Castral T . .......B21,B22

Coulet C ..............B2

Court C .........................

Courtman S ...........J14

Cox D .................66

Craig K . . . . . . . 2.204.3,D2

Craigie M . . . . . . . . . . . . . . C26

Crandall M . . . . . . 2.503,2.503.2

Cucchiaro G ........C38,C43

Cugler T . . . . . . . . . . . . B11

Culbert T . . . . . .........C3

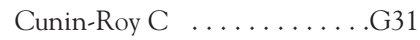

Cuy R ................ 38

Cyrenne L . . . . . . . . . . C18

Czarnecki .....3.203,C42,G35

\section{D}

Dampier C ...................... Damschen U ............... 19

Darlington A . . . . . . . . . E8

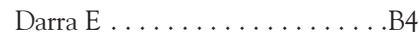

Davies H ...........C42

Davies W ...E4,E5,E21,E22,E30

Davis K ............... 3

De Crouy A ............E23

de Graaf J .............E20

de Jager $Y \ldots \ldots \ldots \ldots$. . . . . . . . . . . .

de Liefde I $\ldots \ldots \ldots \ldots \ldots$. . . . .

Descot C . . . . . . . . . . . G28

Deshpande J .........B13,C14

Devine K . 3.204,3.204.3,E24,E26

Dholakia K .............C32

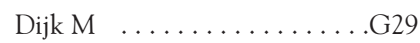

Dilling Y .............J15

Dimmagio T . . . . . . . . . . C38

Din L ...........E28,E33

Dixon K . . . . . . . . . . . . . C3

Dmytriiev D . . . . . . . . . . . D1

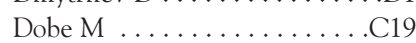

Doctor T ............. D18

Dolan L ............... . . . . . .

Dooley K ..........E24,E26

Dowden S . . . . . . . . . 4.402.2

Drummond-Lewis J . . . . . . . C40

Duivenvoorden H . . . A1,A2,G29

Duparc N ................. 28

Dupuis A ..............B15

\section{E}

Eakins D ...........E7 Eccleston C ......4.403,4.403.1, ........4.403.2,G4,G5

Edith $G \ldots \ldots \ldots \ldots \ldots$ G18

Edwards C ............E25

Efrat R .............. $23, \mathrm{G} 25$

Ely B . . . . . . 4.303.1,4.303.3,G17
Engel J . . . . .......E3,G11

Enskär K ............J12

Erlich M ............G33

Evan E ..........4.204.2,G33

Evrot $\mathrm{S} \ldots \ldots \ldots \ldots \ldots \ldots$

\section{$\mathbf{F}$}

Fabre $\mathrm{C} \ldots \ldots \ldots \ldots$. . . . 337

Falissard B ......G27,G28

Fayeni $\mathrm{P} \ldots \ldots \ldots \ldots \ldots$. . E1

Feinstein A . .........G22

Feldman B ...........G30

Fellman $\mathrm{V} \ldots \ldots \ldots \ldots$. . . B23

Fernandes A .........E27

Ferrise A .C42,E4,E5,E21,E22,E30

Figueroa E ..........E34

Filion F ............B

Finkelstein M ...........C3

Finley A . . . . . . B1,B2,B16

Finnström B ........C6,F11

Fiquet $\mathrm{S} \ldots \ldots \ldots \ldots \ldots \ldots$ C28

Fitzgerald M .......2.601.1,C3

Fitzpatrick E . . . . . . . . . . C30

Flores M ............E35

Flores Muñoz MA . . . . . . . . 2.202.1

Fonareva I . . . . . . . . . G15

Forgeron $\mathrm{P} \ldots \ldots \ldots \ldots \ldots$. . E11

Forin $\mathrm{V} \ldots \ldots \ldots \ldots \ldots$ D14

Forsyth $S \ldots \ldots \ldots \ldots$ J19

Foster R ............G10

Fournier Charrière E ...G27,G26,

..................... 28 . J

Fournier M . . . . . . . . . . C28

Fournier-Charriere F . . . . . C27

Frager $\mathrm{G} \ldots \ldots \ldots \ldots$. E6

Franck L . . . . . . . 5.202,5.202.1, .......B15,B16,B17,G36,J11

Franck LS . . . . . . . . . 4.402.1

Franks S . . . . . . . . 4.203.1

Friedrichsdorf S . . . 4.401.3,E13

Frigon C . . .............. 14

Fulton I $\ldots \ldots \ldots \ldots \ldots \ldots$ C26

\section{G}

Galante D . . ...........B3,D5

Gallo M ................ 34

Ganesh A . . . . . . . . . . C38,C43

Garske D ............E13

Gaspardo C ......................

Gatbois E ..............E23

Gedaly-Duff V ...........F10

Ghayem Hasankhani E . . . . . . G1

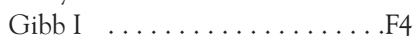

Gibbins S .................. B16

Gill N ..............G30

Gilleland J .........E24,E26

Gimbler Berglund I .......J12

Giordano L . . . . . . . . . C32

Goddard J . . . . . . . . . . . C35

Gold J . . . . . . . . . C8,E7

Goldbloom R . . . . . . . . . D15

Goldschneider K . . . . . . . 4.302

Gomez J . . . . . . . . . . . . .E34

Goubert L . . 3.201,3.201.3,5.203.1 
Goulet C $\ldots \ldots \ldots \ldots \ldots \ldots$. $\ldots 28$

Greenberg $\mathrm{S} \ldots \ldots \ldots \ldots \ldots \ldots \ldots \ldots \ldots \ldots$
Gregg $\mathrm{T} \ldots \ldots \ldots \ldots \ldots \ldots$

Grégoire M ...........E6

Grégoire M-C . . . . . . . . . 5.204,

.............204.1,5.204.2

Grey M ......E14,F6,G13

Griffin J . . . . . . . . . . . .C9

Grunau R ................66

Grunau RE . . . . . . 4.101.1,G21

Guite JW . . . . 5.201,5.201.2,F3

\section{H}

Häfner R ............. 24

Haig M . . . . . . . . . . . . C18

Hails S . . . . . . . . . . D8

Hains A .....E4,E5,E21,E22,E30

Hainsworth K .E4,E5,E21,E22,E30

Hamelin V . . . . . . . . . . C28

Hamers J . . . . . . . . . . . B12

Hammond C . . . . . . . . . . G9

Hancock R . . . . . . . . . . . . B8

Hanrahan K . . . . 2.504.1,2.504.2,

.............2.504.3,C11

Hanson T . . . . . . . . . . G35

Harrison D ............................

Hart $\mathrm{T} \ldots \ldots \ldots \ldots \ldots$. E9

Hatchette J . . . . . . . . . .5.201

Hays $S \ldots \ldots \ldots$. . . . . . . . 13

Hellsten L . . . . . . . . . . .J19

Hellström-Westas L . . . . . . . B23

Henkel W ............E13

Hennes $\mathrm{H}$. . . . . . . . . . . . J10

Hermann C . . . . . 2.403.2,D9, D10

Heron B ............G21

Hesselgrave J ..........G7

Ho $G \ldots \ldots \ldots \ldots$. . . . . . . 6

Hockenberry M .........G7

Hodge D . . . . . . . . . . . . . B13

Hofman A .............. E8

Högberg $\mathrm{L} \quad \ldots \ldots \ldots \ldots \ldots$ F7

Hohmeister J . . . . . . . . D9, D10

Holdridge-Zeuner D .......C15

Holmberg T . . . . .......... 29

Hoogstraten J . . . . . . . . . . G8

Horatanaruang D .........H3

Horrill K ...............J20

Hotopf M . . . . . . . . . 4.101.2

Howard RF .......4.402.1,B17

Howlett A . . . . . . . . . B15,B16

Hsiao J . . . . . . . . . . . . . G33

Hubert I . . . . . . . . . . . . C22

Hughes A ..............E6

Huguet A . . . . . . . E19, G19

Hunfeld J . . . . . . . . . . . . E8

Hunfeld JAM . . . . . . . . 5.201.1

Hunt A ................ G24

Hunt $\mathrm{K} \ldots \ldots \ldots \ldots \ldots \ldots \ldots 11$

Hunter E ........................

Hutchison $F \quad \ldots . \ldots \ldots . . .637$

Huth M .............J4

\section{I}

Ipp M . . . . . . . . . . . D16 Ipsiroglu $\mathrm{O} \ldots \ldots \ldots \ldots .2 .205$

Isaac $L \ldots \ldots \ldots \ldots \ldots \ldots$. C39

Jacob E ......2.503,2.503.1,G7

Jaddoe $\mathrm{V} \ldots \ldots \ldots \ldots$. . . . . . . . . .

Jahng $\mathrm{S} \ldots \ldots \ldots \ldots \ldots$ C5

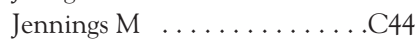

Jensen $M \ldots \ldots \ldots \ldots \ldots$. E3

Jensen $\mathrm{S} \ldots \ldots \ldots \ldots \ldots \ldots$ J15

Johansson M ..........F9

Johnson A ............F10

Johnson $G \quad \ldots \ldots \ldots$ E24,E26

Johnson K ............F10

Johnston C . . . . . 3. 3.202,3.202.1,

$\ldots \ldots \ldots \ldots$ B1,B2,B16,C22

Johnston L . . . . . . . B B, B10

Johnston S . . . . . 4.301,4.301.3

Jones $G \quad \ldots \ldots \ldots \ldots \ldots$. E12

Jones J ................ J5

Jones M ...........J18

Jordan A . . .2.503,5.203.3,G4,G5

Joseph M ............. 8

\section{K}

Kachko L ........C23,G25

Käck B ................C6

Kaczka C ............C31

Kain Z .3.204,3.204.2,E14,F6,G13

Kankkunen P . . . . . . . . .E14

Kant A . . . . . . . . . . C8,E7

Katz J .........B4,B14,C23,E9

Kauffman R ...........D13

Kennedy E . . . . . . . . . . 4.303.1

Khan K . . . . 4.201,E4,E5,E30,F2

Kikhahi B ............C1

Kim S . . . . . . . . . . C8

Kirwan $\mathrm{T} \ldots \ldots \ldots \ldots \ldots$ J13

Kiteley C ...........J20

Kleese A ............G20

Kleiber C .........................

Klein V . . . . . . . . . . . . . . B11

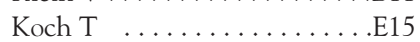

Kogan C ...............

Koh J ...........D13,G15

Kokinsky E . . . . . . . . C33,F9,F11

Kokki H .............E14

Kornegård-Bark § . . . . . . . . . C33

Kraft N . . . . . . . . . . . . . B13

Kreicbergs U .......... 4.204.3

Krisanaprakornkit W $\quad .2 .202 .2, \mathrm{H} 3$

Kristjansdottir $\mathrm{O} \ldots \ldots \ldots$. . . . E31

Kudirka D ............C22

Kuiters S . . . .........E8

Kuttner L . . . . . . . . . . 4.304.2

Lacoste Munoz P . . .......G18

Ladwig R …....4.201,E4,E5,

............E21,E22,E30

LaFonde D . . . . . . . . . . G2

Lamont J . . . . . . . . . . .F5

Lander J ............... B20

Lange BC .......4.202.1,C26

Lanier B . . . . . . . . . . . .C15

Larochette A ............ D2

Larsson B . . . . . . . . . . 5.201

Lassauge F . . . . . G27,G28

Lassauge $\mathrm{L} \ldots \ldots \ldots \ldots \ldots$. C27

Lassere M .............F5
Lavella H ........... . 4.303.1

Le May $\mathrm{S} \ldots \ldots \ldots \ldots \ldots$ C22

Leahy $S \ldots \ldots \ldots \ldots \ldots$ E2

Lee $\mathrm{K} \ldots \ldots \ldots \ldots \ldots$. . . . . . .

Lemus M ............G27

Lerer T . . . . . . . . . . . . . .C4

Lewis M ..............C34

Liley A . . . . . . . . . . . C37

Lim C . .............E2

$\operatorname{Lin} \mathrm{L} \ldots \ldots \ldots \ldots \ldots \ldots \ldots \ldots$

Lin $\mathrm{Y} \ldots \ldots \ldots \ldots \ldots \ldots$

Linares I ..............E34

Lindberg A $\ldots \ldots \ldots \ldots . . .67$

Linhares M . . . . . . . . . . B11

Liossi C . . . . . . 4. 202.2,4.304.3

Lisoway A . . . . .......... 3

Ljungman $G$. . . . 2.101.3,4.401.1,

.............4.401.3,F7,J12

Llewellyn N . . . . . . . . . . C37

Logan D . . . . . . . 4.403,4.403.1,

..............5.201,5.201.2

Lomba M ............E27

Lombart B . . . . . . G27,G28

Loughnan P ..........B9,B10

Lu Q . . . . . . . . . . . . . . 4.404.1

Luckett T . . . . . . . . . . . . B13

Lundeberg S ............. C7

Lundqvist $\mathrm{T} \ldots \ldots \ldots$. . . . B19

$\begin{array}{ll} & \mathbf{M} \\ \text { Macfarlane } G \quad \ldots \ldots \ldots \ldots \text { E12 }\end{array}$

Mahoney K ..............C14

Malin S . . . . . . . . . . G35

Malviya S . ..............D13

Manias E .............B9,B10

Manley J . . . . . . . . . . D16

Marche T .........E16,E17

Marti T ...........E27

Martin A ..............E9

Martinez F . . ............. 11

Martins Linhares M .... B20,B22

Maxwell L ... . . . . . . . . . C38

Mayes L . . . . . . . . 3.204,3.204.2

Mazaltarine G ......E23,G18

McCarthy AM ..2.504.1,2.504.2,

...............2.504.3,C11

McClain B ..........B18,C40

McCormick M .......E24,E26

McCracken LM …4.4.203.2,G5

McCue R ..............F3

McDougall C ............D15

McEown K ...........E17

McFadzean J ...........G37

McGrath P . . . . 2.203,A3,B15,

.B16,C30,D3,D4,

$\ldots \ldots \ldots \ldots$ D15,E9,E11,E31,G30

McMurtry CM ‥2.402.3,5.203.2

McMurtry M ..........G34

McNaughton K . . . . . B B1,B2,H4

Mehta M .......4.405,4.405.1,

........4.405.2,4.405.3,H2

Meier T ............H1

Meldrum M . . . . 4.205.1,4.205.3

Melin L . . . . . . . . . . . . .C17

Menezes C ............J18

Menke A

..E13
Miaskowski C ............................

Michels H .............C24

Middleton M ........B18,C40

Mincic A . . . ..............

Miró J . .........E19,G19

Miyase C . .............B11

Mogil JS .............2.601.3

Mohr K ..............G35

Mongia M ......4.405,4.405.1,

...........4.405.2,4.405.3

Montgomery K .........G10

Monti Fonseca L . . . . . . . . B22

Moon E ...............C30

Moraes Leite A … B20,B21,B22

Moreaux T . . . . . . . . . . C28

Morvan K ...........C28

Movahedi A .............C1

Mucha J ...............E29

Muir J . . . . . . . . . . .G23,J20

Munro J . . . ..........J6,J7

Murphy T ........C25,G16

Murray D ...........J17

Murray L . . ............ 222

Musclow S ............J20

Myers L . . . . . . . . . . . . C11

\section{N}

Nader R ........2.204.1,2.204.3

Nail L ...............F10

Narcy P . . . . . . . . G26,J8,J9

Nascimento L . . . . . . . . . . B20

Nelle M .............B12

Nelson K ...............B13

Nerge U . . . ............C45

Nielsen T ............J15

Nieto R ............G19,E19

Nikrooz L . . . . . . . . . . F1

Nilsson S .............G3

Niruthisard S ..........H5

Nkechi ............. 4.303.3

Noble L ... . . . . . . . . B8

Nogueira Góes F . . . . . . . . . . B22

Nolan A . . . ............C22

Norman E . . . . . . . . . . . . B23

Nouyrigat $V$. . . . . . G27,G28

Nugent A . . . . . . . .C12,C13

Nutkiewicz M . . . . 4.205.2,4.205.3

Nuyt A . . . . . ......B1,B2

\section{O}

Oberlander T . . . . . . . . . 2.404

O'Brien K . . . . . . . . . . . . . . B15

Oddson B . . . . . . . . D3,D4

Ofenstein J ................

Offenbächer M .........C24

Ohlsson A .....B8,B14,B15,B16

Ökland P . . . . . . . . . . B23

Olawuyi $\mathrm{O} \ldots \ldots \ldots \ldots \ldots$. . . . 1

Olive $\mathrm{G} \ldots \ldots \ldots \ldots$ G26,J8,J9

Olivé R .........................

Olsson G . . . . . . . . C17,C20,E10

Olsson GL . . . . . . . . . . . . 4.203.3

O'Neal P . . . . . . . . . . . 4.303.3,G17

Onishko C . . . . . . . . . . . D17

Oppedisano S . . . . . . . . . . C36

Oulton $\mathrm{K} \ldots \ldots \ldots \ldots$. ........ 36 


\section{Riddell RP .....2.204.3,2.501.1}

Palermo T . . . . . 2.205,2.205.2, $\ldots \ldots \ldots .4 .403 .4 .403 .4 .404 .3$, E15,G15 Palozzi L . . . . . . . . . . . J16,J17 Paredes S . . . . . . . . . E19,G19 Passchier J . . . . 5.201,5.201.1,E8 Pasturel A ..........C28,G31 Patterson $S \ldots \ldots \ldots \ldots . \ldots . \ldots 18$ Paul S ............................ 15 Pawar DK ......2.202.3,D6,D7 Payvandi M ........................... Pellico G . . . . . . . . B3,D5 Pellmann K ......................... 42 Penrose $S \ldots \ldots \ldots \ldots \ldots . . \ldots$ J6,J7 Perrusson $\mathrm{O} \ldots \ldots \ldots \ldots \ldots$. . . 28 Pescatello L .............C4 Petch E ..............J18 Peters J . . . A A1,A2,B17,E20,G29 Peters JBW ..........2.403.1, . . . . . . . . . . . . . . . . . . . . . . . .

Petrie J ..............................

Petrie-Thomas J ............B6 Petroz $G \ldots \ldots \ldots \ldots \ldots$. . . . . . 30 Pietilä A . . . . . . . . . E14 Piira T . . . 3.201,3.201.1,5.204, $.5 .204 .1,5.204 .2, \mathrm{~F} 5$

Pillai Riddell R .......E28,E33 Pirrallo R ...........J10 Pitts R ............G14

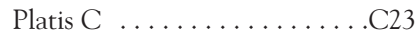
Poirot J . . . . . . . . . . . . . . C28 Polaner D .............G10 Pons P . . . . . . . . . . . C27

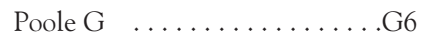
Pope H ..............J13 Popovski K ..........J16,J20 Porfyris S . . . . . . . . . . . C44 Potash L . . . . . . . . . . . . . D16

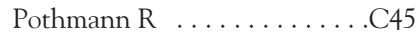
Prkachin $\mathrm{K} \ldots \ldots \ldots \ldots$ E36 Putnam J .............E15

\section{$\mathbf{R}$}

Rae J .................J20 Raffo M ..............E29 Raghavendran S . . . . . . . . G23 Ranger M .........C14,G23 Rasekh A .............C2 Rasekh H ................ 1

Reader S ............... 88 Reaney R ..............C35 Record E .............G14 Reed B ...............E26 Reed R ..............E24 Reiffer-Wiesel B ... . . . . . . . C19 Reinert P . . . . . . . . . . . . 26,J8,J9 Reiter F . . . . . . . .G21,G28 Ricard C . . . . . . .G27,G28

Ricard R . . . . . . . . . . C27

Richard H ............C39

Richards P ............. D13

Richter M ............C24
Ridling D . . . . . . . . . J2

Ridout D ............................

Rittgers $S \ldots . . \ldots \ldots \ldots$. . . E25

Rivadeneyra M .........E29

Rivard P . . . . . . . . . . . . . . . . . . .C12, C13

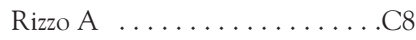

Robertson S ............G24

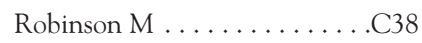

Rocha E ..............E36

Ronen I . . . . . . . . . C23,G25

Roofthooft D ..........G32

Ros A ................

Rose J . . . . . . C43,C38,D13,F3

Rosen B ..............F8

Rosén I . . . . . . . . . . . . . . . B23

Rosko E ..............F13

Rostami S . . . . . . . C1,C2,F1

Roth-Isigkeit A . . . . . . . . . H1

Roux $\mathrm{S} \ldots \ldots \ldots \ldots \ldots \ldots 28$

\section{S}

Sabzi Z ..............C2 Sadanand V . . . . . . . . . . . C44 Sadhu M ............H2 Sagar R . . . . . . . 4.405,4.405.1, . . . . . . . . . 4.405.2,4.405.3

Sambucco G ..........G7

Sankotara R ...........H3

Saroyan J .............G33

Sato A . . . . E4,E5,E21,E22,E30

Sauer C . . . . . . . . . . . C19

Savedra M .............................

Sawhney M ...........J20

Schanberg L ............E25

Schechter N .........C4,J5

Schechter W .........G33

Schlosser A ............C24

Schouw R .......A1,A2,E20

Schouw RHJA . . 4.305.1,4.305.2

Schrum S . . . . . . . . . C34

Schutte D .2.504.1,2.504.2,2.504.3

Schütz N ...........B12

Seers K .............G24

Segerberg M .............C7

Sehgal L . . . . . . . . . . . . . . D7

Seksarn P ...........H5

Seri L . . . . . . . . 3.204,3.204.3

Shah $\mathrm{P} \ldots \ldots \ldots \ldots \ldots$. . . . . B 8

Shah V .......B4,B8,B14,D16

Sharp M . . . . . . . . . . . . . C42

Shaw $\mathrm{H} \ldots \ldots \ldots \ldots$. . . . F 8 F4

Sherry D ............F3

Sidani $S \ldots \ldots \ldots \ldots \ldots 30$

Silman A .............E12

Silva M .............G2

Silvan Scochi C . . . B20,B21,B22

Silverman A ...........E22

Sim M ...........C41,G37

Simons L . . . . . . 3.204.3,E24,E26

Simonsen B ..........J15

Simpson D .............J10
Sgro M ...............D16

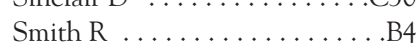

Sourbut C ........................

Spagrud L ..........E17,G9

Spamer M .............C24

Stallwood L ............G10

Stanford E ..............E18

Stangebye-Nielsen R ... . . . .C29

Stenekes ..............E6

Stevens B . . . 2.501.3,5.101.3,B14, . B15,B16,E28,E33,G30

Stinson J . . . 2.203,2.203.3,2.503,

$\ldots \ldots \ldots \ldots$. . . . . . . . . . . . . . . .

Stoffel L . . . . . . . . . . . B12

Storm V .............J15

Streiner D . . . . . . . . . G30

Stromback C ...........E10

Subramaniam R ........... D6

Sunder R ...............H6

Suresh S . . . . . . . . . . . C44

Sutters K .............C15

Suwanraj M ..........G20

Symons F . . . . . . . . C12,C13

Szewczuga D ............J10

\section{T}

Taddio A . . . . . . 2.404,6.101.1, $\ldots \ldots \ldots \ldots$ B4,B8,B14,D16

Tassone R . . . . . . . . . . C5

Taylor A ...............J13

Teisseyre L . . . . . . . . . . C28

Thibault $\mathrm{P} \ldots \ldots \ldots \ldots \ldots$. E29

Thienthong $S \ldots \ldots \ldots$.......H3

Thomas J . . . . . . . 5.202,5.202.3

Thompson A ...........624

Thompson J ............G35

Thomson N ...........J20

Tibboel D . . . . . . . 2.401,4.305.1, . . . . . 4.305.2,5.202.2,A1, A2,E20,F12,G29,G32

TiemeierH $\ldots \ldots \ldots \ldots \ldots \ldots$ E8

Tobias J . . . . . . . . C21,D11,D12

Tonnelli A . . .........E23

Tourniaire B .....D14,E23,G18, . . . . . . . G21,G27,G28

Tsao J . . . . 4.205.1,4.205.2,4.205.3

Tu M ..............

Turquin $\mathrm{P} \ldots \ldots \ldots$ G27,G28

Tutag Lehr V ............. B5

Twycross A ...........J3

Tyrrell J ..........J18

$\mathbf{U}$
$\mathrm{Uman} L$
$\mathrm{H}$

Valois $\mathrm{T} \ldots \ldots \ldots \ldots \ldots$ G 23

van Blijderveen $G \quad \ldots \ldots \ldots . G 32$

Van den Anker J . . . 2.401,2.401.2

van den Berg $N \ldots \ldots \ldots$. E8

van Deventer P . . . . . . . . .G32

van Dijk M . . . . . 5. 5.202,5.202.2,

.F12,G32 an Lingen R . . .4.301,4.301.1,B12

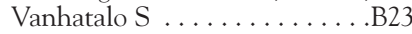

Veerkamp J .............G8

Vehviläinen-Julkunen K .....E14

Verhulst F ..........E8

Versloot J .............G8

Vervoort T . . . . 2.204.2,2.204.3

Vieyra M ......................... 31

Villeneuve E ...........C18

Visram ............E7

von Baeyer C . . . . 2.203,2.204.3, 2.403.1,2.505,2.505.3, .E7,E16,G9,G12,G34,J19

von Essen $\mathrm{L} \ldots \ldots \ldots \ldots$. . . . F7

\section{W}

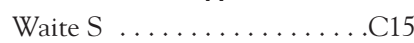

Walco $G \ldots \ldots \ldots \ldots \ldots 22$

Walker C .............B1,B2

Walker L . . 2.101.2,3.204,3.204.1

Wamsler C ............E13

Washington L .............E3

Watson L .............J13

Watt-Watson J ............J20

Webber K ............J20

Weidner N ............4.302

Weinberg J ...............B6

Weinberg M .... 3.204,3.204.2

Weisman S . . . 3.203,4.201,C42,

$\ldots \ldots \ldots \ldots$ E4,E5,E21,E22,E30

Wells M ...........G33

Wester I . . . . . . . . . . . . . C29

Whitaker BH ........ 4.202.3

Whitehead P ...........C3

Whitfield M .............B6

Wicksell RK ...4.203.3,C17,C20

Williams M .......................... 26

Williams S . . . . . . 3.204,3.204.1

Williams T .............. 3

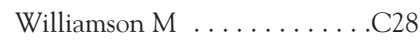

Wilson $\mathrm{S} \ldots \ldots \ldots \ldots \ldots \ldots$. E3

Winsö E .................. 229

Wolfe J ............. 4.204.1

Wolff ............E8

Wood C . . . . . . 4.304.1,C28,G31

Wootton J ..............E25

WuE . . . . . . . . . 4.404.2

\section{$\mathbf{Y}$}

Yimyaem $\mathrm{P} \ldots \ldots \ldots \ldots \ldots \mathrm{H}^{3}$

\section{Z}

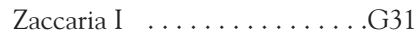
Zegarra J ............E29 Zeltzer L . . . . 4.205.1,4.205.2,G33 Zempsky WT . . . 2.502,C4,D13,J5

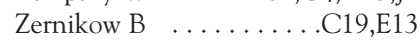

Zeskind $\mathrm{P} \ldots \ldots \ldots \ldots \ldots \ldots$ B5

Zimmermann L .........B12

Zisk RY . . . . . 4.303.2,E14,F6,G13

Zohsel K . . . . . . . . . . D9,D10

Zuk J ..............G10

Zurakowski D ...........C5 


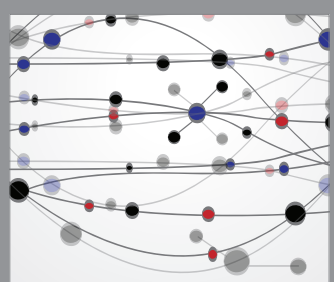

The Scientific World Journal
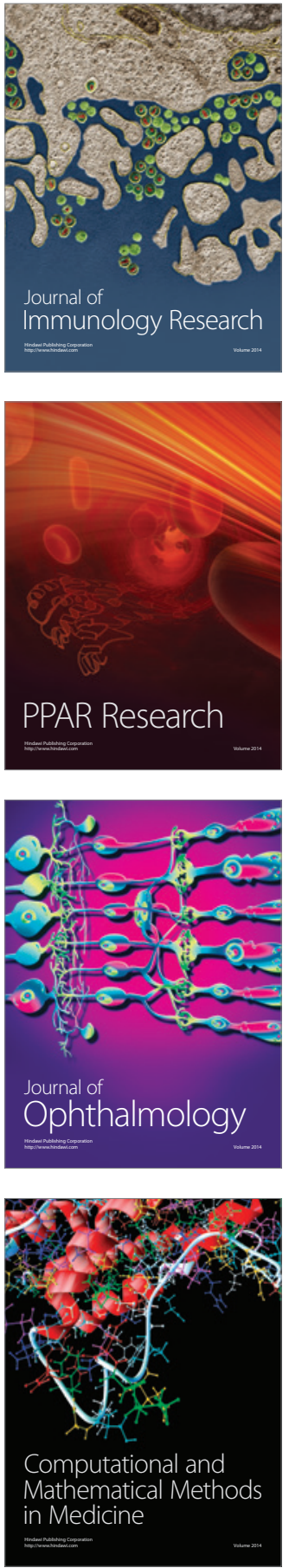

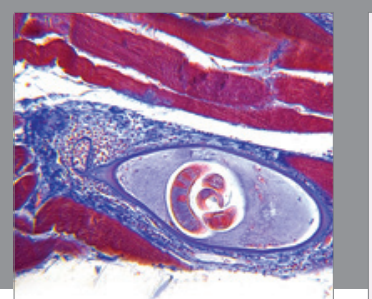

Gastroenterology Research and Practice

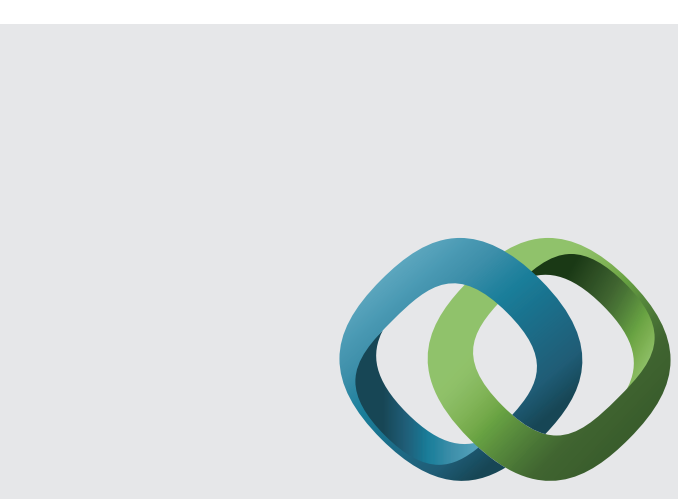

\section{Hindawi}

Submit your manuscripts at

http://www.hindawi.com
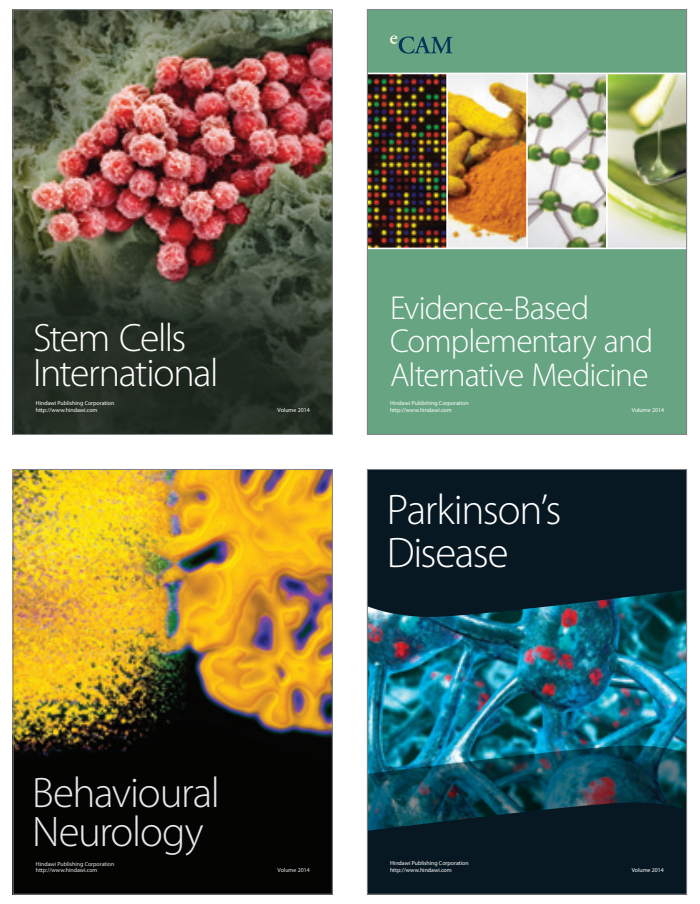
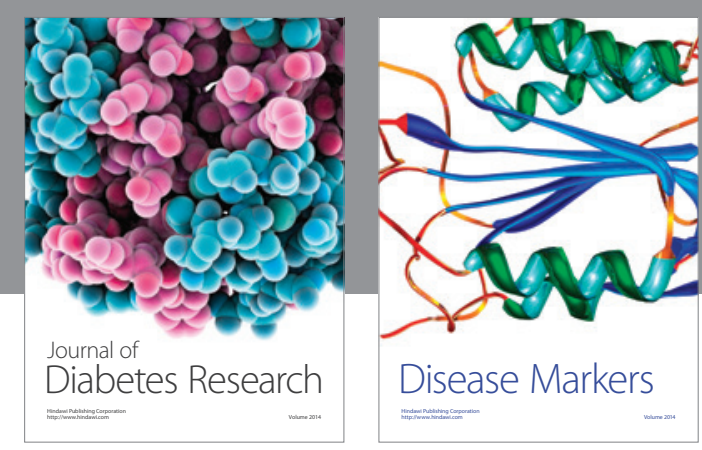

Disease Markers
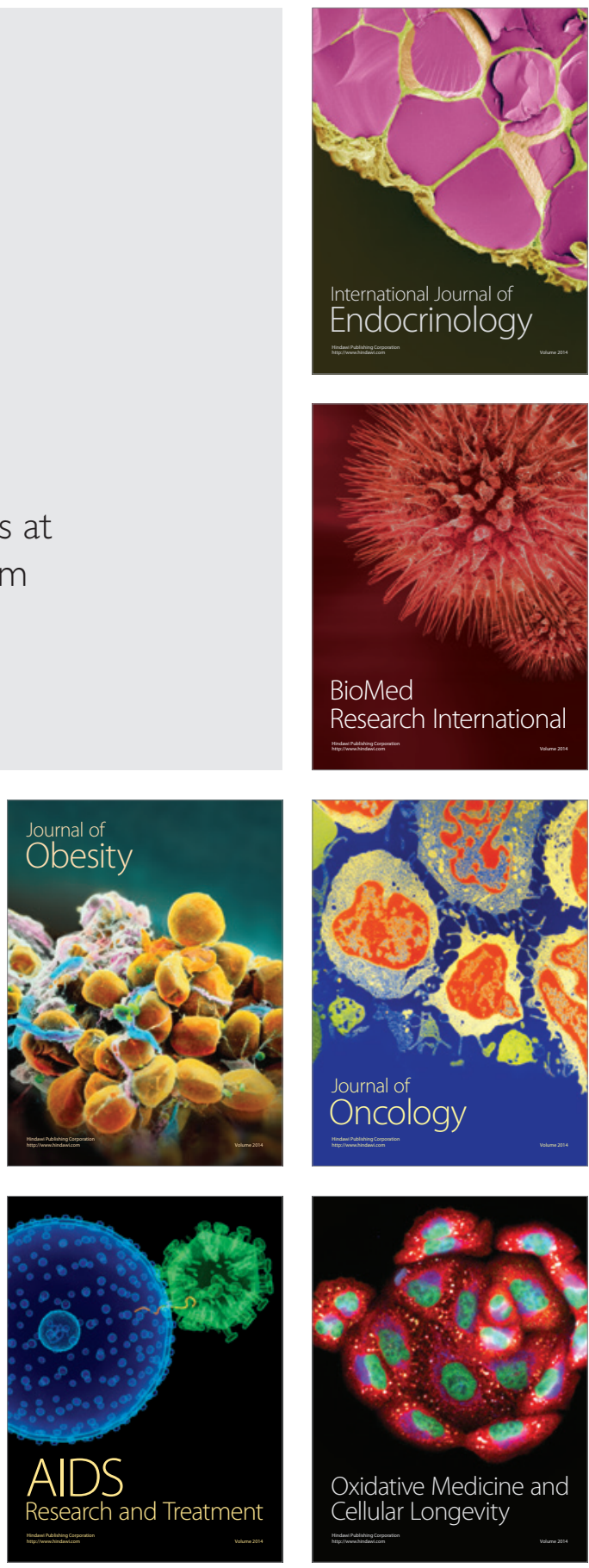